\title{
PRESSURE NORMALIZATION OF PRODUCTION RATES IMPROVES FORECASTING
}

\section{RESULTS}

\author{
A Thesis
}

by

JUAN MANUEL LACAYO ORTIZ

\author{
Submitted to the Office of Graduate Studies of \\ Texas A\&M University \\ in partial fulfillment of the requirements for the degree of \\ MASTER OF SCIENCE
}

$\begin{array}{ll}\begin{array}{l}\text { Chair of Committee, } \\ \text { Committee Members, }\end{array} & \begin{array}{l}\text { Dohn Lee } \\ \text { Maria Barrufet }\end{array} \\ \text { Head of Department, } & \text { Dan Hill }\end{array}$

August 2013

Major Subject: Petroleum Engineering

Copyright 2013 Juan Manuel Lacayo Ortiz 


\begin{abstract}
New decline curve models have been developed to overcome the boundary-dominated flow assumption of the basic Arps' models, which restricts their application in ultra-low permeability reservoirs exhibiting long-duration transient flow regimes. However, these new decline curve analysis (DCA) methods are still based only on production rate data, relying on the assumption of stable flowing pressure. Since this stabilized state is not reached rapidly in most cases, the applicability of these methods and the reliability of their solutions may be compromised. In addition, production performance predictions cannot be disassociated from the existing operation constraints under which production history was developed. On the other hand, DCA is often carried out without a proper identification of flow regimes. The arbitrary application of DCA models regardless of existing flow regimes may produce unrealistic production forecasts, because these models have been designed assuming specific flow regimes.
\end{abstract}

The main purpose of this study was to evaluate the possible benefits provided by including flowing pressures in production decline analysis. As a result, it have been demonstrated that decline curve analysis based on pressure-normalized rates can be used as a reliable production forecasting technique suited to interpret unconventional wells in specific situations such as unstable operating conditions, limited availability of production data (short production history) and high-pressure, rate-restricted wells. In addition, pressure-normalized DCA techniques proved to have the special ability of dissociating the estimation of future production performance from the existing operation constraints under which production history was developed. On the other hand, it was also observed than more consistent and representative flow regime interpretations may be obtained as diagnostic plots are improved by including MBT, pseudovariables (for gas wells) and pressure-normalized rates. This means that misinterpretations may occur if diagnostic plots are not applied correctly. 
In general, an improved forecasting ability implies greater accuracy in the production performance forecasts and more reliable reserve estimations. The petroleum industry may become more confident in reserves estimates, which are the basis for the design of development plans, investment decisions, and valuation of companies' assets. 


\section{DEDICATION}

From the depth of my heart I desire to dedicate this work to my darling princess Valeria, who is the energy of my soul, my proud, and my greatest inspiration.

To my beloved wife Monica. The brightness of her eyes, her sweet smile, and her never-ending love make my life happier than I ever imagined.

To my parents, grandparents and siblings. The effort and the sacrifice of this work express the perseverance, the strength, and all the wonderful values of our family.

Finally, to my country Colombia, because I trust our peace is possible. 


\section{ACKNOWLEDGEMENTS}

First of all, I praise and thank God for blessing me with this opportunity.

I would like to express my deepest appreciation to my Sensei and committee chair, Dr. Lee, who was kind enough to guide me with dedication and wisdom throughout the course of this research. I would like to thank my committee members, Dr. McVay and Dr. Barrufet, for their significant advice and recommendations. Thanks also go to my research fellows, to the PDCA application developers, especially to Raul Gonzalez, to the Writing Center, and to my editors-in-chief Caitlin and Nef.

I also want to extend my gratitude to my sponsor ECOPETROL, particularly to the VicePresidency of Exploration and those who gave me their support. My special gratitude goes to Javier Sanchez, who believed in me and encouraged me to pursue this dream. He is responsible for making this wonderful adventure possible.

Thanks to my wife Monica and my daughter Valeria for their unconditional support, patience, and love. I will always remember the great moments that I have spent with my family and friends in College Station. Thanks y'all for making this Master's program an awesome experience of life. My family and I will miss you and will be waiting for you in Colombia. We hope to come back some day to have more tailgates.

Finally, thanks to Texas A\&M University, to College Station, and to Texas. I have the greatest admiration for these friendly people, their amazing culture, and their valuable traditions.

Gig'em Aggies...!!! 


\section{NOMENCLATURE}

\section{Abbreviations}

a Duong's intercept constant or LGM constant

B Formation volumetric factor, RB/STB

b Hyperbolic exponent

$b^{\prime} \quad y$-intercept of the square root-time plot

$C_{t} \quad$ Total compressibility, $\mathrm{psi}^{-1}$

$D \quad$ Decline rate or production loss ratio, $D^{-1}$

$D_{i} \quad$ Initial decline rate, $\mathrm{D}^{-1}$

$D_{\infty} \quad$ Decline constant at "infinite time", $\mathrm{D}^{-1}$

$\widehat{D}_{i} \quad$ Decline constant, $\mathrm{D}^{-1}$

$F_{c d} \quad$ Fracture conductivity, md-ft

$G_{p} \quad$ Cumulative gas production, SCF or MMSCF

$h \quad$ Reservoir net pay, $\mathrm{ft}$ 
J Productivity index, MSCF/D/psi

K $\quad$ Carrying capacity

K Permeability, md

$k_{f} \quad$ Fracture permeability, md

$M_{i} \quad$ Output of flow regime diagnostic plots

$m \quad$ Slope of a straight line (Duong, linear, and bilinear plots), unit/log cycle

$n \quad$ Time or hyperbolic "exponent"

$P E \quad$ Percent error, \%

$P(F R) \quad$ Probability of flow regime occurrence, \%

$p \quad$ Pressure, $\mathrm{psi}$

$p^{*} \quad$ Average static reservoir pressure, psi

$p_{i} \quad$ Initial pressure, psi

$p_{w f} \quad$ Flowing pressure, psi

$q$ Production rate at time $\mathrm{t}, \mathrm{MSCF} / \mathrm{D}$ or STB/D

$q_{\text {corr }} \quad$ Corrected production rate, MSCF/D or STB/D 


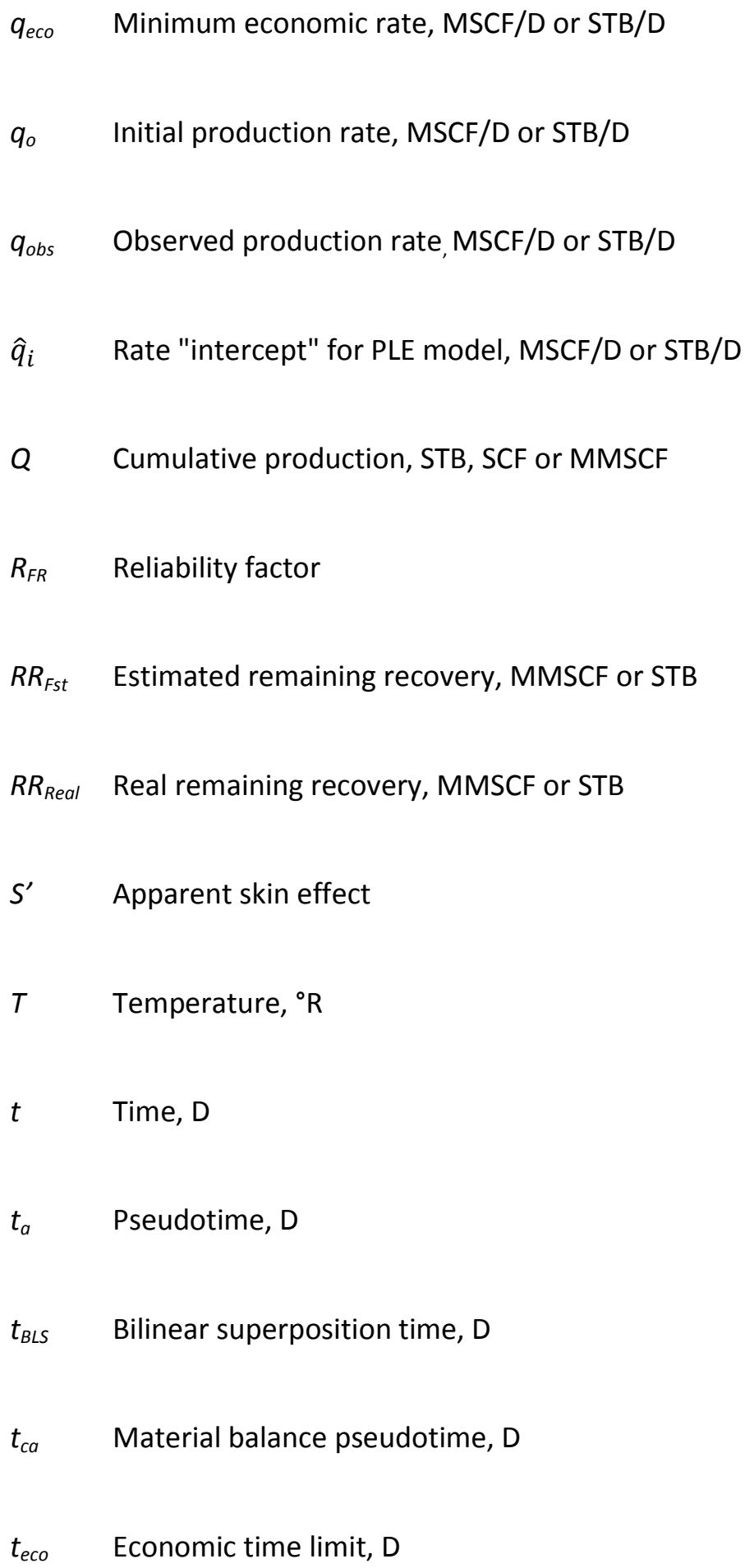




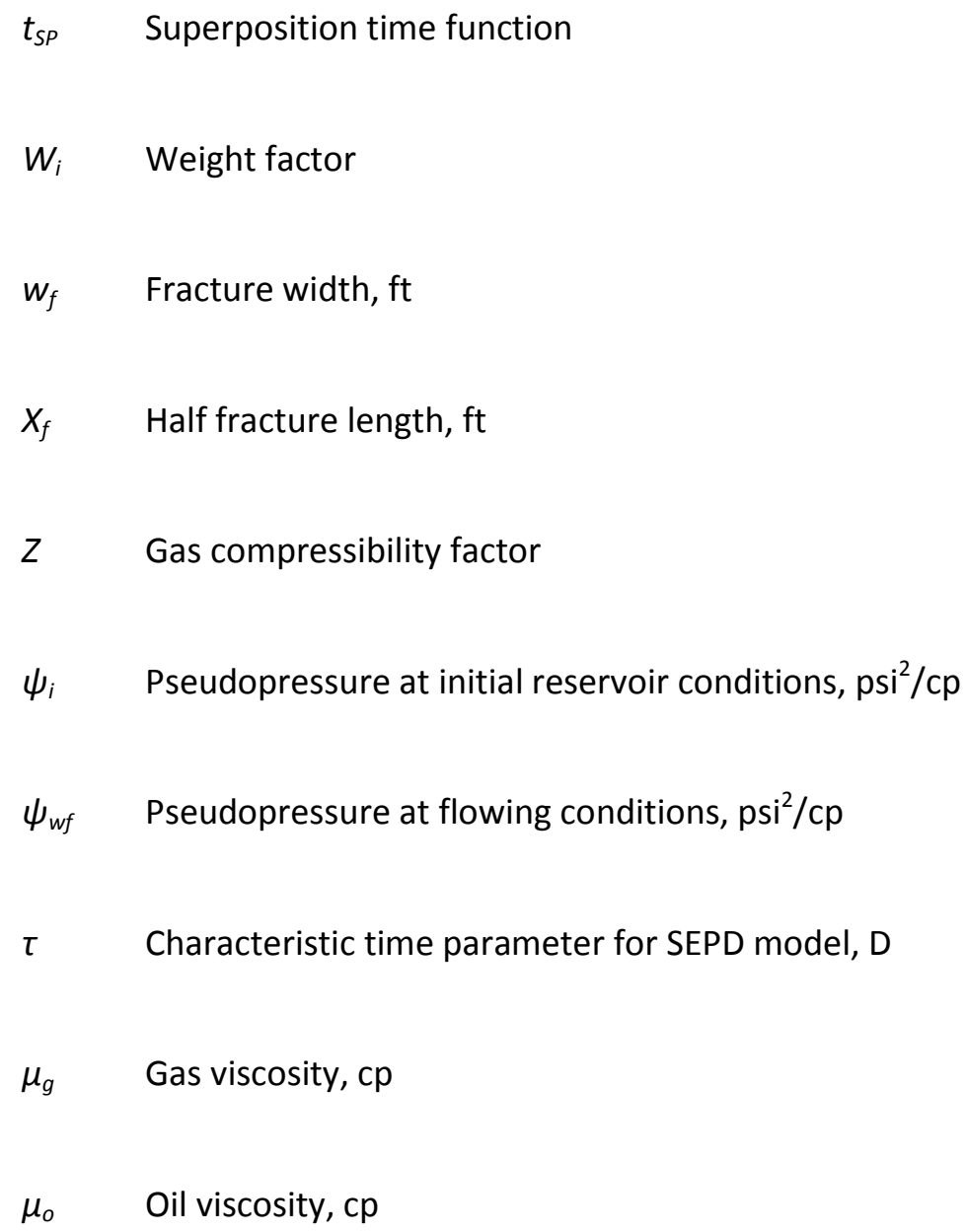

\section{Acronyms}

$\begin{array}{ll}\text { BDF } & \text { Boundary-Dominated Flow } \\ \text { DCA } & \text { Decline Curve Analysis } \\ \text { EUR } & \text { Expected Ultimate Recovery }\end{array}$


Power Law Exponential Method

PN

Pressure Normalization

PN2

Square Pressure Normalization Method (SPN)

PNR

Pressure-Normalized Rates

PN-DCA Pressure-Normalized Decline Curve Analysis

PTA Pressure Transient Analysis

RNP Rate-Normalized Pressures

RTA Rate Transient Analysis

SEPD Stretched Exponential Production Decline Model

SNR

Square Pressure Normalized Rates

SPN

Square Pressure Normalization (PN2) 
Stimulated Reservoir Volume

$\Delta \mathrm{PF}$

Pressure Correction Factor $\left(q_{\text {corr }}\right)$

$\Delta \Psi N$

Pseudopressure Normalization method $(\Psi N)$

$\Psi N$

Pseudopressure Normalization $(\Delta \Psi N)$

$\Psi N R$

Pseudopressure-Normalized Rates 


\section{TABLE OF CONTENTS}

ABSTRACT

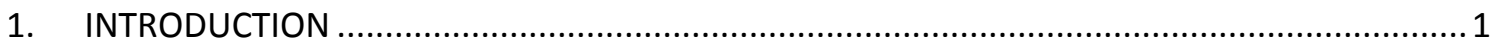

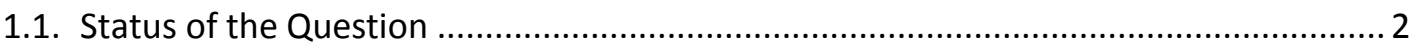

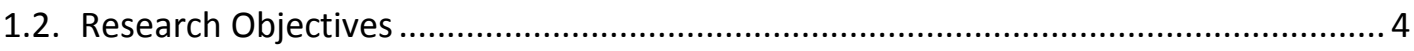

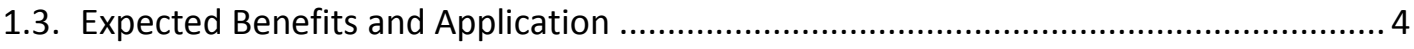

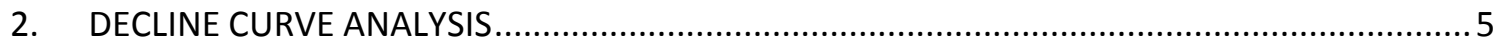

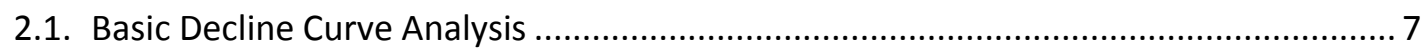

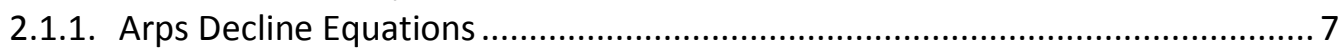

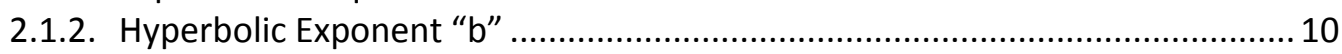

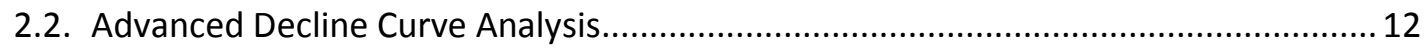

2.2.1. Application Of Decline Curve Analysis To Unconventional Reservoir .............. 12

2.2.2. Stretched Exponential Production Decline Model (SEPD) .............................. 14

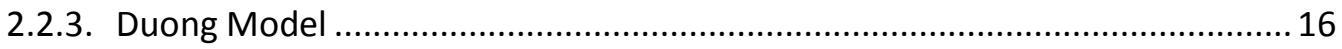

2.2.4. Power Law Exponential Method...................................................................... 19

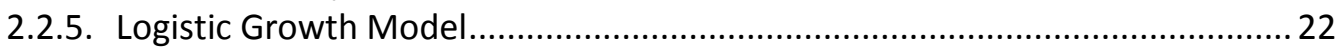

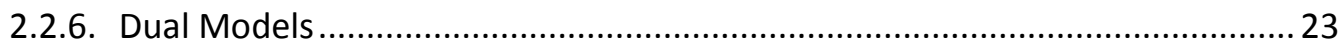

3. RATE TRANSIENT ANALYSIS APPLIED TO MULTI-FRACTURED HORIZONTAL WELLS IN

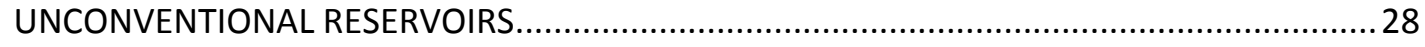

3.1. Superposition Time Functions and Gas Flow Considerations ................................... 30

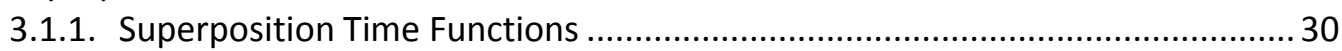

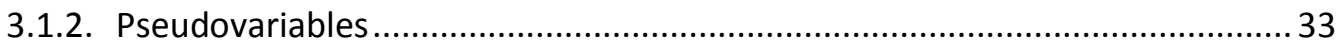

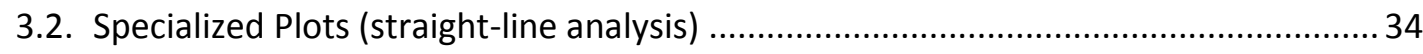

3.2.1. Flow Regimes in Multi-Fractured Horizontal Wells ........................................ 34

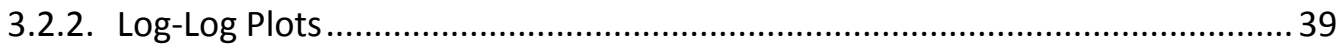




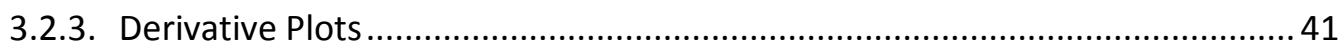

3.2.4. Fourth Root-Time Plot (Bilinear Flow) ......................................................... 42

3.2.5. Square Root-Time Plot (Linear Flow) ........................................................ 43

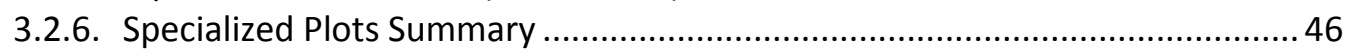

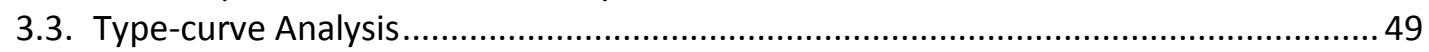

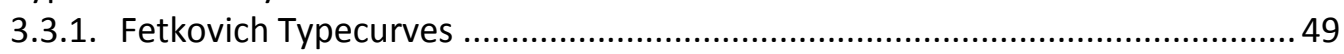

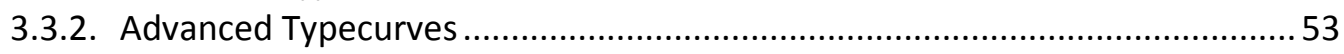

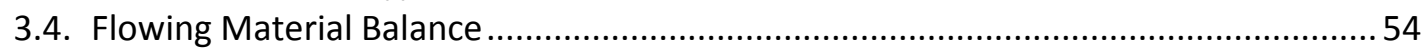

3.5. Analytical Models (Multi-Fractured Horizontal Wells).............................................. 55

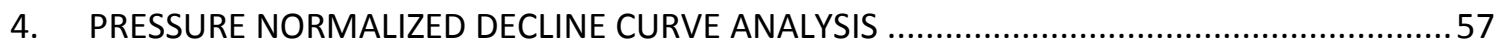

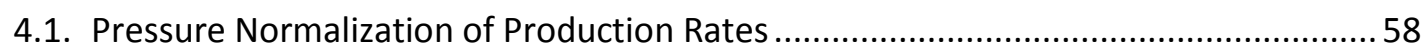

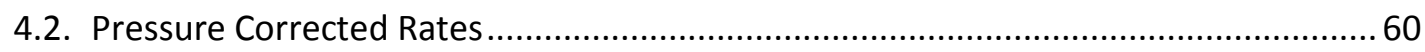

4.3. Applicability of Pressure-Normalized Decline Curve Analysis.....................................61

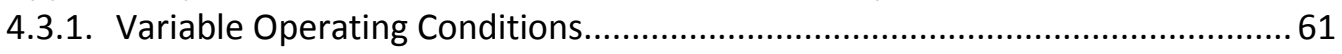

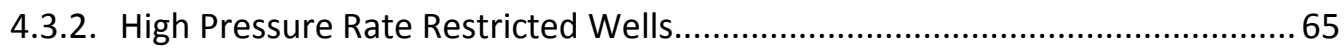

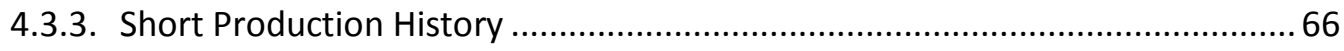

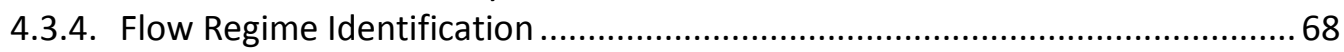

4.4. Production Forecasting Techniques For Pressure-Normalized Rates ..........................68

4.4.1. Decline Curve Analysis Based on Normalized Rates ..........................................68

4.4.2. Harmonic Decline of PNR vs. Cumulative Production .................................... 70

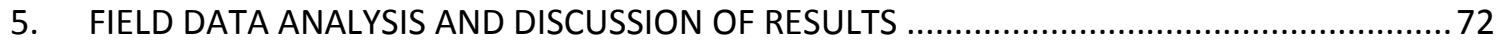

5.1. Description of the Analysis Workflow (Methodology) .............................................. 72

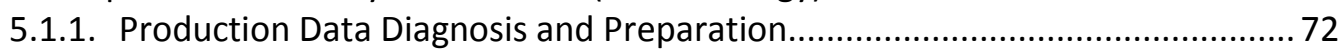

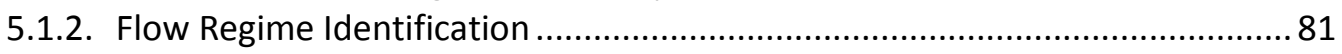

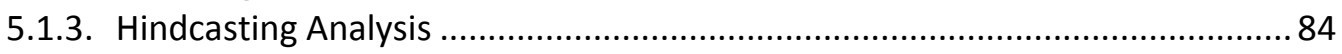

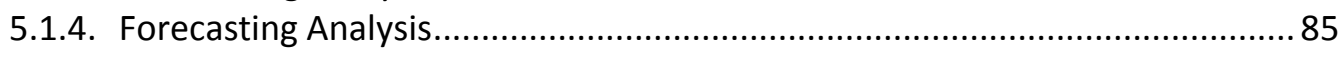

5.2. Evaluation of Pressure-Normalized Decline Curve Analysis (PN-DCA) as

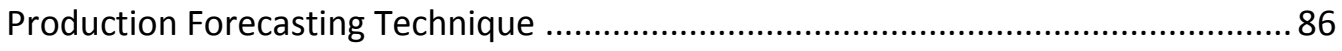

5.2.1. Production Data Diagnosis and Preparation................................................. 86

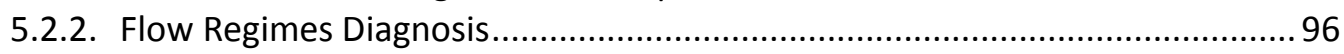

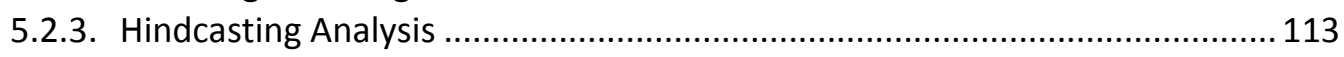

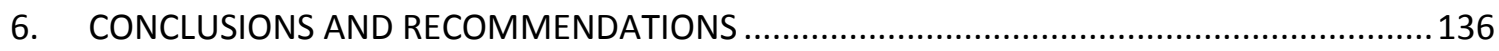

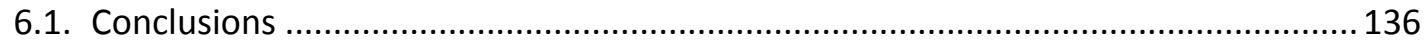

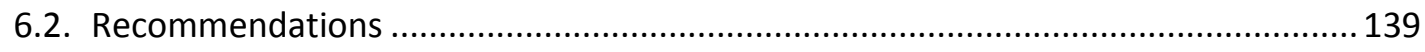

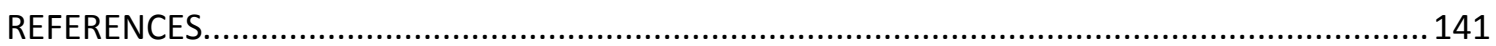




\section{LIST OF FIGURES}

Fig. 1- Traditional Arps Decline Curves (Fekete, 2013a)...................................................... 10

Fig. 2- Decline exponents " $b$ " for various reservoir drive mechanisms (Fetkovich, 1996).

Fig. 3- q/Gp vs t plot for varios shale gas wells (Duong, 2010). ...........................................18

Fig. 4- Schematic plot for hyperbolic and power law exponential models (Ilk et al., 2008)

Fig. 5- Production data analysis by applying LGM (Clark et al., 2011) ...................................23

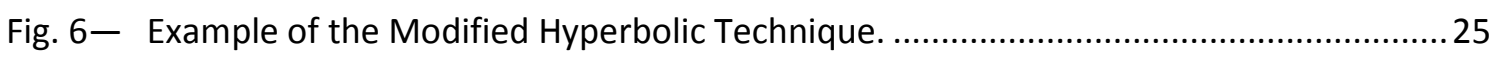

Fig. 7- Comparison of original Duong and modified Duong models (Joshi and Lee, 2013)

Fig. 8- Material Balance Time plot showing the equivalency of qD and 1/pD (Fekete, 2013a).

Fig. 9- Illustration of the Material Balance Time concept (Fekete, 2013a) .....

Fig. 10-Example of flow regimes in a MFHW generated by streamlines simulation (Luo et al., 2010) and flow regime signatures in a radial derivative plot (Clarkson, 2013a)

Fig. 11-Example of flow regimes identified by using a Log-Log plot.

Fig. 12-Illustration of flow regime sequence for a MFHW in a single porosity reservoir (Clarkson and Pedersen, 2010).

Fig. 13-Example of the squared root time plot (Clarkson, 2013a)... 45

Fig. 14-Example of straight-line specialized plots (a: linear, b: elliptical, c: radial, d: BDF).

Fig. 15-Identification of flow regimes by applying different specialized plots (a: LogLog, b: Transient productivity index, c: Radial derivative, d: Linear derivative) by Clarkson (2013b).

Fig. 16-Fetkovich "transient" rate type curve (Fetkovich, 1973) .............................................50

Fig. 17-Fetkovich "empirical" rate type curve (Fetkovich, 1973).

Fig. 18-Fetkovich typecurves: Composite of analytical and empirical solutions with comments (After Fetkovich, 1980). 
Fig. 19-Fetkovich typecurves: Composite of analytical and empirical solutions including the linear solution (Chen and Teufel, 2000)

Fig. 20-Blasingame integral-derivative for fractured horizontal wells (Fekete, 2013a). ...........53

Fig. 21-Illustration of the concept of rate integral (Fekete, 2013a)..........................................54

Fig. 22-Application of the dynamic material balance technique to a shale gas well.................55

Fig. 23-Application of pressure corrected rates in diagnostic plots to compensate for the early choke-back effect (Duong, 2010). .................................................................6 60

Fig. 24-Plot of Pwf vs. time for a high pressure rate restricted shale well. ...............................61

Fig. 25-Example of the effect of changing operating conditions on production rates..............63

Fig. 26-Example of an unconventional shale gas well producing under variable

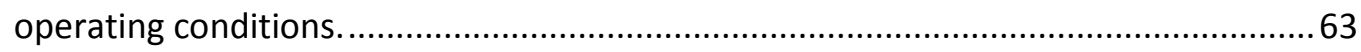

Fig. 27-Example of monthly averaged and filtered production rates......................................64

Fig. 28-Example of a high pressure, rate restricted gas well (EF-4) ......................................... 65

Fig. 29-Example of unstable flowing conditions at early production times..............................67

Fig. 30-Illustration of the application of decline curve analysis using pressurenormalized rates. Red and green lines represent PN-DCA method, while blue lines correspond to the traditional application of DCA models.

Fig. 31-Pressure normalized rate versus cumulative production. ........................................... 71

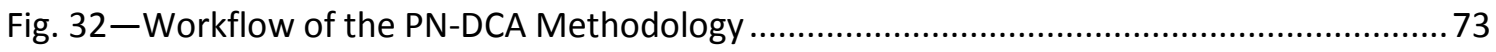

Fig. 33-Correction of production rates from a shale gas well................................................. 80

Fig. 34-Location of unconventional wells used in this study. After Gale and Holder (2010). 87

Fig. 35-Histogram of the production histories duration for all wells...................................... 88

Fig. 36-Production history times (minimum, mean and maximum) for each play...................90

Fig. 37-Production rates and flowing pressures of $\mathrm{M}-1$ well. ................................................ 91

Fig. 38-Production rates of well M-1 presented in daily and monthly format .........................92

Fig. 39-Liquid loading diagnostic plot (Turner plot) of well M-1...........................................93

Fig. 40-Histogram of initial reservoir pressures for each play. .................................................93

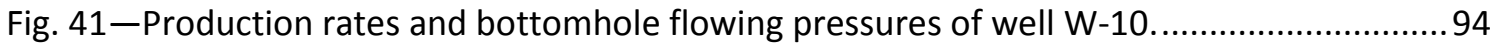

Fig. 42-Production rates and flowing pressures of well EF-7 ...............................................95 
Fig. 43-High pressure, rate controlled Eagle Ford well (EF-2) ............................................... 95

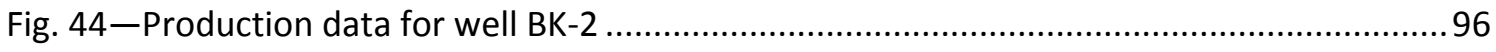

Fig. 45-Comparison of bilinear diagnostic plots for gas wells according to the single method approach. 100

Fig. 46-Comparison of bilinear diagnostic plots for oil wells according to the single method approach. 100

Fig. 47-Bilinear flow diagnosis according to the multi-method approach. 102

Fig. 48-Identification of linear flow regime for well EF-2. Top: Log q - Log t; middle: Log q - Log MBT; bottom: Log $q / \triangle P$ - Log MBT. Dash line represents linear flow (slope: $-1 / 2$ ).

Fig. 49-Diagram showing the occurrence of side effect when using pressurenormalized rates in Eagle Ford cases.

Fig. 50-Transition signature becomes dominant and linear signature shortens when pressure-normalized rates are used. Dash line: linear flow, dotted line: bilinear flow, solid line: BDF.

Fig. 51-Comparison of BDF diagnostic plots for gas wells according to the single method approach.

Fig. 52-Comparison of BDF diagnostic plots for oil wells according to the single method approach.

Fig. 53-BDF diagnosis according to the multi-method approach......................................... 108

Fig. 54-Statistics of BDF onsets for each play (minimum, mean and maximum). 109

Fig. 55-FRI analysis of well M-1. Dash line: linear flow, dotted line: bilinear flow, solid line: BDF. From left to right and from top to bottom: $\log q-\log t, \log q-\log$ $M B T$, Log $q / \Delta P$ - Log MBT, Log $q / \Delta \Psi$ - Log Tca, $\Delta P / q-S R(M B T), \Delta \Psi / q-$ $\mathrm{SR}(\mathrm{Tca}), \Delta \mathrm{P} / \mathrm{q}-\mathrm{FR}(\mathrm{MBT}), \Delta \Psi / \mathrm{q}-\mathrm{FR}(\mathrm{Tca})$.

Fig. 56-FRI analysis of well WF-1. Dash line: linear flow, dotted line: bilinear flow, solid line: BDF. From left to right and from top to bottom: $\log q-\log t, \log q-\log$ MBT, Log $q / \Delta P$ - Log MBT, Log $q / \Delta \Psi$ - Log Tca, $\Delta P / q-S R(M B T), \Delta \Psi / q-$ $\mathrm{SR}(\mathrm{Tca}), \Delta \mathrm{P} / \mathrm{q}-\mathrm{FR}(\mathrm{MBT}), \Delta \Psi / \mathrm{q}-\mathrm{FR}(\mathrm{Tca})$.....

Fig. 57-FRI analysis of well BK-3. Dash line: linear flow, dotted line: bilinear flow, solid line: BDF. From left to right and from top to bottom: $\log q-\log t, \log q-\log$ $M B T$, Log $q / \Delta P$ - Log MBT, Log $q / \Delta \Psi$ - Log Tca, $\Delta P / q-S R(M B T), \Delta \Psi / q-$ $\mathrm{SR}(\mathrm{Tca}), \Delta \mathrm{P} / \mathrm{q}-\mathrm{FR}(\mathrm{MBT}), \Delta \Psi / \mathrm{q}-\mathrm{FR}(\mathrm{Tca})$. 
Fig. 58-FRI analysis of well EF-3. Dash line: linear flow, dotted line: bilinear flow, solid line: BDF. From left to right and from top to bottom: Production rates and flowing pressures (THP), Log $q-\log t$, Log $q-\log M B T$, Log $q / \triangle P$ - Log MBT, $\Delta P / q-S R(M B T), \Delta P / q-F R(M B T)$.

Fig. 59-Example of production data preparation for a hindcasting analysis. Green points indicate the portion of real data that is used to match the DCA models. Remaining red points correspond to the production data that will be compare with the predicted decline curve.

Fig. 60-Hindcasting analysis of DCA models for well M-8. Red points indicate the portion of production history used to match the DCA models. Green points are the portion of the production history used to compare the accuracy of the forecasts provided by the DCA models. The red line represents the prediction start point (PSP).

Fig. 61-Application of hindcasting with PN-DCA for well M-8. ...........................................117

Fig. 62-Results of the comparative analysis, all wells included.

Fig. 63-Histogram of results (percent error) for each DCA technique in the general scenario (all wells included). The red line on each plot corresponds to the probabilistic function that best fits the results. This probabilistic function is presented only for visualization purposes, because comparative analysis is based on calculated data.

Fig. 64-Comparison of probabilistic functions representing the average tendency of the results for the general scenario (all wells included).

Fig. 65-Comparison of results for all DCA models (All wells included). ................................. 122

Fig. $66-$ Results of the comparative analysis, only gas wells included.................................... 124

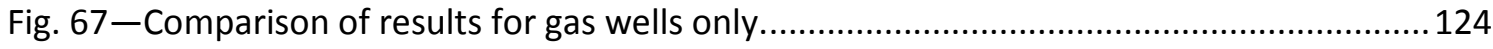

Fig. $68-$ Results of the comparative analysis, only oil wells included. ...................................126

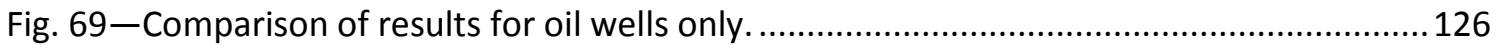

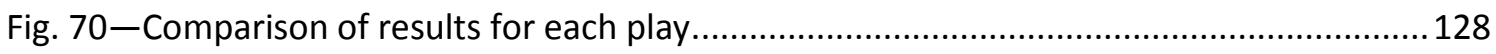

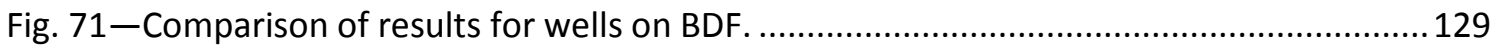

Fig. 72-Average prediction start times for each play......................................................... 132

Fig. 73-Forecasting improvement indexes for gas wells (Comparison between PSP 1 and 3) 
Fig. 74-Application of pressure-normalized rates in well EF-10. Data is presented in a daily basis format.

Fig. 75-Application of pressure normalizes rates in well EF-7. Data is presented in a

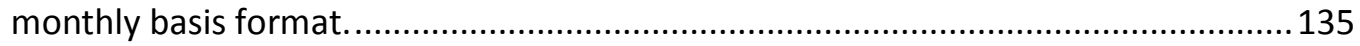

Fig. 76-Number of high pressure, rate controlled Eagle Ford wells identified in this study. 135 


\section{LIST OF TABLES}

Page

Table 1- Decline exponents "b" for various reservoir mechanisms (Fekete, 2013b)............... 11

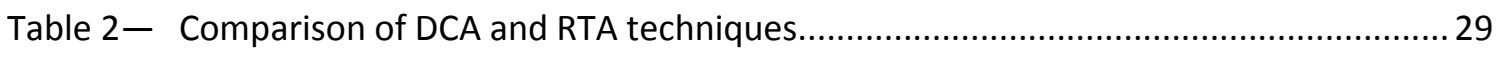

Table 3- Superposition time functions for different flow regimes (Agnia et al., 2012). .......... 31

Table 4- Log-Log plots for flow regime identification. ........................................................... 40

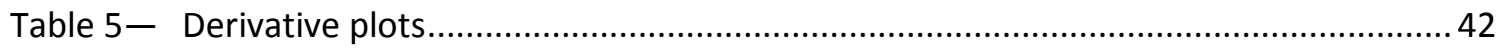

Table 6- Summary of specialized plots for straight-line analysis (Clarkson and

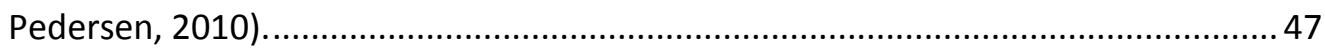

Table 7- Summary of pressure normalization methods and pressure-normalized rates

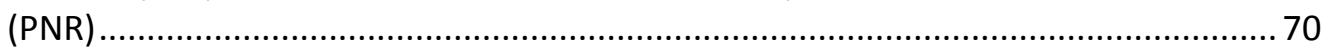

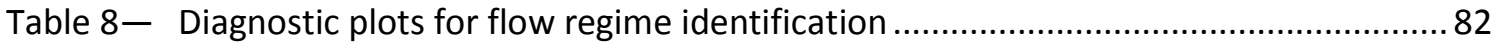

Table 9- List of wells used for the hindcasting analysis....................................................... 89

Table 10-Summary table, including flow regime identification results. ................................97

Table 11-Comparison of FRI results for gas and oil wells. ................................................... 99

Table 12-Bilinear flow diagnosis according to the multi-method approach............................. 101

Table 13-Weight factors for the multi-method approach. .................................................... 102

Table 14-BDF diagnosis by the multi-method approach.......................................................... 108

Table 15-Wells summary table, including prediction start points and BDF onsets. ................ 115

Table 16-Results of the comparative analysis of DCA techniques (general, oil, and gas

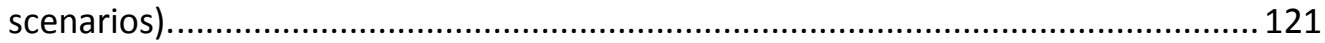

Table 17-Summary of results (mean percent error) for the general comparative analysis (all wells included).

Table 18-Summary of results (mean percent error) of the comparative analysis for gas wells.

Table 19-Summary of results (mean percent error) of the comparative analysis for oil

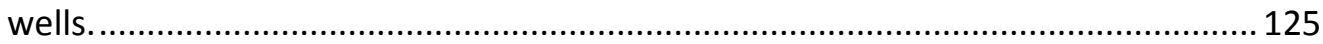

Table 20-Summary of results (mean percent error) for each play. ........................................ 127

Table 21-Summary of results (mean percent error) for wells exhibiting BDF. 


\section{INTRODUCTION}

Decline curve analysis is an empirical technique based only on historical production rate trends to predict future production performance. Thus, it is fundamentally considered to lack a basis in physical principles governing the fluid flow phenomena in the reservoir. In addition, Arps' decline models, also known as basic or traditional DCA, are grounded in the concept that boundary-dominated flow is the dominant flow regime and that operating conditions and reservoir properties remain constant. Therefore, decline curve analysis assumes that reservoir boundaries have been reached and that the main factors involved in the production decline process will remain constant during the future well life. In general, DCA techniques are extremely valuable tools for production analysis because of their simplicity. However, some of those features that make them simple also represent a disadvantage in other aspects, as presented below. As a consequence, erroneous reserve estimations may be obtained if DCA is applied without considering these limitations. According to Mattar and Anderson (2003), traditional decline curve analysis provides reasonable answers in many situations, but has its limitations, because it completely ignores flowing pressure data. Another important restriction of decline curve analysis is its inability to disassociate the estimation of future production performance from the existing operation constraints under which production history was developed (Mattar and Anderson, 2003).

In recent years, as a result of the increasing exploration and production activity in unconventional plays, new decline curve models (also known as advanced decline curve models) have been developed. The main purpose of these new models-e.g., SEPD, Duong, Logistic Growth, Power Law, Dual Models-is to overcome the boundary-dominated flow assumption of the basic Arps' models, which restricts their application in ultra-low permeability reservoirs exhibiting a long-duration transient flow regime. Nevertheless, these new DCA methods are still based only on production rate data, relying on the assumption of stable flowing pressure. Since this stabilized state is not reached rapidly in most cases, the 
applicability of these methods and the reliability of their solutions may be compromised. Examples of such situations are unconventional wells with short or discontinuous production histories, variable operating conditions and high pressure rate controlled wells. In these particular cases, important reservoir productivity signals are expressed through both pressure and rate decline trends. Therefore, ignoring flowing pressures and using only conventional ratetime techniques to interpret these cases may cause misinterpretations and erroneous reserves estimations (Anderson, D.M. et al., 2012; Mattar and Anderson, 2003).

Several flow regimes may be developed by multi-fractured horizontal wells in unconventional plays as a product of the multiple parameters in these complex reservoir/fractures systems (Clarkson, 2013; Clarkson and Pedersen, 2010; Liang et al., 2012; Luo et al., 2010; Mattar and Anderson, 2003). Because the dominant early flow regime is linear flow-followed by boundary-dominated flow-new DCA models specially designed for transient (linear) flow have been advocated. Furthermore, modifications have been introduced in those models to improve their ability to switch from linear to boundary-dominated flow models at an appropriate time (Joshi and Lee, 2013). However, in practice, decline curve analysis is often carried out without a proper identification of flow regimes. The arbitrary application of DCA models regardless of existing flow regimes may produce unrealistic production forecasts.

\subsection{Status of the Question}

An advanced generation of more complex techniques-known as rate transient analysis-is available to overcome with the restrictions in DCA mentioned previously. The main difference is that these techniques use analytical and numerical models to analyze production rates and flowing pressures. Although those methods are more rigorous and provide some advantages over DCA techniques, a deep understanding of reservoir characteristics and properties is required (some of which are known only approximately and others completely unknown). Considering that there is usually high uncertainty associated with most of the reservoir properties needed to use these complex methods, high uncertainty should 
correspondingly be expected in forecasts using this approach. Moreover, as the advanced analytical and numerical models depend on copious inputs, there is a high probability that different combinations of those parameters could generate equivalent and acceptable history matches, but different production forecasts and EUR estimations. Therefore, non-unique solutions may be expected (Anderson, D.M. et al., 2012).

One important feature of rate transient analysis is the application of the pressurenormalized-rates concept, which incorporates the effects of back pressure changes into the reservoir analysis (Mattar and Anderson, 2003). In spite of its advantages, the application of the pressure-normalized-rate concept has been mainly limited to rate transient analysis and has not been extended to decline curve analysis. Duong (2010) proposed a new decline analysis model suited for transient flow regimes. As part of his workflow, he included pressure corrected rates in diagnostic plots to compensate for the early choke-back effect and to improve identification of the linear flow regime, but not to improve the forecasting capacity of the model. So, Duong's model relies only on production rates.

Anderson, S. et al. (2012) proposed a new decline curve analysis method based on pressure-normalized rates to estimate ultimate recoveries and future production of high pressure, rate restricted wells. This method is grounded in the empirical observation that some wells exhibit a linear relationship between pressure-normalized rates and cumulative production when plotted on a semi-log scale; i.e., harmonic decline. Therefore, production is forecasted by extrapolating the harmonic decline of the pressure-normalized-rates. Although this is the first published DCA method to include normalize rates in the analysis, its application may be restricted to some specific cases, like high pressure, rate restricted wells exhibiting harmonic decline. Additionally, this method assumes that harmonic decline will continue during the entire well life. In fact, there is no physical concept supporting this assumption of harmonic decline. In conclusion, there is currently no clear methodology or technique to overcome the main restrictions in conventional decline curve analysis. Recent efforts have been focused on optimizing the switch of DCA models from linear to BDF, to obtain better representations of the most common flow regime sequence in unconventional reservoirs. However, two other 
important assumptions/restrictions continue without being properly addressed: lack of pressure data and stable operating conditions.

\subsection{Research Objectives}

- Evaluate the applicability of pressure-normalized decline curve analysis (PN-DCA) as a production forecasting technique suited for the interpretation of unconventional wells flowing under unstable operating conditions.

- Determine whether flow regime misinterpretations occur when diagnostic plots are not applied correctly.

- Compare the accuracy of the modern decline curve analysis models with varying amounts of historical production data.

- Assess the forecasting capacity of the pressure-normalized harmonic decline method.

\subsection{Expected Benefits and Application}

The results of this work should provide a better insight of the possible benefits provided by including flowing pressures in production decline analysis. As the pressurenormalized decline curve analysis (PN-DCA) relies on rates and pressure data, an improved forecasting ability is expected, especially in specific situation such as unstable operating conditions, limited availability of production data (short production history) and high-pressure, rate-restricted wells. Improved forecasting ability implies greater accuracy in the production performance forecasts and more reliable reserve estimations. The petroleum Industry may become more confident in reserves estimates, which are the basis for the optimization of development plans, future investments and estimates of companies' values. 


\section{DECLINE CURVE ANALYSIS}

Decline curve analysis is defined as "a graphical procedure used for analyzing declining production rates and forecasting future performance of oil and gas wells" (Aderemi and Akpara, 2008). Basically, a history match is developed by fitting a decline curve model onto past production data. Later, based on the new adjusted model, the existing production profile is extrapolated until it reaches a minimum economic rate or time limit, in order to forecast possible production performance and the expected ultimate recovery (EUR). Decline curve analysis (DCA) is commonly used and is considered a very powerful and efficient tool because high-quality results can quickly be obtained with a simple procedure, since it doesn't require sophisticated interpretation software or knowledge of the reservoir and well parameters. The concept of decline curve analysis was publicized by Arps (1945) and its application as a predicting method quickly spread as a regular practice in the oil and gas industry. Even today, when other modern methods (e.g., analytical and numerical models) have been created, it continues being a preferred forecasting technique.

Because DCA is an empirical technique based only on historical production rate trends to predict future production performance, it is fundamentally considered to lack a basis in physical principles governing the fluid flow phenomena in the reservoir. Fetkovich (1980) demonstrated that in some cases decline, curve analysis has solid fundamental bases and can be expressed in terms of reservoir variables and engineering concepts. He proved that under specific conditions (constant pressure and slightly compressible single phase radial flow systems), the empirical exponential decline model is equal to the analytical solution. Although Fetkovich's work clarified the relation of the DCA concept with the fluid flow physics, it is strictly restricted to very specific cases. Therefore, DCA is still considered as an empirical approach based on production observations. By virtue of its empirical nature, decline curve analysis can be applied to production data from any type of reservoir, independently of fluid and rock properties, drive mechanism and operating conditions. Additionally, the analysis could 
also be performed on any fluid stream, including a single fluid (gas, oil or water), multiple fluids, or production ratios (WOR, WGR, GOR). The practical procedure is to select a production variable showing a recognizable trend that can be used to develop an optimum and reliable forecast. Usually, production decline analyses are conducted on a plot of rate-versus-time or rate-versus-cumulative production, and as a result, it can predict remaining recoverable reserves, but not fluids-in-place.

Arps' decline model, also known as basic or traditional DCA, are grounded in the concept that boundary-dominated flow is the dominant flow regime and that operating conditions and reservoir properties remain constant. Therefore, decline curve analysis assumes that reservoir boundaries have been reached and that the main factors involved in the production decline process will remain constant during the future well life. If production conditions are changed, well performance is altered and the forecast analysis is no longer valid. Some of the factors affecting production performance are pressure depletion, number of producing wells, drainage area, drive mechanism, reservoir characteristics, skin, saturation changes, and relative permeability, as well as operating conditions such as separator pressure, tubing size, choke setting, workovers, compression, operating hours, and artificial lift (Fekete, 2013b). It is important to keep in mind that special operations, like infill drilling, formation stimulation or wellbore reconfiguration, can change the reservoir performance by reducing reservoir drainage area, altering productivity properties and effective fluid flow areas.

In general, DCA techniques are extremely valuable tools for production analysis because of their simplicity. However, some of those features that make them simple also represent a disadvantage in other aspects:

- Lack of pressure data. According to Mattar and Anderson (2003), traditional decline curve analysis provides reasonable answers in many situations, but has its limitations, because it completely ignores the flowing pressure data.

- Boundary-dominated flow assumption. This condition is not readily reached in unconventional reservoirs (e.g., tight sands and shales) where the dominant linear flow regime can last several years before reaching stabilization. 
- Consistent production history is needed. Decline curve analysis may be applied only when sufficient and coherent historical production data is available to reliably identify a convincing decline trend.

- Invariable operating conditions. As previously described, one of the main restrictions of the decline curve analysis is its inability to disassociate the estimation of future production performance from the existing operation constraints under which production history was developed (Mattar and Anderson, 2003). This means that forecasts provided by DCA models may be considered reliable representative only if operating conditions remain unchanged.

- Non-uniqueness: Decline curve analysis does not provide unique solutions, especially when the curve fitting is performed over a short production history or when data scattering is significant. A poor quality history match allows the possibility of obtaining multiple combinations of potential decline parameters, which in turn will generate a wide range of EUR estimations.

In recent years, as a result of the increasing exploration and production activity in unconventional plays, new decline curve models-also known as advanced decline curve models-have been developed. The main purpose of these new models (e.g., e.g., SEPD, Duong, Logistic Growth, Power Law, and Dual Models) is to overcome the boundary-dominated flow assumption of the basic Arps' models, which restricts its application in ultra-low permeability reservoirs, exhibiting long-duration transient flow regime.

\subsection{Basic Decline Curve Analysis}

\subsubsection{Arps Decline Equations}

Arps (1945) presented a set of empirical equations to forecast production performance. Those equations are based on the concept of loss-ratio (Eq. 1) and its derivative (Eq. 2). The inverse of the loss-ratio is known as the decline rate (D) and represents the fractional change of rate per time-usually expressed in \%/year. 


$$
\begin{aligned}
& \frac{1}{D}=\frac{-q}{d q / d t} \ldots \ldots . . . \\
& b=\frac{d}{d t}\left(\frac{-q}{d q / d t}\right) .
\end{aligned}
$$

The general form of the Arps models is represented by Eq. 3:

$$
q=q_{o}\left(1+b D_{i} t\right)^{-1 / b}
$$

However three different decline models can be subsequently derived from this general equation depending on the different combinations of the production decline parameters $(b$ and D), as presented below.

\subsubsection{Exponential}

Exponential decline occurs when the decline rate, $D$, is constant and the $b$ exponent is 0 . This model provides the minimum EUR, so it is the most conservative, as well as the simplest basic decline curve. Eqs. 4 and 5 presents the exponential expression for production rates and cumulative production, respectively.

$$
\begin{aligned}
& q=q_{o} * e^{(-D t)} \cdots \\
& Q=\frac{q_{o}}{D}\left[1-e^{D t}\right]
\end{aligned}
$$

\subsubsection{Hyperbolic}

The hyperbolic model is equivalent to the general form of the Arps equation. If $D$ is changing on time, the decline is considered to be either hyperbolic or harmonic. The hyperbolic exponent $b$ represents the varying decline rate. Eqs. 6 and 7 presents the hyperbolic expression for production rates and cumulative production, respectively. 


$$
\begin{aligned}
& q=q_{o}\left(1+b D_{i} t\right)^{-1 / b} \\
& Q=\frac{q_{o}}{(1-b) D_{i}}\left[1-\left(1+b D_{i} t\right)^{1-(1 / b)}\right]
\end{aligned}
$$

In this case, the decline rate at any time, D, is expressed as presented in Eq. 8.

$$
D=\frac{D_{i}}{1+b D_{i} t}
$$

\subsubsection{Harmonic}

The harmonic model is a special case of hyperbolic declination when $b$ is equal to 1 . When a harmonic decline is performed, the lower part of the curve tends to become very flat with time, so that it appears no decline is happening and the production rate will never be zero. Therefore, it is not possible to estimate the maximum EUR (when production rate is zero), but a EUR at a specific abandonment rate can be predicted. This type of performance is expected when very effective recovery mechanisms, such as gravity drainage, are active. Another example of harmonic decline is the production of high viscosity oil driven by encroaching edgewater (Fekete, 2013b). Eqs. 9 and 10 presents the hyperbolic expression for production rates and cumulative production, respectively.

$$
\begin{aligned}
& q=\frac{q_{o}}{\left(1+D_{i} t\right)} \\
& Q=\frac{q_{o}}{D_{i}} \ln \left(1+D_{i} t\right)
\end{aligned}
$$

In the case of harmonic decline, the production profile is a straight line when plotting log flow rate vs. cumulative production (See Eq. 11):

$$
\log q=-\frac{D_{i} Q}{2.303 q_{i}}+\log q_{i}
$$

Fig. 1 illustrates the behavior of the three rate-time relationships proposed by Arps. 


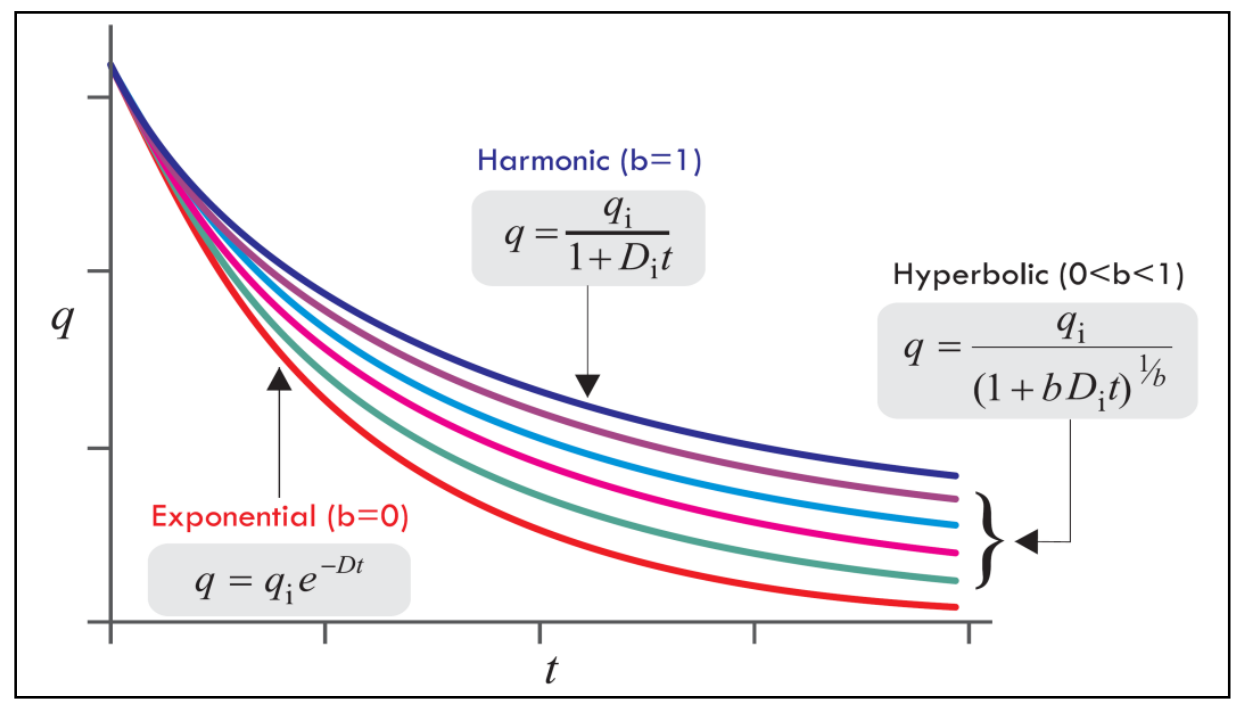

Fig. 1-Traditional Arps Decline Curves (Fekete, 2013a).

\subsubsection{Hyperbolic Exponent " $b$ "}

The hyperbolic exponent " $b$ ", also known as the loss ratio (inverse of decline rate) derivative, depicts the change of the production decline rate over time (Eq. 12):

$$
b=\frac{d}{d t}\left(\frac{1}{D}\right)=\frac{d}{d t}\left(\frac{-q}{d q / d t}\right)=\frac{n d_{i}(1-n) t^{(n-2)}}{d^{2}}
$$

Arps analyzed production data from 149 fields and, as a result of his observations, he found that the range of exponent " $b$ " was between 0 and 0.7 with $90 \%$ having $b$ less than 0.5 . No harmonic declines were found and less than $15 \%$ had a b value less than 0.1 (Fetkovich et al., 1996). Several expected values of $b$ were derived for different reservoir drive or recovery mechanisms. Table 1 shows the " $b$ "values that should be expected for homogeneous singlelayer or layered crossflow systems, while Fig. 2 illustrates those observations. In general, the higher the $b$ value, the stronger the secondary energy source. 
Table 1-Decline exponents " $b$ " for various reservoir mechanisms (Fekete, 2013b)

\begin{tabular}{|c|l|}
\hline b value & \multicolumn{1}{|c|}{ Reservoir Drive Mechanism } \\
\hline 0 & Single phase liquid expansion (oil above bubble point) \\
& Single phase gas expansion at high pressure \\
& Poor waterflooding performance \\
& Tubing restricted gas production \\
\hline $\mathbf{0 . 1}-\mathbf{0 . 4}$ & Solution gas drive \\
\hline $\mathbf{0 . 4 - 0 . 5}$ & Single phase gas expansion (Typical gas well) \\
\hline $\mathbf{0 . 5}$ & Effective edge water drive \\
\hline $\mathbf{0 . 5 - 0 . 9}$ & Commingled layered reservoirs \\
& Gas or water injection \\
\hline $\mathbf{1 . 0}$ & Very active water drive or gas cap drive \\
\hline & Gravity drainage \\
\hline
\end{tabular}

In some situations, such as in the case of ultra low permeability shales or tight sands, decline curve analysis shows " $b$ " values higher than 1. As pointed out by Fetkovich et al. (1996) "attempting to fit all or some of the transient production rate data of a well with the Arps pseudosteady-state equation will result in an apparent b value higher than it really is. In some cases, it will even be greater than 1". Production performance in wells producing from unconventional reservoirs is dominated by long linear flow periods, where " $b$ " is not constant. This topic will be discussed in detail in the next section.

According to Kanfar and Wattenbarger (2012), Arps equations with " $b$ " values of 2 and 4 represent either bilinear or linear flow respectively. Nevertheless, basic Arps equations are not suitable to model transient flow or multiple flow regimes. So, EUR calculations should be addressed with that basic method only when boundary-dominated flow regime is observed. 


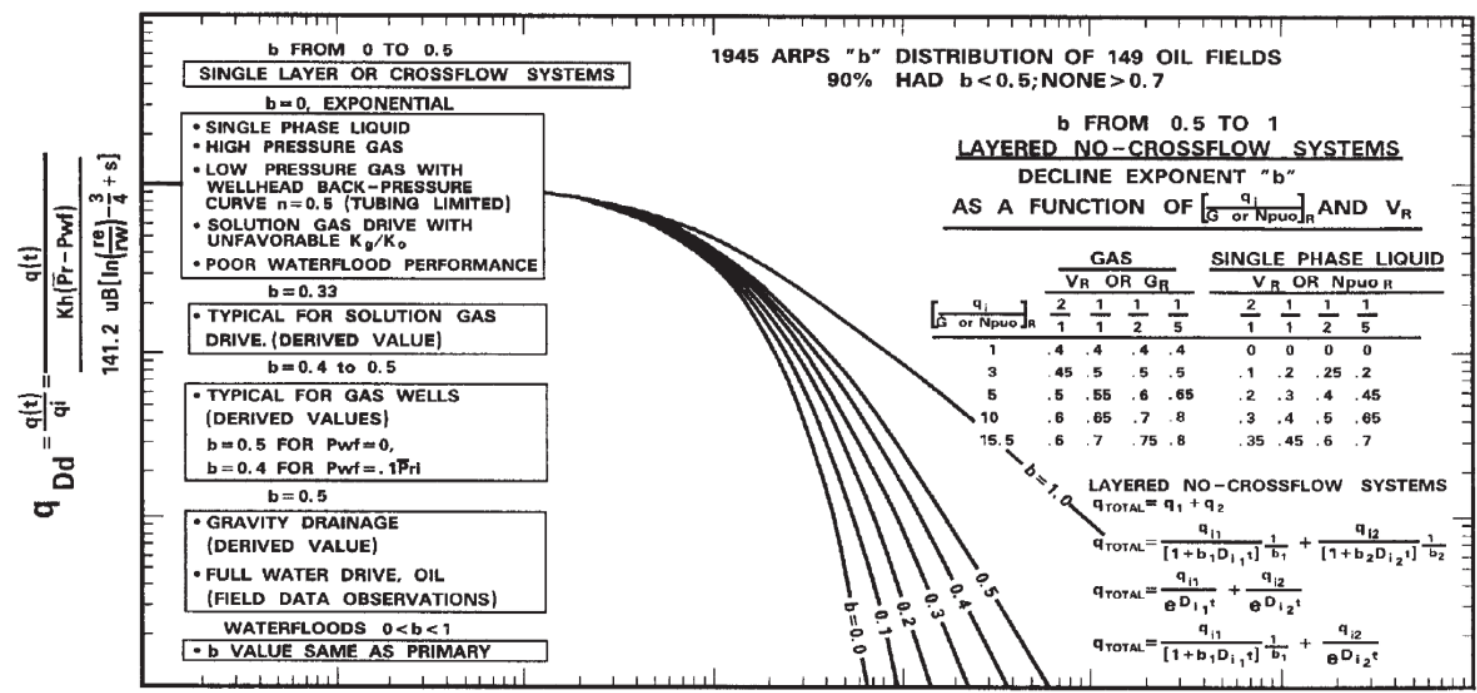

$$
t_{D d}=D_{i} t=\left\{\begin{array}{l}
\text { GAS }=\frac{1}{(1-b)} \frac{q i}{G} t=2 n\left(\frac{q i}{G}\right) t \\
\text { OIL }=\frac{1}{(1-b)} \frac{q i}{N p o_{0}} t=\frac{2 n+1}{2}\left(\frac{q i}{N p u o}\right) t
\end{array}\right.
$$

Fig. 2-Decline exponents "b" for various reservoir drive mechanisms (Fetkovich, 1996).

Thompson et al. (2011) found that in those shale wells where desorption plays a significant role in the fluids storage and production performance, the late time " $b$ " parameter may be affected and its value may increase due to the additional energy provided by the desorption capacity of the rock. As consequence, EUR estimations increase when desorption effect is included in the analysis.

\subsection{Advanced Decline Curve Analysis}

\subsubsection{Application Of Decline Curve Analysis To Unconventional Reservoir}

As previously mentioned, the ultimate recovery of conventional reservoirs can be reliably estimated using traditional Arps' decline analysis equations, which assume boundarydominated flow (BDF) as dominant flow regime. But this is not the case of unconventional reservoirs, which frequently exhibit long transient flow regimes and don't reach BDF for most 
of their production life due to the very low matrix permeability-usually in the range of nanoDarcies-(Kanfar and Wattenbarger, 2012). Long transient flow periods mean changing drainage area, while Arps DCA models assume constant drainage area and flowing pressure. When Arps curves are used to fit shale wells production data, " $b$ " values higher than 1 are obtained, and extrapolation of those models often forecast infinite unrealistic reserves (Anderson, D.M. et al., 2012).

Initially, during transient flow, the decline rate is high and the production rate steeply decreases, but it stabilizes when boundary-dominated flow is reached. For most wells, especially in conventional reservoirs, this happens within a few months of production. However, transient flow conditions can last several years in very low permeability wells (Fekete, 2013b). Actually, in some cases, wells are abandoned before reaching any drainage boundary.

Those problems led to the development of new empirical equations more suitable for production analysis in unconventional reservoirs. The main strength of this new generation of decline curve models is its great ability to generate efficient solutions to complex unconventional reservoir systems by just applying simple time-rate relations (Mangha et al., 2012). In practice, they share most of the features of basic decline analysis techniques, with the exception of the boundary-dominated flow restriction. This new set of advanced decline curve methods overcome this problem by introducing a new way of adjusting the changing decline parameter. They better model the transition from transient into boundary-dominated flow regime after an estimated time or upon achieving a certain region of investigation. In general, the fundamental concept of this modification is grounded in the assumption that depletion will dominate the production performance as soon as transient flow ends (Anderson et al., 2010). As well as in the case of the traditional Arps equations, there may be an analytical theory to explain the basis of these new models, but at present it has not be demonstrated. Thus, they are considered completely empirical (Mangha et al., 2012).

Some of the most popular advanced decline curve models are:

- Power-Law Exponential Model (Ilk et al., 2008) 
- Stretched Exponential Model (Valko and Lee, 2010)

- Logistic Growth Model (Clark et al., 2011)

- Duong Model (Duong, 2010)

It is important to mention that decline curve methods, as well as other forecasting techniques, do not provide an unique solution because of the difficulty of predicting the influence of the multiple reservoir variables affecting production performance. Additionally, according to (Mangha et al., 2012), it is difficult to have one single rate-time model that accurately works for all the different unconventional plays-one model could be adequate for one play, but possibly not for another one. When analyzing production data, all the available models should be considered, so it is critical to have a good understanding of each of those methods.

Additionally, Mangha et al. (2012) presented a workflow to analyze and forecast ratetime data of wells in low/ultra-low permeability reservoirs. The key component of the proposed workflow is the application of the "Db," " $\beta$-derivative," and "q/Gp" diagnostic plots as an analysis guide to obtain model parameters for the time-rate models. After obtaining the model parameters, the production profile can be extrapolated to generate the "estimated ultimate recovery" (EUR) at a specified time limit or abandonment rate. This methodology is based on the concept that diagnostic plots provide direct insight into our understanding of decline behavior, ensuring a more comprehensive and representative analysis. Competent results are obtained when sufficient production history is available.

The next sections provide a practical insight of the most important models proposed in the literature and applied by the industry:

\subsubsection{Stretched Exponential Production Decline Model (SEPD)}

This method, proposed by Valko and Lee (2010), is a new form of the hyperbolic model with a varying " $\mathrm{b}$ " exponent over the production time, deducted from the observation of a large group of wells with production profiles exhibiting a stretched exponential decline trend. 
According to Valko and Lee (2010), SEPD model offers two important advantages. The first one is its ability of forecasting bounded ultimate recoveries (EUR) without requiring the definition of any rate and/or time limits, producing more realistic reserve estimates for unconventional reservoirs. Second, the recovery potential relation (RP) as function of cumulative production can be obtained.

The principles behind the stretched exponential model assumes that "the actual production decline is determined by a great number of contributing volumes individually in exponential decay (i.e., in some kind of pseudo-steady state), but with a specific distribution of characteristic time constants", mentioned by Valko and Lee (2010).

The SEPD model includes two new parameters $(n, \tau)$. In general, the " $\tau$ " parameter is the median of the characteristic time constants and " $n$ " is the exponent parameter. The closer $\mathrm{n}$ is to zero, the larger the distribution tail. (Valko and Lee, 2010).

The following equation corresponds to the SEPD relations to calculate production rate (Eq. 13), decline rate (Eq. 14), cumulative production (Eq. 15), EUR (Eq. 16) and recovery potential (Eq. 17).

$$
\begin{aligned}
& q=q_{o} \exp \left[-\left(\frac{t}{\tau}\right)^{n}\right] \\
& D=\frac{n t^{(n-1)}}{\tau^{n}} \\
& Q=\frac{q_{o} \tau}{n}\left\{\Gamma\left[\frac{1}{n}\right]-\Gamma\left[\frac{1}{n},\left(\frac{t}{\tau}\right)^{n}\right]\right\} \\
& E U R=\frac{q_{o} \tau}{n} \Gamma\left[\frac{1}{n}\right] \\
& R P=1-\frac{Q}{E U R}
\end{aligned}
$$


In the SEPD cumulative equation, the first term inside the brackets is the complete gamma function, while the second one is the incomplete gamma function, as defined by Abramowitz and Stegun (1964). Gamma function is shown in the Eq. 18:

$$
\Gamma[a, x]=\int_{x}^{\infty} t^{a-1} e^{-t} d t
$$

Once positive values of $n, \tau$ and $q_{0}$ are given, finite values of EUR are obtained, even if rate and/or time limits are not specified. As pointed out by Kanfar and Wattenbarger (2012), unlike the PLE model, SEPD does not consider a late time term $-D_{\infty}$ is always considered to be zero. Additionally, the SEPD model provides a cumulative production function, what is considered an important advantage. Because of the smoothness of cumulative production data in comparison with noisy rate data, the SEPD model may be easier fitted when its cumulative production function is used instead of its rate function. As well as in the PLE method, a representative EUR estimation can be obtained if a meaningful portion of BDF is present (Freeborn and Russell, 2012).

This model is complex and difficult to solve, so it tends to be underutilized in spite of its great advantages. Freeborn and Russell (2012) presented a practical guideline to better understand and easily apply this model, as well as an error minimizing algorithm to determine the value for the " $n$ " exponent. He also suggests that the Power Equation and the Stretched Equation provide similar solutions and that the most practical approach is to apply both models in concert and to select the most conservative forecast.

\subsubsection{Duong Model}

Duong's method is based on the consideration that most of the production from ultralow permeability unconventional reservoirs (super-tight sands or shales) is mainly carried out by the fracture network, in such a way that linear flow is the dominant regime for several years. In fact, some of these wells are abandoned even before reaching a pseudo-steady state (BDF), so neither permeability nor drainage area can be estimated. This long transient flow performance is additionally supported by the theory that the connected fracture network in the 
stimulated region (SRV) increases over time due to local stress variations caused by depletion of the fractures-i.e., reactivation of existing fractures/faults because of pressure depletion, matrix shrinkage and effective stress changes. Since matrix contribution is insignificant in comparison with fracture network contribution, EUR cannot be established by traditional methods based on the drainage area concept (Duong, 2010). These observations led Duong to develop an alternative approach to forecast production performance and estimate EUR for fracture dominated wells.

In general, Duong's model is grounded on the concept that the rate-time function, material balance time $(Q / q)$ vs. time, exhibits a power law relation (as presented in the Eq. 19) forming a straight line when plotted on a log-log scale (See Fig. 3). However, note that when BDF is reached, the production trend may deviates from the log-log straight line (Kanfar and Wattenbarger, 2012). In Eq. 19, " $m$ " represents the slope and "a" is the intercept. Although slope is always negative, " $\mathrm{m}$ " value is always positive and greater than unity for unconventional reservoirs. If " $\mathrm{m}$ " is less than unity, it might indicate a conventional low permeability reservoir (Duong, 2010).

$$
\frac{q}{Q}=a t^{-m}
$$

Even though two different flow regimes usually are developed in fracture dominated production systems (bilinear flow in finite conductivity fractures, exhibiting a quarter-slope line on a log-log plot of production rate vs. time, and linear flow in infinite conductivity fractures, indicated by half slope line on the same plot), Duong showed that both cases exhibit a slope close to -1 in a log-log plot of $q / G p$ versus time (Mishra, 2012). Fig. 3 shows log-log plots of $q / G_{p} v s$. $t$ for various shale gas wells with more than 5 years of production. 


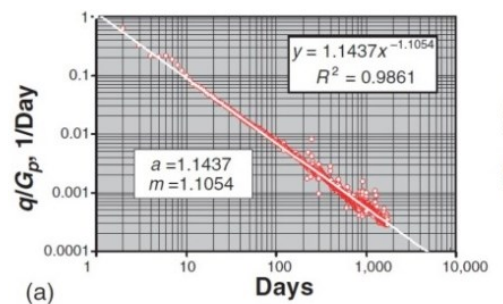

(a)

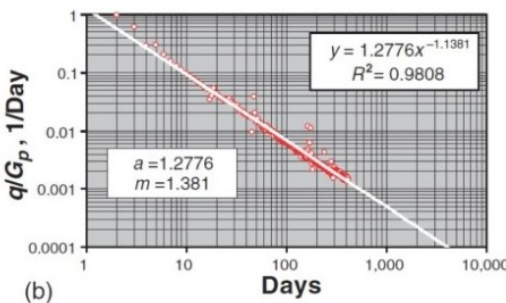

(b)

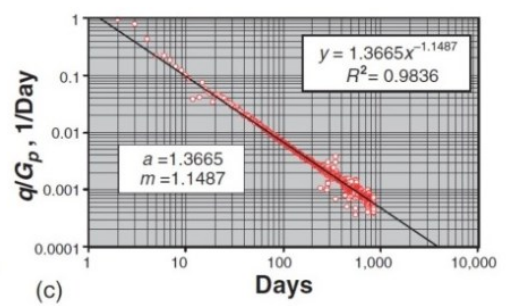

(c)

Fig. 3-q/Gp vs t plot for varios shale gas wells (Duong, 2010).

The Duong's equations for rate (Eq. 20), cumulative production (Eq. 21), EUR (Eq. 22) and time function (Eq. 23) are presented below:

$$
\begin{aligned}
& q=q_{1} t(a, m)+q_{\infty} \\
& Q=\frac{q_{1} t(a, m)}{a t^{-m}} \\
& E U R=\frac{q_{e c o}}{a} t_{e c o}^{m} \\
& t(a, m)=t^{-m} \exp \left[\frac{a}{1-m}\left(t^{1-m}-1\right)\right]
\end{aligned}
$$

The second term in the rate equation $\left(q_{\infty}\right)$ was added to get a better fit of the model in some field cases where a straight line doesn't intercept the origin in the q vs. $t(a, m)$ plot. This term can be negative or positive. Since the cumulative production equation was not deducted considering this term, this should not be used if $q_{\infty} \neq 0$. Otherwise, an inaccurate EUR estimation might be produced (Kanfar and Wattenbarger, 2012).

According to Freeborn and Russell (2012), it is a very attractive method given its simplicity (i.e., easy to understand and apply). Nevertheless, since this method is founded on the production extrapolation for one single flow regime, it should be considered a poor forecasting tool when multiple flow regimes are shown. So, this model should be suitably applied only while linear flow lasts (Freeborn and Russell, 2012). 
Similar to other DCA methods, if a well is shut in for a significant time period, a rate initialization may be required. Additionally, some factors (water production, liquid holdup in wellbore and/or fracture network) may cause the deviation of flow behavior from linear flow, accelerating the rate decline (Duong, 2010).

As pointed out by Mangha et al. (2012), unlike of those models that don't exhibit linear behavior-such as power-law exponential, stretched exponential, and logistic growth-Duong's model is a linear based model (q/Gp-t data trend on a log-log scale) and its EUR estimates may be higher or optimistic in comparison with the results from those other models. Some models, such as modified-hyperbolic and power-law exponential, include a late time term which diverges its behavior from the linear trend and constrains EUR over-estimations, while Duong's model not.

\subsubsection{Power Law Exponential Method}

This technique, proposed by Ilk et al. (2008), was derived from their observations on the behavior of the loss ratio function and its derivative, and is based mainly on modeling the power law trend of the D-parameter with time (i.e., straight line on log-log scale). The fundamentals of this model suggest that the loss ratio can be represented by a decaying power law function with a constant behavior at large times and that the loss ratio derivative (bparameter) does not exhibit a constant trend as assumed in the traditional hyperbolic model. As stated by Ilk et al. (2011), this power-law behavior is a common feature of unconventional reservoirs.

As presented in Section 2.1.1, the loss ratio (1/D) and its derivative (b) are defined as presented in Eqs. 24 and 25:

$$
\begin{aligned}
& \frac{1}{D}=\frac{-q}{d q / d t} \\
& b=\frac{d}{d t}\left(\frac{-q}{d q / d t}\right)=\frac{n d_{i}(1-n) t^{(n-2)}}{d^{2}}
\end{aligned}
$$


An alternate calculation of these two parameters using rate-cumulative data instead of rate-time data was provided by Ilk et al. (2008), as presented in Eqs. 26 and 27:

$$
\begin{aligned}
& D=-\frac{d q}{d Q} \ldots \\
& b=q \frac{d}{d Q}\left(\frac{1}{D}\right)
\end{aligned}
$$

The Power Law D-parameter model (loss ratio) is defined as presented in Eq. 28. The first term of this equation $\left(D_{\infty}\right)$ is a constant and corresponds to the loss-ratio at infinite time, while the second one is the time-dependent power law function (Kanfar and Wattenbarger, 2012).

$$
D=D_{\infty}+D_{1} t^{-(1-n)}
$$

According to Ilk et al. (2008), this equation is totally flexible and offers the advantage of modeling multiple flow regimes (transient, transition, and boundary-dominated flow) because the "b hyperbolic exponent varies with time. However, it has the special feature that at late times the relation behaves as the basic exponential decline model.

Fig. 4 shows a schematic plot for hyperbolic and power law exponential models for orientation purposes. It offers a clear comparison between the traditional hyperbolic and the power law models. It can be readily seen that the D-parameter of the traditional hyperbolic model is practically constant at early times and decays as a power law function at late times. Meanwhile, the power law model behaves in an opposite way, such that at early times it decays as a power law function and then turns gently towards a constant value (Ilk et al., 2008). It happens because at early times the offset term $\left(D_{\infty}\right)$ is negligible, so the model performs as a power law function from transient through transition flow, matching linear or bilinear flow (Kanfar and Wattenbarger, 2012). Finally, at late times the first term dominates and the loss ratio is constant, representing the boundary-dominated flow regime and behaving as an exponential rate decline model. 


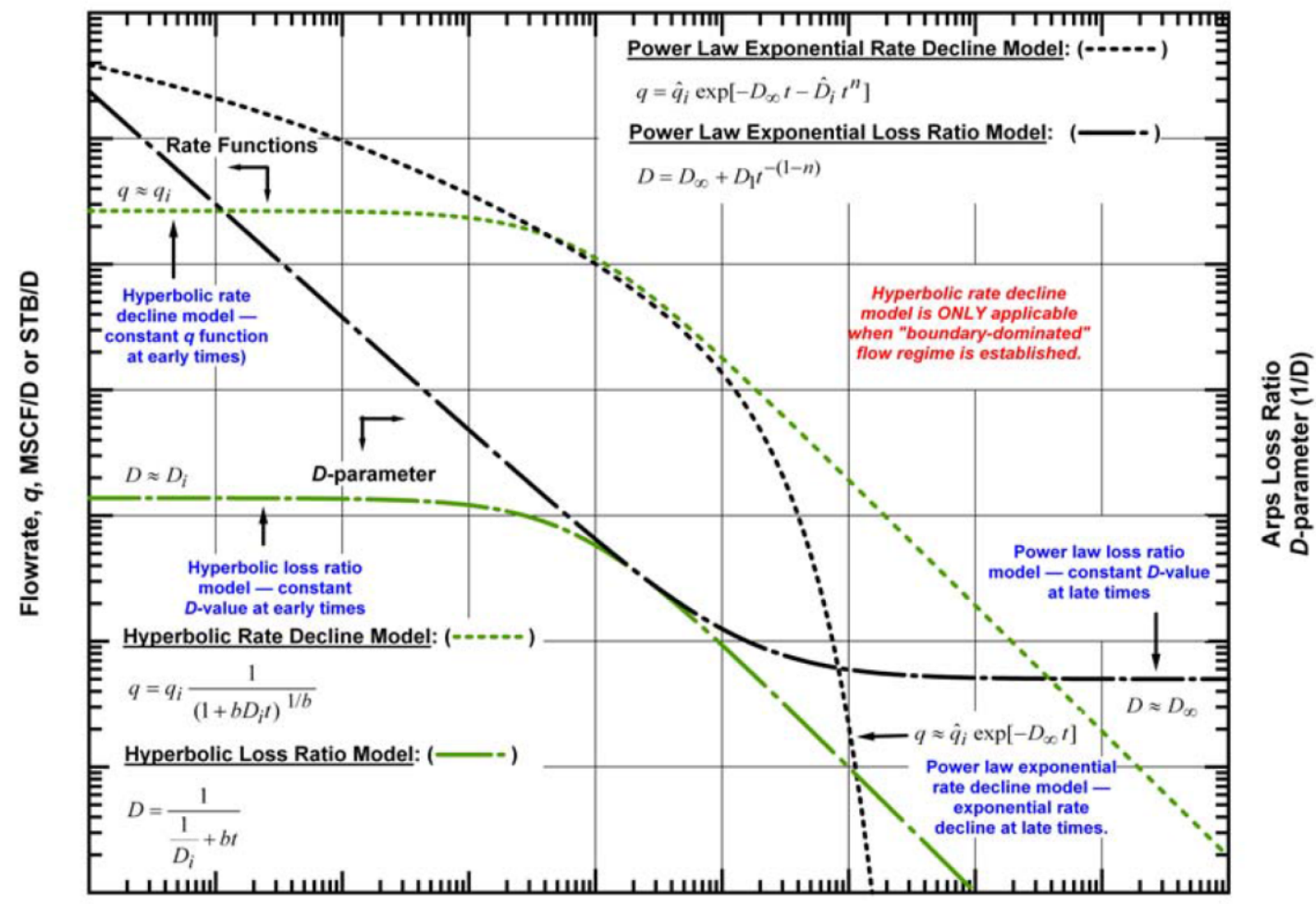

Production Time, $t$, days

Fig. 4-Schematic plot for hyperbolic and power law exponential models (Ilk et al., 2008)

The Power law rate-time relation is defined as presented in Eqs. 29 and 30:

$q=\hat{q}_{i} \exp \left[-D_{\infty}-\widehat{D}_{i} t^{n}\right]$

$\widehat{D}_{i}=D_{1} / n$

Since it is not possible to integrate this rate-time relationship, it is necessary to estimate cumulative production by numerical integration. The Power Equation is solved by multivariate linear regression when the " $n$ " time exponent value is known. Freeborn and Russell (2012) provides an error minimizing algorithm to determine the " $n$ " value and some practical indications about the PLE solution. As stated by Freeborn and Russell (2012), the PLE 
equation doesn't have the ability of neither identifying the occurring flow regimes nor predicting the BDF onset. Instead, the PLE D-parameter allows switching to a terminal decline rate when enough historical BDF production data is available.

\subsubsection{Logistic Growth Model}

Logistic Growth Models (LGM) is a set of mathematical models commonly used to predict growth in different disciplines. Their main feature is the carrying capacity term (K), which limits the population growth limit or define the maximum population size at which growth rate will finish (Clark et al., 2011). A particular logistic growth model specially adapted for oil and gas production forecasting purposes was developed by Clark et al. (2011). Eq. 31 corresponds to the rate-time expression, while Eq. 32 is the cumulative production function.

$$
\begin{aligned}
& q=\frac{K n a t^{n-1}}{\left(a+t^{n}\right)^{2}} \\
& Q=\frac{K t^{n}}{a+t^{n}} \ldots . .
\end{aligned}
$$

In this case, the carrying capacity term (K) represents the maximum recoverable volume, independent of time or economic restrictions, so it is close to the EUR without considering any economic analysis. As the carrying capacity term is the restriction parameter that controls the rate decline up to reaching the maximum cumulative production, it is recommended to try to determine EUR in a different way prior to performing a production forecast. When EUR is unknown, " $\mathrm{K}$ " and the other LGM parameters can be determined by matching the historic production data (Clark et al., 2011). Unfortunately, LGM is a very flexible model, so multiple solutions can be obtained with different combinations of parametersincluding the carrying capacity term $(\mathrm{K})$ that define the maximum cumulative production. The Fig. 5 illustrates a production data fit with the LGM technique without a previous knowledge of the carrying capacity. Multiple good fits were obtained, as well as multiple EUR estimations. Moreover, the logistic growth model, just like the power-law exponential and the stretched 
exponential relations, tends to generate conservative production forecasts (Mangha et al., 2012).

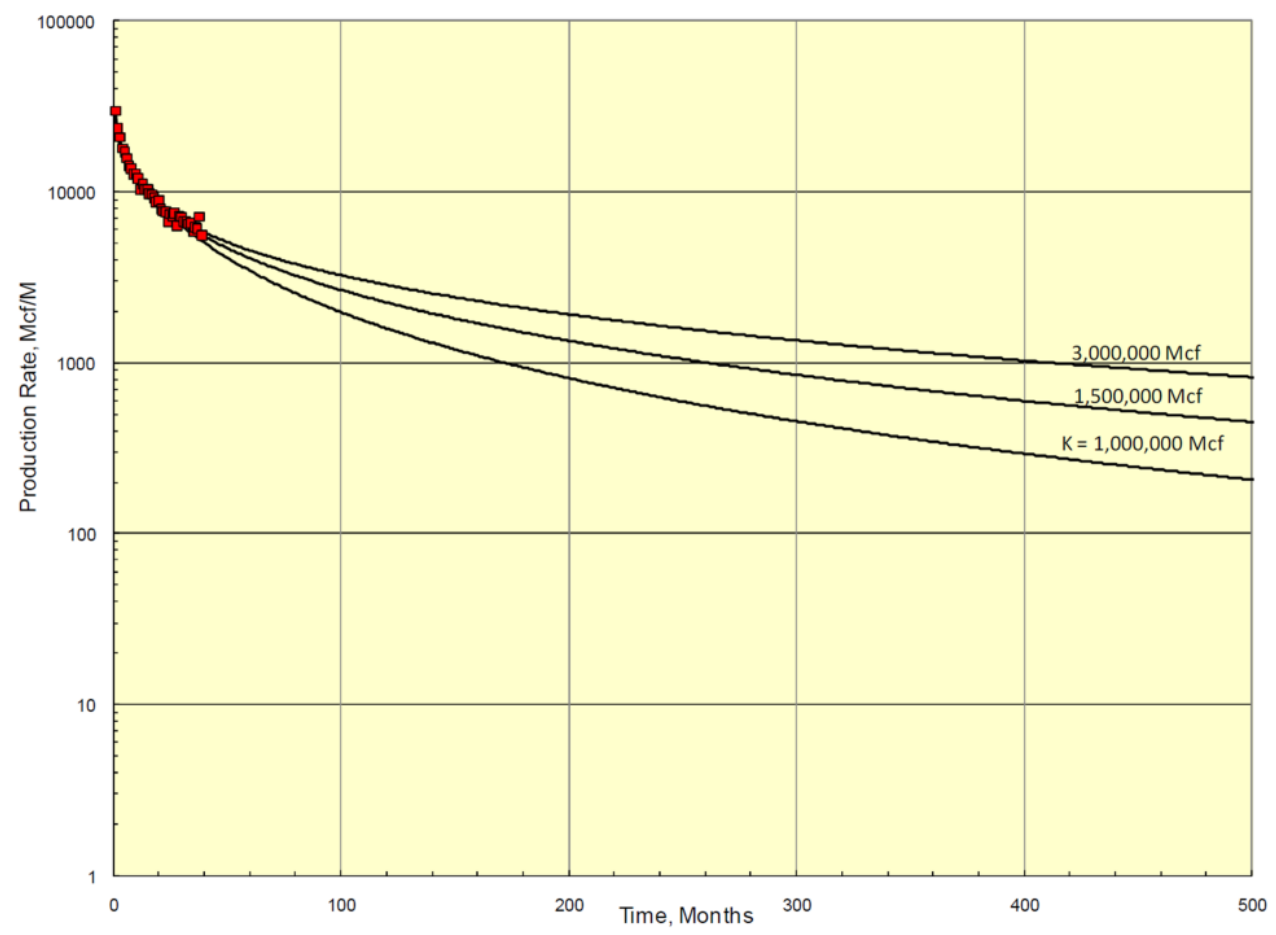

Fig. 5-Production data analysis by applying LGM (Clark et al., 2011)

\subsubsection{Dual Models}

Dual models are compound models resulting from the combination of two different single models-e.g., Duong, SEPD, Hyperbolic, Exponential-where each of these models has the advantage of better forecasting production performance during a specific flow regime. For unconventional reservoirs, the best dual-model configurations are those with the ability of modeling linear flow during the early well lifetime, and later switching into a BDF model when a limiting condition is reached-i.e., certain time or a minimum decline rate. As pointed out by 
Freeborn and Russell (2012), the switching point $\left(t_{s w}\right)$ represents the transitions from linear to boundary-dominated flow. Selecting this point is very important because it has a large effect on the production model behavior and on the subsequent EUR forecast. In most occasions, identifying the BDF onset is very difficult or just impossible. Therefore, one of the biggest uncertainties related to dual models is the precise selection of the switching point, especially when BDF is not evident. In these cases, it may be estimated from analog wells or could be an arbitrary number based on company's policies and/or the analyst's experience (Mangha et al., 2012). Because Arps models were designed to analyze production data in BDF conditions, these are considered the best terminal decline models. Two of the most commons Arps' BDF models are either a hyperbolic curve with a b exponent between 0.3-0.4 (0.4 for gas wells or 0.3 for oil wells) or an exponential curve. Three of the most common dual models are presented below.

\subsubsection{Modified Hyperbolic}

In this case, an initial Arp's hyperbolic decline curve-usually with a b exponent higher than 1-switches into a terminal Arp's BDF model either at a specific time $\left(t_{\text {sw }}\right)$ or when a predetermined nominal decline is reached $\left(D_{\text {lim }}\right)$. This is a very practical and widely used way to constrain the EUR overestimations caused by fitting early-time transient data with the traditional hyperbolic equations and the resulting " $b$ " parameter higher than 1 (Ilk et al., 2011).

The Fig. 6 shows an illustration of the modified hyperbolic model used to forecast production for a shale gas well. This forecast includes 4 production scenarios resulting from fitting the same historic production data using 4 different models. The blue line corresponds to the extrapolation of the basic hyperbolic model $(b>1)$, while the other 3 scenarios correspond to the modified hyperbolic model at three different terminal decline rates $(12.5 \%, 19.0 \%$ and $30 \%)$. It can be observed that the basic DCA model brings about a production performance overestimation, while the modified models produce more conservative results. The transition point depends on the specified terminal decline and has a direct impact on the EUR forecast. 


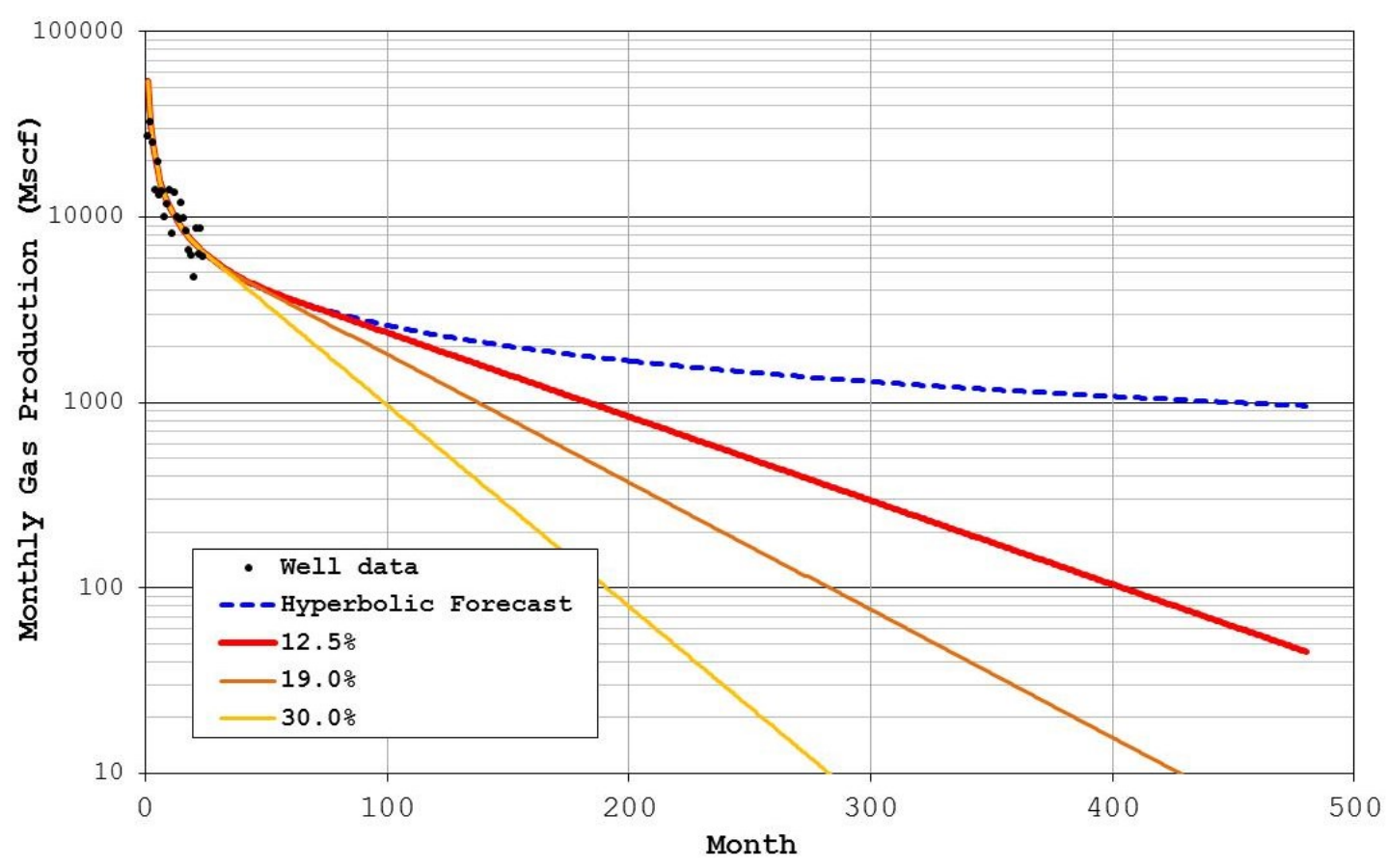

Fig. 6-Example of the Modified Hyperbolic Technique.

\subsubsection{Modified Duong}

As previously mentioned, the Duong method is a single flow regime model focused only on the transient flow regime, so it is not flexible enough to provide a solution for later BDF. Considering this constraint, some modifications to Duong's model have been proposed by Joshi and Lee (2013) in order to account for the BDF effects in long production wells or new wells with high fracture density and improved permeability in the stimulated reservoir volume (SRV). Additionally, they made important observations on the late time Duong's model term $\left(q_{\infty}\right)$ :

- Using a non-zero $q_{\infty}$ can produce unrealistic forecasts, especially when production history is short.

- In the case of short production history, the error can be minimized if the fitted line is forced to intercept the origin $\left(q_{\infty}=0\right)$. 


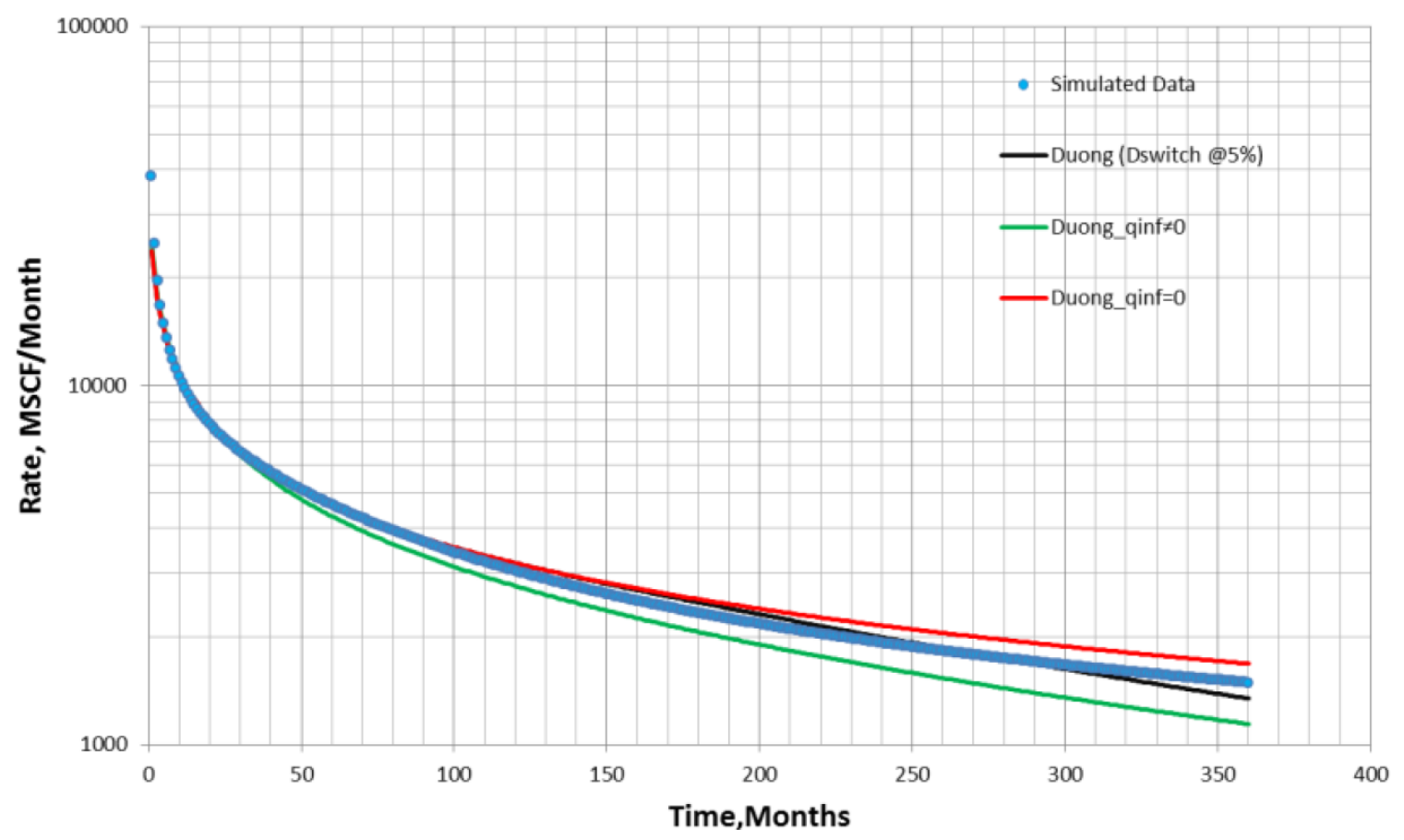

Fig. 7-Comparison of original Duong and modified Duong models (Joshi and Lee, 2013).

Considering these facts, two important modifications have been proposed by Joshi and Lee (2013):

- When applying the straight line method to establish " $q_{1}$ ", it should be forced to intercept the origin and avoid $q_{\infty} \neq 0$, especially when short historic production data is available (Less than 24 months approximately).

- As the Duong model assumes transient flow and Arps models assume BDF, the best solution is to use a dual model that switch from Duong to Arps after certain time or when a defined rate is reached-ideally at the BDF onset. In their study a switch point equal to $5 \%$ of declination rate $\left(D_{\text {switch }} @ 5 \%\right)$ is proposed, but the optimal $D_{\text {switch }}$ and "b" values for the BDF Arps model should be selected through a rigorous study of each case. 
Fig. 7 illustrates the advantage of the modified Duong model over the original one. Here a comparison of the original Duong, the modified Duong and the Modified Duong $\left(D_{\text {switch }}\right.$ @ 5\%) is developed for a Barnett simulation case (48 months of history match).

\subsubsection{Modified Stretched Exponential}

Also known as MSE, this model switches from SEPD into a terminal Arp's BDF model when a limiting condition is reached, just like the modified Duong and the modified hyperbolic models do. 


\section{RATE TRANSIENT ANALYSIS APPLIED TO MULTI-FRACTURED HORIZONTAL WELLS IN UNCONVENTIONAL RESERVOIRS}

This section presents a brief summary of the main concepts and fundamentals related to rate transient analysis methods (RTA), which are classified as advanced analytical techniques to evaluate reservoir production performance-i.e., estimation of reservoir properties, original volumes in place, and ultimate recovery. In essence, RTA methods are analogues to pressure transient (PTA) methods, with the difference that RTA is centered on the declining production rates and not on pressure data.

The approach of this work is mainly focused on providing a better understanding of the RTA methods commonly used to analyze production data from multi-fractured horizontal wells (MFHW), since this is the most common completion method in the modern unconventional plays. As an introduction to the RTA concepts, Table 2 shows a comparison of DCA and RTA methods to understand their main differences.

In general, RTA techniques are grounded on the same principles of the fluid flow in porous media theory-i.e., diffusivity equation-just like PTA techniques. However, there are several different RTA techniques and each technique has different additional assumptions, considerations and restrictions that go into the model itself. For this reason, it is advised that interpreters understand the principles and the special features of each method to avoid misapplications and misleading results.

Initially, RTA methods were designed for conventional reservoirs (vertical wells mainly), but recently such interpretation techniques have evolved to provide better solution to the fluid flow and storage complexities of the new unconventional wells-i.e., naturally occurring fractures, multiple lithology, multiple fractures in long horizontal wells, multiphase flow, adsorption capacity, non-Darcy flow, and non-static (stress dependent) permeability. One of 
the most important advantages of the RTA techniques over the DCA models is the capability to account for these special attributes is. Consequently, the RTA methods are suited to estimate reservoir and completion (fracture network) characteristics-from transient flow data-and to forecast ultimate recovery (EUR) and original volumes-in-place (OGIP/OOIP)-from boundarydominated flow data-even under changing operational conditions (Clarkson, 2013b). However, as pointed out by Anderson, D.M. et al. (2012), the same reservoir complexities of the unconventional plays lead to non-unique solutions of reservoir characteristics and production forecasts, which is a big challenge for RTA techniques.

Table 2-Comparison of DCA and RTA techniques.

\begin{tabular}{|c|c|c|}
\hline Features & DCA & RTA \\
\hline Main Input Data & Production rates & Production rates and flowing pressures \\
\hline Additional Required Data & & $\begin{array}{c}\text { Fluid and reservoir properties, wellbore } \\
\text { configuration* and number of fracture stages } \\
\text { (MFHW) }\end{array}$ \\
\hline Fundamentals & Empirical & Fluid flow physics \\
\hline Methodology & $\begin{array}{l}\text { Historical production decline trends are } \\
\text { fitted by rate-time models }\end{array}$ & $\begin{array}{c}\text { Formation properties characterization and } \\
\text { simulation of the reservoir production } \\
\text { performance according to specific analytical } \\
\text { models }\end{array}$ \\
\hline Deliverables & $\begin{array}{l}\text { Production forecast and prediction of } \\
\text { recoverable reserves under current } \\
\text { flowing conditions }\end{array}$ & $\begin{array}{c}\text { Original volumes in-place, production forecast, } \\
\text { estimation of recoverable reserves under } \\
\text { variable flowing conditions, reservoir } \\
\text { properties (e.g., K and S), completion features } \\
\text { (e.g., Xf, kf), drainage area, estimation of } \\
\text { reservoir pressure }\end{array}$ \\
\hline
\end{tabular}

RTA techniques include a wide range of methods that can be divided into the following categories:

- Specialized plots (straight line analysis). 
- Type-curve analysis.

- Flowing material balance.

- Analytical models.

- Numerical models.

Due to the high complexity associated to unconventional reservoirs, it is recommended to combine several RTA techniques to find the best possible solution, particularly when analyzing MFHW exhibiting a wide range of flow regime signatures (Clarkson and Pedersen, 2010). Additionally, diagnostic analysis should be carefully performed and be based not only on the production performance fundamentals, but also on the analyst's knowledge and experience of each case. A summary of RTA techniques will be presented in this section, giving special importance to those designed to provide solution to MFHW in unconventional reservoirs.

\subsection{Superposition Time Functions and Gas Flow Considerations}

\subsubsection{Superposition Time Functions}

Superposition functions are grounded on the principle that the final response of a system submitted to several rate changes is equal to the sum of the individual response of each rate change-i.e., multiple single drawdowns starting at different times (Agnia et al., 2012). This principle means that superposition time functions (STF) have an improved analysis capacity for production data from wells flowing under unstable operating conditions, such as stepchanges and fluctuations of flowing pressures. However, these time functions transform the data, so that its flow regime signature in the diagnostic plots tends to look like the flow regime for which they have been created (Clarkson, 2013b). Thus, analyzing wells submitted to

multiple and frequent operational changes-where a high degree of discontinuities is expected-tends to make this effect more prominent and increases the risk of a possible misinterpretation of flow regimes (Anderson and Mattar, 2003). According to Agnia et al. (2012), superposition-time functions can be biased and misleading in many cases due to multiple factors such as well cleanup, outliers, liquid loading, high scatter and interference. As a 
result, superposition functions may generate erroneous straight-line trends in the diagnostic and analysis plots, which can be misinterpreted as unrealistic flow regimes and erroneous reservoir properties may be estimated. They have proposed a workflow to check if bias on superposition-time specialized plots exists and to avoid those mistakes by filtering the input data. One of the main bias sources is the flow rate instability, centered on two key effects, the noise and outliers. While noise is related to the scattered points that are slightly deviated from the main central production decline trend, outliers refer to those points placed away from the average production decline trend. Some causes of those worthless points are rate averaging, liquid loading, variable operating conditions, production interference, and measurement and allocation problems.

Table 3-Superposition time functions for different flow regimes (Agnia et al.,
\begin{tabular}{|c|c|c|}
\hline Flow regime & Nom & Superposition Time Functions \\
\hline Radial & Super-log(t) & $\sum_{i=1}^{n} \frac{q_{i}-q_{i-1}}{q_{n}} \log \left(t_{i}-t_{i-1}\right.$ ) \\
\hline Linear & Super- $\sqrt{t}$ & $\sum_{i=1}^{n} \frac{q_{i}-q_{i-1}}{q_{n}} \sqrt{t_{i}-t_{i-1}}$ \\
\hline Bilinear & Super- $\sqrt[4]{t}$ & $\sum_{i=1}^{n} \frac{q_{i}-q_{i-1}}{q_{n}} \sqrt[4]{t_{i}-t_{i-1}}$ \\
\hline Spherical & Super- $\sqrt[2]{t}$ & $\sum_{i=1}^{n} \frac{q_{i}-q_{i-1}}{q_{n}} \frac{1}{\sqrt{t_{i}-t_{i-1}}}$ \\
\hline Pseudo-Steady State & Super-t & $\sum_{i=1}^{n} \frac{q_{i}-q_{i-1}}{q_{n}}\left(t_{i}-t_{i-1}\right)$ \\
\hline Boundary dominated & $t_{M B}$ & $\frac{G_{p_{n}}}{q_{n}}$ \\
\hline
\end{tabular}

The general form of the superposition time functions $\left(t_{\mathrm{SP}}\right)$ is presented in Eq. 33. The specific time function $\left[f\left(t_{n}\right)\right]$ depends on the flow regime, as presented in Table 3. 


$$
t_{S P}=\sum_{j=1}^{n} \frac{\left(q_{j}-q_{j-1}\right)}{q_{n}} f\left(t_{n}-t_{n-1}\right)
$$

In theory, the ideal STF should be a compound function with the ability of changing between the single functions depending on the happening flow regime. However, in reality, it is not possible (Liang et al., 2012). In the case of UCR, boundary-dominated and linear are the two most common superposition functions because those are the prevalent flow regimes in these kinds of ultra-low permeability reservoirs. Therefore, the two most important superposition functions are the linear superposition and the material balance time function (MBT or $\mathrm{t}_{\mathrm{MB}}$ ).

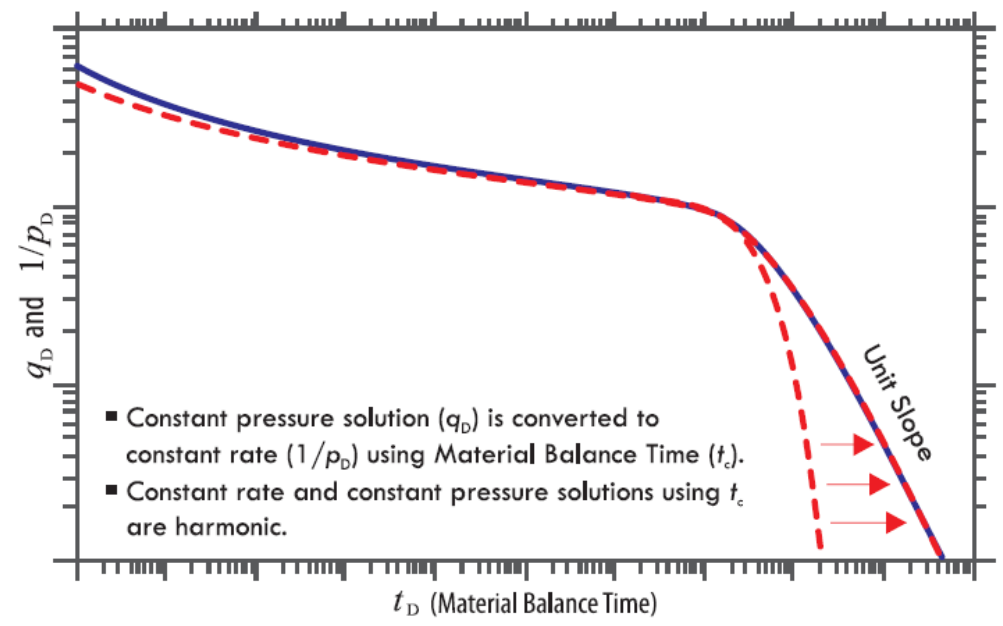

Fig. 8-Material Balance Time plot showing the equivalency of qD and 1/pD (Fekete, 2013a)

The Material Balance Time function (MBT), developed by Blasingame et al. (1991), effectively converts the constant pressure solutions into the equivalent constant rate solutions during BDF regime and provides a good approximation in case of linear flow. For production analysis purposes, it is a great advantage since most of the fluid flow solutions have been developed in the well testing field assuming constant flow rate (Anderson and Mattar, 2003). Fig. 8 illustrates the fundamentals of the MBT and how the constant pressure solution (which is 
originally exponential) is converted to constant rate (which is harmonic). According to Liang et al. (2012), although the material balance time was created for BDF conditions and should only be used when all the drainage area boundaries have been reached, it is also recommended as the best superposition time to analyze variable production data in linear flow regime. A study developed by Anderson and Mattar (2003) has also shown that when material balance time is used in diagnostic plots to identify flow regimes and estimate reservoir properties, it provides reliable results, independent of the flow regime. Fig. 9 illustrates the practical concept of material balance time. Note that MBT is frequently represented by the symbol $t_{c}$ also, which means corrected time based on cumulative production (Fekete, 2013b).

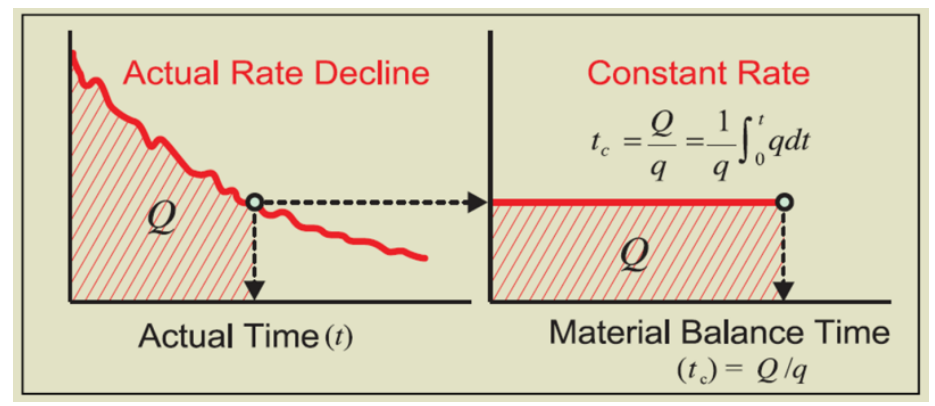

Fig. 9-Illustration of the Material Balance Time concept (Fekete, 2013a)

\subsubsection{Pseudovariables}

The main purpose of pseudovariables is to account for the variation of some gas properties with pressure (Z-factor, compressibility and viscosity), in such a way that the analytical solutions for fluid flow-which assume single phase flow of a slightly-compressible or non-compressible fluid-can be applied to gas wells (Clarkson, 2013a). The next three equations correspond to the pseudopressure (Eq. 34), pseudotime (Eq. 35) and material balance pseudotime (Eq. 36). Note that the pseudopressure correlation accounts for changes in viscosity and Z-factor, while pseudotime and material balance pseudotime corrects for the 
pressure-dependency of gas compressibility and viscosity, both of which are evaluated at average reservoir pressure conditions.

$$
\begin{aligned}
& \psi_{i}-\psi_{w f}=\int_{P w f}^{P i} \frac{P}{\mu_{g} Z} d p \\
& t_{a}=\left(\mu_{g} C_{t}\right)_{i} \int_{0}^{t} \frac{d t}{\bar{\mu}_{g} \bar{C}_{t}} \\
& t_{c a}=\frac{\left(\mu_{g} C_{t}\right)_{i}}{q_{g}} \int_{0}^{t} \frac{q_{g} d t}{\overline{\mu_{g}} \overline{C_{t}}}
\end{aligned}
$$

\subsection{Specialized Plots (straight-line analysis)}

Specialized plots, also known as straight-line methods, offer two important applications: flow regime identification and reservoir properties characterization. Flow regimes identification is a critical and important task in the production analysis, because the following steps depend on the proper diagnosis of the flow regimes. Thus, a mistake in this step will affect the entire analysis. Once flow regimes have been identified, specific plots are used to linearize the dataset corresponding to each flow geometry, and depending on each case, reservoir properties and fracture network features may be derived (Clarkson, 2013b). Therefore, specialized plots are considered as a valuable tool and a compulsory technique when analyzing production performance.

\subsubsection{Flow Regimes in Multi-Fractured Horizontal Wells}

Several flow regimes may be developed by multi-fractured horizontal wells in unconventional plays as a product of the multiple parameters in the complex reservoir/fractures system (Clarkson, 2013a; Clarkson and Pedersen, 2010; Liang et al., 2012; Luo et al., 2010; Mattar and Anderson, 2003). The ideal flow regimes sequence is described below. Generally, most of these flow regimes are not developed or its duration is insignificant. Therefore, only the dominant flow regime are exhibited and easily identified. 


\subsubsection{Bilinear Flow}

Initial production is dominated by the high conductivity induced fracture network. However, due to the fracture's low storage capacity, its effect is short-sometimes, impossible to observe-and declines quickly. On the contrary, the low permeability matrix develops long and stable transient flow periods (Anderson et al., 2010). The fracture transient flow signature and its performance depend on the type of fracture. Three models have been proposed (Ahmed, 2010). Infinite-conductivity vertical fractures, finite-conductivity vertical fractures and uniform-flux vertical fractures. The two most common models are described as follows:

- Infinite-conductivity vertical fractures: Characterized by very high conductivity (considered infinite for practical applications) and no pressure loss along the fracture extension. This kind of fractures exhibits a linear flow period, followed by formation flow regimes. This type of fracture is representative of highly propped fractured wells.

- Finite-conductivity vertical fractures: Characterized by lower fracture conductivity in comparison with the infinite-conductivity fractures and the consequent pressure drop along the fracture extension (from fracture tip to wellbore). These fractures usually result from massive hydraulic fracture operations where fractures are not very well propped, so it is considered the distinguishing type of fracture of the MFHW in UCR. This kind of fractures initially exhibits a very short linear flow period (flow inside the fracture), followed by a bilinear flow period (linear flow inside the fracture and the linear flow from matrix to fracture). The first linear flow period is so insignificant that it is ignored. Thus, the bilinear flow period is considered as the first flow signature of the MFHW, although in most cases it is rarely observed. 


\subsubsection{Early Linear Flow}

Also known as "primary formation linear flow", early linear flow is caused by dominant linear flow from formation towards fractures. Flow direction is perpendicular to fracture planes and each fracture behaves independently.

\subsubsection{Early Radial Flow}

The flow transient effect continues growing perpendicular to the fracture plane, but it also extends out of the area stimulated by the fracture network. Therefore, a radial flow profile is generated, although it could be more elliptical depending on the fracture network geometry.

\subsubsection{Early Transition Flow}

This flow period, also known as "fracture interference", corresponds to the transition between the early (linear/radial) and the late (compound) linear flow regimes. At that time, the drainage area of each fracture has extended in such a way, that they start to interfere with each other.

\subsubsection{Late Linear Flow}

Also known as "compound formation linear flow", late linear flow occurs after the fractures' transient effects have interfered as wells the drainage area has extended beyond the stimulated region, and the main contribution to flow comes from the outer matrix region. The flow direction is parallel to fracture planes.

\subsubsection{Late Transition Flow}

Late transition flow corresponds to the final transition between the transient flow and the late radial flow, in case of a closed reservoir system. 


\subsubsection{Late Radial Flow}

At this stage, depending on the matrix properties, fluid flow out of the stimulated region may not be completely parallel to fracture planes. Instead, it extends out of the area limited by the horizontal well section forming a radial or an elliptical flow pattern. As the drainage area growths, the well and the fractures behave like a unique system and its flow signature becomes less important, while the outermost matrix properties become more dominant.

\subsubsection{Boundary-Dominated Flow}

Also known as the depletion state, the boundary-dominated flow regime is the final period occurring when the flow effect has finally reached the reservoir boundaries and all the drainage pore volume has been connected. Ultimate reserves of fluids-in-place can only be estimated with confidence if this condition is reached (Mattar and Anderson, 2003).

Depending on the reservoir features and the fracture network geometry, some flow regimes may or may not be exhibited, and even if present, some may not be easily observed. Fig. 10 shows a practical illustration of a flow sequence in a MFHW, including some of the previously mentioned flow regimes. According to Clarkson (2013a), it is possible to have multiple linear flow periods (e.g., early and late linear, early and late radial). Note that due to the very low permeability of the UCR, the linear transient flow regimes-i.e., early and late linear flows-dominate the reservoir production performance for a long time and are considered as the most representative of this kind of reservoirs. BDF is also observed in unconventional reservoirs during their late productive life, while bilinear and radial flows are less commonly observed during early production times (Anderson et al., 2010).

Several diagnostic methods-i.e., log-log, square root of time, fourth root of time, and linear and radial derivative-are available to identify the different flow regimes that may develop in MFHW, including transient (bilinear, linear, elliptical, and radial), transitional, and boundary-dominated flows. 


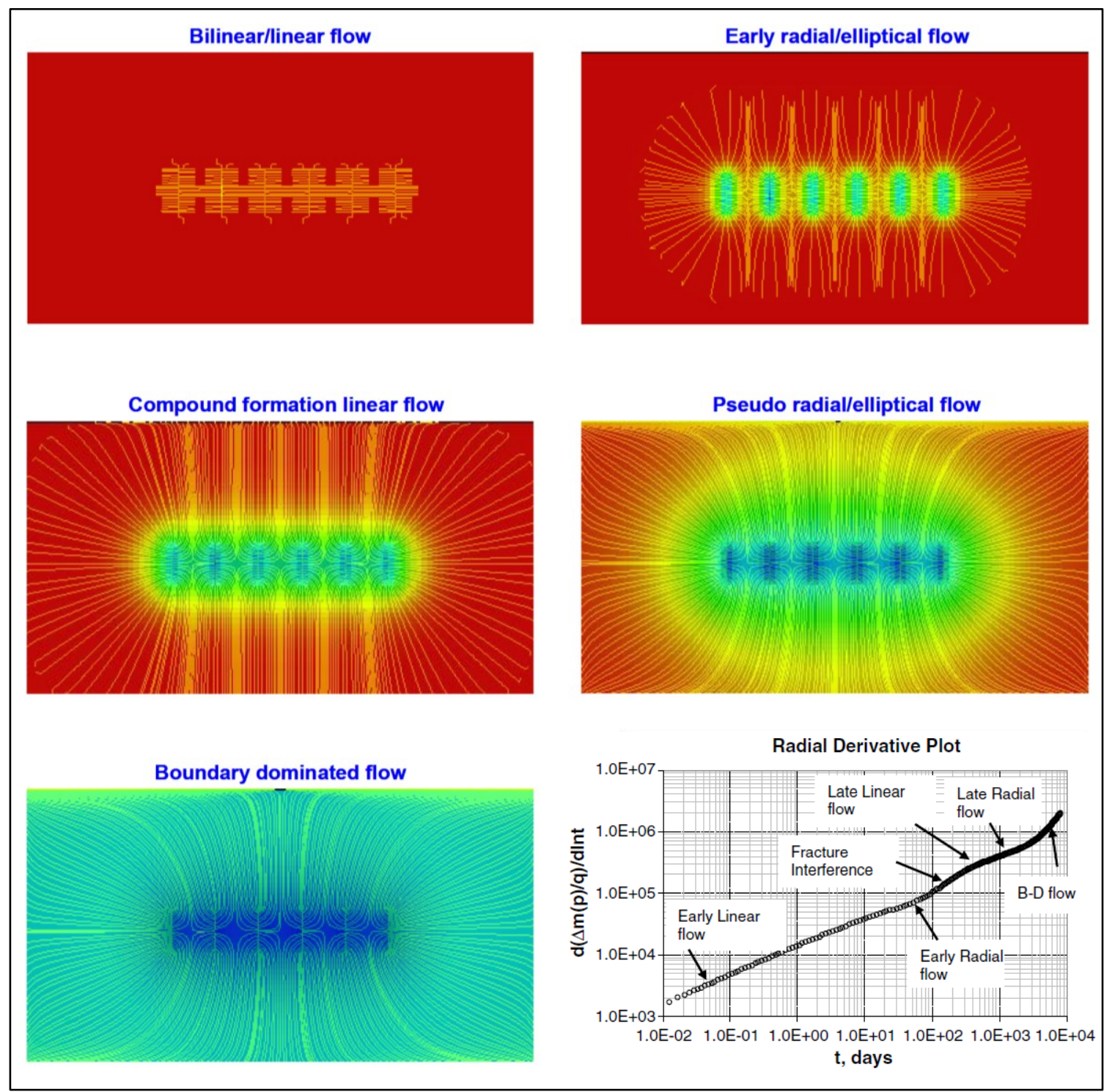

Fig. 10-Example of flow regimes in a MFHW generated by streamlines simulation (Luo et al., 2010) and flow regime signatures in a radial derivative plot (Clarkson, 2013a)

Flow regime signatures appear not only in the previously mentioned diagnostic plots, but in most of the analysis plots. For example, in the Flowing Material Balance plot, the BDF is exhibited as a straight line. Although the main purpose of analysis plots is not to diagnose flow regimes, they can be used to verify the proper identification and selection of the flow regimes during the diagnostic work. 
This study is not intended to provide a detailed guideline of how to apply diagnostic plots, but to present a summary of the main diagnostic plots presented in literature and those commonly used by interpreters, including its advantages and some practical applications. Production performance is affected by multiple production mechanisms, as well as by a combination of complex reservoir properties and unique completion features. Therefore, each case is different and there is not a simple diagnostic workflow to investigate all the possible outcomes. Following, the superposition-time-functions concept will be explained and later, the most important Log-Log and Cartesian straight line methods to analyze MHFW will be described, including plotting parameters (axis variables), estimated reservoir/fracture properties, pseudotime corrections and flow regime diagnostic concepts (slopes).

\subsubsection{Log-Log Plots}

Basically, the Log-Log plot is used to identify flow regimes and it is very useful when there is noisy data, since scattered data tends to be amplified in the derivative plots (Clarkson, 2013a). Different Log-Log plots are frequently used by interpreters, depending on the type of fluid and on the flowing conditions. Those plots can be grouped on two sets according to the selected dependent variable (x-axis): Pressure-Normalized Rates or Rate Normalized Pressures. As pointed out by Clarkson (2013a), normalized variables (PNR and RNP) and superposition functions are used to account for variable flowing conditions (See Section 3.1.1), while pseudovariables, such as pseudotime $\left(t_{c a}\right)$ and pseudopressure $(\Psi)$, are used to better represent the changing pressure-dependent gas properties (See Section 3.1.2).

Note that if pressure data is absent, the Log-Log analysis may be performed only with rate data (y-axis) to have an approximate approach of the flow regimes. In the same way, when analyzing gas wells, if pseudo-pressure data is not available, regular pressure data may be used. However, it should be considered that, depending on the stability of the flowing conditions, the reliability of the results may be compromised. Additionally, though real time or superposition time could be used, material balance time (MBT: $t_{c}$ or $\left.t_{c a}\right)$ is recommended as the best superposition function (x-axis) to account for the variable operating conditions. In conclusion, the best practice is to use normalized variables (PNR or RNP) and MBT for oil wells, while 
pseudo-variables (pseudo-pressures) and pseudo time functions (material balance pseudotime, $t_{c a}$ ) are encouraged for gas cases, if possible (See Table 4).

Table 4-Log-Log plots for flow regime identification.

\begin{tabular}{|c|l|c|c|}
\hline \multicolumn{2}{|c|}{ Pressure Normalized Rates (PNR) } & y-axis & x-axis \\
\hline \multirow{2}{*}{ Oil } & PNR vs. Time & $\mathrm{q} / \Delta \mathrm{P}$ & $\mathrm{t}$ \\
\cline { 2 - 4 } & PNR vs. MBT & $\mathrm{q} / \Delta \mathrm{P}$ & tc \\
\hline \multirow{2}{*}{ Gas } & PNR vs. Pseudo-time & $\mathrm{q} / \Delta \mathrm{m}(\mathrm{P})$ & ta \\
\cline { 2 - 4 } & PNR vs. MBpT & $\mathrm{q} / \Delta \mathrm{m}(\mathrm{P})$ & tca \\
\hline \multirow{2}{*}{ Rate Normalized Pressures (RNP) } & $\mathbf{y}$-axis & x-axis \\
\hline \multirow{2}{*}{ Oil } & RNP vs. Time & $\Delta \mathrm{P} / \mathrm{q}$ & $\mathrm{t}$ \\
\cline { 2 - 4 } & RNP vs. MBT & $\Delta \mathrm{P} / \mathrm{q}$ & tc \\
\hline \multirow{2}{*}{ Gas } & RNP vs. Pseudo-time & $\Delta \mathrm{m}(\mathrm{P}) / \mathrm{q}$ & ta \\
\cline { 2 - 4 } & RNP vs. MBpT & $\Delta \mathrm{m}(\mathrm{P}) / \mathrm{q}$ & tca \\
\hline
\end{tabular}

Flow regimes exhibit the next signatures (slopes) in the Log-Log plots:

- Pressure-Normalized Rates:
o Bilinear Flow: $-1 / 4$
○ Linear Flow: $-1 / 2$
○ BDF: $\quad-1$

- Rate Normalized Pressures:
○ Bilinear Flow: 1/4
- Linear Flow: $1 / 2$
○ BDF: $\quad 1$

Fig. 11 shows an example of a gas well, where bilinear and linear flows were identified using the $\Psi$ NR vs. MBT plot. Note that BDF line is shown only for reference, because production 
data doesn't exhibit the -1 slope yet. According to Anderson et al. (2010), the skin damage may mask the linear signature on the Log-Log plot.

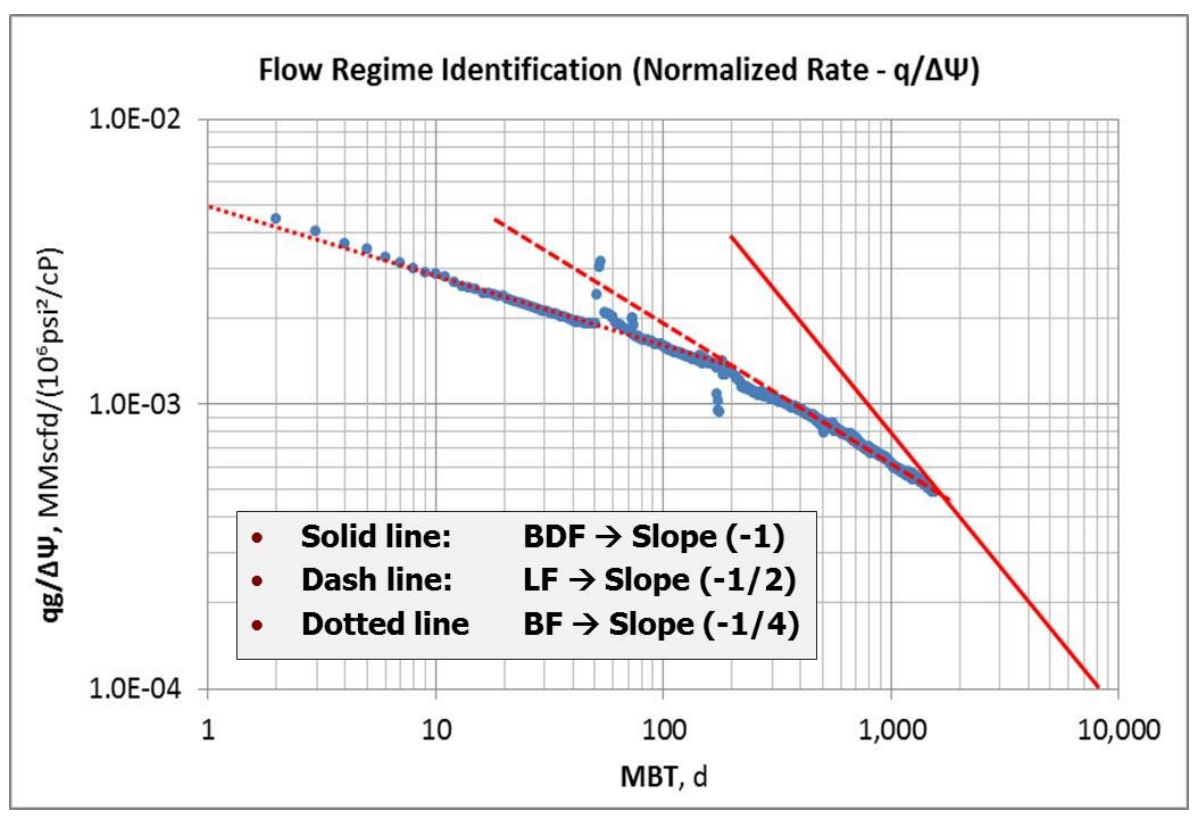

Fig. 11-Example of flow regimes identified by using a Log-Log plot.

\subsubsection{Derivative Plots}

As with the previous Log-Log technique, production data is plotted on logarithmic scales, but in this case the dependent variable (y-axis) corresponds to the radial, linear or bilinear derivative, as presented in Table 5. Note that all parameters are in real units. In case of gas wells, pressures should be replaced by pseudopressures and time by pseudotime. If flowing conditions are not stable, real time should be replaced by material balance time (oil cases) or material balance pseudotime (gas cases). However, be aware that in any case derivatives are significantly affected by noisy data, this being its main drawback. 
Table 5-Derivative plots

\begin{tabular}{|c|c|}
\hline Plot & Axis Parameters \\
\hline Radial Derivative & $\frac{\partial}{\partial \ln t}\left[\frac{P_{i}-P_{w f}}{q}\right]$ vs.t \\
\hline Linear Derivative & $\frac{\partial}{\partial \ln \sqrt{t}}\left[\frac{P_{i}-P_{w f}}{q}\right]$ vs.t \\
\hline Bilinear Derivative & $\frac{\partial}{\partial \ln \sqrt[4]{t}}\left[\frac{P_{i}-P_{w f}}{q}\right]$ vs.t \\
\hline
\end{tabular}

Flow regimes can be identified from the previous plots using the following guideline:

- Bilinear flow appears as a flat (zero) slope in the bilinear derivative and as a quarter-slope (1/4) in the radial derivative.

- Linear flow is exhibited as a clear half-slope on the radial derivative, while it appears as a flat (zero) slope in the linear derivative. As Anderson et al., (2010) mentioned, the skin effect impacts the classic straight trend of the linear flow in the SRT plot, but not in the derivative plots, which represents a considerable advantage.

- Radial flow is exhibited as a flat (zero) slope in the radial derivative.

- BDF appears as a positive unitary-slope (1) in the radial derivative plot

Fig. 12 shows a schematic representation of the different possible flow signatures exhibited by a MFHW on a radial derivative plot (Clarkson and Pedersen, 2010).

\subsubsection{Fourth Root-Time Plot (Bilinear Flow)}

Bilinear flow appears as a straight line on the fourth root-time specialized plot (Cartesian). From slope, information about the hydraulic fracture conductivity $\left(k_{f} w_{f}\right)$ can be extracted, if average reservoir permeability is known (Clarkson, 2013a). 


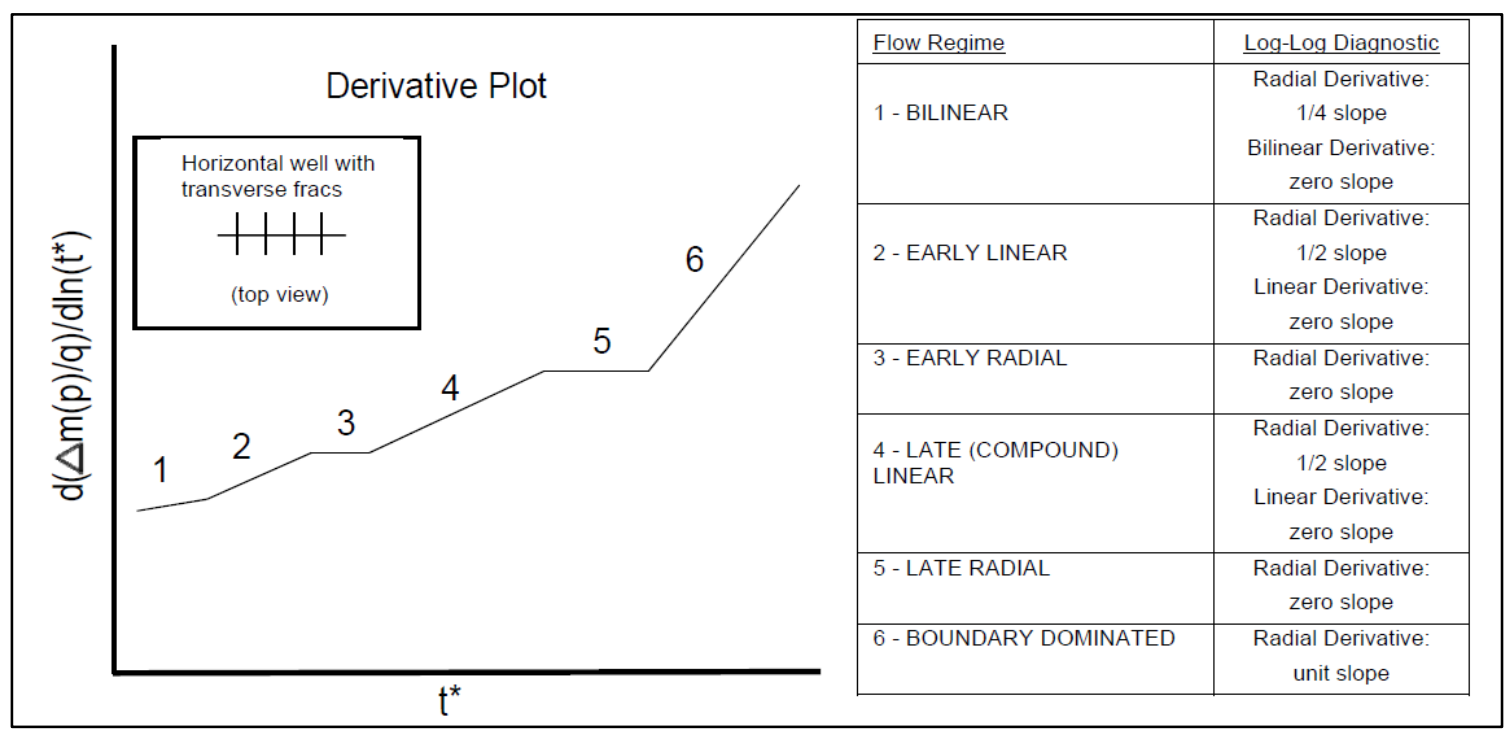

Fig. 12-Illustration of flow regime sequence for a MFHW in a single porosity reservoir (Clarkson and Pedersen,

2010).

In case of oil wells, plot $\frac{\Delta P}{q_{o}} v s . \sqrt[4]{t}$. In case of gas wells, plot $\frac{\Delta \Psi}{q_{g}} v s . \sqrt[4]{t_{a}}$. The time variable $(t)$ is real time for oil wells and pseudo-time $\left(t_{a}\right)$ for gas wells. In case of variable flowing conditions, the bilinear superposition time function ( $t_{\text {BLS }}$ ) should be used instead of $\sqrt[4]{t}$ (Eq. 37).

$$
t_{B L S}=\sum_{j=1}^{n} \frac{\left(q_{j}-q_{j-1}\right)}{q_{n}}\left(t_{n}-t_{n-1}\right)^{1 / 4}
$$

Finally, the hydraulic fracture conductivity can be estimated from the straight line slope $(\mathrm{m})$, according to the next equation (gas case):

$$
\left(w_{f} k_{f}\right)^{1 / 2}=\frac{443.2 T}{\sqrt[4]{k} m h \sqrt[4]{\emptyset \mu_{g i} c_{t i}}}
$$

\subsubsection{Square Root-Time Plot (Linear Flow)}

This is considered one of the most important diagnostic plots, since linear flow resulting from the long transient matrix drainage into the fractures is the dominant flow regime 
in most of the unconventional reservoirs (Anderson et al., 2010). In addition, valuable information of the reservoir and the fracture network may be extracted from its analysis. Basically, the fracture network features are expressed by three variables (Rasdi and Chu, 2012), slope of the formation linear flow straight line, time of end linear flow, and the Y-axis intercept.

Linear flow appears as a straight line on the squared root-time (SRT) specialized plot in Cartesian coordinates. The fracture half-length $\left(\mathrm{x}_{\mathrm{f}}\right)$ can be estimated if average reservoir permeability is known. In case of oil wells, $\frac{\Delta P}{q_{o}} v s . \sqrt{\mathrm{t}}$ is plotted, while $\frac{\Delta \Psi}{q_{g}} v s \cdot \sqrt{\mathrm{t}_{a}}$ is plotted in case of gas wells. If variable flowing conditions are presented, the linear superposition time function ( $t_{L S}$ ) should be used instead of $\sqrt{t}$ (Eq. 39). In any case, the time variable (t) is real time for oil wells and pseudo-time $\left(t_{a}\right)$ for gas wells.

$$
t_{L S}=\sum_{j=1}^{n} \frac{\left(q_{j}-q_{j-1}\right)}{q_{n}}\left(t_{n}-t_{n-1}\right)^{1 / 2}
$$

Finally, the fracture half-length can be estimated from the straight line slope $(\mathrm{m})$, according to Eq. 40 (gas case):

$$
x_{f}=\frac{315.4 T}{\sqrt{k} m h \sqrt{\varnothing \mu_{g i} c_{t i}}}
$$

Additionally, OGIP can be also estimated from the SRT plot by detecting the end of the linear flow, which appears as a deviation from the straight line. Clarkson (2013a); Nobakht and Clarkson (2012b); Wattenbarger et al. (1998) provide a detailed description of such calculations. Fig. 13 shows an example of the SRT plot, showing the end of the linear flow (the green dotted line represents the end of the linear flow and the beginning of the BDF). 


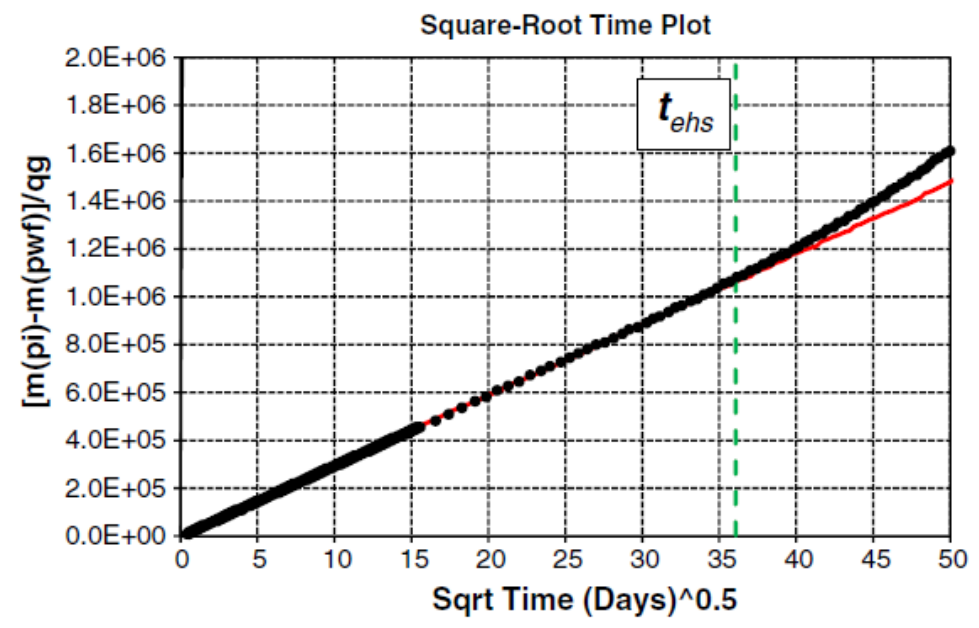

Fig. 13-Example of the squared root time plot (Clarkson, 2013a)

Most of the time, a skin effect is observed in fractured unconventional wells during the linear flow period due to the flow convergence or pressure loss along the finite conductivity hydraulic fractures. This apparent skin effect $\left(S^{\prime}\right)$-different from the mechanical skin damage in the wellbore sandface-corresponds to a constant pressure loss that can be identified on the square root-time plot as the y-intercept $b^{\prime}$. It can be calculated using the following equation (Anderson et al., 2010)

$$
S^{\prime}=\frac{k h}{1417 T} b^{\prime}
$$

Production analysts must be aware of the masking effect of the apparent skin generated by the finite conductivity of the fracture network because it may affects the primary linear flow pattern of the production data. Anderson, D.M. et al. (2012) suggest using the semilog derivative to avoid the skin impact.

Another linear flow diagnostic method is the "Linear Flow Plot", which is similar to the SRT plot, but linear superposition time (or linear superposition pseudotime in case of gas wells) 
is used instead of real time. According to (Clarkson, 2013a) superposition time is advised when analyzing linear flow data with variable flowing conditions.

\subsubsection{Specialized Plots Summary}

Table 6, presented by Clarkson and Pedersen (2010), provides a summary of the main straight line methods used to analyze MFHW, including plotting parameters (axis variables), estimated reservoir/fracture properties, pseudo-time corrections and flow regime diagnostic concepts (slopes). Note that besides the previously discussed specialized plots, other flow regimes (i.e., elliptical and pseudo-radial) are included in this summary, despite the fact these flow regimes are not frequently observed in unconventional reservoirs.

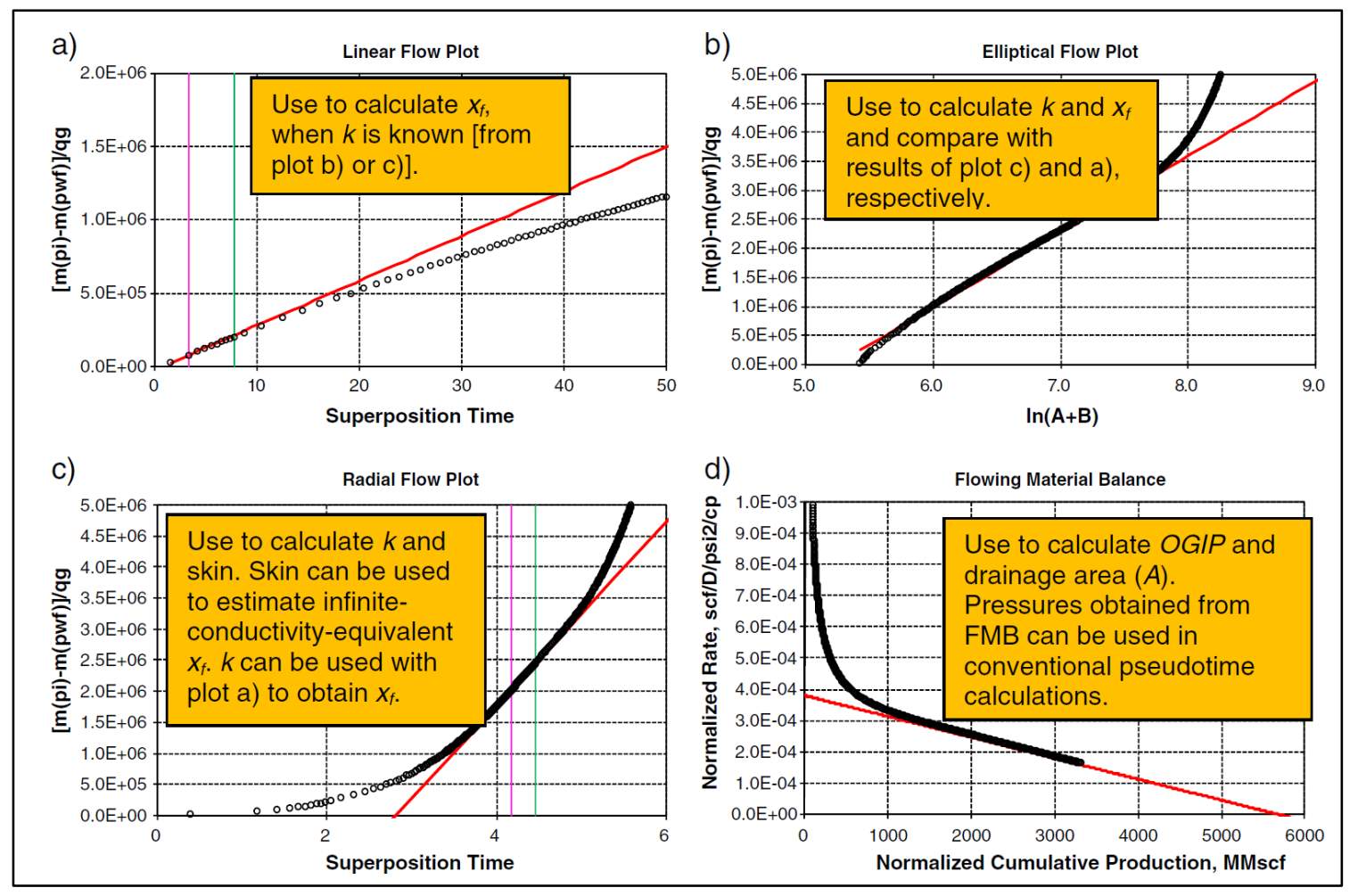

Fig. 14-Example of straight-line specialized plots (a: linear, b: elliptical, c: radial, d: BDF) 
Fig. 14 (Clarkson, 2013a) shows an example of some of the most common specialized plots to analyze flow-patterns in MFHW (identification of flow regimes and estimation of reservoir properties and features).

Table 6-Summary of specialized plots for straight-line analysis (Clarkson and Pedersen, 2010).

\begin{tabular}{|c|c|c|c|}
\hline Flow Regime & $\underline{\text { Log-Log Diagnostic }}$ & Plot & Extracted Properties \\
\hline BILINEAR & $\begin{array}{c}\text { Radial Derivative: } \\
1 / 4 \text { slope } \\
\text { Bilinear Derivative: } \\
\text { zero slope }\end{array}$ & $\begin{array}{c}\frac{\left[m\left(p_{i}\right)-m\left(p_{w f}\right)\right]}{q_{g}} \mathrm{vs} . \\
\left(t^{*}\right)^{1 / 4} \text { or } \\
\sum_{j=1}^{n} \frac{\left(q_{j}-q_{j-1}\right)}{q_{n}}\left(t_{n}^{*}-t_{j-1}^{*}\right)^{1 / 4}\end{array}$ & $\left(w_{f} k_{f}\right)^{1 / 2}(k)^{1 / 4}=\frac{443.2 T}{m h\left(\phi \mu_{g i} c_{t i}\right)^{1 / 4}}$ \\
\hline LINEAR & $\begin{array}{l}\text { Radial Derivative: } \\
1 / 2 \text { slope } \\
\text { Linear Derivative: } \\
\text { zero slope }\end{array}$ & $\begin{array}{c}\frac{\left[m\left(p_{i}\right)-m\left(p_{w f}\right)\right]}{q_{g}} \text { vs. } \\
\left(t^{*}\right)^{1 / 2} \text { or } \\
\sum_{j=1}^{n} \frac{\left(q_{j}-q_{j-1}\right)}{q_{n}}\left(t_{n}^{*}-t_{j-1}^{*}\right)^{1 / 2}\end{array}$ & $\sqrt{k} x_{f}=\frac{40.93 T}{m h\left(\phi \mu_{g i} c_{t i}\right)^{1 / 2}}$ \\
\hline ELLIPTICAL & $\begin{array}{l}\text { Radial Derivative: } \\
\text { variable slope }\end{array}$ & $\begin{array}{c}\frac{\left[m\left(p_{i}\right)-m\left(p_{w f}\right)\right]}{q_{g}} \text { vs. } \\
\ln (A+B)\end{array}$ & $\begin{array}{c}k=\frac{1422 T}{m h} \\
x_{f}=\exp \left(b^{\prime}\right)\end{array}$ \\
\hline PSEUDORADIAL & $\begin{array}{l}\text { Radial Derivative: } \\
\text { zero slope }\end{array}$ & $\begin{array}{c}\frac{\left[m\left(p_{i}\right)-m\left(p_{w f}\right)\right]}{q_{g}} \text { vs. } \\
\log \left(t^{*}\right) \text { or } \\
\sum_{j=1}^{n} \frac{\left(q_{j}-q_{j-1}\right)}{q_{n}} \log \left(t_{n}^{*}-t_{j-1}^{*}\right)\end{array}$ & $\begin{array}{c}k=\frac{1637 T}{m h} \\
s=1.1513\left(\frac{b^{\prime}}{m}-\log \left[\frac{k}{\phi \mu_{g i} c_{t i} r_{w}^{2}}\right]+3.23\right)\end{array}$ \\
\hline BOUNDARY-DOMINATED & $\begin{array}{c}\text { Radial Derivative: } \\
\text { unit slope }\end{array}$ & $\begin{array}{l}\frac{q_{g}}{m\left(p_{i}\right)-m\left(p_{w f}\right)} \mathrm{vs} \\
G_{i} \frac{m\left(p_{i}\right)-m\left(p_{R}\right)}{m\left(p_{i}\right)-m\left(p_{w f}\right)}\end{array}$ & $G_{i}$ from x-intercept \\
\hline
\end{tabular}

Fig. 15 (Clarkson, 2013a) presents an example of how to identify flow regimes by applying different specialized plots, including: (a) Log-Log, (b) Transient productivity index, (c) 
Semi-log (Radial) derivative and (d) Linear derivative. As pointed out by Clarkson (2013b), linear flow exhibits a half-slope on log-log diagnostic and radial derivative plots, a $-1 / 2$ slope on transient PI plot and a zero slope on the linear derivative; while BDF appears as a flat slope on the transient $\mathrm{PI}$ plot and as an upward deviation from half-slope on all the other plots (Although, if MBT is used instead of real time, BDF would appear as an unitary slope). Finally, it is important to keep in mind that better results are obtained if multiple diagnostics are simultaneous applied.

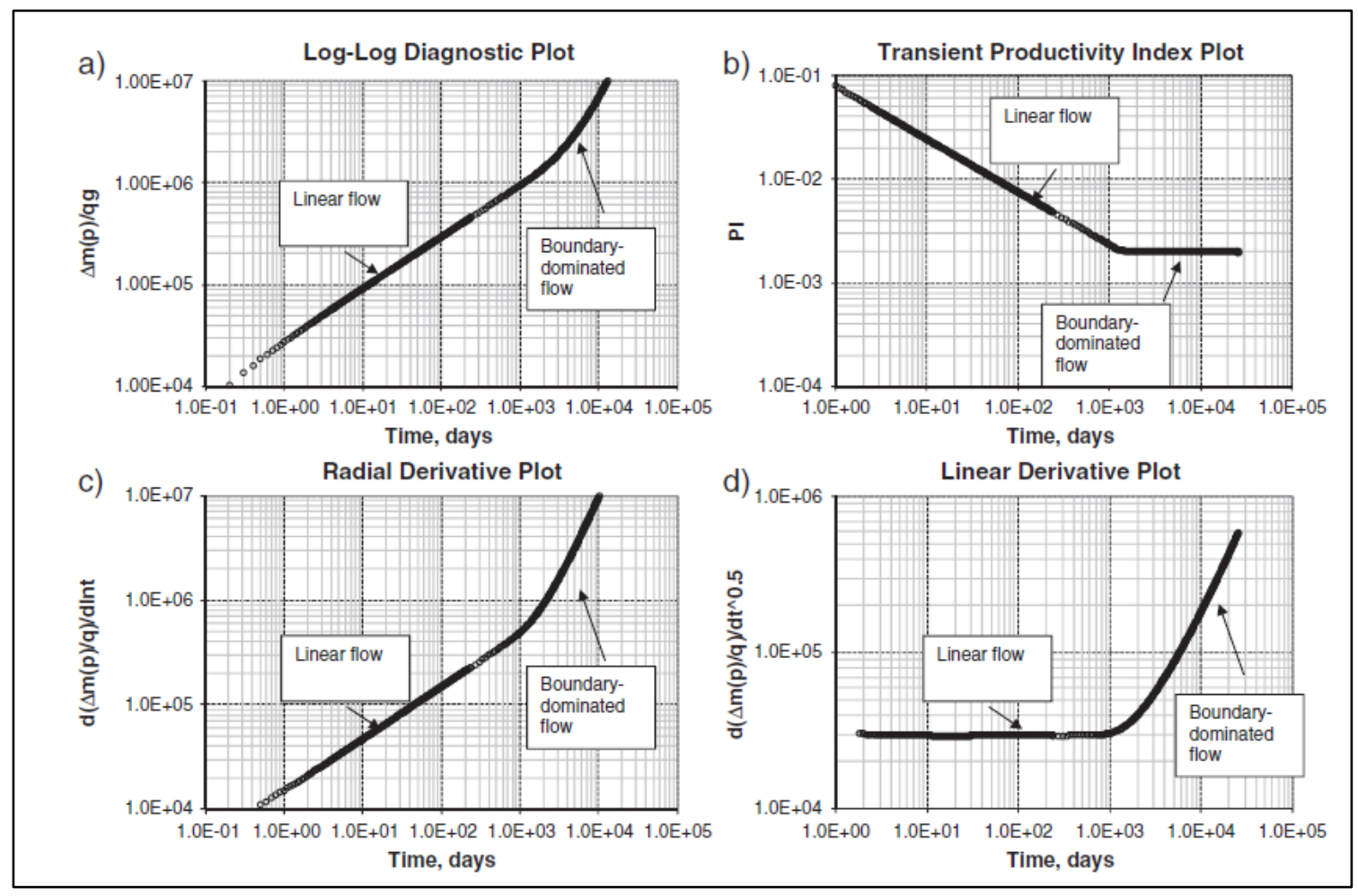

Fig. 15-Identification of flow regimes by applying different specialized plots (a: Log-Log, b: Transient productivity index, c: Radial derivative, d: Linear derivative) by Clarkson (2013b). 


\subsection{Type-curve Analysis}

This graphical procedure consists on matching real production data-expressed in dimensionless form-to dimensionless type-curves representing analytical or empirical solutions to particular reservoir models (Clarkson and Pedersen, 2010). As a result, fluids in place, reservoir and completion properties can be obtained from this procedure. Additionally, type-curves may be used to identify the dominant flow regime: Infinite Acting (radial, liner or bilinear flow), transitional (partial boundary effect) or boundary-dominated (Mattar and Anderson, 2003). EUR estimations may be obtained from typecurves analysis. However, the other production analysis techniques (i.e., the non-typecurve methods, such as DCA, specialized plots, and analytical and numerical models) should be considered as the first option to evaluate fluids-in-place, because they use Cartesian plots instead of Log-log plots. Therefore, the late time data where the BDF signal is received is not compressed by time scale (Mattar and Anderson, 2003).

\subsubsection{Fetkovich Typecurves}

In 1980 Fetkovich presented his work about decline curve analysis using typecurves. Through simple combination of material balance equations and pseudosteady-state rate equations, he demonstrated that, under specific conditions, the analytical reservoir solutions are equivalent to the empirical exponential decline model. Additionally, he provided an alternative approach when non-unique solutions are obtained from DCA. He was the first to propose typecurves for production analysis purposes-previously reserved for welltest analysis-(Mattar and Anderson, 2003) and to show that this new method could deliver a more representative solution if production mechanism is known or indicated (Fetkovich, 1980).

Fetkovich typecurves combine the early time, transient, analytical solution with the late time, pseudosteady-state empirical Arps decline curves in a single dimensionless plot. As shown in Figs. 16 through 19, the left side correspond to the analytical solution for transient flow (infinite acting period), considering an unfractured well in the center of a finite circular 
reservoir producing at a constant wellbore flowing pressure. Meanwhile, the right side is the graphical depiction of the Arps equations and represents the depletion period in boundarydominated flow. After finishing transient flow, independently of the reservoir size, all the analytical solutions for different $r_{e} / r_{w}$ ratios converge to the single exponential decline curve during BDF (Fetkovich, 1980). For this reason, the exponential decline model is considered the common solution for both flow regimes (BDF and transient). As a result, the two regions (Fig. 18) are able to represent the whole production life from transient flow during early times to boundary-dominated flow during late times (Fekete, 2013b).

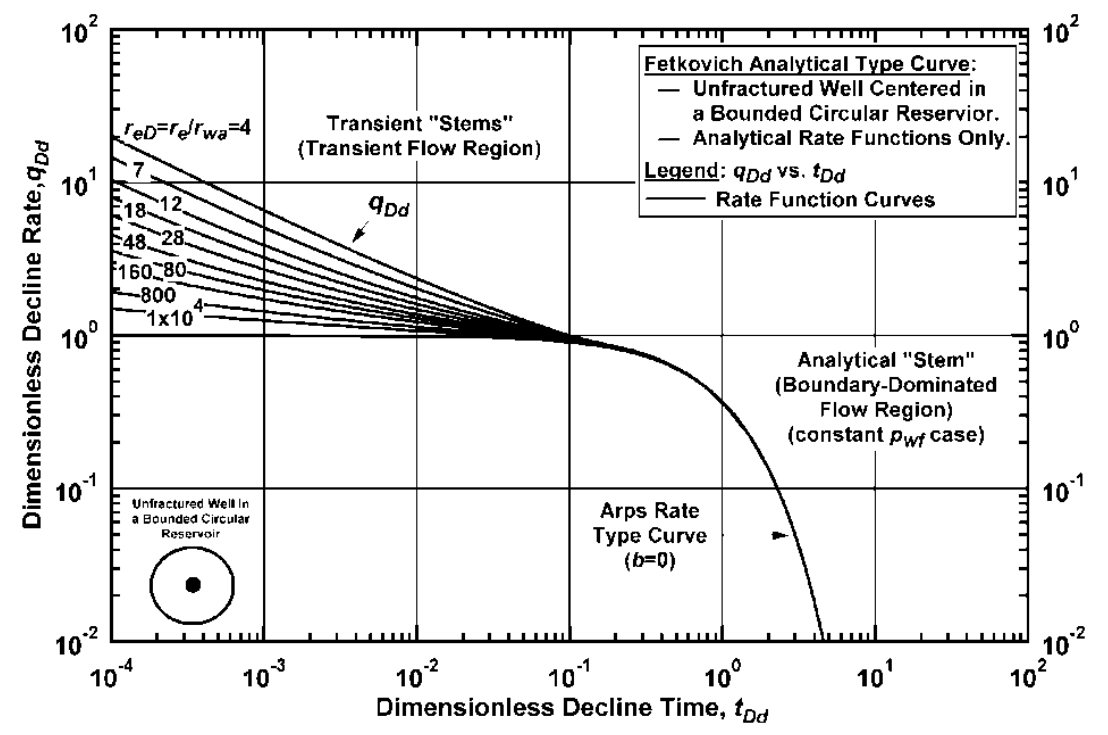

Fig. 16-Fetkovich "transient" rate type curve (Fetkovich, 1973)

Reservoir properties, such as skin, permeability and reservoir radius, can be calculated from transient data, while recoverable reserve may be estimated from depletion (BDF) data (Fetkovich et al., 1996). Even so, a problem of non-uniqueness solution exists when only earlytime data (transient region) is available. Therefore, although reservoir properties are estimated from transient data, BDF data is necessary to avoid this non-uniqueness problem. 


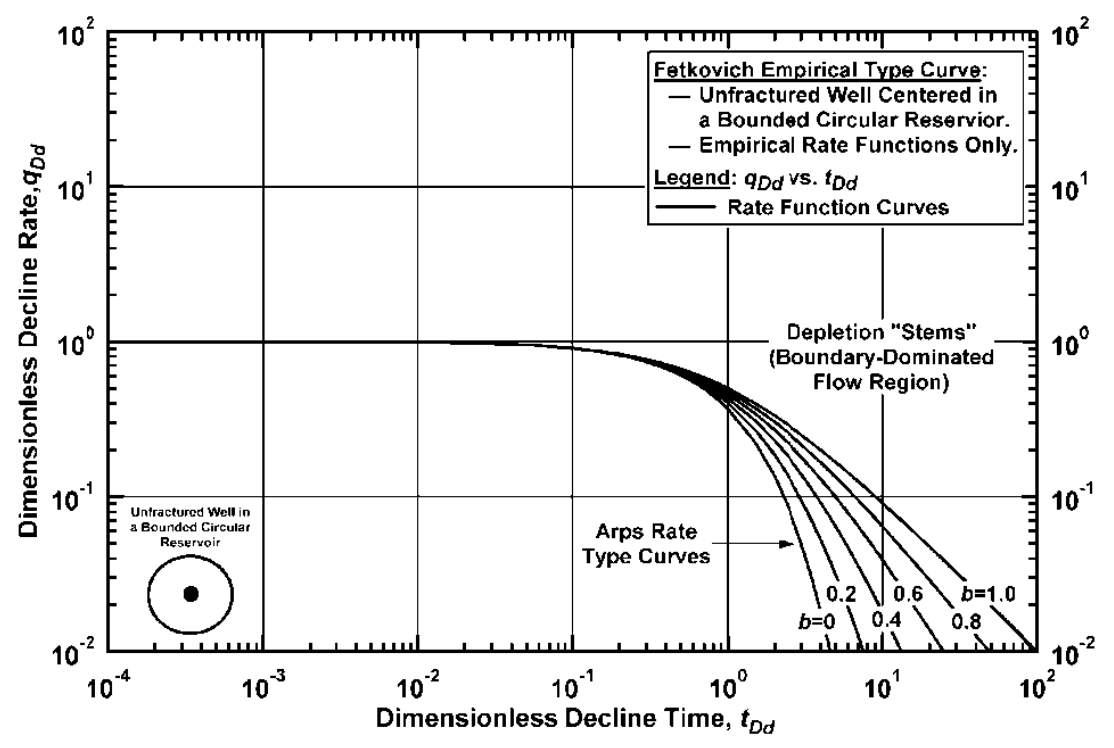

Fig. 17-Fetkovich "empirical" rate type curve (Fetkovich, 1973)

The normal range for the hyperbolic exponent " $b$ " is between 0 and 1, but Fetkovich realized that in some cases it could be higher than 1 . He explained this phenomenon as the result of forcing transient regime data to fit into the boundary-dominated region. So, forcing the production data match into the decline region (BDF) when this stabilization condition has not been reached will produce abnormal " $b$ " values, leading to the overestimation of production performance. Production performance and EUR estimations cannot be disassociated from historical operating constraints. Although Fetkovich's plots were initially proposed for stable flowing conditions-constant flowing pressures, it is possible to apply them with pressure-normalized rates, which enable this technique for a better analysis in case of unstable flowing conditions. As Fetkovich et al. (1987) mentioned: "If flowing pressures are available and are not reasonably constant but smooth and monotonically decreasing, the pressure-normalized rate, log $q / \Delta p$ vs. log $t$, should be used for analysis". 


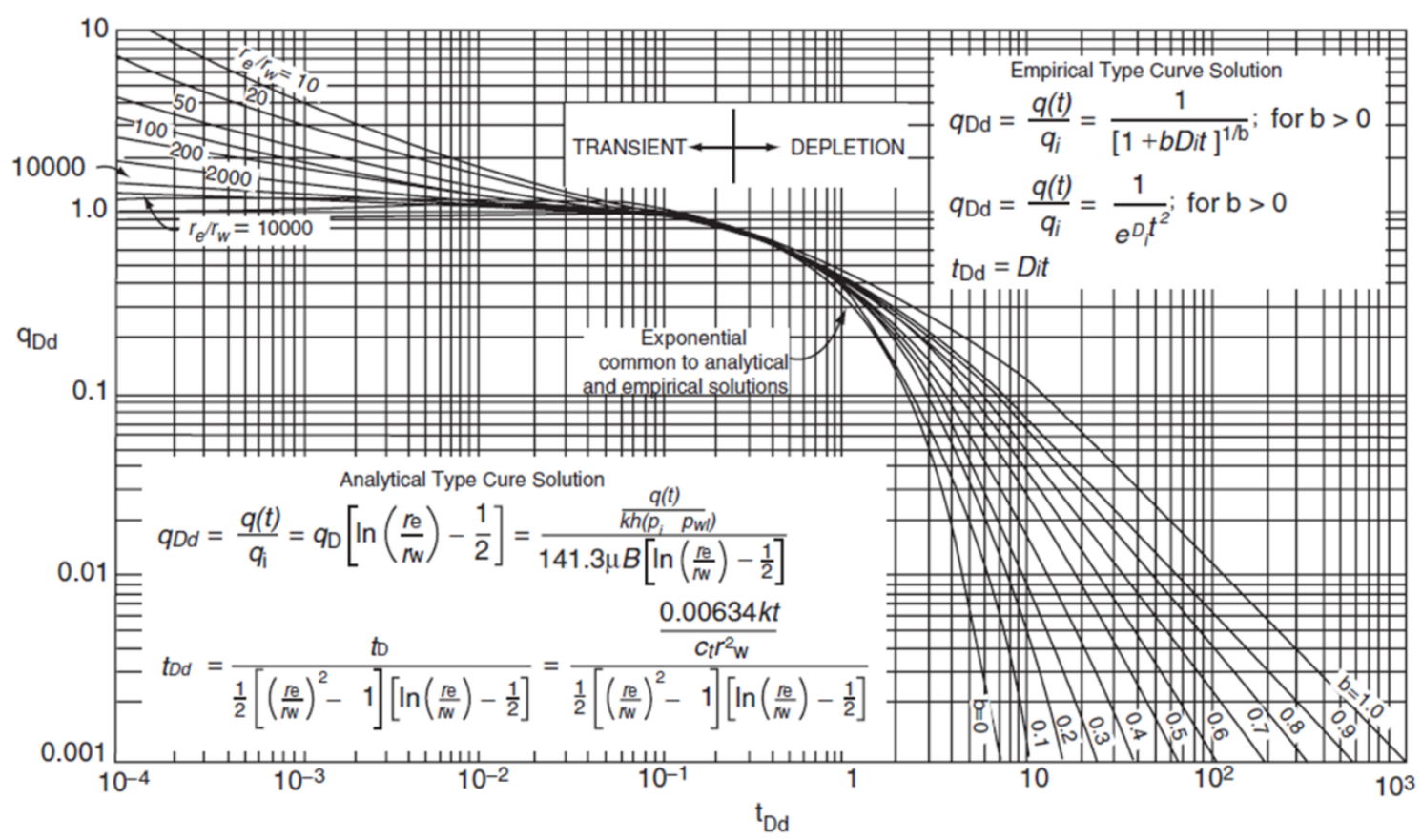

Fig. 18-Fetkovich typecurves: Composite of analytical and empirical solutions with comments (After Fetkovich, 1980).

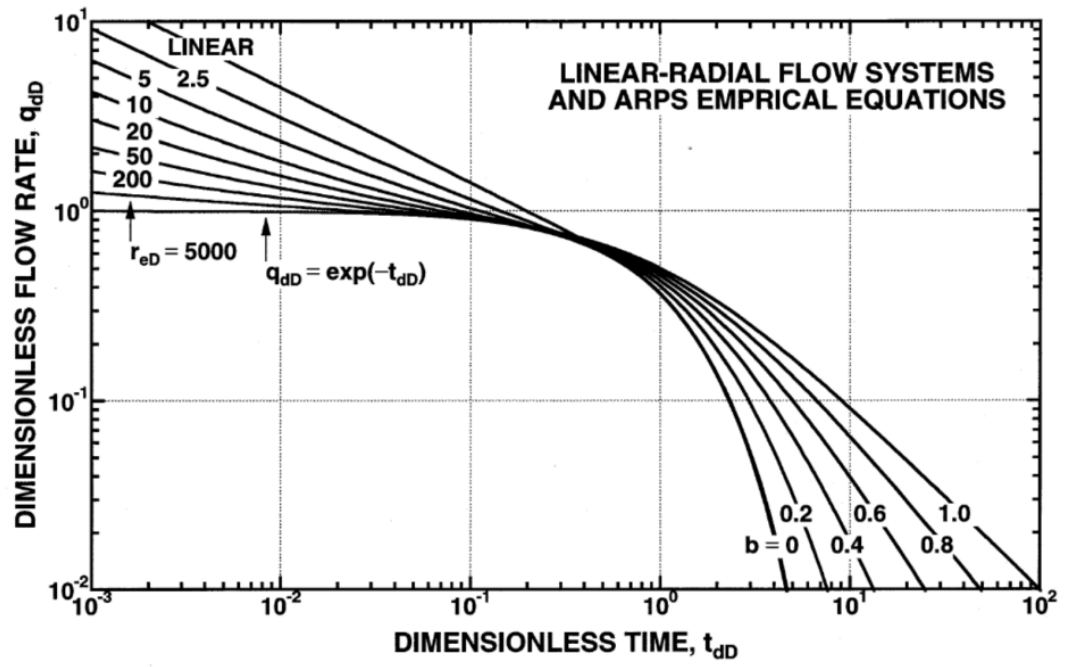

Fig. 19-Fetkovich typecurves: Composite of analytical and empirical solutions including the linear solution (Chen and Teufel, 2000). 


\subsubsection{Advanced Typecurves}

In contrast to Fetkovich typecurves, modern typecurves (e.g., Blasingame, AgarwalGardner, Wattenbarguer and Normalized Pressure Integral) use analytical solution to calculate hydrocarbons-in-place and consider the pressure-dependent properties of gas by using the pseudo-time function in the analysis (Mattar and Anderson, 2003). In addition, there are two special concepts associated with the modern type curve theory (the rate integral function and the rate integral derivative function) which reduce the noisiness of the production data, but may attenuate the reservoir signal at the same time. The rate integral function is a cumulative average rate, while the rate integral-derivative function is defined as the semi-logarithmic derivative of the rate integral function with respect to material balance time (Fekete, 2013b).

Fig. 20 shows Blasingame's integral-derivative typecurves for fractured horizontal wells, and Fig. 21 illustrates the rate integral concept.

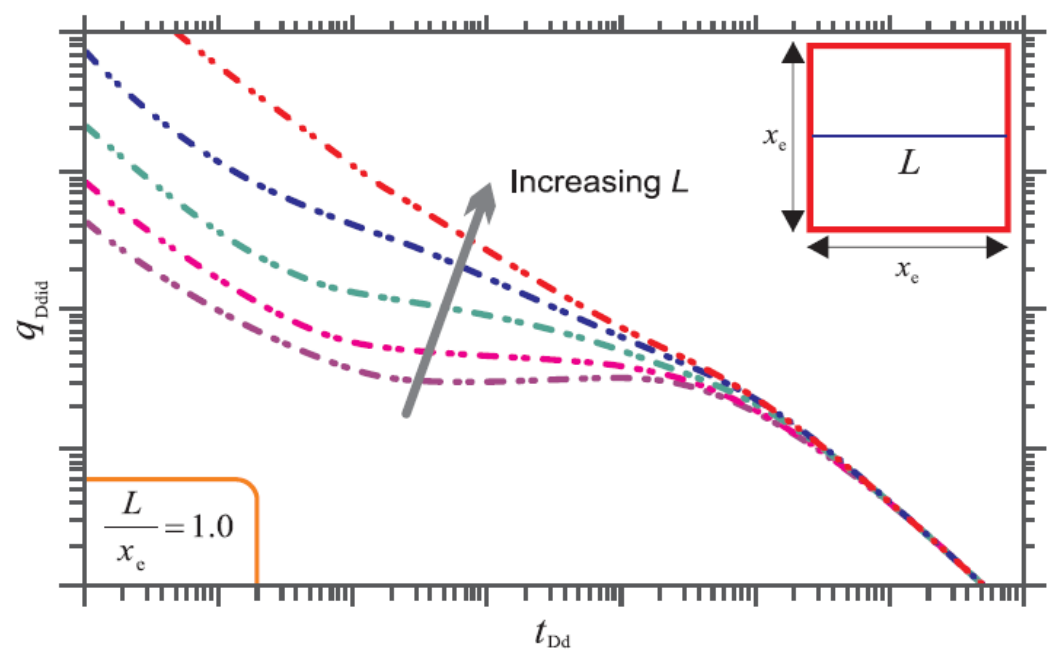

Fig. 20-Blasingame integral-derivative for fractured horizontal wells (Fekete, 2013a). 


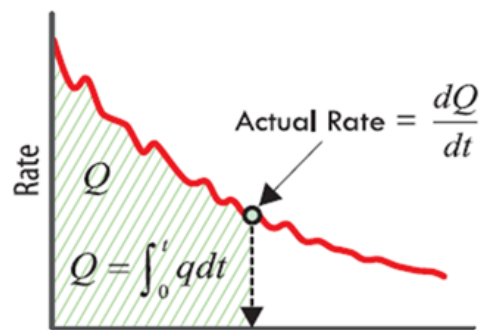

Actual Time $(t)$

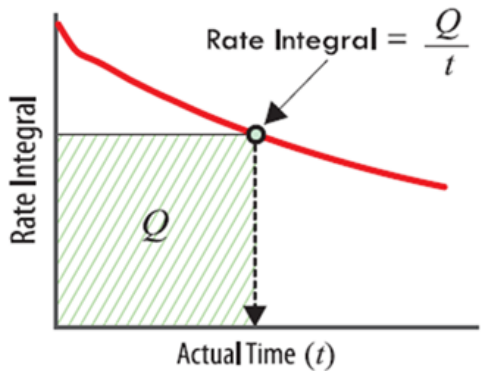

Actual Time $(t)$

Fig. 21-Illustration of the concept of rate integral (Fekete, 2013a).

\subsection{Flowing Material Balance}

The Flowing Material Balance method (FMB), proposed by Mattar and McNeil (1995), is a simple linear plot of Normalize Rate versus Normalized cumulative production used to estimate volumes in-place in oil and gas wells by extrapolating the straight line formed during boundary-dominated flow (Anderson et al., 2010). Opposite to the traditional material balance method, which requires static reservoir pressures measurements, the FMB method is based on flowing pressures instead of static pressure. This special feature avoids production losses during measurements (Mattar and Anderson, 2005). As pointed out by Mattar and Anderson (2003), and Thompson et al. (2011), ultimate recoveries may only be predicted with confidence after boundaries (BDF) conditions have been reached-when all the pore volume have been connected, and the reservoir is flowing under volumetric depletion-so the pressure at every point in the reservoir declines at the same rate. Nevertheless, if BDF is still not observed in the diagnostic plots, FMB may be used to estimate the minimum (conservative) volume of fluids inplace, assuming that depletion will be presented right immediately after the transient flow. The FMB technique was initially designed for constant rate wells flowing at boundary conditions (stabilized or pseudo-steady-state). However, Mattar and Anderson (2005) introduced an extension of the FMB, known as Dynamic Material Balance (DMB), which extends the application of this technique to wells with variable production rates. In practice, the DMB technique converts flowing pressures to average reservoir pressures at each time step. It 
means that $\mathrm{DMB}$ is an indirect method of estimating average reservoir pressures without shutting-in wells. As a result, a continuous plot of average static reservoir pressures in function of time may be obtained. Finally, hydrocarbon volumes can be estimated by applying the traditional material balance technique. Fig. 22 shows an example of the application of the DMB method in a gas well.

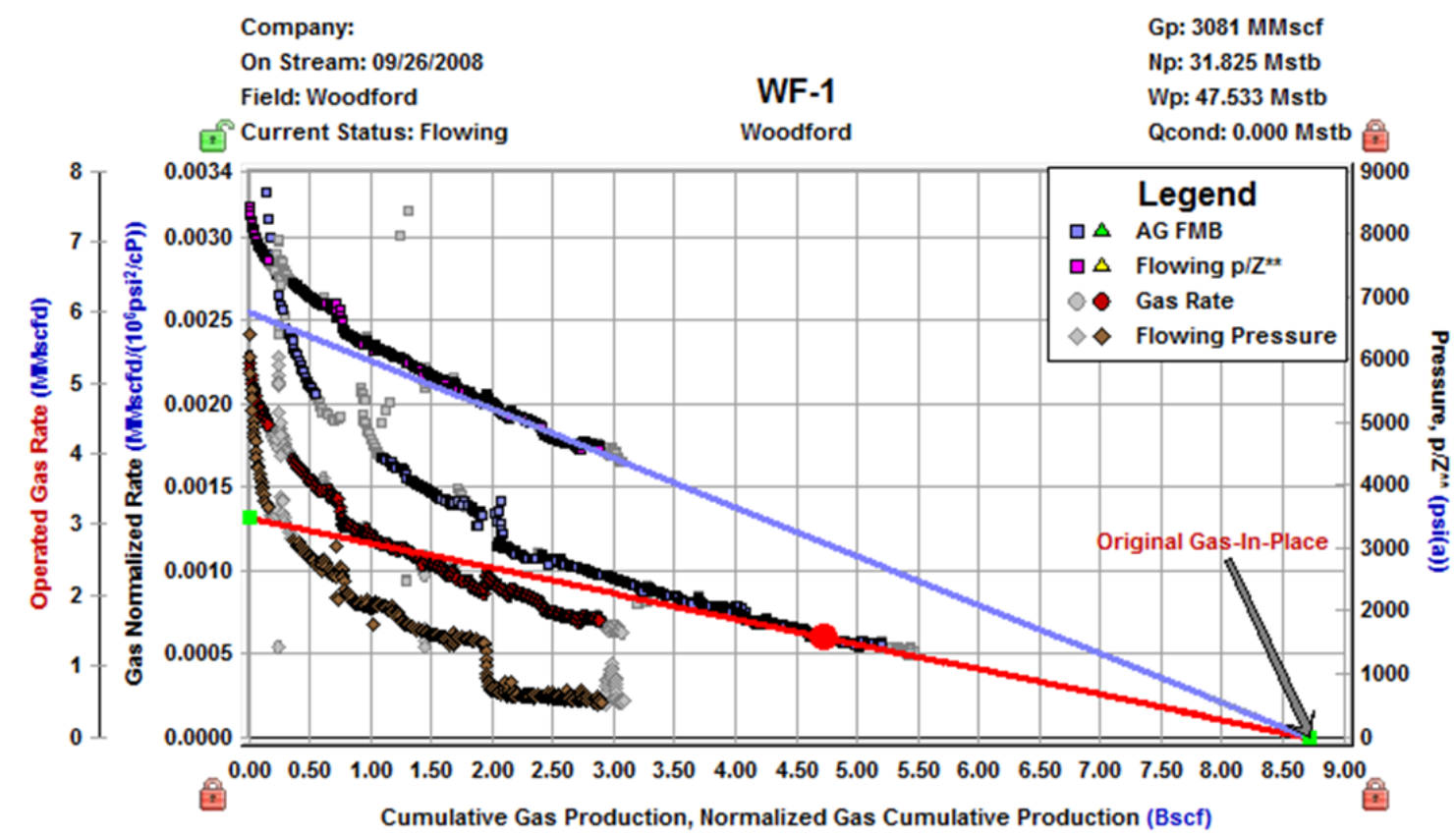

Fig. 22-Application of the dynamic material balance technique to a shale gas well.

\subsection{Analytical Models (Multi-Fractured Horizontal Wells)}

Horizontal wells with multiple hydraulic fractures (MFHW) are the most common completion technique in most of the unconventional plays, because they provide an effective solution to the low production performance of these complex reservoirs. In fact, in most cases this is the unique solution to economically produce them (Clarkson, 2013b). Analyzing the 
production behavior of these wells represents a difficult challenge because of the intricate flow patterns of the MFHW along with the other multiple complexities associated to the process (i.e., dual porosity effects, multi-layer behavior, reservoir heterogeneities, desorption effects, multi-phase flow and non-static absolute permeability) (Clarkson and Pedersen, 2010). Different analytical models have been created to overcome with these problems, including special options to include most of these special features. 


\section{PRESSURE NORMALIZED DECLINE CURVE ANALYSIS}

As previously mentioned in Section 2, one of the main drawbacks of the DCA methods is that they are based only on production rates, relying on the assumption of stable flowing pressures. Since this stabilized state is not reached rapidly in most cases, the applicability of these methods and the reliability of their solutions may be compromised. Examples of such situations are unconventional wells with short or discontinuous production histories, variable operating conditions, and high pressure, rate-controlled wells. In these particular cases, important reservoir productivity signals are expressed through both pressure and rate decline trends. Therefore, ignoring flowing pressures and using only conventional rate-time techniques to interpret these cases may cause misinterpretations and erroneous reserve estimations (Anderson, D.M. et al., 2012; Mattar and Anderson, 2003). As pointed out by Mattar and Anderson (2003), there is a possibility of over-predicting EUR if a steeply declining pressure is observed, even if a conservative exponential decline is applied. In addition, normalizing production rates with flowing pressure drops $(q / \Delta p)$ incorporates the effects of back pressure changes into the reservoir analysis.

More complex and advanced techniques are available to overcome the restrictions in DCA mentioned previously. The main difference is that these techniques use analytical and numerical models to analyze production rates and flowing pressures. Although those methods are more rigorous and provide some advantages over DCA techniques, a deep understanding of reservoir characteristics is required (some of them are known only approximately, and others are completely unknown). Considering that there is usually high uncertainty associated with most of the reservoir properties needed to use these complex methods, high uncertainty should correspondingly be expected in forecasts using this approach. Moreover, as the advanced analytical and numerical models depend on copious inputs, there is a high probability that different combinations of those parameters could generate equivalent and acceptable history matches, but different production forecasts and EUR estimations. Therefore, nonunique solutions may be expected (Anderson, D.M. et al., 2012). 
The new pressure-normalized DCA techniques (PN-DCA) may provide a suitable solution to the previously stated problem, without recurring to complex analytical and numerical models. These techniques preserve the simplicity and the other advantages of the traditional DCA methods because their fundamentals are fairly similar, with the only difference being that pressure data is included in the analysis. Additionally, as pointed out by Thompson et al. (2011), decline curve analysis is the favorite language between reserve evaluators, even though advanced analytical models provide a better insight of the reservoir performance and a more flexible forecasting capacity. In general, as the pressure-normalized decline curve analysis (PN-DCA) relies on rate and pressure data, an improved forecasting capacity may be expected. Additionally, unlike decline curve analysis, different future operating conditions can be assumed and multiple ultimate recoveries may be predicted.

\subsection{Pressure Normalization of Production Rates}

The pressure-normalized decline curve analysis is grounded on the concept that pressure-normalized rates (PNR), or their reciprocal (RNP), may better represents the real production performance of wells flowing under unstable conditions. This theory is supported by the fluid flow analytical solution, which relates production rates, flowing pressures, and the reservoir productivity properties, as a function of time. Considering that linear flow is the dominant flow regime in most of unconventional reservoirs, the analytical solution for transient linear flow is presented as main reference (See Eqs. 42 through 44). Note that for gas wells, pseudovariables (pseudopressures and pseudotime) should be included in the analysis to account for the changing gas properties (Agarwal et al., 1999; Clarkson, 2013a; Nobakht and Clarkson, 2012b). Initially, Eq. 42 was derived assuming constant flowing pressures. Thus, the independent variable was the reciprocal of production rate, 1/q (El-Banbi and Wattenbarger, 1998; Wattenbarger et al., 1998). This is a fair assumption for ultra-low permeability wells, which produce under very high drawdowns to maximize their productivity (Fekete, 2013b; Nobakht et al., 2010). However, the reality is that this condition is not reached easily in most cases. Therefore, normalized variables (PNR or RNP) have been introduced to deal with production analysis where flowing pressures and rates change through time (Anderson et al., 
2010; Clarkson, 2013a; Fekete, 2013b; Nobakht and Clarkson, 2012a). In fact, most of the modern production analysis techniques (e.g., Agarwal-Gardner and Blasingame typecurves, and flowing material balance) rely on pressure-normalized rates, instead of production rates only.

In general, as demonstrated by the transient linear flow solution, pressure-normalized rates are closely related to the reservoir properties affecting the production performance process, and have the special advantage to account for unstable flowing conditions. Therefore, pressure-normalized rates are expected to better represent the real well deliverability potential, in comparison with production rates only. As a result, more reliable production performance predictions may be obtained if both signals, production rates and flowing pressures, are included in the analysis.

$$
\begin{aligned}
& \frac{P_{i}-P_{w f}}{q}=m \sqrt{t}+b^{\prime} \ldots . . \\
& m=\frac{315.4 T}{\sqrt{\left(\phi \mu_{g} c_{t}\right)_{i}}} * \frac{1}{h x_{f} \sqrt{k}} . . . \\
& m=\frac{31.3 B}{h x_{f} \sqrt{k}} * \sqrt{\frac{\mu_{o}}{\phi c_{t}}} \ldots \ldots . . .
\end{aligned}
$$

The three normalization functions (PN, $\triangle \Psi N$, SPN) used in this work to generate the pressure-normalized rates are presented below (Eq. 45 through 47). Eq. 46 applies only for gas wells. Note that Eq. 47 is an additional function introduced in this study. This relationship was deducted based on the approximation of the productivity index for gas wells in the low pressure region (i.e., both flowing pressure and average static reservoir pressure are lower than 2000 psi), where the gas pressure-dependent functions exhibit a linear relation with pressure. However this function will be tested on both oil and gas wells.

$$
P N=\frac{q}{\Delta p}=\frac{q}{p_{i}-p_{w f}}
$$




$$
\begin{aligned}
& \Delta \psi N=\frac{q_{g}}{\Delta \psi}=\frac{q_{g}}{\overline{\psi_{l}}-\psi_{w f}} . \\
& S P N=\frac{q}{\Delta p^{2}}=\frac{q}{p_{i}{ }^{2}-p_{w f}{ }^{2}}
\end{aligned}
$$

\subsection{Pressure Corrected Rates}

Duong (2010) proposed a new decline analysis model suited for transient flow regimes. As part of his technique's workflow, pressure corrected rates are used in diagnostic plots to compensate for the early choke-back effect and to improve the identification of the linear flow regime (Fig. 23), but not to improve the forecasting capacity of the DCA model. Thus, Duong's model relies only on production rates. In this work, the same technique will be applied, along with the previously mentioned pressure normalization functions, to trying to improve the forecasting capacity of the DCA models (see Eq. 48).

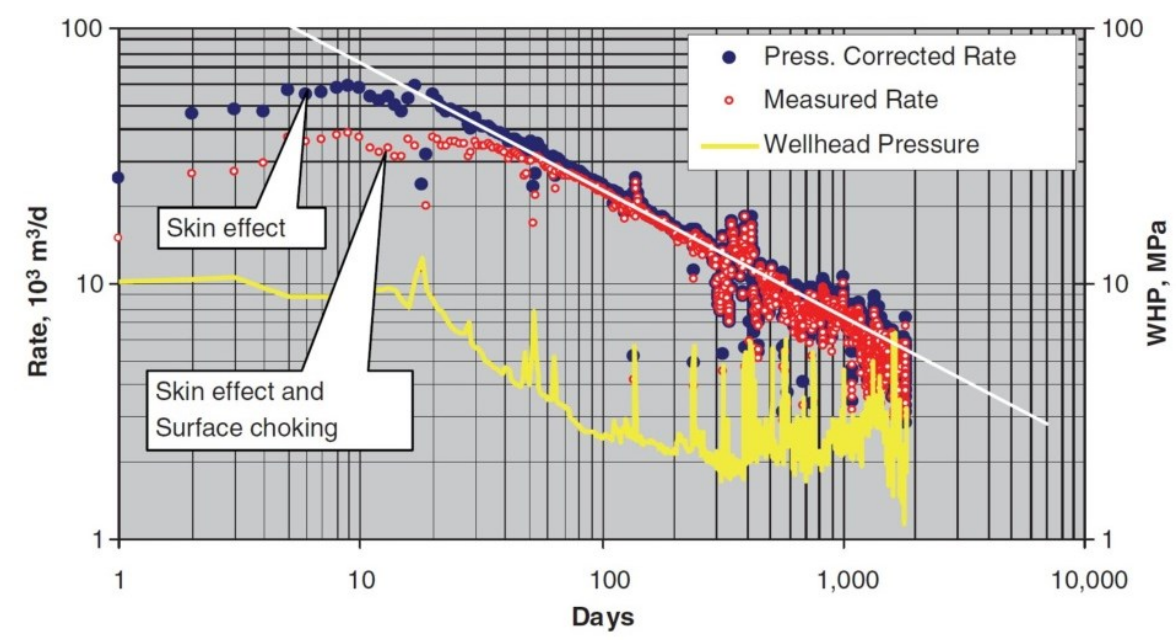

Fig. 23-Application of pressure corrected rates in diagnostic plots to compensate for the early choke-back effect

(Duong, 2010). 


$$
q_{c o r r}=q_{o b s} *\left[\frac{P_{i}-P w f_{s t b}}{P_{i}-P w f_{o b s}}\right]
$$

\subsection{Applicability of Pressure-Normalized Decline Curve Analysis}

In general, PN-DCA techniques can be applied to the same cases where DCA methods are applied, but their main strength is the capability of providing solutions to those special cases where regular DCA models are constrained. A better description of such cases is presented below.

\subsubsection{Variable Operating Conditions}

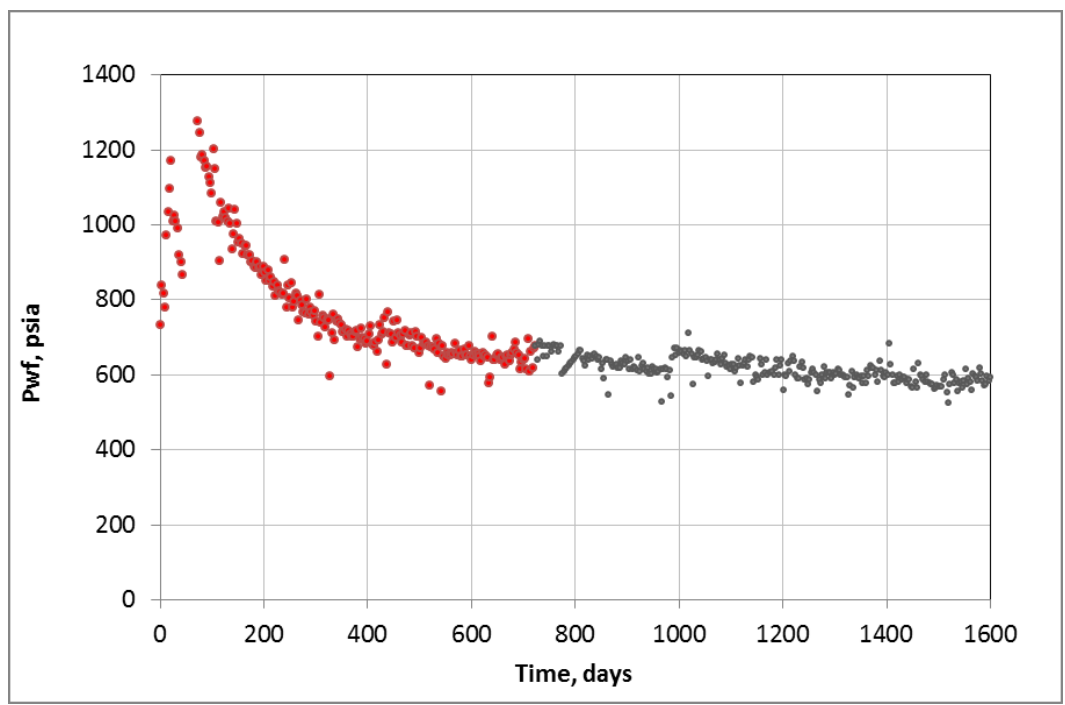

Fig. 24-Plot of Pwf vs. time for a high pressure rate restricted shale well.

During their productive life, most wells are subject to stimulation operations to improve their productivity or are submitted to variations in the operating conditions resulting from different factors, such as workover activities, change of production strategy and 
wellbore/production facilities reconfiguration. Consequently, the constant flowing condition assumption on which DCA methods rely is not valid anymore. For this reason, these rate-time relations should not be applied to unstable historic data to forecast future productions, because unrealistic estimations could be yielded. As an example, Fig. 24 shows flowing pressure of a shale well. Note that bottomhole flowing pressures declined during the firsts two years of production (red points). Thus, in theory, decline curve analysis techniques should not be applied until a stable flowing pressure condition is reached. One of the most important advantages of PN-DCA is its ability to disassociate future performance predictions from actual operating conditions. As flowing pressures are included in the analysis, the predicted decline curve $(q / \Delta P)$ may be used to estimate production performance at different flowing pressures.

The impact of these unstable conditions on the production performance will depend on their severity and duration. Sometimes, operational fluctuations involve either short shut-ins or slight variations of the flowing conditions, but in some other cases, flowing conditions are dramatically altered or significant downtimes are produced by long closures. When major changes occur, flow regimes could equally change. Agnia et al. (2012) demonstrated that when the production rate is severely reduced, a new transient effect is generated in the reservoir once again, even if the well has been flowing in BDF. One common situation is the case of a well that is reopened to production after a long shut-in. Accordingly, a new transient effect may be restarted in the wellbore, even if the well had been producing in BDF regime for long time previous to the closure (Fekete, 2013b). Therefore, it is highly recommended to perform a flow regime diagnosis to identify alterations in the fluid flow patterns after significant downtimes. If unsteady-state (transient) flow is observed, a time reinitialization is advised. This method was proposed by Fetkovich to evaluate production data with multiple transient periods. Basically, it consists of resetting the start time of each transient period to zero, as well as its production history. On the other hand, when a well is submitted to any stimulation job, it is highly probable that its deliverability potential is affected and consequently, the remaining recoverable reserves are equally altered. So, the production analysis must be reinitialized and any production data (rates and pressures) collected before this moment is no longer representative of the new reservoir performance and cannot be used to forecast future production trends. 


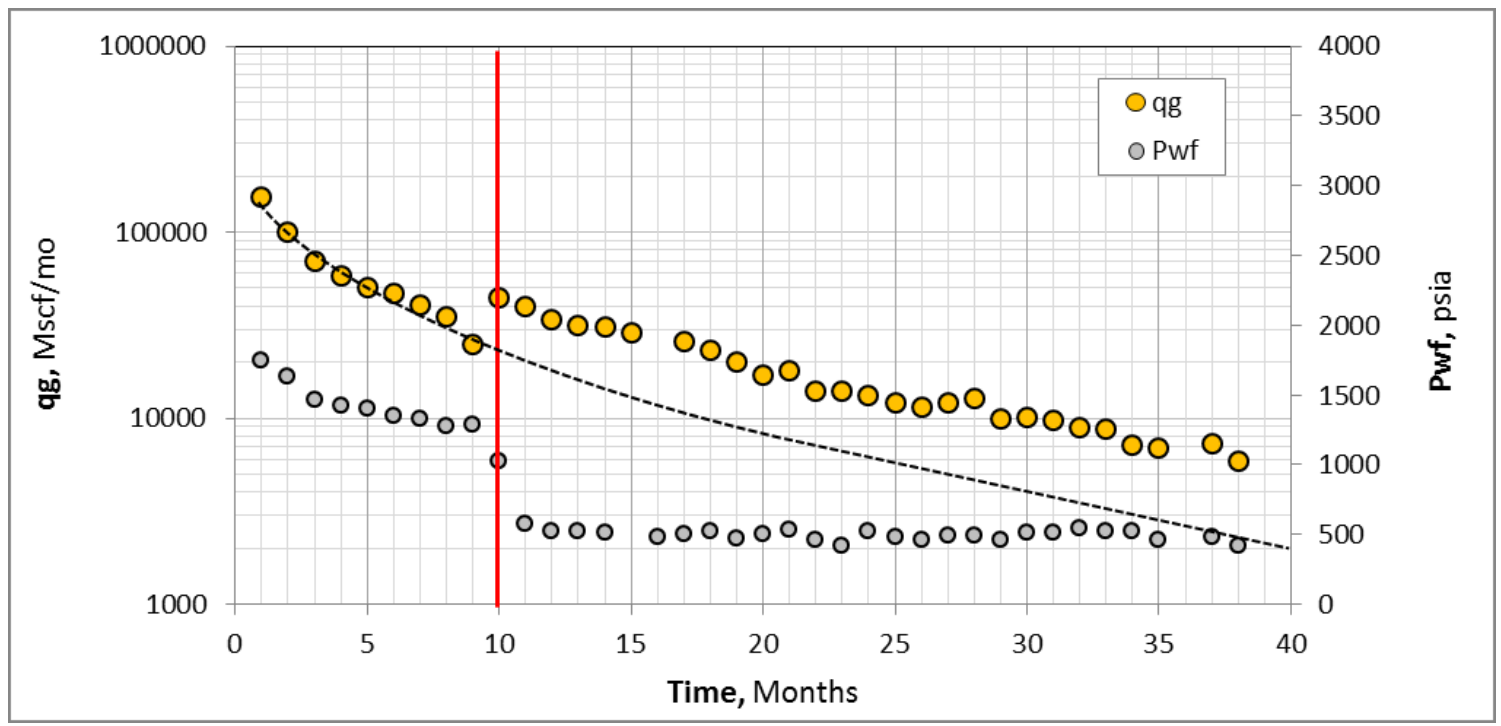

Fig. 25-Example of the effect of changing operating conditions on production rates.

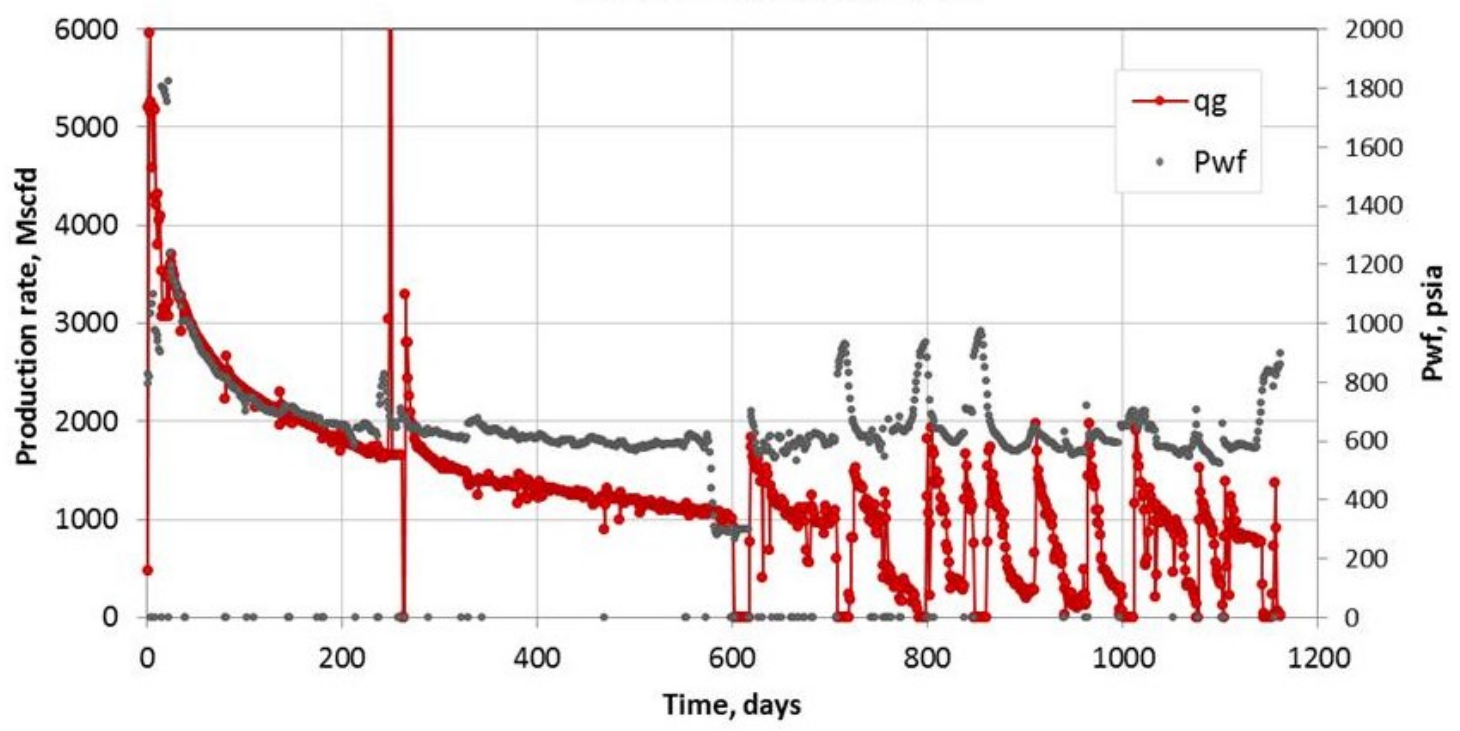

Fig. 26-Example of an unconventional shale gas well producing under variable operating conditions.

Additionally, there are other operations or phenomena that can alter the production performance of a well, invalidating any previous production analysis and forcing the restart of 
its production history, as well as any forecast yielded by it. Examples of such situations are: infill drilling (affecting drainage area), water breakthrough (affecting relative permeability) and completion reconfiguration (affecting effective flow area), among others.

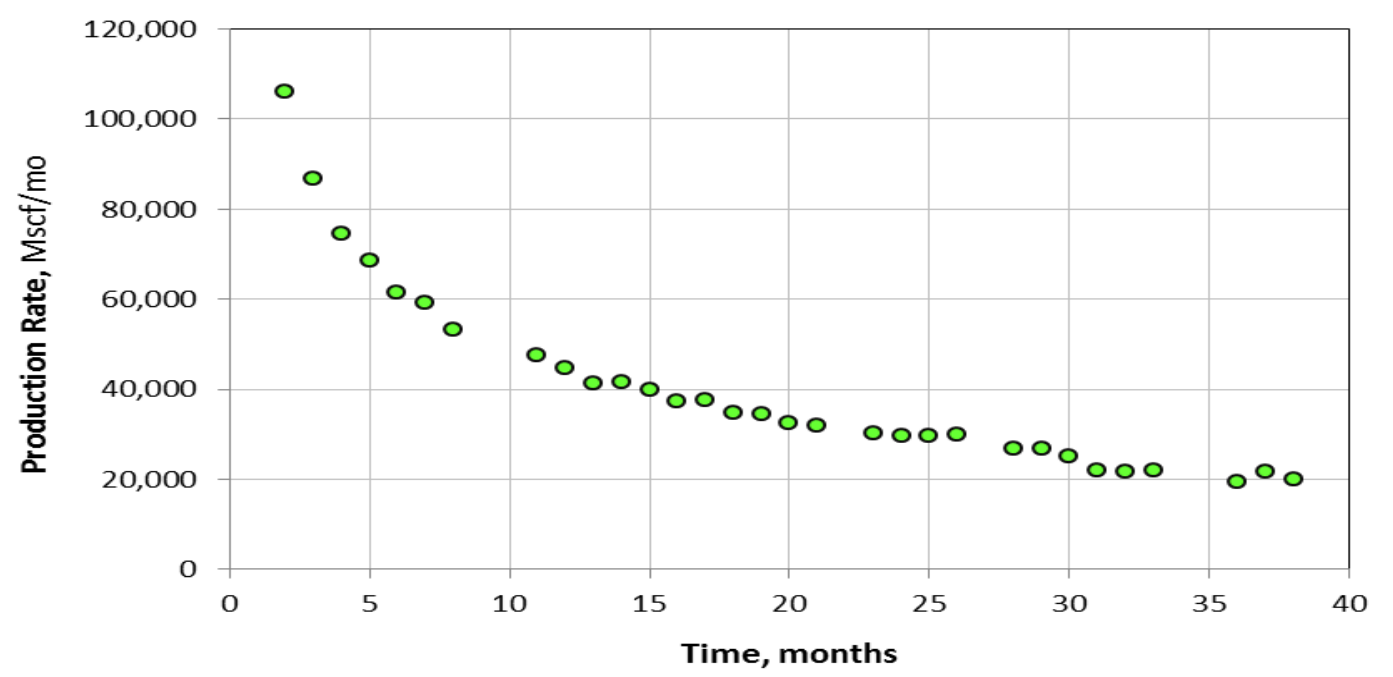

Fig. 27-Example of monthly averaged and filtered production rates.

As an example, Fig. 25 shows the effect of changing operating conditions. Production rates increase as an effect of decreasing flowing pressures. Using the first ten months of production to forecast future performance will result in underestimated predictions, as shown by the black dotted line. Fig. 26 shows daily flowing pressures and production rates from an unconventional shale gas well producing under very unstable operating conditions (i.e., a short shut-in after 260 days of production, and multiple closures and production changes after 600 days). When drastic operating fluctuations are presented during a significant time, production data may be severely affected and should not be used for production analysis purposes because its decline trend doesn't represent the real well performance with confidence. Fig. 27 shows average monthly production rates for the same well after smoothing filters have been applied, a common practice in the industry. Amazingly, all the inconsistences and production 
disturbances have apparently disappeared, and the data seems to be ready to be analyzed. Several questions may come up in this case. Has this data correction process managed to overcome all the production instabilities? Is this new production profile a true representation of the real reservoir performance? Could the future production behavior be reliably forecasted based on this data? Is the constant flowing pressure assumption of the DCA methods still valid? As each well is unique, most of the answers to these questions depend on each case and will have to be addressed by each interpreter. The PN-DCA techniques and the workflow presented in this work attempt to provide a better understanding of such phenomena, as well as an improved approach to get a more accurate solution to those complex cases.

\subsubsection{High Pressure Rate Restricted Wells}

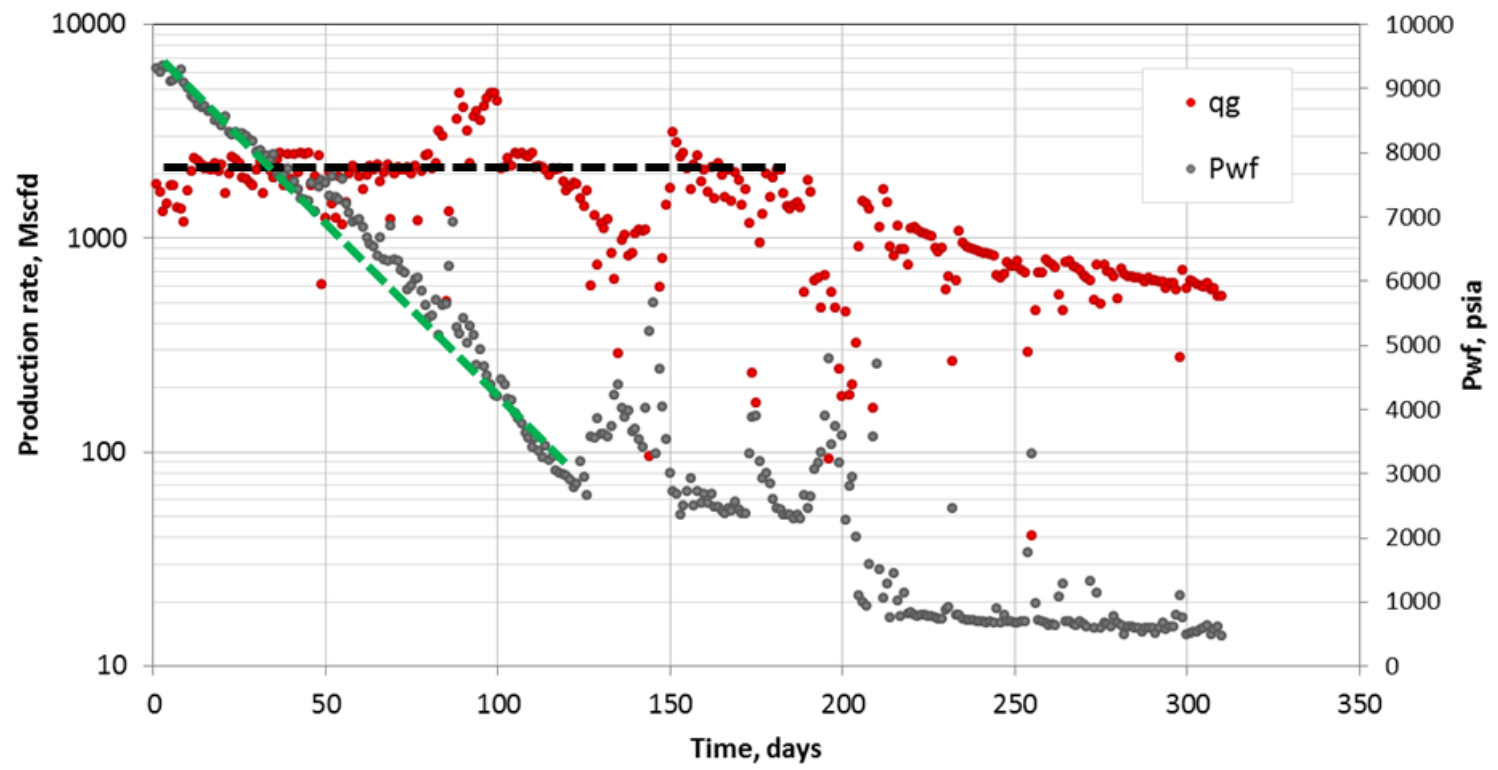

Fig. 28-Example of a high pressure, rate restricted gas well (EF-4).

As mentioned by Anderson, S. et al. (2012), production rates from some high deliverability or high pressure unconventional wells, such as those in Haynesville and Eagle Ford 
plays, are restricted during extended periods of time. In these cases, regular DCA methods are disqualified as production analysis tools because production rates are controlled to be kept constant during some time. Additionally, flowing pressures of those wells operating under restricted flow conditions exhibit a declining trend, violating the constant flowing pressure assumption of the DCA methods. For these reasons, rate-time techniques can only be applied in later production times when the required stable conditions are reached. Pressure normalized rates may be used instead of simple production rates to reproduce a decline curve, which can be used for DCA purposes. Fig. 28 presents an example of a high pressure, rate controlled gas well. Note that production rates and flowing pressures don't exhibit a natural decline trend. Instead, the average production rates are controlled to be nearly constant during months of production (see black dotted line), while flowing pressures decrease slowly (green dotted line).

\subsubsection{Short Production History}

Typically, wells flow at high rates-over its expected normal performance-during the beginning of its productive life, and in response, high decline rates are presented. Subsequently, those high production and declination rates continuously decrease until reaching more stable values. Simultaneously, bottomhole flowing pressures exhibits a similar behavior, decreasing steeply from the initial average pressure until reaching a more stable profile. In the case of multi-fractured horizontal wells in unconventional reservoirs, this behavior is attributable to the dominant transient linear flow. Early production is mainly driven by near wellbore productivity features (fracture network properties). This is considered an unstable period because the unusual high production and decline rates are far from representing the true long term reservoir potential. In addition, the constant flowing pressure assumption of DCA technique is not valid. Considering these reasons, production forecasts based on short production histories should be avoided. Ideally, reliable production analyses should be based on data from BDF, which is not the dominant flow regime in unconventional reservoirs (especially at early times). Unfortunately, unconventional wells with short and unstable production history frequently need to be analyzed, and interpreters are forced to find a solution. In most cases, DCA models are mechanically applied, and the resulting matched model 
is extrapolated to estimate ultimate recovery, regardless of the stability of flowing conditions. Of course, this common practice may yield unrealistic outcomes.

Fig. 29 shows an example of a shale gas well producing under changing flowing conditions (Variations in operating conditions are indicated by the dotted blue lines). Note that during the first 100 days of production, high production and decline rates are easily observed, while flowing pressures decrease continuously until reaching some stable conditions (after approximately 550 days of production). At the end, flowing pressures change once again in consequence of new induced operating conditions.

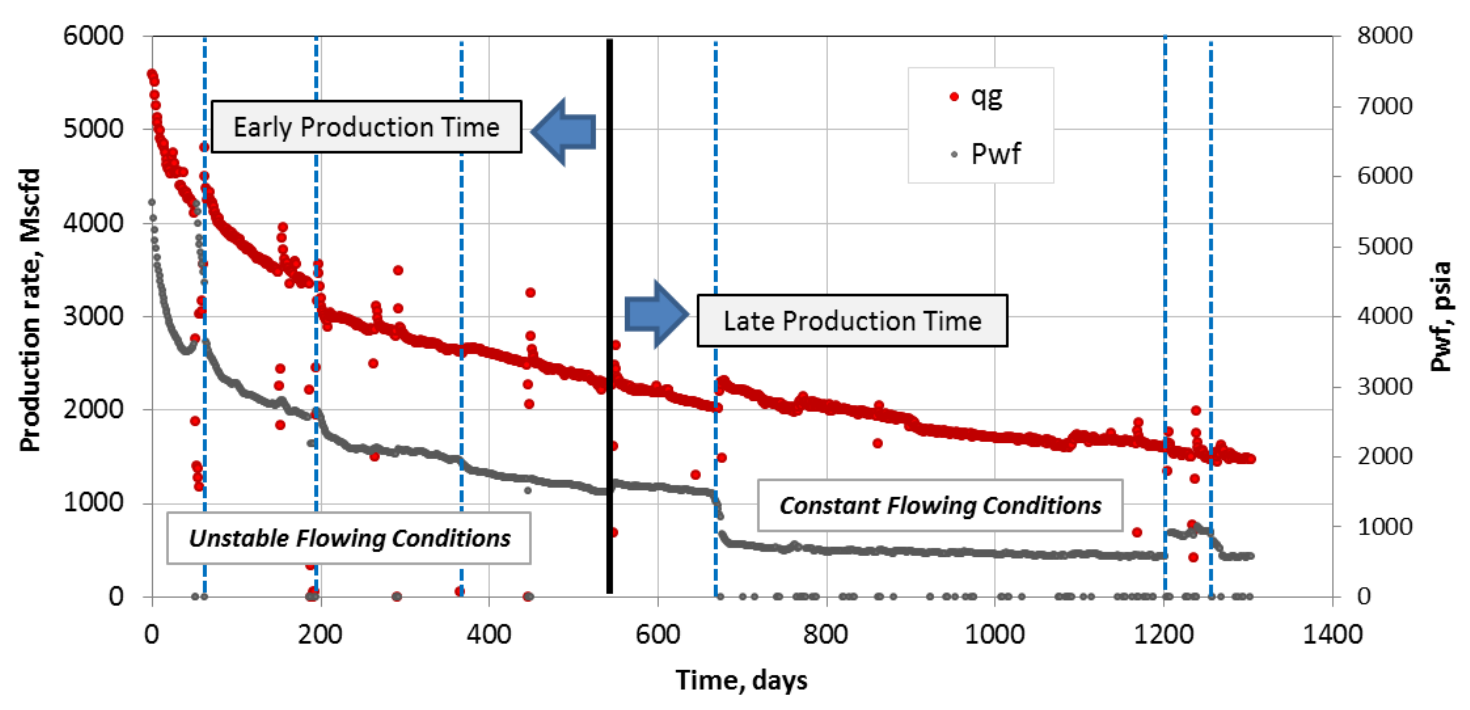

Fig. 29-Example of unstable flowing conditions at early production times.

The PN-DCA technique attempts to provide a more realistic approach to the big challenge of forecasting production and estimating ultimate recoveries to those wells with short and unstable production history. Even if the constant flowing pressure condition has been reached, unrealistic results may still be produced depending on how long the stabilized period is in comparison with the unstable early production period. Since it is extremely difficult to 
estimate how affected the forecast could be by the early time effect, it is highly recommended to try to correct this pressure effect. So, in any case better results could be obtained if production rates are corrected by pressure.

\subsubsection{Flow Regime Identification}

An important application of pressure-normalized rates is the flow regime identification. The arbitrary application of DCA models without a previous identification of flow regimes may produce unrealistic production forecasts, because each model has specific flow regime limitations that need to be considered seriously. Usually, flow regimes are characterized using basic diagnostic plots that include only production rates. The most common flow regimes diagnostic plot is Log q vs. Log t. Nevertheless, because important flow pattern signals are expressed through both flowing pressures and production rates, this plot may be a misleading approach in some cases. Hence, it is highly recommended to use diagnosis plots based on pressure-normalized rates, instead of production rates, to reliably identify flow regimes before proceeding with decline curve analysis.

\subsection{Production Forecasting Techniques For Pressure-Normalized Rates}

\subsubsection{Decline Curve Analysis Based on Normalized Rates}

This technique, called pressure-normalized decline curve analysis (PN-DCA), consists of performing decline curve analysis (DCA) using pressure-normalized rates (PNR) instead of real production rates. Note that in this study PNR refers to any of the pressure normalization methods (PN, $\Psi N R$, and SNR). Different pressure normalization methods can be used to transform regular production rates into pressure-normalized rates. In this study, three pressure normalization functions $\left(\Delta \mathrm{p}, \Delta \psi\right.$, and $\left.\Delta \mathrm{p}^{2}\right)$ are proposed along with an additional pressure correction method $\left(q_{\text {corr }}\right)$. Although the square pressure normalization method was specially deducted for gas wells (See Section 4.1), it will be tested in oil wells likewise. A summary of these methods are presented in Table 7. Unlike pressure normalization functions, the pressure 
correction method generates production rates, which have been corrected by applying a pressure correction factor $(\triangle \mathrm{PF})$ that accounts for flowing instabilities (i.e., flowing pressures different to the stable flowing pressure, if observed).

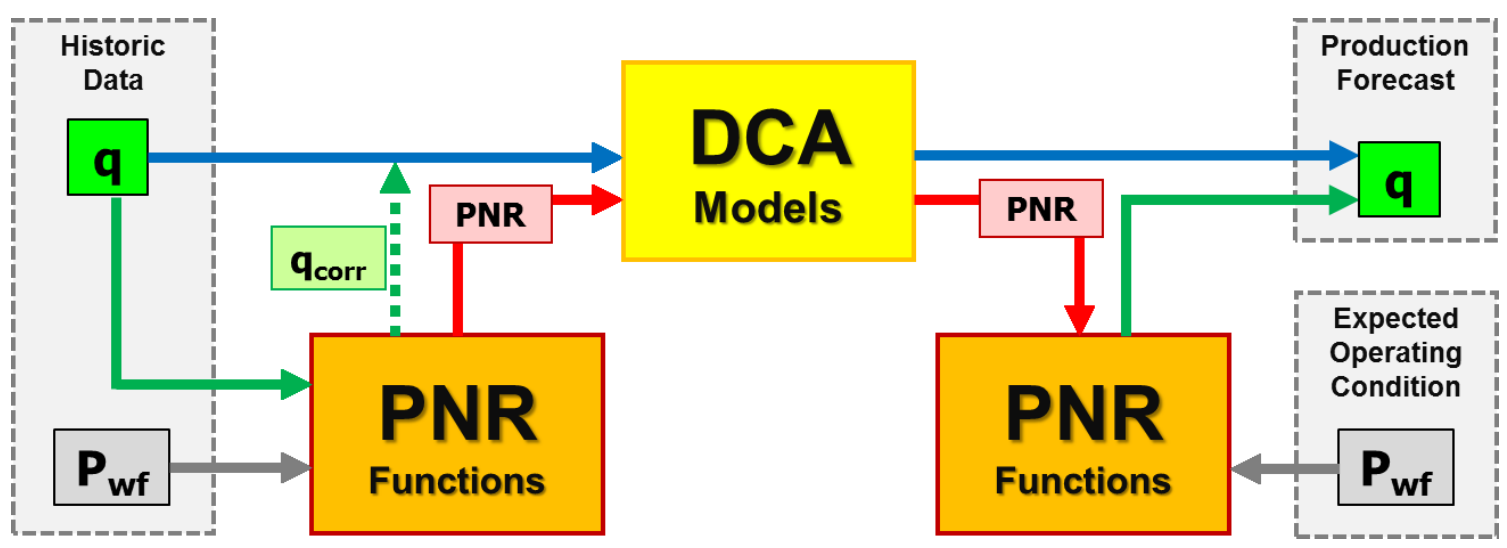

Fig. 30-Illustration of the application of decline curve analysis using pressure-normalized rates. Red and green lines represent PN-DCA method, while blue lines correspond to the traditional application of DCA models.

The application of decline curve analysis using PNR is illustrated in Fig. $\mathbf{3 0}$ and is described as follows:

- PNR are generated by the respective pressure normalization functions $(\Delta p, \Delta \psi$, $\left.\Delta p^{2}\right)$, and corrected rates $\left(q_{\text {corr }}\right)$ are generated by the pressure correction method.

- Forecasting is performed by applying the traditional DCA models to the generated PNR and $\mathrm{q}_{\mathrm{corr}}$ profiles.

- PNR forecasting outputs are pressure-normalized rates, just like input data. Therefore, these values must be converted back to real production rates. Then, the forecasted PNR must be multiplied by the corresponding delta-pressure function (i.e., $\Delta p, \Delta \psi, \Delta p^{2}$ ), which represents the expected future operating (flowing pressure) conditions. Contrary to traditional DCA models, which 
assume constant flowing conditions, PN-DCA forecasts are not restricted to historic operating condition. Therefore, multiple production trends can be forecasted, assuming diverse operating scenarios.

- In the case of corrected rates $\left(\mathrm{q}_{\text {corr }}\right)$, the previous step is not necessary because forecasting outputs are real production rates. Therefore, predictions based on this technique don't need any transformation after forecasting analysis. However, this method lacks the advantage of the PN methods to disassociate future production estimations from past operating conditions.

Table 7-Summary of pressure normalization methods and pressure-normalized rates (PNR)

\begin{tabular}{|c|c|c|}
\hline Normalization Method & Oil Wells & Gas Wells \\
\hline $\begin{array}{l}\text { Pressure Normalization } \\
\qquad \text { (PN) }\end{array}$ & $P N=\frac{q_{o}}{\Delta p}=\frac{q_{o}}{p_{i}-p_{w f}}$ & $P N=\frac{q_{g}}{\Delta p}=\frac{q_{g}}{p_{i}-p_{w f}}$ \\
\hline $\begin{array}{l}\text { Pseudopressure Normalization } \\
\qquad(\Delta \psi N)\end{array}$ & -- & $\psi N R=\frac{q_{g}}{\Delta \psi}=\frac{q_{g}}{\overline{\psi_{l}}-\psi_{w f}}$ \\
\hline Square Pressure Normalization & $S N R=\frac{q_{o}}{\Delta p^{2}}=\frac{q_{o}}{p_{i}{ }^{2}-p_{w f}{ }^{2}}$ & $S N R=\frac{q_{g}}{\Delta p^{2}}=\frac{q_{g}}{p_{i}{ }^{2}-p_{w f}{ }^{2}}$ \\
\hline (SPN or PN2) & & \\
\hline $\begin{array}{l}\text { Pressure Correction Factor } \\
\qquad(\Delta \mathrm{PF})\end{array}$ & $q_{c o r r}=q_{o b s} *\left[\frac{P_{i}-P w f_{s t b}}{P_{i}-P w f_{o b s}}\right]$ & $q_{c o r r}=q_{o b s} *\left[\frac{P_{i}-P w f_{s t b}}{P_{i}-P w f_{o b s}}\right]$ \\
\hline
\end{tabular}

\subsubsection{Harmonic Decline of PNR vs. Cumulative Production}

Anderson, S. et al. (2012) proposed a new decline curve analysis method based on pressure-normalized rates to estimate ultimate recoveries and future production of high 
pressure, rate restricted wells. This method is grounded in the empirical observation that some wells exhibit a linear relationship between pressure-normalized rates and cumulative production when plotted on a semi-log scale; i.e., harmonic decline (See Fig. 31). Therefore, production is forecasted by extrapolating the harmonic decline of the pressure-normalized rates. Although this is the first published DCA method to include normalized rates in the analysis, its application may be restricted to some specific cases, like high pressure, rate restricted wells exhibiting harmonic decline. Additionally, this method assumes that harmonic decline will continue during the entire well life. In fact, even if this is a fair consideration, there is no physical concept supporting this assumption of harmonic decline.

In this work, the harmonic decline extrapolation method will be applied and compared with the other DCA and PN-DCA techniques. In the case of gas wells, this technique will be implemented using pressure-normalized rates (PNR), as well as pseudopressure-normalized rates ( $\Psi N R)$. Oil wells will be analyzed using PNR only.

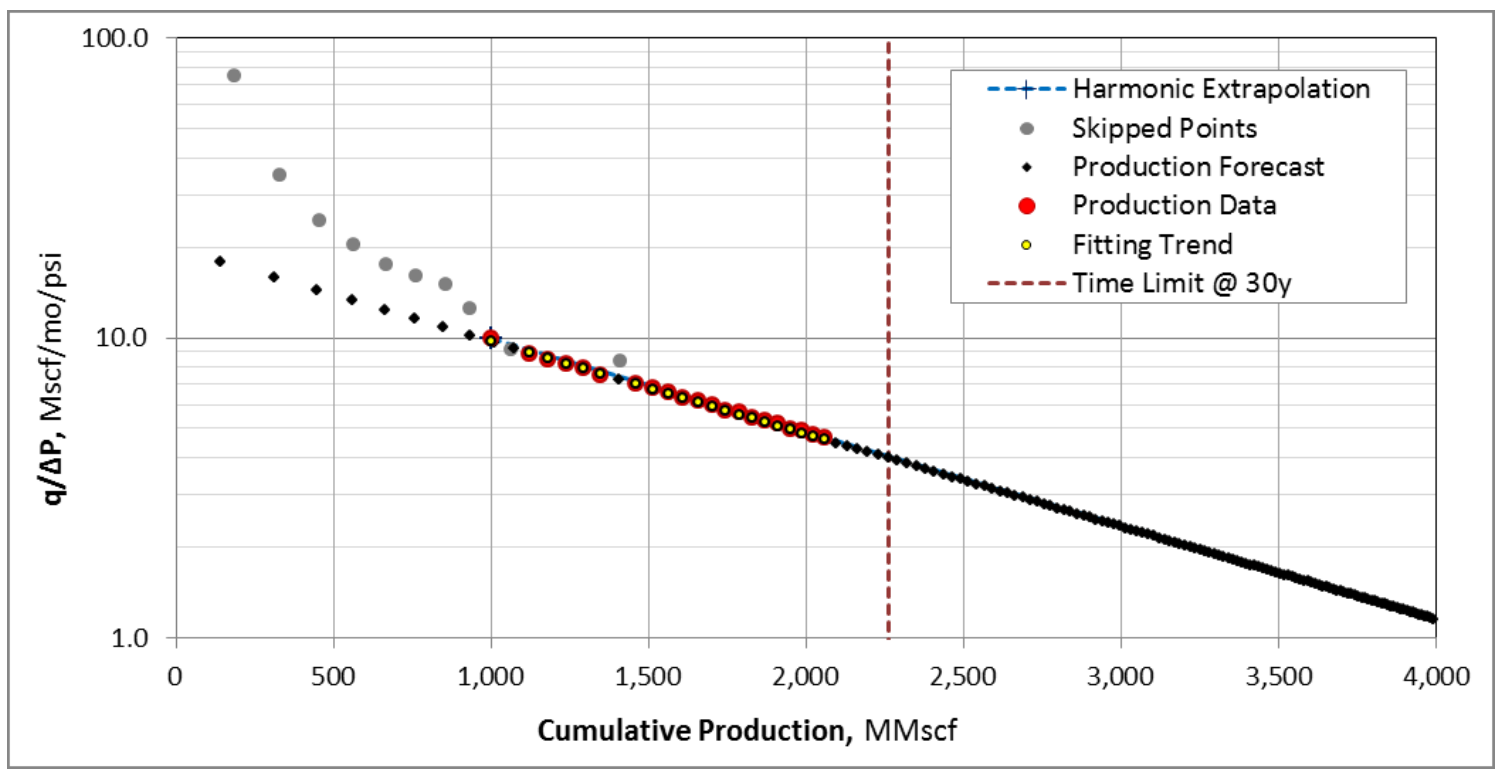

Fig. 31-Pressure normalized rate versus cumulative production. 


\section{FIELD DATA ANALYSIS AND DISCUSSION OF RESULTS}

Initially, this section presents a detailed description of the methodology used in this study to evaluate the applicability of pressure-normalized decline curve analysis (PN-DCA) as a production forecasting technique suited for the interpretation of unconventional wells. At the end, the results of this evaluation are presented, including flow regimes diagnosis, an assessment of the pressure-normalized harmonic decline method, and the comparative evaluation of the different DCA models and techniques based on pressure-normalized rates.

\subsection{Description of the Analysis Workflow (Methodology)}

\subsubsection{Production Data Diagnosis and Preparation}

This is the first step of the analysis workflow (See Fig. 32). The target of the data diagnostic process is to assure that production analyses are developed using high quality data. Thus, representative results may be equally expected. In practice, the data diagnostic process consists of identifying anomalous events during wells life that affect flowing conditions and that could represent a violation of the fluid flow theory assumptions on which production analysis techniques are based. Since the application of DCA techniques is restricted to specific cases, where certain conditions must prevail, violating such assumptions may yield unreliable and meaningless results deviating from the true reservoir performance (See Section 2).

Unlike pressure transient analysis (PTA), where production/pressure data is accurately obtained as part of a controlled operation, production analysis (PA) is performed using surveillance and monitoring data acquired in variable operating conditions, and in most cases it is measured with questionable accuracy. Therefore, when analyzing production data, interpreters must be aware of possible biases or misinterpretations due to poor quality input data (low frequency/low resolution production rates). However, while production rates are 
continuously recorded most of times-independent of its quality, pressure historic data for production analysis purposes is not frequently available or it is totally inexistent in some cases (Anderson et al., 2006).

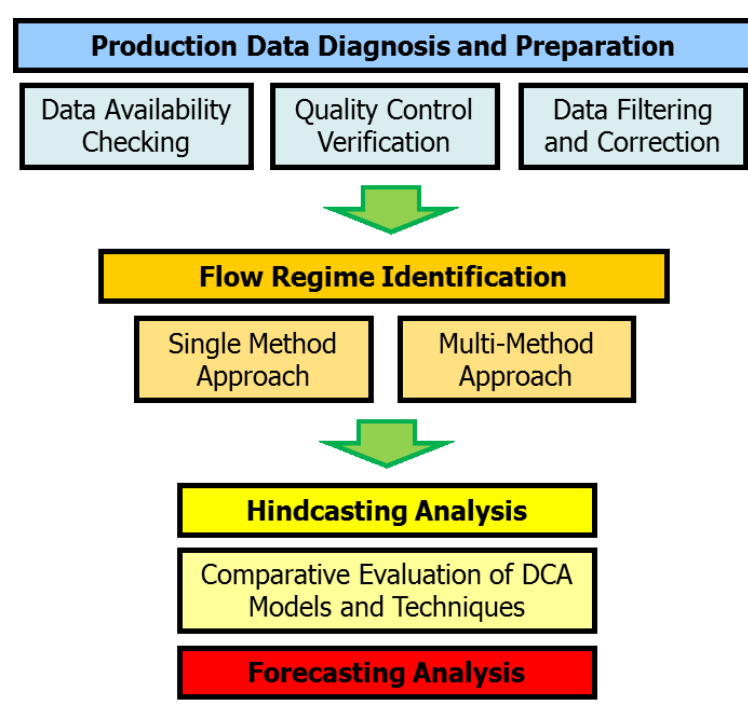

Fig. 32-Workflow of the PN-DCA Methodology

\subsubsection{Data Availability Checking}

The purpose of this first step is verifying if the data required for the analysis is available. Although DCA methods rely only on production rates, PN-DCA technique needs production rates, flowing pressure data, and in some cases, fluid and reservoir properties are also required. Usually, surface flowing pressures are not an exact indication of sandface conditions because of the multiple phenomena happening at wellbore during fluids production (particularly in the case of multiphase flow). Therefore, PN-DCA techniques should be applied using bottomhole flowing pressures instead of tubing head pressures (THP). If downhole pressures have been measured, which is an ideal situation, next information is needed:

- Production rates. 
- Bottomhole flowing pressure

- Average initial reservoir pressure

If downhole flowing pressure (Pwf) measurements are not available, wellhead flowing pressure data (i.e., casing or tubing head pressure), as well as some basic fluid and reservoir properties are required to calculate pressure losses through wellbore/completion system, and to estimate sandface flowing pressures. Pressure differences between surface and bottomhole reference points are generated by three main effects: hydrostatic pressure loss, frictional pressure loss and kinetic pressure loss (this last one is usually ignored). In normal production operations, upward fluid flow must overcome two opposite pressures in its pathway from reservoir to surface: the backpressure exerted by the effective hydrostatic column and friction losses due to physical interaction between the fluids and the wellbore surfaces (Fekete, 2013b). Most common pressure loss correlations are Fanning for single-phase flow, and Beggs \& Brill, Gray, Hagedorn \& Brown, and Petalas and Aziz for multiphase flow.

The following list describes the input data required to analyze each well if bottomhole flowing pressures are unknown. Note that most of this information is required by pressure loss correlations.

- Production rates.

- Wellhead flowing pressure. Casing or tubing head pressures

- Average initial reservoir pressure

- Formation and fluid properties. Basic PVT Information is needed. Otherwise, basic fluid properties are required to generate synthetic PVT from reservoir fluid's correlations. Input data varies depending on each correlation. Usually, surface and bottomhole temperatures, as well as average fluid and reservoir properties (i.e., porosity, net pay, gas gravity, API, GOR, bubble pressure and fluid saturations) are needed. 
- Wellbore diagram. Deviation survey is needed, if possible. Otherwise, basic measurements (MD and TVD) of vertical and horizontal well section, tubing and casing dimensions, and producing (open to flow) section length are required.

According to Mattar and Anderson (2003), the accuracy of the flowing pressures measurements is less important (has a lower effect on the analysis) when the drawdown is higher than $50-60 \%$ of the static reservoir pressure. Additionally, he stated that casing pressures are more suitable to calculate bottomhole flowing pressures than tubing pressures. Preferably, data sources should be known, in order to establish the reliability and limitations of the gathered information. In the case of production rates, accurate individual well metering is strongly required, if possible. Otherwise, the reservoir signal and the representativeness of the analysis results could be meaningfully compromised.

Another important issue is the data frequency availability. In the case of high productivity wells, monthly data may lack the required detail and could be insufficient to estimate reservoir properties with confidence. However, this data frequency may be enough to provide trusty solutions in low permeability wells, where transient flow extends for long times. In any case, daily historic data is desirable for production analysis purposes, since more accurate estimates may be generated from high frequency, high quality data (Anderson et al., 2006).

\subsubsection{Quality Control Verification (Correlation and Consistency)}

Through a simple check, it is possible to assess the quality and completeness of the production data by detecting misleading segments that needs to be corrected or discarded to avoid the generation of possible erroneous results-unrepresentative of the real reservoir features (Mattar and Anderson, 2003).

Some of the most common undesirable events and phenomena affecting the dreamed constant flowing conditions-i.e., the required fluid flow theory assumptions-are: 
- Liquid Loading: Due to low production rates of the unconventional gas wells, liquids tend to accumulate downhole, especially at late production times when wells lack enough energy to carry out liquid columns to surface. This phenomenon can be caused by depletion of the reservoir pressure, changes in operating conditions or by an over-dimensioned completion string. As consequence, production rates may abnormally fluctuate or decrease more than expected, and a misleading unitary slope could appear on diagnostic plots (Agnia et al., 2012). The Turner Plot is one of the methods used to identify the likelihood of liquid loading. According to Turner correlation, if the gasrate/critical-Turner-rate ratio is less than 1 , the flow rate is lower than the critical liquid lift velocity and there is a chance of liquid loading (Fekete, 2013b). Another liquid loading diagnostic plot grounded on the same concept is the Coleman plot. Other indications of unstable flowing conditions caused by liquid loading or slugging are abnormal difference between casing and tubing pressure, very noisy production data and fluctuations or sudden decreases in the water-gas ratio.

- Interference Effects: Production analysis techniques assume constant reservoir drainage. Therefore, if interference due to new infill wells is observed, it is recommended to avoid performing production analysis based on historic data preceding that event. In a few words, if the drainage area changes, the reservoir performance changes completely, and future productions should only be forecasted using data acquired under the new prevalent conditions.

- Alteration of reservoir productivity. As mentioned in section 2 (Decline Curve Analysis), DCA methods are grounded on the assumption that reservoir properties remain constant, because ultimate recovery depends on the formation production performance. So, any change in the reservoir productivity (e.g., stimulation jobs, water breakthrough, condensate rings, etc.) immediately disqualifies DCA models as predicting tool for those cases. In the case of multi-fractured horizontal wells, the most common completion technique in unconventional plays, abnormal high water production is 
expected at early times, as a product of fractures clean-up. Consequently, as water production declines until reaching normal values, skin factor varies, as well as reservoir productivity.

- Surface or wellbore reconfiguration. In the same way, any alteration on operating conditions, as well as in completion configuration, affecting the effective flow area and the flowing pressure will have the same effect as discussed in the previous paragraph.

Nonetheless, given the high complexity of the unconventional wells, many other problems may come out depending on each case. Therefore, interpreters must be aware of these undesirable situations and carefully treat each case as unique, always inquiring about the quality of the input data and the hidden reservoir signals in it. These unstable flowing conditions, as well as production and metering problems, are reflected in different ways. Most common effects are:

- Scattered data. It refers to dispersed points slightly deviated from the main central production decline trend (Noisy data) or points placed away from the average production decline trend (Outliers). Some causes of these worthless points are liquid loading, variable operating conditions, production interference, and measurement and allocation problems (Agnia et al., 2012).

- Steep decrease of production rates with the corresponding increase in flowing pressures.

- Change of the production deliverability potential (variation of flowing rates with an uncorrelated flowing pressure response).

- Abnormal flowing rate fluctuations.

- Time periods without recorded data.

- Significant differences between casing and tubing pressures.

- In the case of gas wells, very low flowing pressures may be indication of very low well energy and its inability to efficiently lift bottom-hole liquids (i.e., liquid loading). 
A practical way of identifying data history inconsistencies is by using the next diagnostic plots:

- Flowing pressures vs. rates. To check the inverse correlation between pressures and rates (Pressures should increase when rates decrease, and Pressures should decrease when rates increase).

- Production data (Flowing pressures and rates) vs. time. To detect abnormal events (liquid loading, workovers, operational setbacks and variations on operation conditions, among others).

- Normalized rate vs. time. According to the fluid flow principles, the $q / \Delta p$ ratio should decrease continuously with time. Any increasing trend is a clear indication of potential non-reservoir effects affecting production behavior (Nobakht and Mattar, 2009).

- Fluids ratios (WGR, WOR, CGR and GOR) vs. time. To identify water breakthroughs, liquid loading, cement leakage. WGR is very useful to identify abnormal high water rates after fracturing (fractures clean-up).

- Tubing and casing pressures vs. time. To identify anomalous behavior of fluid columns in the wellbore.

- Turner or Coleman plots. To detect the likelihood of liquid loading.

Anderson et al. (2006) and Ilk et al. (2011) presented a practical guideline for a systematically diagnosis of production data, including important recommendations and description of most common problems, pitfalls and challenges.

\subsubsection{Data Filtering and Correction}

After identifying inconsistent production data with the diagnostic plots, anomalous data must be corrected (if possible) or filtered and removed to avoid misleading results in the following steps (i.e., flow regime identification and production analysis). The data filtering and correction procedure below was followed in this work. 
- Misleading initial data. As mentioned in the previous section, abnormal high water productions are usually presented at early times just after stimulation jobs as a product of fractures clean-up. This effect alters reservoir productivity. In addition, irregular highly fluctuating or increasing oil/gas rates may be presented. Therefore, this data must not be used for production analysis purposes, because outcomes are not representative of the true reservoir performance-i.e., DCA parameters should not be determined from this data.

- Incomplete data segments. Although it is not a mandatory step, interpolation should be intended if: a) The missing segment is relatively short in comparison with the whole production history, b) Trend of surrounding flowing conditions (before and after the event) is stable, c) Well's record doesn't indicate that a workover, stimulation or any other major operation affecting the reservoir performance was executed during that time period. Interpolated data should be in accordance with the natural trend of the analyzed variables (production rate and flowing pressure), and it may not significantly affect the DCA model match. Depending on each case, this step may be done before or after removing outliers, noise smoothing and rate averaging steps.

- Outlier removal and noise smoothing. Scattered data needs to be corrected to discard worthless/distracting points and to make easier the recognition and interpretation of decline patterns. The first step is removing outliers (i.e., those points that look like spikes). Advanced filtering techniques are available in commercial software (e.g., wavelet decomposition and other range filters). However, in this work a simple algorithm was used to identify and filter out those points out of a band threshold (above and below of the average production trend). This band threshold can be adjusted depending on the standard deviation of the production data in a time window around of each data point. The second step is the noise reduction. In this study, the smoothing process was performed by applying the centered moving average method with an " $n$ " exponent equal to 7 . 
- Rate Averaging: Considering that original input data (rates and flowing pressures) is usually provided in a daily format and most of the DCA applications are designed to work with monthly data (As production volumes are commonly published on this format), a time format conversion may be required for DCA purposes. In contrary, production data on a daily format is strongly advised for RTA purposes. Converting the daily production format to a monthly basis should be done with special care, especially if the well has been inactive during some days of the month. So, rate averaging must account for those discontinuities to avoid unrealistic lower monthly production rates (Agnia et al., 2012). As pointed out by Mattar and Anderson (2003) "The quality of the results may decrease significantly with the degree of averaging performed".

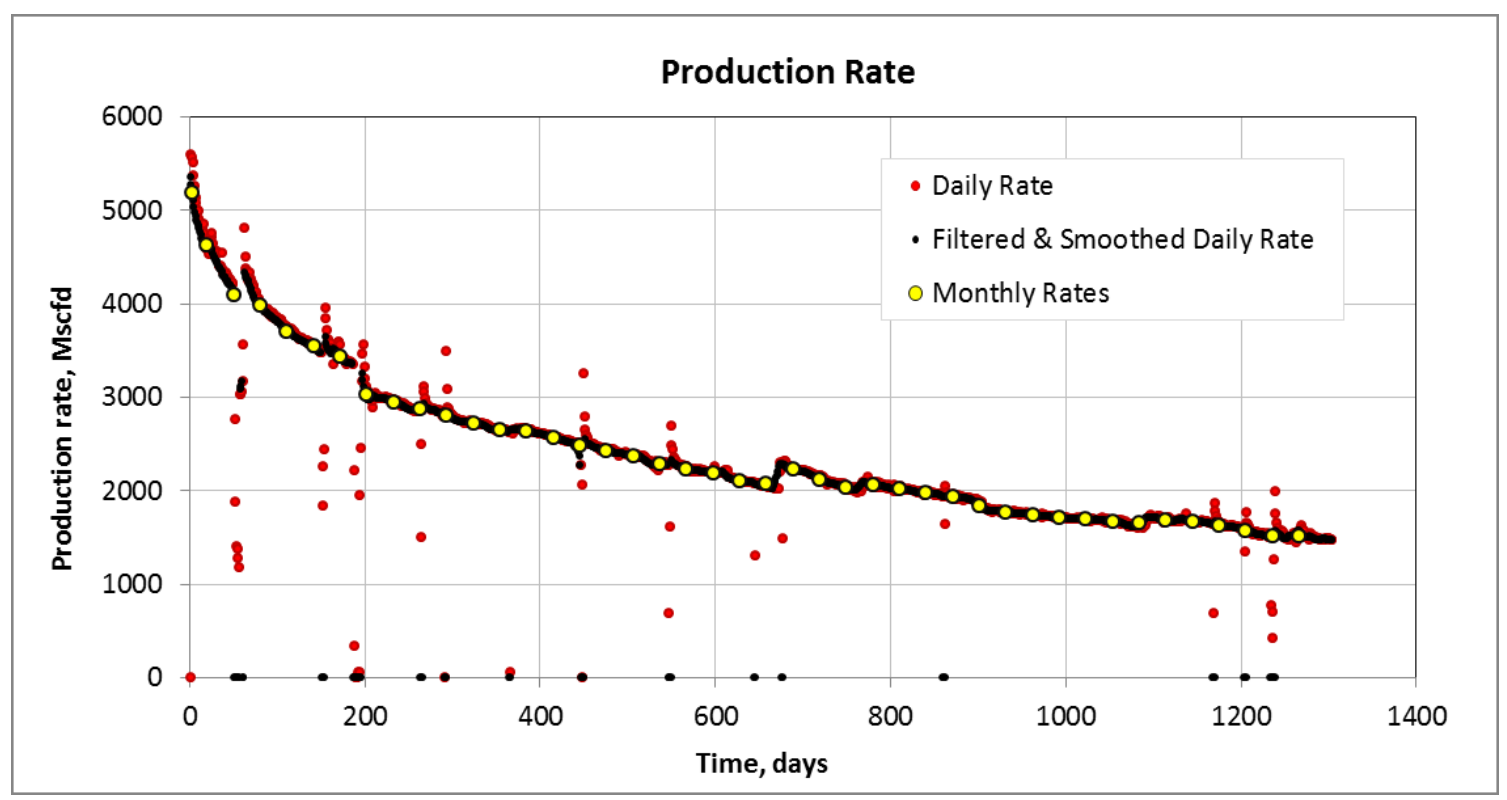

Fig. 33-Correction of production rates from a shale gas well.

Fig. 33 presents an example of a shale gas well where production rates were filtered and converted from a daily to a monthly basis format. Red points correspond to original raw 
data, black points are filtered and smothered data in a daily format, and yellow points are final production rates (corrected and presented in a monthly format, and ready for production analysis).

\subsubsection{Flow Regime Identification}

A complex sequence of different flow regimes may be developed by multi-fractured horizontal wells in ultra-low permeability wells (See Section 3.2.1 for more details). However, the most common flow regimes are transient (linear) and boundary-dominated flow; transient flow is the dominant flow regime. For this reason, modern DCA models specially designed for transient (linear) flow have been advocated. Moreover, dual models-composite models with the particular ability to switch from linear to boundary-dominated flow models at an appropriate time-have been introduced recently (Joshi and Lee, 2013). In practice, decline curve analysis is carried out regardless of the existing flow regimes. As mentioned in Section 4.3.4, inaccurate production forecasts and reserve estimations may be obtained if decline curve analyses are performed without a previous identification of flow regimes (FRI). Each DCA model relies on specific assumptions about flow regimes. Hence, an adequate FRI results in a better understanding of DCA models' limitations, ensure consistent analyses, offers confidence on estimations, and decreases uncertainty associated with DCA forecasts (Mangha et al., 2012). Taking into account these reasons, flow regime identification is considered a key step of this methodology.

A comprehensive explanation of most important flow regime identification techniques is presented in Section 3.2.2. In this methodology, derivative plots have not been included Table 8 describes the diagnostic plots used in this study for flow regime identification purposes.

Two different flow regime identification approaches are presented in this methodology. The first approach-single method approach-is grounded in the consideration that flow regimes can be identified with confidence by applying a single optimized method, which has the special ability of overcoming most of the inconsistencies that usually affect diagnosis 
processes. The second approach-multi-method approach-relies on the concept that a combined analysis applying all the diagnosis methods may generate more reliable estimations.

Table 8-Diagnostic plots for flow regime identification

\begin{tabular}{|c|c|c|c|}
\hline \multicolumn{2}{|c|}{ Diagnostic Plots } & Flow Regimes & Description \\
\hline 1 & $\log q-\log t$ & Bilinear, Linear, and BDF & Logarithmic plot of production rate vs. real time \\
\hline 2 & Log q - Log MBT & Bilinear, Linear, and BDF & Logarithmic plot of production rate vs. MBT \\
\hline 3 & $\log \mathrm{q} / \triangle \mathrm{P}-\log \mathrm{MBT}$ & Bilinear, Linear, and BDF & Logarithmic plot of PNR vs. MBT \\
\hline 4 & $\log q / \Delta \Psi-\log T c a$ & Bilinear, Linear, and BDF & Logarithmic plot of PNR vs. material balance pseudotime \\
\hline 5 & $\Delta P / q-S R(T c)$ & Linear and BDF & Cartesian plot of RNP vs. square root of MBT \\
\hline 6 & $\Delta \Psi / \mathrm{q}-\mathrm{SR}(\mathrm{Tca})$ & Linear and BDF & $\begin{array}{l}\text { Cartesian plot of rate-normalized pseudopressure vs. square } \\
\text { root of material balance pseudotime }\end{array}$ \\
\hline 7 & $\Delta P / q-F R(T c)$ & Bilinear & Cartesian plot of RNP vs. fourth root of MBT \\
\hline 8 & $\Delta \Psi / \mathrm{q}-\mathrm{FR}(\mathrm{Tca})$ & Bilinear & $\begin{array}{l}\text { Cartesian plot of rate-normalized pseudopressure vs. fourth root } \\
\text { of material balance pseudotime }\end{array}$ \\
\hline
\end{tabular}

Multi-fractured horizontal wells are the most common completion technique in unconventional plays, as mentioned in section 5.1.1.2. Therefore, abnormal high water production may be presented at early times due to fractures clean-up. In this process, water production rates tend to decrease until reaching normal values, and as a result, formation skin factor changes. Thus, misleading bilinear flow signatures may appear as consequence of the changing skin effect. In practice, no data prior to linear flow should be used to determine parameters for any decline model, in order to avoid misinterpretations. 


\subsubsection{Single Method Approach}

The first four methods (Log-Log plots) are considered as the preferred flow regime identification method. In practice, all these plots are based on the same concept (Bilinear Flow: -1/4; Linear Flow: -1/2; BDF: -1). However, plot variables are different on each case. The first plot ( $\log q-\log t)$ is the most common flow regime identification plot used by interpreters. But, as this plot relies only on production rates and real time, flow regimes shouldn't be diagnosed based on its outputs. The second plot (Log q - Log MBT) includes material balance time, instead of real time, which represents an improved analysis capacity for wells flowing under unstable operating conditions (See Section 3.1.1). Third and fourth plots (Log $q / \Delta P$ - Log $M B T$, Log $q / \Delta \Psi$ - Log Tca) are recommended as the best option to identify with confidence flow regimes, because they both are based on PNR, and real time has been replaced by the material balance time function. Taking into account these considerations, these two plots should be used as main diagnostic plots. As the fourth one ( $\log q / \Delta \Psi$ - Log Tca) includes pseudo-variables (pseudopressure and material balance pseudotime), it is recommended for gas cases. The third plot (Log $\mathrm{q} / \Delta \mathrm{P}$ - Log $\mathrm{MBT}$ ) is used for oil cases mainly. Advantages of pseudovariables are described in Section 3.1.2.

\subsubsection{Multi-Method Approach}

This approach considers that a single diagnostic plot is not enough to identify flow regimes with confidence. Instead, a combined perspective may provide a better insight and reduce the uncertainty associated to a method in particular. Therefore, the final determination about flow patterns occurrence is the product of different methods. However, as some diagnostic methods offer special advantages over others, no all the single solutions should be treated equitably. Thus, single methods are prioritized and final solution is estimated as a weighted average of all single solutions. Eq. 49 shows the expression for the probability of occurrence of a flow regime. $M_{i}$ represents each method's output (1 means that the flow regime has been positively identified by that method in particular; 0 means the opposite. Intermediate values may also be used to express specific uncertainties). $W_{i}$ is the weight assigned to each method and express how confident interpreters are with each method in 
particular. Additionally, an optional reliability factor $\left(\mathrm{R}_{\mathrm{RF}}\right)$ is included to account for the confidence that interpreters have in quality of input data. Finally, wells may be classified according to the probability of occurrence of each flow regime $[P(F R)]$, as presented below. Presenting FRI results in probabilistic terms is an advantage because in most cases, it is difficult to affirm with certainty that a flow regime has occurred, especially when production data quality is questionable.

$$
P(F R)=R_{F R} * \sum_{i=1}^{n}\left(M_{i} * W_{i}\right)
$$

- $\quad$ No evidence: $P(F R)=0 \%$

- Possible: $\quad \mathrm{P}(\mathrm{FR})<30 \%$

- Probable: $30 \% \leq \mathrm{P}(\mathrm{FR})<70 \%$

- Very likely: $\quad P(F R) \geq 70 \%$

The specialized bilinear and linear flow regimes identification plots are recommended to be included in this approach along with Log-Log methods (See plots 5 through 8 in Table 8). The concept of those plots is extensively explained in Sections 3.2.5 and 3.2.4, respectively.

\subsubsection{Hindcasting Analysis}

This is a testing method used to validate mathematical models by comparing model's outputs with known historic data from past events. For production analysis purposes, hindcasts offer the possibility of measuring the relative error of results provided by a forecasting model in relation with the real production performance. Considering the purpose of this study, hindcasts will be used to evaluate the applicability and the accuracy of pressure-normalized decline curve analysis (PN-DCA) as a production forecasting technique.

Although this step is not required to analyze production data, it is advised to perform a hindcasting analysis to tune/calibrate the forecasting analysis methodology (selecting adequate DCA models and techniques) before estimating future production and recoveries. In any case, it may help interpreters to better understand prediction models and to reduce uncertainty 
associated to estimations. Wells production performance depends on multiple fluid and reservoir features. Given the high heterogeneity of these parameters in any play, wells exhibit particular production behaviors. Therefore, DCA models effectiveness may vary depending on the particular features of each case. While certain DCA models achieve outstanding results in some plays, the same models may not work very well in other cases, even if there is a high level of similarity.

\subsubsection{Forecasting Analysis}

This step corresponds to the application of decline curve analysis techniques to forecast future production performance and to estimate remaining reserves. This study is focused on the application of the pressure-normalized decline curve analysis (PN-DCA) techniques, which consist of performing decline curve analysis (DCA) using pressure-normalized rates (PNR) instead of real production rates. In this work, eight DCA models (Arps, Modified Hyperbolic, SEPD, MSE, Duong, Modified Duong, PLE and LGM) and five pressure normalization techniques have been implemented (PN, $\triangle \Psi N, S P N, \triangle P F$, and $H E$ ). A comprehensive description of DCA models is presented in Section 2. Additional details about the transformation of real production rates into pressure-normalized rates, and the application of PN-DCA techniques are described in Section 4.4.

As mentioned in Section 2.2, each DCA model relies on specific flow regimes. Arps models assume BDF, modern DCA models (SEPD, Duong, Logistic Growth, and Power Law Exponential) are based on linear flow, and dual models are specially designed to switch from an early transient model to a terminal BDF model when a limiting condition is reached (switching time or a terminal decline rate). Ideally, this limiting condition should correspond to the BDF onset. However, if BDF conditions have not been reached, which is the most common case in UCR's, reasonable limiting conditions should be estimated by the interpreter. If the BDF signature is identified in the flow regime analysis, production forecast should be performed using just the BDF portion of the production history with a BDF model (if that portion is enough to match the model with confidence). In this work three dual models have been included in the analysis (Modified Hyperbolic, MSE, and Modified Duong). If BDF is not observed, a minimum 
decline rate of $10 \% / y r$ has been assumed, and the terminal decline model is Arps' Hyperbolic with $\mathrm{a} b$ factor of 0.3 for oil wells and 0.4 for gas wells.

Along this work, the traditional DCA technique (based on production rates only) and the novel pressure normalization techniques (based on PNR) will be all referred as DCA techniques. Decline curve analysis was developed using PDCA, an application created by the Unconventional Reservoir Research Group of Texas A\&M University to perform deterministic and probabilistic decline curve analysis.

\subsection{Evaluation of Pressure-Normalized Decline Curve Analysis (PN-DCA) as Production Forecasting Technique}

The principal purpose of this section is to assess the feasibility of implementing decline curve analysis based on pressure-normalized rates (PN-DCA) as a reliable production forecasting technique. The best way to evaluate the accuracy of this new method is through hindcasting analyses (see Section 5.1.1), where predictions generated by PN-DCA methods are compared with real cumulative production data. In order to obtain more representative results, this process will be applied on multiple wells. In addition, several comparisons will be done considering different conditions, in order to identify under which specific conditions PN-DCA techniques may produce more accurate results, if possible.

\subsubsection{Production Data Diagnosis and Preparation}

The representativeness of this comparative study depends on the quality and amount of production data available for the analysis. Indeed, the main restriction of this work was obtaining real and reliable production data. As mentioned in Section 5.1.1.1, a large amount of well data is required to apply PN-DCA techniques, especially if bottomhole pressure measurements are not available. As most of this information is considered confidential by operator companies, it is extremely difficult to have access to the required whole set of well data. Nevertheless, some companies agreed to provide this information with the commitment 
of keeping confidential the source and some specific details of each well (e.g., name and location). Therefore, wells names have been modified, and specific well parameters are not shown in this work.

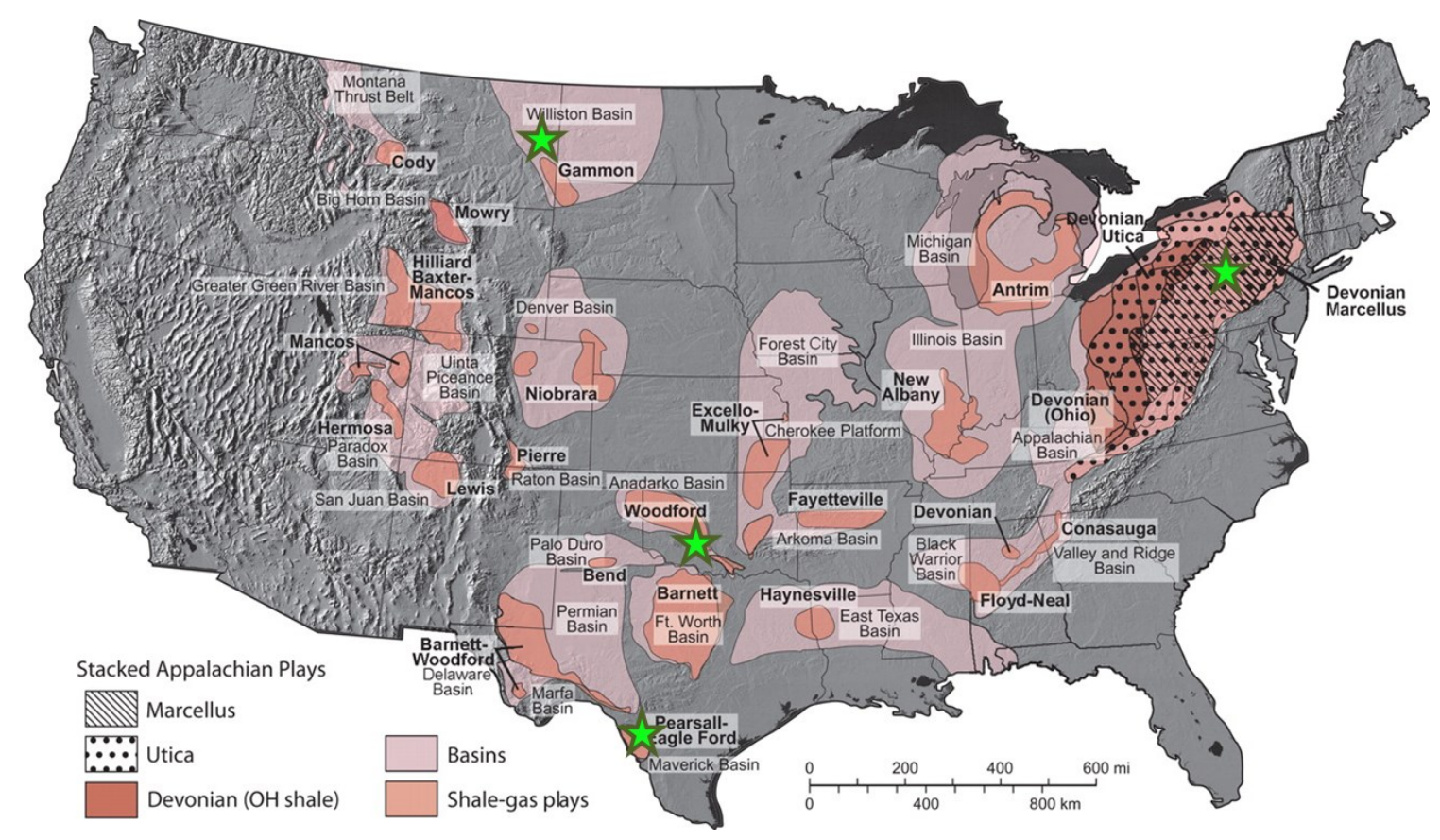

Fig. 34-Location of unconventional wells used in this study. After Gale and Holder (2010).

In total, production data of 47 wells, located in four different unconventional plays (Marcellus, Cana Woodford, Eagle Ford and Bakken), was provided by different operators. 31 wells (66\%) are gas producers and 16 wells are oil producers (34\%). Fig. 34 shows a map with the approximate locations of these wells. Table 9 presents a summary of these wells, including some features like type of fluid, production history, data quality and liquid loading. Production rates and pressures were provided in a daily basis format. This format was preserved for flow regime identification purposes, but it was converted to a monthly format for the application of PN-DCA techniques. Bottomhole flowing pressure (BHP) measurements were not available. 
Therefore, tubing head pressures (THP) were converted to sandface conditions (BHP) using Fekete Harmony suite.

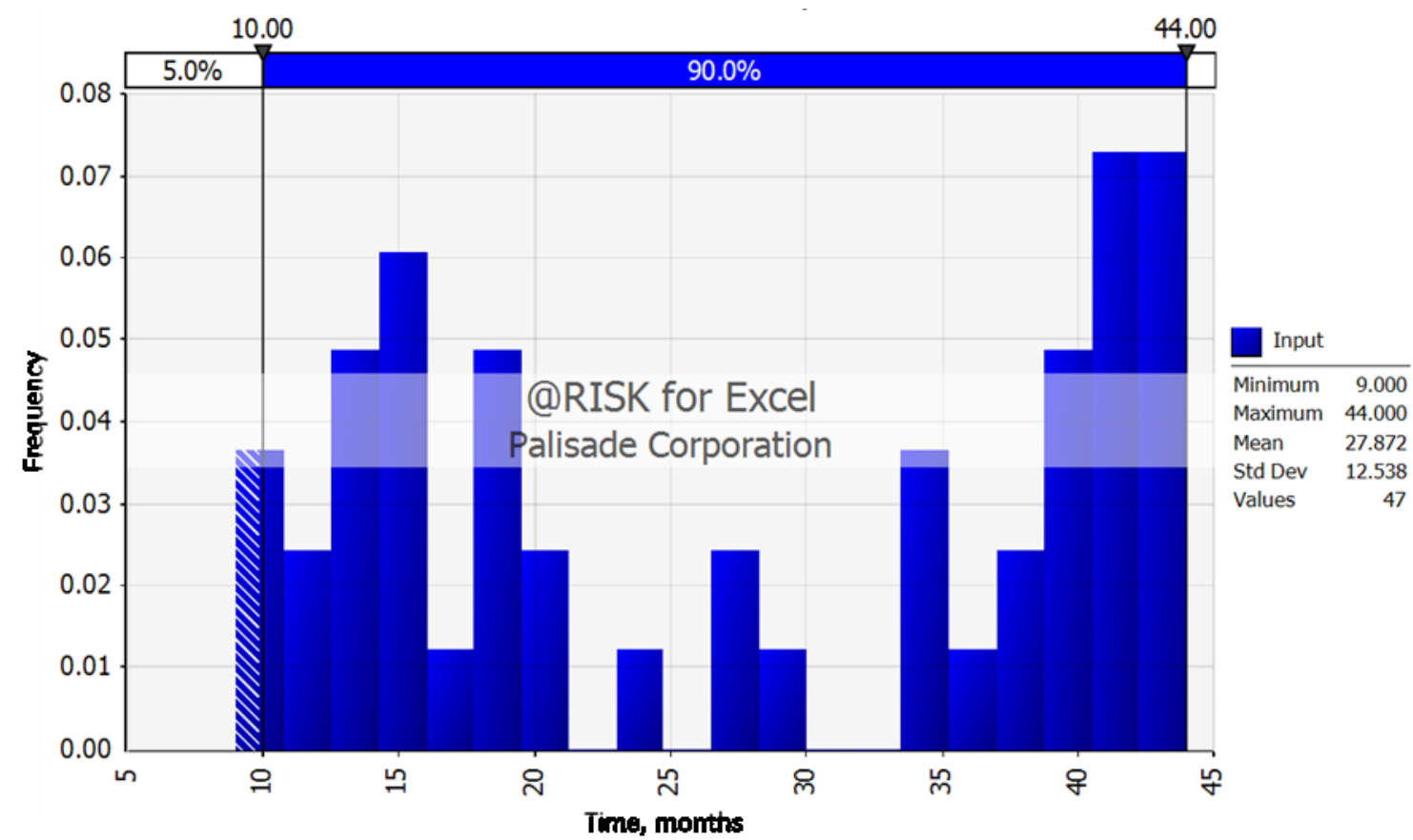

Fig. 35-Histogram of the production histories duration for all wells.

As unconventional wells are expected to produce for long times, it is necessary that forecasting models have the ability of accurately predict production performance over long periods. Therefore, another important factor that has a big impact on the representativeness of this study is the duration of production histories. The longer the production history, the more representative the results. Fig. $\mathbf{3 5}$ shows a histogram of the duration of production histories for all wells included in this study. Fig. 36 presents a comparative illustration of the duration (minimum, average, maximum) of production history of each play. Input data quality is also another important issue. 
Table 9-List of wells used for the hindcasting analysis

\begin{tabular}{|c|c|c|c|c|c|c|c|c|}
\hline \multirow{3}{*}{ Play } & \multirow{3}{*}{ Well } & \multirow{2}{*}{ Fluid } & \multicolumn{2}{|c|}{ Production History } & \multicolumn{3}{|c|}{ Data Quality } & \multirow{2}{*}{$\begin{array}{c}\begin{array}{c}\text { Liquid } \\
\text { Loading }\end{array} \\
\text { Turner Plot }\end{array}$} \\
\hline & & & Days & Months & B & $\mathbf{R}$ & G & \\
\hline & & G/O & No. & No. & $x$ & $x$ & $x$ & PLL/FLL \\
\hline \multirow{14}{*}{ Marcellus } & M-1 & G & 1288 & 43 & & $x$ & & $60 \%$ \\
\hline & $\mathrm{M}-2$ & G & 1187 & 40 & & $x$ & & $20 \%$ \\
\hline & $M-3$ & G & 1188 & 40 & & $x$ & & $0 \%$ \\
\hline & $\mathrm{M}-4$ & G & 1285 & 43 & & $x$ & & $100 \%$ \\
\hline & M-5 & G & 1234 & 41 & & $x$ & & $0 \%$ \\
\hline & M-6 & G & 1254 & 42 & $x$ & & & $60 \%$ \\
\hline & $\mathrm{M}-7$ & G & 1254 & 42 & $\mathrm{x}$ & & & $81 \%$ \\
\hline & M-8 & G & 1226 & 41 & & $x$ & & $0 \%$ \\
\hline & M-9 & G & 1135 & 38 & & $x$ & & $0 \%$ \\
\hline & $\mathrm{M}-10$ & G & 1156 & 39 & & $x$ & & $29 \%$ \\
\hline & $\mathrm{M}-11$ & G & 1017 & 34 & & $x$ & & $0 \%$ \\
\hline & $\mathrm{M}-12$ & G & 1226 & 41 & & $x$ & & $29 \%$ \\
\hline & $\mathrm{M}-13$ & G & 1161 & 39 & & $x$ & & $20 \%$ \\
\hline & $\mathrm{M}-14$ & $\mathrm{G}$ & 1016 & 34 & & $x$ & & $0 \%$ \\
\hline \multirow{12}{*}{ Woodford } & WF-1 & G & 1304 & 44 & & & $\mathrm{x}$ & $100 \%$ \\
\hline & WF-2 & G & 793 & 27 & & & $\mathrm{x}$ & $100 \%$ \\
\hline & WF-3 & G & 1138 & 38 & & & $\mathrm{x}$ & $100 \%$ \\
\hline & WF-4 & G & 1314 & 44 & & & $\mathrm{X}$ & $100 \%$ \\
\hline & WF-5 & G & 1315 & 44 & & & $\mathrm{x}$ & $100 \%$ \\
\hline & WF-6 & G & 1289 & 43 & & $x$ & & $100 \%$ \\
\hline & WF-7 & G & 570 & 19 & & & $\mathrm{X}$ & $100 \%$ \\
\hline & WF-8 & G & 565 & 19 & & & $\mathrm{X}$ & $100 \%$ \\
\hline & WF-9 & G & 826 & 28 & & & $\mathrm{X}$ & $100 \%$ \\
\hline & WF-10 & G & 1112 & 37 & & & $\mathrm{X}$ & $100 \%$ \\
\hline & WF-11 & G & 1265 & 42 & & & $\mathrm{X}$ & $100 \%$ \\
\hline & WF-12 & $\mathrm{G}$ & 1048 & 35 & & $x$ & & $100 \%$ \\
\hline \multirow{16}{*}{ Eagle Ford } & EF-1 & $\mathrm{O}$ & 437 & 17 & $x$ & & & -- \\
\hline & $E F-2$ & 0 & 421 & 16 & $x$ & & & -- \\
\hline & EF-3 & $\mathrm{O}$ & 414 & 15 & & & $x$ & -- \\
\hline & $E F-4$ & G & 310 & 11 & & $x$ & & $100 \%$ \\
\hline & $E F-5$ & G & 675 & 24 & $X$ & & & $100 \%$ \\
\hline & EF-6 & 0 & 565 & 21 & & $x$ & & -- \\
\hline & EF-7 & 0 & 420 & 16 & $x$ & & & -- \\
\hline & $E F-8$ & 0 & 415 & 16 & & $x$ & & -- \\
\hline & EF-9 & 0 & 259 & 10 & $x$ & & & -- \\
\hline & EF-10 & 0 & 241 & 9 & $x$ & & & -- \\
\hline & EF-30 & 0 & 247 & 9 & & & $x$ & -- \\
\hline & EF-31 & G & 467 & 16 & $x$ & & & $80 \%$ \\
\hline & $E F-40$ & G & 609 & 21 & & & $x$ & $0 \%$ \\
\hline & $E F-41$ & 0 & 371 & 13 & $x$ & & & -- \\
\hline & EF-42 & 0 & 556 & 19 & & & $x$ & -- \\
\hline & EF-43 & 0 & 397 & 14 & & $x$ & & -- \\
\hline \multirow{5}{*}{ Bakken } & BK-1 & 0 & 382 & 14 & & $x$ & & -- \\
\hline & BK-2 & 0 & 382 & 14 & & & $x$ & -- \\
\hline & BK-3 & 0 & 291 & 11 & & $x$ & & -- \\
\hline & BK-4 & G & 531 & 18 & $x$ & & & $100 \%$ \\
\hline & BK-20 & 0 & 854 & 29 & & $x$ & & -- \\
\hline
\end{tabular}




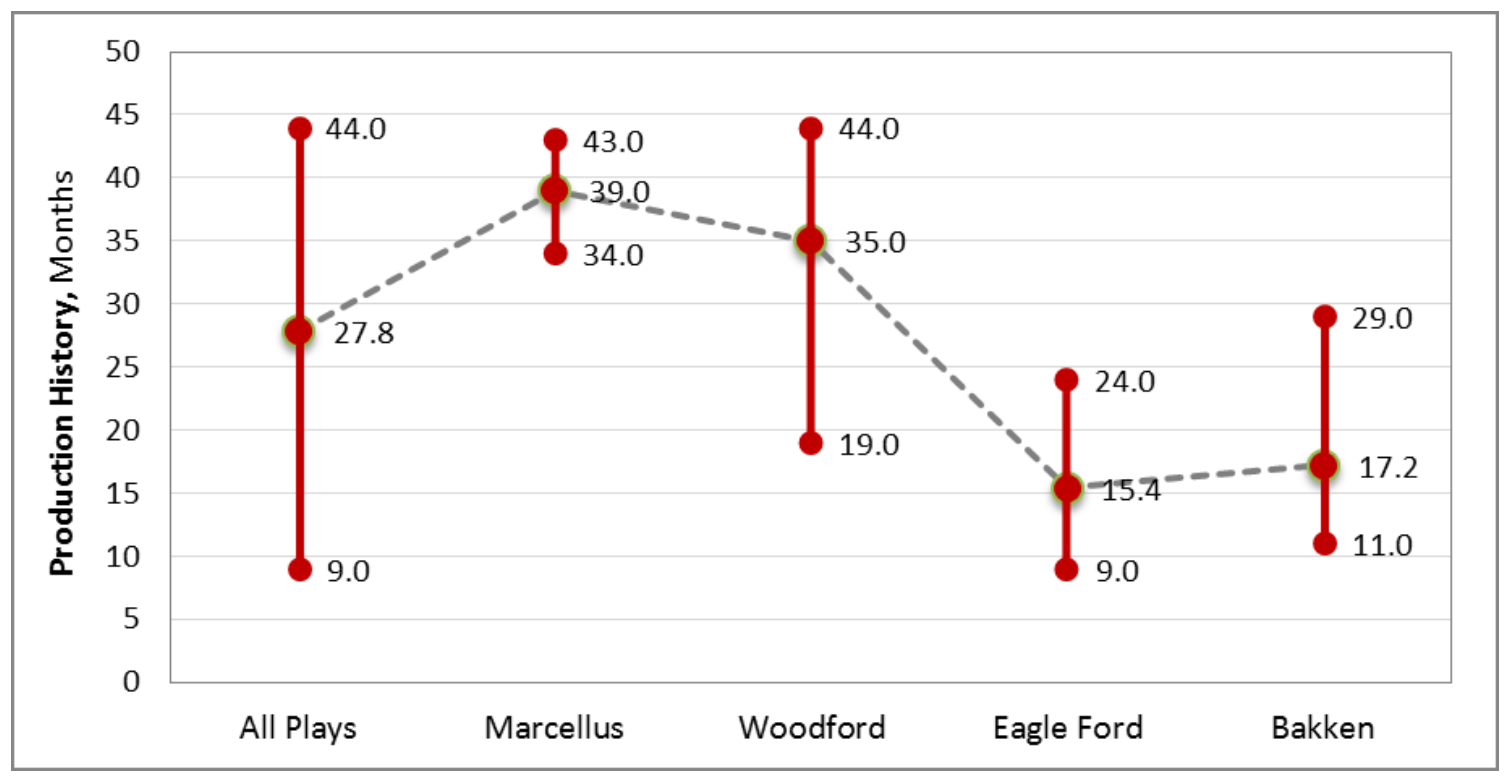

Fig. 36-Production history times (minimum, mean and maximum) for each play.

Quality control has been verified by following indications presented in Section 5.1.1.2. Table 9 includes a data quality column showing qualification assigned to each well depending on the accomplishment of these criteria (B: bad, R: regular, G: good). Bad quality means that well's production performance has been severely affected by strong fluctuations in operational conditions, frequent discontinuities and multiple outliers appear during production history, matching decline models with confidence is difficult, and flowing pressures may not correlate with production rates. In general, there is a high uncertainty associated with predictions derived from this kind of data. Regular quality refers to minor problems that don't affect significantly production forecasts. Minor problems include few outliers, slightly scattered data and short discontinuities that don't deform the natural decline profile. In general, production rates correlate well with flowing pressures, and it is easy to apply DCA models with confidence. In opposite, good quality means that most of production data is not severely affected by the previously mentioned problems. Note that major and long-lasting changes in operating conditions (e.g., increase of production rates caused by an intentional reduction of tubing head pressures) are not considered a quality problem. In all cases, operators have not reported or provided information about major operations affecting reservoir productivity features. Thus, it 
is assumed that there are not restrictions to apply DCA (See Section 5.1.1.2). Main observations about data quality, production history and other specific details of each play are discussed below.

\subsubsection{Marcellus}

This study includes 14 gas wells from Marcellus shale with an average production history of 39 months (See Fig. 36). Fig. 37 shows original production rates and flowing pressures of well M-1. Fig. 38 presents production rates in both daily and monthly format, after being processed (i.e., data filtering and correction), as described in Section 5.1.1.3.

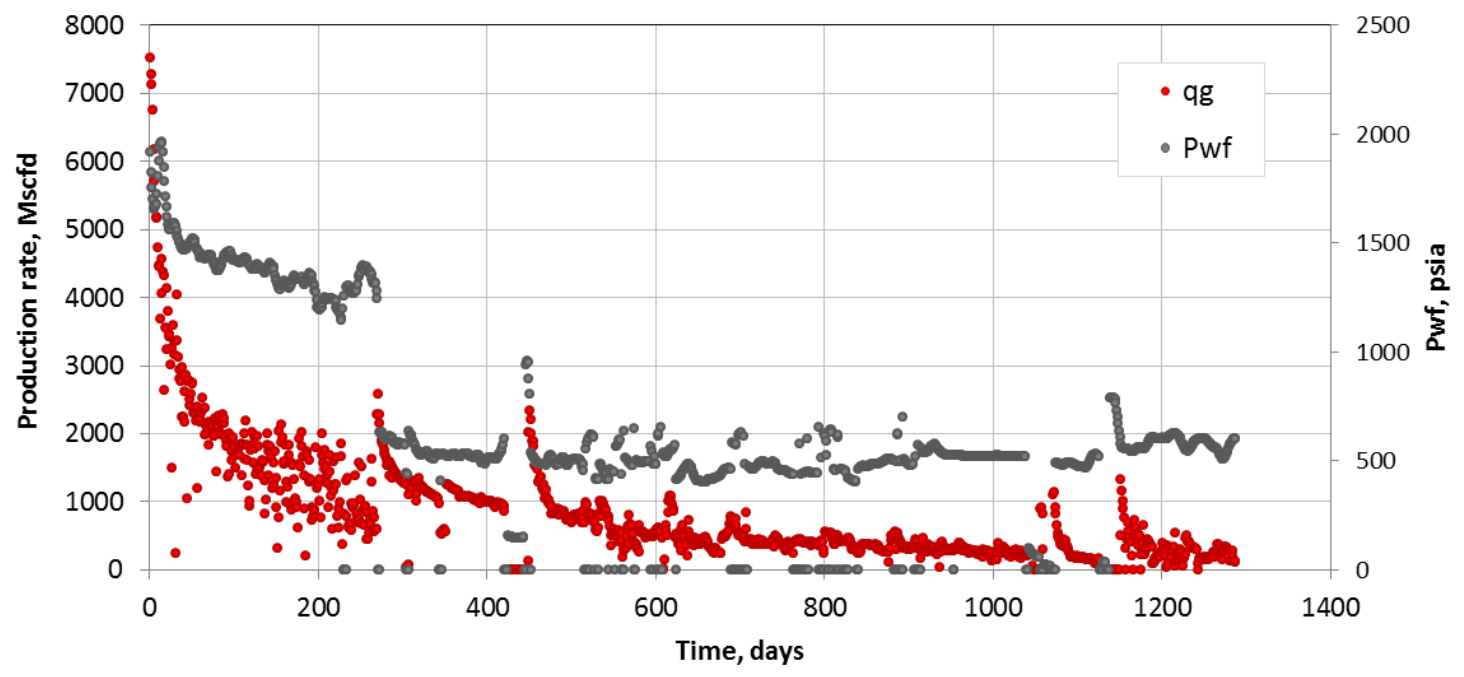

Fig. 37-Production rates and flowing pressures of $\mathrm{M}-1$ well.

In general, average quality of production data is regular, excepting 2 wells that were classified as bad quality. This means that most of wells exhibit similar minor problems that don't significantly affect production forecasts, as previously described. Only 6 wells don't exhibit liquid loading problems. Four wells present minor problems of liquid loading $(<30 \%$ of production history), while 4 wells show liquid loading throughout the majority of their productive life. Most times, liquid loading problems are observed at late times, when production rates have decreased significantly. However, no major fluctuations or 
inconsistencies due to liquid loading were observed. Although it doesn't represent a significant problem for decline curve analysis, flow regime diagnosis may be affected. This issue will be discussed in Section 5.2.2. Fig. 39 shows the liquid loading diagnostic analysis of well M-1. Note that liquid loading occurs during around $60 \%$ of well life. Initial reservoir pressures range between 3594 and 4029 psi, and the average is 3830 psi (See Fig. 40).

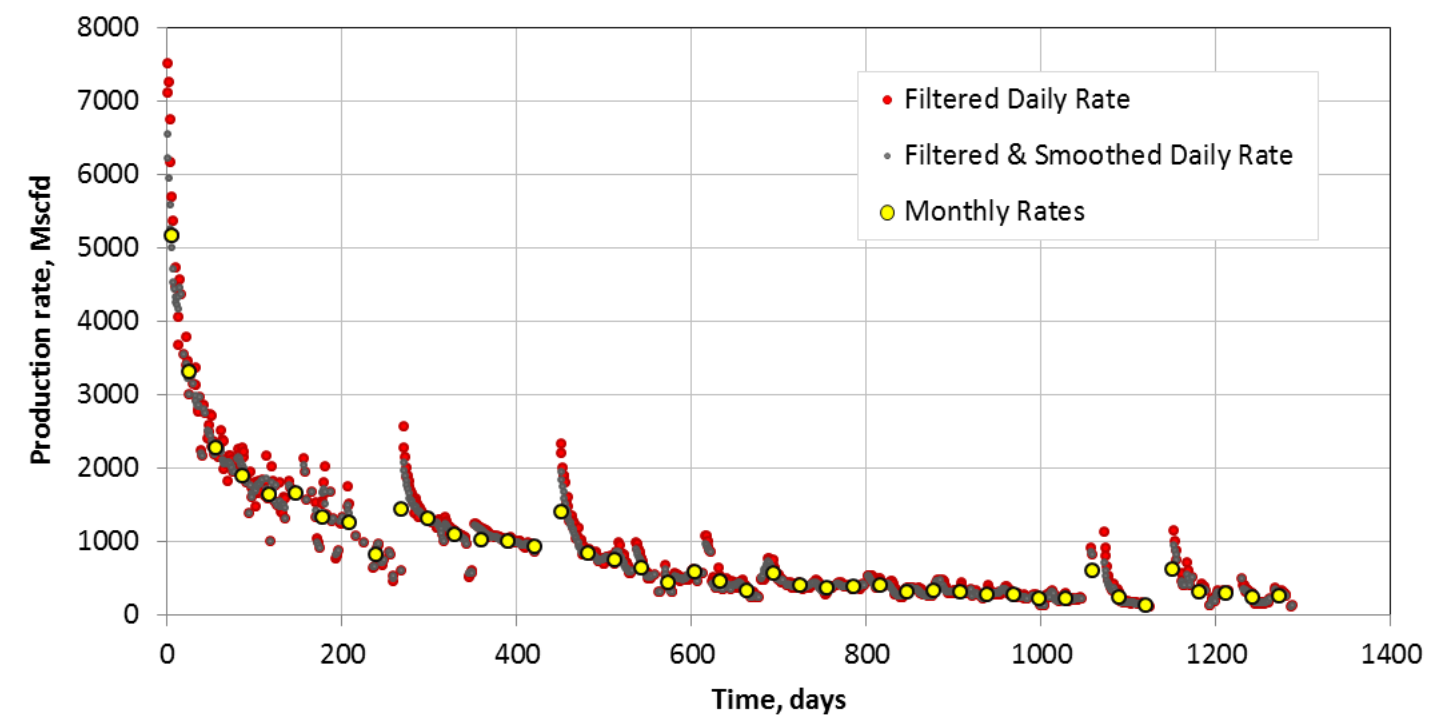

Fig. 38-Production rates of well M-1 presented in daily and monthly format

\subsubsection{Woodford}

This study includes 12 gas wells from Woodford shale with an average production history of 35 months (See Fig. 36 and Table 9). Fig. 41 shows original production rates and flowing pressures for well W-10. In general, the average data quality of these Woodford wells is excellent, excepting 2 wells that were classified as regular. Although all wells present liquid loading problem, it seems not to affect production performance-fluctuations and discontinuities are not observed. Initial reservoir pressures range between 8361 and 9020 psi, and the average is 8694 psi (See Fig. 40). 


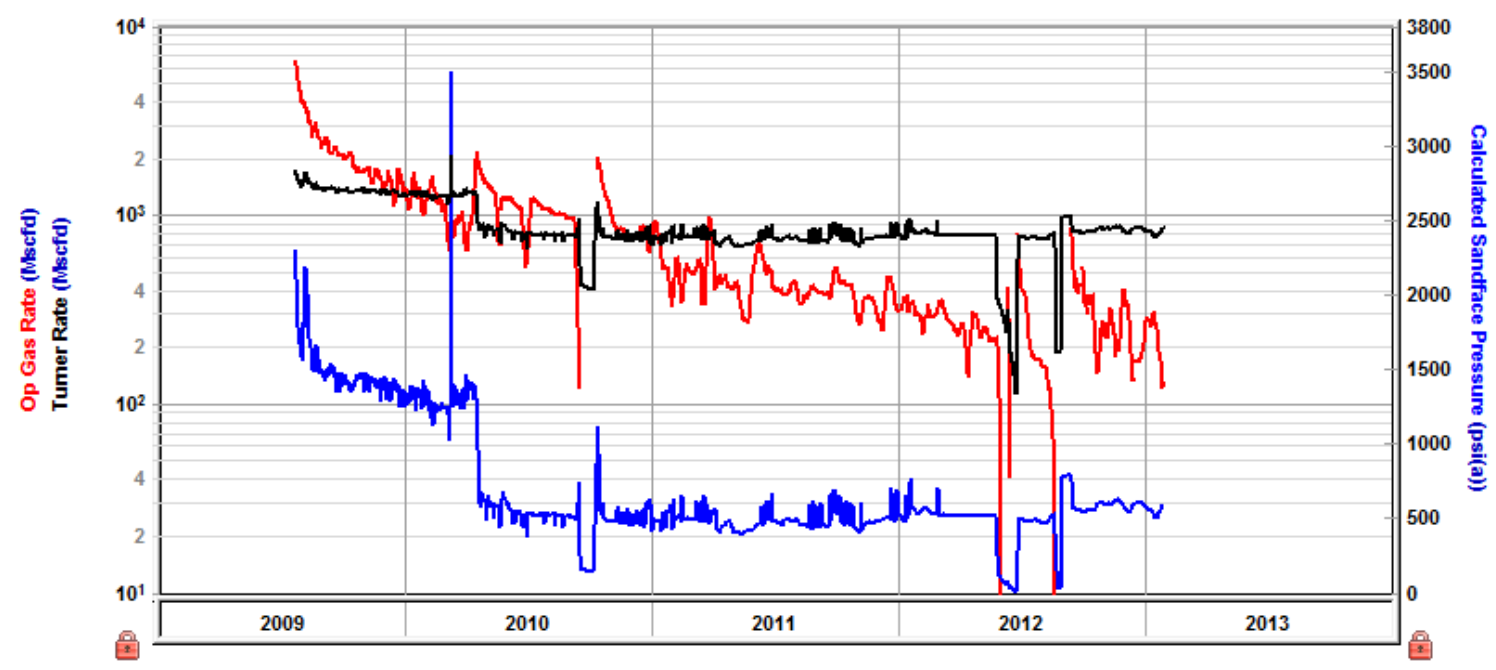

Fig. 39-Liquid loading diagnostic plot (Turner plot) of well M-1

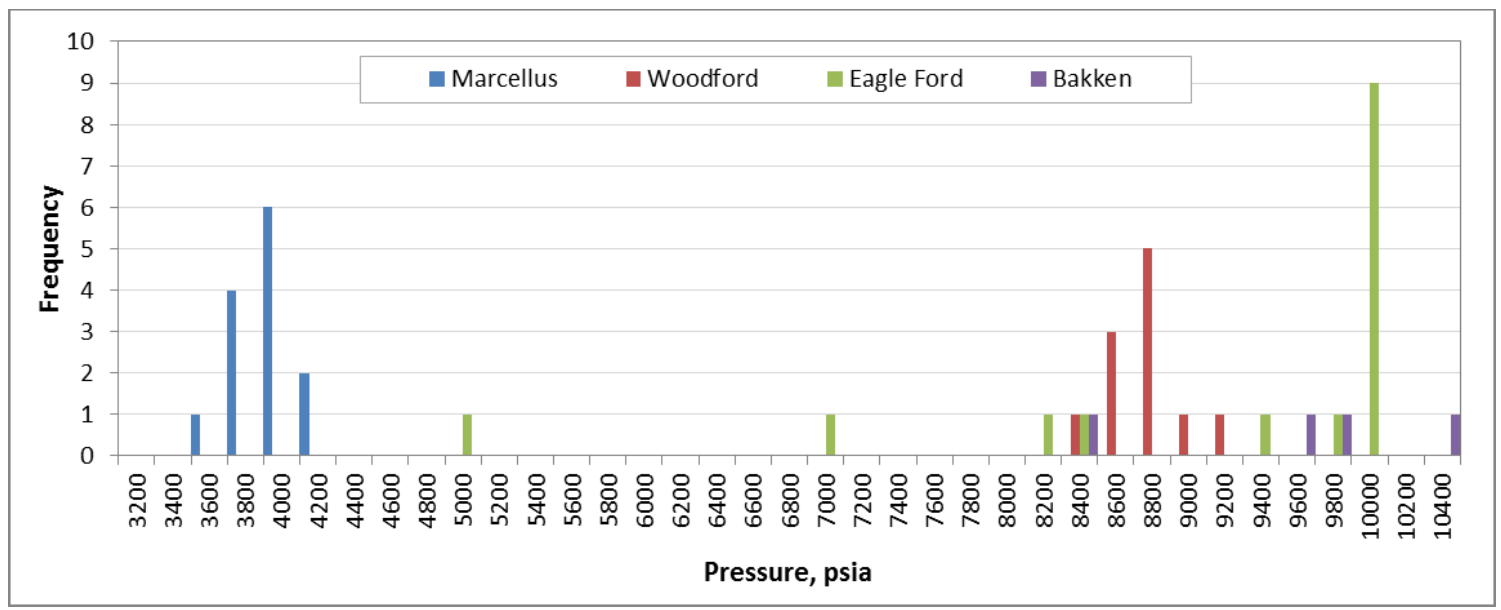

Fig. 40-Histogram of initial reservoir pressures for each play. 


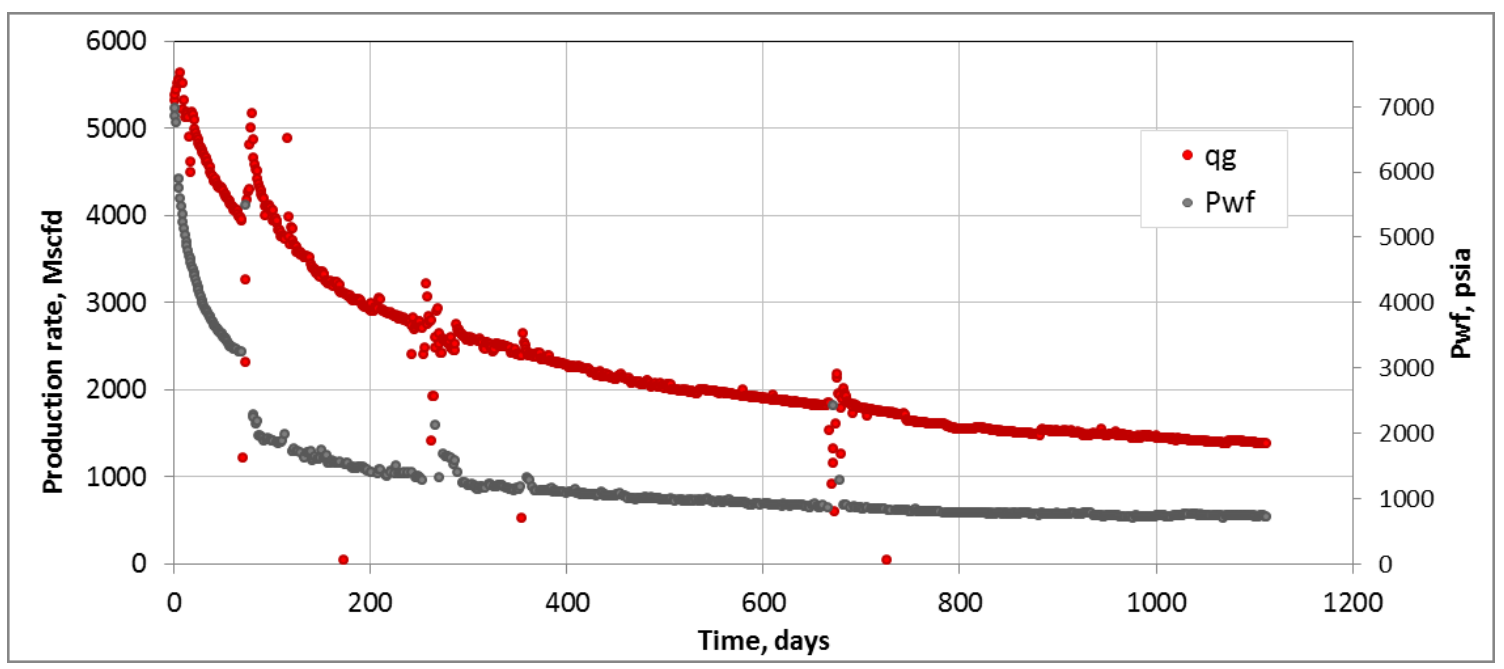

Fig. 41-Production rates and bottomhole flowing pressures of well W-10.

\subsubsection{Eagle Ford}

This study includes 16 wells from Eagle Ford shale with an average production history of 15.4 months (See Fig. 36 and Table 9). There are 12 oil producer and 4 gas producers. Fig. 37 shows original production rates and flowing pressures of well EF-7. In general, production data quality is variable (See Table 9). Most of wells exhibit major problems of discontinuity and dispersion that may significantly affect production forecasts. In addition, three of the 4 gas wells exhibit liquid loading problem. Given these quality problems and the short production history of some wells, five wells were excluded from the hindcasting analysis, although they all are considered in the flow regime identification analysis. Fig. $\mathbf{4 2}$ shows production data of one of these wells. Note how dispersed data is early on and the frequent discontinuities later on. On the other hand, 7 of 16 wells correspond to high pressure, rate controlled wells (See Section 4.3.2). Fig. 43 shows an example of a high pressure, rate restricted well. Note that average production rates practically don't decline during the first year (black line), while bottomhole flowing pressures slowly decline. Initial reservoir pressures range between 5000 and 10000 psi, and the average is 9198 psi (See Fig. 40). Most of the wells are in the high pressure area (initial reservoir pressure: $10.000 \mathrm{psi})$. 


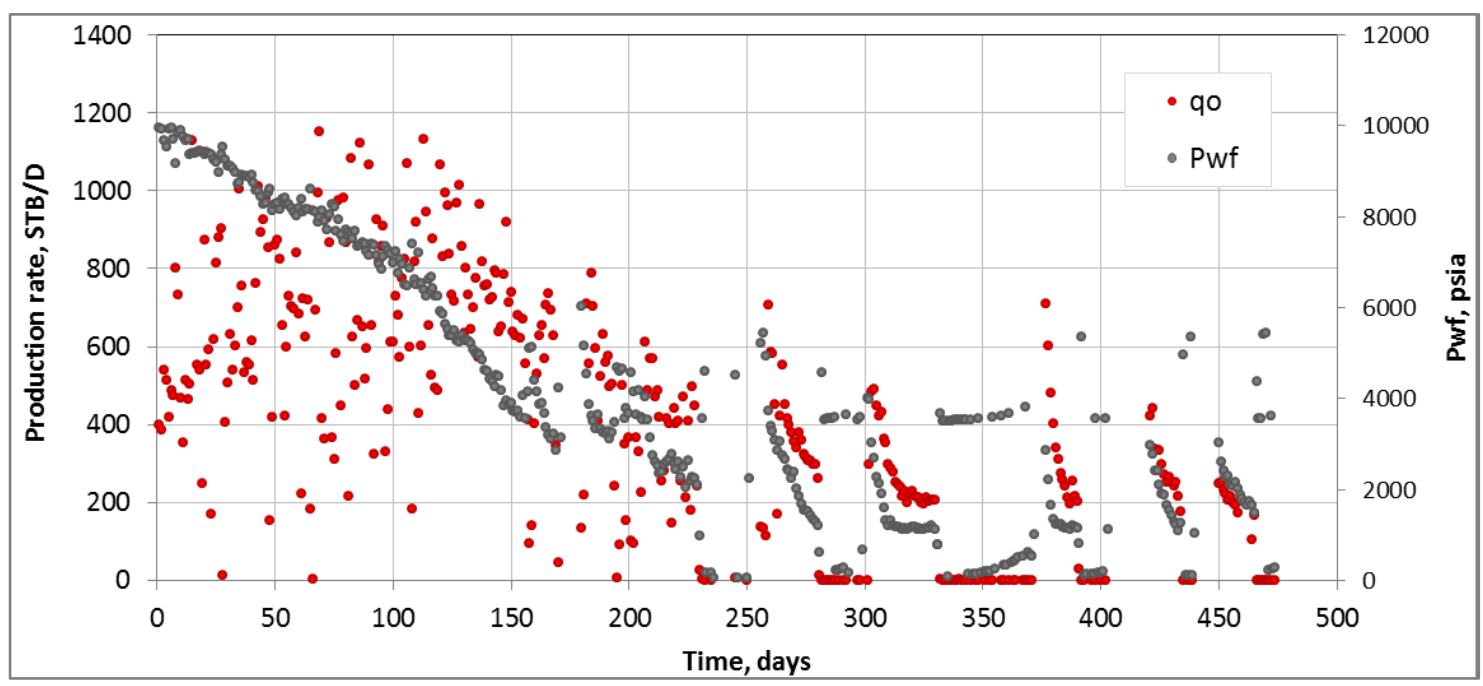

Fig. 42-Production rates and flowing pressures of well EF-7.



Fig. 43-High pressure, rate controlled Eagle Ford well (EF-2).

\subsubsection{Bakken}

This study includes only 5 wells from Bakken shale with an average production history of 17.2 months (See Fig. 36 and Table 9). There are 4 oil producer and 1 gas producer. Fig. 44 
shows original production rates and flowing pressures for well BK-2. In general, production data quality is variable, just like Eagle Ford wells (See Table 9). However, unlike Eagle Ford wells, the most common problem is high data scattering, which may not significantly affect production forecasts. Initial reservoir pressures range between 8300 and 10226 psi, and the average is 9278 psi (See Fig. 40).

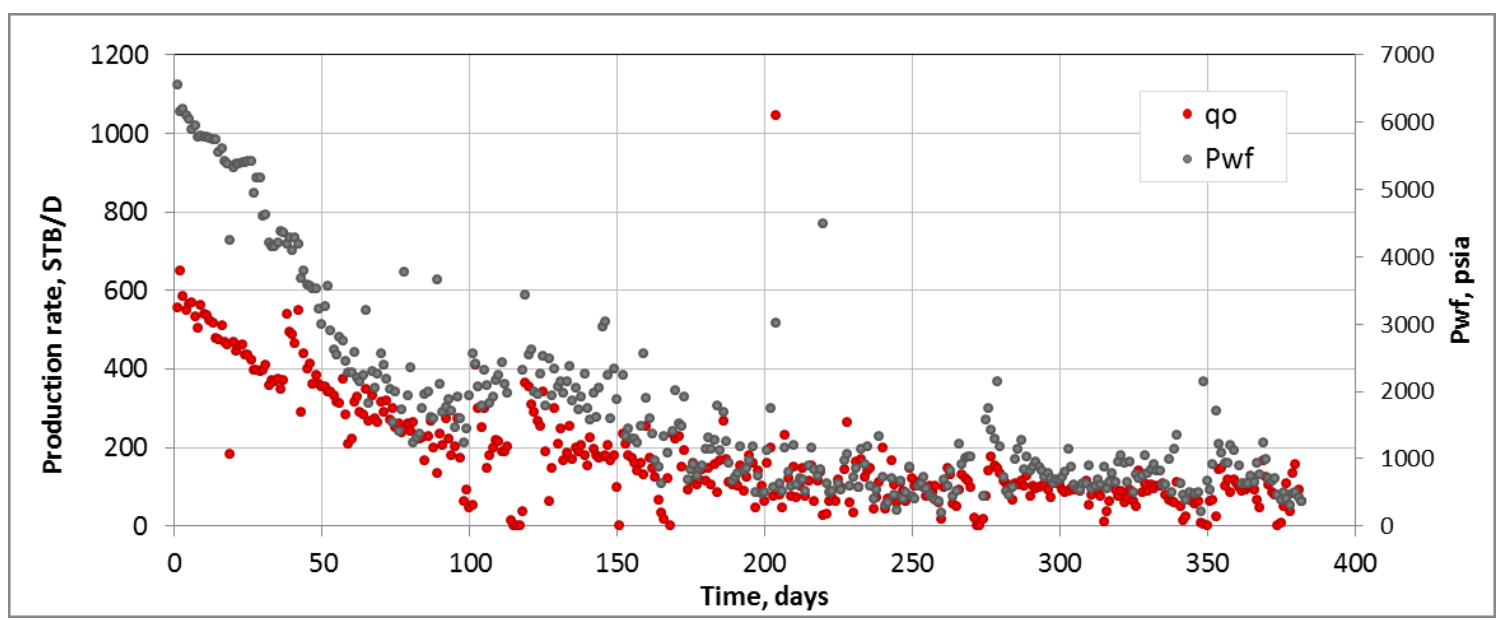

Fig. 44-Production data for well BK-2

\subsubsection{Flow Regimes Diagnosis}

Flow regimes identification (FRI) has been developed following procedures described in Section 5.1.2. Table 10 summarizes these results. A green " $x$ " marks that the corresponding flow regime has been identified by that diagnostic plot. Diagnostic plots using pseudopressures don't apply to oil wells. Thus, an "NA" mark appears on the corresponding plots for those cases. The flow regimes diagnosis was focused on the identification of linear and BDF because of their direct relation with the DCA models. However, bilinear flow was also included in this analysis. 
Table 10-Summary table, including flow regime identification results.

\begin{tabular}{|c|c|c|c|c|c|c|c|c|c|c|c|c|c|c|c|c|c|c|c|c|c|c|c|c|c|c|c|c|c|c|}
\hline \multirow{3}{*}{ Play } & \multirow{3}{*}{ Well } & \multirow{3}{*}{$\begin{array}{l}\text { Fluid } \\
\text { G/O }\end{array}$} & \multicolumn{2}{|c|}{$\begin{array}{c}\text { Production } \\
\text { History }\end{array}$} & \multicolumn{3}{|c|}{$\begin{array}{l}\text { Data } \\
\text { Quality }\end{array}$} & $\begin{array}{l}\text { Liquid } \\
\text { Loading }\end{array}$ & \multicolumn{18}{|c|}{ Flow Regime Identification } & \multirow{3}{*}{$\begin{array}{c}\text { Reliability } \\
\text { on BDF } \\
\text { Results }\end{array}$} & \multirow{2}{*}{\multicolumn{3}{|c|}{$\begin{array}{l}\text { Probability of } \\
\text { Flow Regime } \\
\text { Ocurrence }\end{array}$}} \\
\hline & & & Days & Months & B & $\mathbf{R}$ & G & Turner & \multicolumn{6}{|c|}{ Bilinear } & & & & $\mathbf{N}$ & & & & & $\mathrm{BL}$ & DF & & & & & & \\
\hline & & & No. & No. & $x$ & $x$ & $x$ & PLL/FLL & 1 & 2 & 3 & 4 & 7 & 8 & 1 & 2 & 3 & 4 & 5 & 6 & 1 & 2 & 3 & 4 & 5 & 6 & & BL & LN & BDF \\
\hline & $M-1$ & G & 1288 & 43 & & $x$ & & $60 \%$ & $x$ & $x$ & $x$ & $x$ & $x$ & $x$ & $\mathrm{x}$ & $x$ & $x$ & $x$ & $\mathrm{x}$ & $\mathrm{x}$ & $\mathrm{x}$ & $\mathrm{x}$ & $x$ & $x$ & $x$ & $\mathrm{X}$ & $60 \%$ & $100 \%$ & $100 \%$ & $60 \%$ \\
\hline & $M-2$ & G & 1187 & 40 & & $x$ & & $20 \%$ & $x$ & $x$ & $x$ & $\mathrm{x}$ & $x$ & $x$ & $x$ & $x$ & $x$ & $x$ & $x$ & $x$ & $\mathrm{X}$ & $x$ & $x$ & $x$ & $x$ & $\mathrm{x}$ & $60 \%$ & $100 \%$ & $100 \%$ & $60 \%$ \\
\hline & $M-3$ & G & 1188 & 40 & & $x$ & & $0 \%$ & $x$ & $x$ & & & & & $\mathrm{x}$ & $\mathrm{x}$ & $\mathrm{x}$ & $\mathrm{x}$ & $\mathrm{x}$ & $\mathrm{x}$ & & $x$ & $x$ & $x$ & $x$ & & $40 \%$ & $0 \%$ & $100 \%$ & $34 \%$ \\
\hline & $M-4$ & G & 1285 & 43 & & $x$ & & $100 \%$ & $x$ & $x$ & $x$ & $x$ & $x$ & & $x$ & $x$ & $x$ & $x$ & $x$ & $x$ & & $x$ & $x$ & $x$ & $x$ & $x$ & $60 \%$ & $85 \%$ & $100 \%$ & $60 \%$ \\
\hline & $M-5$ & G & 1234 & 41 & & $x$ & & $0 \%$ & $x$ & $x$ & & $x$ & & & $\mathrm{x}$ & $x$ & $\mathrm{x}$ & $x$ & $\mathrm{x}$ & $x$ & & $x$ & $x$ & $x$ & $x$ & $\mathrm{x}$ & $40 \%$ & \begin{tabular}{|l|}
$40 \%$ \\
\end{tabular} & $100 \%$ & $40 \%$ \\
\hline & M-6 & G & 1254 & 42 & $x$ & & & $60 \%$ & $x$ & $x$ & $x$ & $x$ & $x$ & $x$ & $x$ & $\mathrm{x}$ & $x$ & $x$ & $x$ & $x$ & & $x$ & $x$ & $x$ & $x$ & $x$ & $20 \%$ & $100 \%$ & $100 \%$ & $20 \%$ \\
\hline M & $M-7$ & G & 1254 & 42 & $x$ & & & $81 \%$ & $x$ & $x$ & $x$ & & $x$ & & $x$ & $x$ & $x$ & $x$ & $x$ & $\mathrm{x}$ & & $x$ & $x$ & $x$ & & $\mathrm{x}$ & $10 \%$ & $45 \%$ & $100 \%$ & $10 \%$ \\
\hline iviarcenus & $M-8$ & G & 1226 & 41 & & $x$ & & $0 \%$ & $x$ & $x$ & $x$ & $x$ & $x$ & $x$ & $x$ & $x$ & $x$ & $x$ & $\mathrm{x}$ & $\mathrm{x}$ & & $x$ & $x$ & $x$ & & $\mathrm{x}$ & $60 \%$ & $100 \%$ & $100 \%$ & $57 \%$ \\
\hline & M-9 & G & 1135 & 38 & & $x$ & & $0 \%$ & $x$ & $x$ & $x$ & $x$ & $x$ & $x$ & $x$ & $x$ & $x$ & $x$ & $x$ & $x$ & & $x$ & & & & $x$ & $70 \%$ & $100 \%$ & $100 \%$ & $11 \%$ \\
\hline & M-10 & G & 1156 & 39 & & $x$ & & $29 \%$ & $x$ & $x$ & $x$ & $x$ & $x$ & $x$ & $x$ & $x$ & $x$ & $x$ & $x$ & $x$ & & $x$ & $x$ & $x$ & & $x$ & $60 \%$ & $100 \%$ & $100 \%$ & $57 \%$ \\
\hline & $\mathrm{M}-11$ & G & 1017 & 34 & & $x$ & & $0 \%$ & $x$ & $x$ & $x$ & & $x$ & & $\mathrm{x}$ & $x$ & $x$ & $x$ & $\mathrm{x}$ & $\mathrm{x}$ & & $x$ & & $x$ & & & $70 \%$ & \begin{tabular}{|l|}
$45 \%$ \\
\end{tabular} & $100 \%$ & $28 \%$ \\
\hline & $M-12$ & G & 1226 & 41 & & $x$ & & $29 \%$ & $x$ & $x$ & $x$ & $x$ & $x$ & $x$ & $\mathrm{x}$ & $x$ & $x$ & $x$ & $\mathrm{x}$ & $x$ & & $\mathrm{x}$ & $x$ & & & & $40 \%$ & $100 \%$ & $100 \%$ & $16 \%$ \\
\hline & M-13 & G & 1161 & 39 & & $x$ & & $20 \%$ & $x$ & $x$ & $x$ & & $x$ & & $x$ & $x$ & $x$ & $x$ & $x$ & $x$ & & $x$ & $x$ & $x$ & & $\mathrm{x}$ & $40 \%$ & $45 \%$ & $100 \%$ & $38 \%$ \\
\hline & $\mathrm{M}-14$ & G & 1016 & 34 & & $x$ & & $0 \%$ & $x$ & $x$ & $x$ & & $x$ & & $\mathrm{x}$ & $x$ & $x$ & $x$ & $\mathrm{x}$ & $x$ & & $\mathrm{x}$ & & $\mathrm{x}$ & & & $60 \%$ & \begin{tabular}{|l|}
$45 \%$ \\
\end{tabular} & $100 \%$ & $24 \%$ \\
\hline & WF-1 & G & 1304 & 44 & & & $x$ & $100 \%$ & $x$ & $x$ & $x$ & $\mathrm{x}$ & $x$ & $x$ & $x$ & $x$ & $x$ & $x$ & $\mathrm{x}$ & $\mathrm{x}$ & & $x$ & & & & & $100 \%$ & $100 \%$ & $100 \%$ & $0 \%$ \\
\hline & WF-2 & G & 793 & 27 & & & $x$ & $100 \%$ & $x$ & $x$ & $x$ & $x$ & $x$ & $x$ & $\mathrm{x}$ & $x$ & $\mathrm{x}$ & $x$ & $\mathrm{x}$ & $\mathrm{x}$ & & $x$ & & & & & $100 \%$ & $100 \%$ & $100 \%$ & $0 \%$ \\
\hline & WF-3 & G & 1138 & 38 & & & $x$ & $100 \%$ & $x$ & $x$ & $x$ & & $x$ & $x$ & $\mathrm{x}$ & $x$ & $x$ & $x$ & & & & $x$ & & & & \begin{tabular}{|l|l|}
$x$ \\
\end{tabular} & $100 \%$ & \begin{tabular}{|l|}
$60 \%$ \\
\end{tabular} & \begin{tabular}{|l|}
$80 \%$ \\
\end{tabular} & $15 \%$ \\
\hline & WF-4 & G & 1314 & 44 & & & $x$ & $100 \%$ & $x$ & $x$ & $x$ & $x$ & $x$ & $x$ & $x$ & $\mathrm{x}$ & $x$ & $x$ & $x$ & $x$ & & $x$ & & & & $x$ & $100 \%$ & $100 \%$ & $100 \%$ & $15 \%$ \\
\hline & WF-5 & G & 1315 & 44 & & & $x$ & $100 \%$ & $x$ & $x$ & & & $x$ & $x$ & $x$ & $x$ & $x$ & $\mathrm{X}$ & $\mathrm{x}$ & $\mathrm{x}$ & & $\mathrm{x}$ & & & & & $100 \%$ & $20 \%$ & $100 \%$ & $0 \%$ \\
\hline W/ & WF-6 & G & 1289 & 43 & & $x$ & & $100 \%$ & $x$ & $x$ & & & & & $\mathrm{x}$ & $x$ & $x$ & $x$ & $\mathrm{x}$ & $\mathrm{x}$ & & $x$ & & & & & $100 \%$ & $0 \%$ & $100 \%$ & $0 \%$ \\
\hline vovouiota & WF-7 & G & 570 & 19 & & & $x$ & $100 \%$ & $x$ & $x$ & $x$ & & $x$ & & $\mathrm{x}$ & $x$ & $\mathrm{x}$ & $x$ & $\mathrm{x}$ & $x$ & & & & & $x$ & $x$ & $100 \%$ & \begin{tabular}{|l|}
$45 \%$ \\
\end{tabular} & $100 \%$ & $20 \%$ \\
\hline & WF-8 & G & 565 & 19 & & & $x$ & $100 \%$ & $x$ & $x$ & & & & & $x$ & $x$ & $x$ & $x$ & $x$ & $x$ & & & & & & & $100 \%$ & $0 \%$ & $100 \%$ & $0 \%$ \\
\hline & WF-9 & G & 826 & 28 & & & $x$ & $100 \%$ & $x$ & $x$ & $x$ & $x$ & $x$ & $x$ & $\mathrm{x}$ & $x$ & $x$ & $x$ & $\mathrm{x}$ & $\mathrm{x}$ & & $\mathrm{x}$ & & & & & $100 \%$ & $100 \%$ & $100 \%$ & $0 \%$ \\
\hline & WF-10 & G & 1112 & 37 & & & $x$ & $100 \%$ & $x$ & $x$ & $x$ & & $x$ & $x$ & $\mathrm{x}$ & $x$ & $x$ & $x$ & $\mathrm{x}$ & $x$ & & & & & & & $100 \%$ & \begin{tabular}{|l|}
$60 \%$ \\
\end{tabular} & $100 \%$ & $0 \%$ \\
\hline & WF-11 & G & 1265 & 42 & & & $x$ & $100 \%$ & & $x$ & $x$ & & $x$ & $x$ & $x$ & $x$ & $x$ & $x$ & $x$ & $x$ & & $x$ & & & $x$ & $x$ & $100 \%$ & $60 \%$ & $100 \%$ & $20 \%$ \\
\hline & WF-12 & $\mathrm{G}$ & 1048 & 35 & & $x$ & & $100 \%$ & $x$ & $x$ & & & $x$ & $x$ & & $x$ & $x$ & $x$ & $\mathrm{x}$ & $\mathrm{x}$ & $x$ & $x$ & & $x$ & & $x$ & $70 \%$ & \begin{tabular}{|l|}
$20 \%$ \\
\end{tabular} & $100 \%$ & $39 \%$ \\
\hline & EF-1 & 0 & 437 & 17 & $x$ & & & -- & $x$ & $x$ & & NA & & $\mathrm{NA}$ & & & $x$ & NA & $\mathrm{x}$ & NA & & $x$ & $x$ & NA & $x$ & NA & $40 \%$ & \begin{tabular}{|l|}
$20 \%$ \\
\end{tabular} & \begin{tabular}{|l|}
$80 \%$ \\
\end{tabular} & $40 \%$ \\
\hline & EF-2 & 0 & 421 & 16 & $x$ & & & -- & & & $x$ & $\mathrm{NA}$ & $x$ & $\mathrm{NA}$ & & & $x$ & $\mathrm{NA}$ & $\mathrm{x}$ & $\mathrm{NA}$ & & $x$ & $x$ & $N A$ & $x$ & $\mathrm{NA}$ & $60 \%$ & $80 \%$ & $80 \%$ & $60 \%$ \\
\hline & $E F-3$ & 0 & 414 & 15 & & & $x$ & -- & & & $x$ & NA & $x$ & NA & & $x$ & $x$ & NA & $x$ & NA & $\mathrm{x}$ & $x$ & $x$ & NA & $x$ & NA & $70 \%$ & \begin{tabular}{|l|}
$80 \%$ \\
\end{tabular} & $100 \%$ & $70 \%$ \\
\hline & EF-4 & G & 310 & 11 & & $x$ & & $100 \%$ & $x$ & & $x$ & $x$ & $x$ & $x$ & & & $x$ & $x$ & $\mathrm{x}$ & $x$ & & $\mathrm{x}$ & $x$ & $x$ & $x$ & $x$ & $60 \%$ & $100 \%$ & $100 \%$ & $60 \%$ \\
\hline & EF-5 & G & 675 & 24 & $x$ & & & $100 \%$ & $x$ & $x$ & & $x$ & & $x$ & $x$ & $x$ & $x$ & $x$ & $x$ & $x$ & & $x$ & $x$ & $x$ & $x$ & $x$ & $40 \%$ & $55 \%$ & $100 \%$ & $40 \%$ \\
\hline & EF-6 & 0 & 565 & 21 & & $x$ & & -- & & & $x$ & NA & $x$ & $\mathrm{NA}$ & $\mathrm{x}$ & & $\mathrm{x}$ & NA & $\mathrm{x}$ & NA & $\mathrm{x}$ & $x$ & $x$ & NA & $x$ & NA & $70 \%$ & \begin{tabular}{|l|}
$80 \%$ \\
\end{tabular} & \begin{tabular}{|l|}
$80 \%$ \\
\end{tabular} & $70 \%$ \\
\hline & EF-7 & 0 & 420 & 16 & $x$ & & & -- & & $x$ & $x$ & NA & $x$ & NA & $\mathrm{x}$ & $x$ & & NA & $\mathrm{x}$ & NA & $\mathrm{x}$ & $x$ & $x$ & NA & $x$ & NA & $40 \%$ & $100 \%$ & \begin{tabular}{|l|}
$40 \%$ \\
\end{tabular} & $40 \%$ \\
\hline & EF-8 & 0 & 415 & 16 & & $x$ & & -- & $x$ & $x$ & & NA & $x$ & NA & $x$ & $\mathrm{x}$ & $\mathrm{x}$ & NA & $x$ & $\mathrm{NA}$ & $\mathrm{x}$ & $x$ & $x$ & NA & $x$ & NA & $70 \%$ & $40 \%$ & $100 \%$ & $70 \%$ \\
\hline Eagie rota & EF-9 & 0 & 259 & 10 & $x$ & & & -- & $x$ & $x$ & $x$ & NA & $x$ & NA & $\mathrm{x}$ & $\mathrm{x}$ & $\mathrm{x}$ & NA & $x$ & $\mathrm{NA}$ & & $x$ & $x$ & NA & $x$ & NA & $20 \%$ & $100 \%$ & $100 \%$ & $20 \%$ \\
\hline & EF-10 & 0 & 241 & 9 & $x$ & & & -- & & $x$ & & NA & $x$ & NA & $\mathrm{x}$ & $x$ & $x$ & NA & $x$ & NA & $\mathrm{x}$ & & $x$ & NA & $x$ & NA & $50 \%$ & \begin{tabular}{|l|}
$40 \%$ \\
\end{tabular} & $100 \%$ & $40 \%$ \\
\hline & EF-30 & 0 & 247 & 9 & & & $x$ & -- & $x$ & $x$ & $x$ & $\mathrm{NA}$ & $\mathrm{x}$ & $\mathrm{NA}$ & $\mathrm{x}$ & $\mathrm{x}$ & $x$ & NA & $\mathrm{x}$ & $\mathrm{NA}$ & $x$ & & $x$ & $N A$ & $x$ & $\mathrm{NA}$ & $100 \%$ & $100 \%$ & $100 \%$ & $80 \%$ \\
\hline & EF-31 & G & 467 & 16 & $x$ & & & $80 \%$ & $x$ & $x$ & $x$ & & $x$ & $x$ & & $x$ & $\mathrm{x}$ & $x$ & $\mathrm{x}$ & $\mathrm{x}$ & & & $x$ & $x$ & & & $70 \%$ & \begin{tabular}{|l|}
$60 \%$ \\
\end{tabular} & $100 \%$ & $56 \%$ \\
\hline & EF-40 & G & 609 & 21 & & & $x$ & $0 \%$ & $x$ & $x$ & $x$ & $x$ & $x$ & $x$ & $\mathrm{x}$ & $x$ & $\mathrm{x}$ & $x$ & $x$ & $x$ & & & & & $x$ & $x$ & $100 \%$ & $100 \%$ & $100 \%$ & $20 \%$ \\
\hline & EF-41 & 0 & 371 & 13 & $x$ & & & -- & $x$ & $x$ & & NA & $x$ & NA & & & $\mathrm{x}$ & NA & $x$ & $\mathrm{NA}$ & $\mathrm{x}$ & $x$ & $x$ & $\mathrm{NA}$ & $x$ & NA & $60 \%$ & $40 \%$ & $80 \%$ & $60 \%$ \\
\hline & EF-42 & 0 & 556 & 19 & & & $x$ & -- & $x$ & $x$ & $x$ & NA & $x$ & NA & $\mathrm{x}$ & $x$ & $x$ & NA & $\mathrm{x}$ & NA & $\mathrm{x}$ & $x$ & $x$ & NA & $x$ & NA & $80 \%$ & $100 \%$ & $100 \%$ & $80 \%$ \\
\hline & EF-43 & 0 & 397 & 14 & & $x$ & & -- & $x$ & $x$ & $x$ & $\mathrm{NA}$ & $x$ & $\mathrm{NA}$ & $\mathrm{x}$ & $x$ & $x$ & NA & $\mathrm{x}$ & NA & $\mathrm{X}$ & $\mathrm{x}$ & $x$ & NA & $x$ & $\mathrm{NA}$ & $80 \%$ & $100 \%$ & $100 \%$ & $80 \%$ \\
\hline & BK-1 & 0 & 382 & 14 & & $x$ & & -- & $x$ & $x$ & $x$ & NA & $x$ & $\mathrm{NA}$ & $\mathrm{x}$ & $x$ & $x$ & NA & $x$ & NA & $x$ & $x$ & $x$ & NA & $x$ & NA & $80 \%$ & $100 \%$ & $100 \%$ & $80 \%$ \\
\hline & BK-2 & 0 & 382 & 14 & & & $x$ & -- & $x$ & $x$ & $x$ & NA & $x$ & NA & $\mathrm{x}$ & $x$ & $x$ & NA & $x$ & NA & $\mathrm{x}$ & $x$ & $x$ & NA & $x$ & NA & $80 \%$ & $100 \%$ & $100 \%$ & $80 \%$ \\
\hline Bakken & BK-3 & 0 & 291 & 11 & & $x$ & & -- & $x$ & $x$ & $x$ & NA & $x$ & $\mathrm{NA}$ & $\mathrm{x}$ & $\mathrm{x}$ & $\mathrm{x}$ & NA & $x$ & $\mathrm{NA}$ & $\mathrm{x}$ & $x$ & $x$ & NA & $x$ & NA & $80 \%$ & $100 \%$ & $100 \%$ & $80 \%$ \\
\hline & BK-4 & G & 531 & 18 & $x$ & & & $100 \%$ & $x$ & $x$ & $x$ & $x$ & $x$ & $x$ & $\mathrm{x}$ & $x$ & $\mathrm{x}$ & $\mathrm{x}$ & $\mathrm{x}$ & $x$ & & & $x$ & $x$ & $x$ & $x$ & $60 \%$ & $100 \%$ & $100 \%$ & $60 \%$ \\
\hline & BK-20 & 0 & 854 & 29 & & $x$ & & -- & & & & NA & $x$ & NA & $x$ & $x$ & $x$ & NA & $\mathrm{x}$ & NA & & $x$ & & NA & & $\mathrm{NA}$ & $80 \%$ & $20 \%$ & $100 \%$ & $16 \%$ \\
\hline
\end{tabular}

Note that for convenience, the names of the diagnostic plots have been replaced by numbers, as presented below.

- Plot 1: $\log q-\log t$ 
- Plot 2: $\log q-\log M B T$

- Plot 3: $\log \mathrm{q} / \Delta \mathrm{P}-\log \mathrm{MBT}$

- Plot 4: $\log q / \Delta \Psi-\log T c a$

- Plot 5: $\Delta P / q-S R(T c)$

- Plot 6: $\Delta \Psi / \mathrm{q}-\mathrm{SR}(\mathrm{Tca})$

- Plot 7: $\Delta P / q-F R(T c)$

- Plot 8: $\Delta \Psi / \mathrm{q}-\mathrm{FR}(\mathrm{Tca})$

An important consideration when identifying bilinear flow regimes in unconventional reservoirs is the misleading effect generated by abnormally high water rates presented at initial production due to fractures clean-up. Thus, misleading bilinear flow signatures may appear as a consequence of the changing skin effect produced by the decreasing water rates just after stimulation jobs. In this study, most of wells presented abnormally high water rates during the first days of production. The majority of this data was discarded. However, some wells continue to exhibit a soft declining water production trend, even after a few months. As bilinear flow is not a major flow regime with a significant relation to the DCA models, further analysis was not performed.

\subsubsection{Bilinear Flow - Single Method Approach}

The main objective of this section is to determine whether misinterpretations of flow regimes occur when diagnostic plots are not applied correctly. In this study, the results from the main diagnostic plot (plot 4 for gas cases and plot 3 for oil cases) are considered to be the right solution. All the other plots are referred as alternative (secondary) FRI plots. Therefore, results provided by each alternative diagnostic plot have been compared with results generated by the main (reference) FRI plots. Two parameters have been calculated to assess the effectiveness of the complementary diagnostic plots. The first parameter corresponds to the percent error $(E)$, which expresses the percentage difference between main and alternative methods. A positive difference (percent error) means that the complementary method overestimated the number of wells exhibiting that particular flow regime. On the contrary, a negative difference (percent error) means that the complementary method is underestimating 
the number of wells exhibiting that particular flow regime. The second parameter is the matching index, which represents the accuracy of the alternative methods. A match of $100 \%$ means that both methods (main and alternative) agreed in all the cases. $0 \%$ match means that the wells that the alternative plot identified don't correspond to those wells indicated by the main FRI plot. Then the alternative plot is completely wrong, even if there is not any difference on the number of estimations. The ideal combination of parameters is $0 \%$ difference (not overestimation/underestimation) and 100\% match (accurate predictions).

In general, 16 out of 31 gas wells (52\%) and 11 out of 16 oil wells (69\%) exhibited the bilinear flow signature. Table 11 summarizes results of flow regimes analysis. Results have been separated by fluid type. Main outputs are encircled in red to be easily distinguishable from alternative methods.

Table 11-Comparison of FRI results for gas and oil wells.

\begin{tabular}{|c|c|c|c|c|c|c|c|c|c|c|c|c|c|c|c|c|}
\hline \multirow{3}{*}{$\begin{array}{l}\text { Fluid Type } \\
\text { Flow Regimes } \\
\text { Diagnostic plot }\end{array}$} & \multicolumn{8}{|c|}{ Gas (31 wells) } & \multicolumn{8}{|c|}{ Oil (16 wells) } \\
\hline & \multicolumn{4}{|c|}{ Bilinear } & \multicolumn{4}{|c|}{ BDF } & \multicolumn{4}{|c|}{ Bilinear } & \multicolumn{4}{|c|}{ BDF } \\
\hline & Wells & $\%$ & Match & $E(\%)$ & Wells & $\%$ & Match & $E(\%)$ & Wells & $\%$ & Match & $E(\%)$ & Wells & $\%$ & Match & $E(\%)$ \\
\hline $\log q-\log t$ & 29 & $94 \%$ & $100 \%$ & $81 \%$ & 3 & $10 \%$ & $19 \%$ & \begin{tabular}{|l|}
$-81 \%$ \\
\end{tabular} & 10 & $63 \%$ & $64 \%$ & $-9 \%$ & 12 & $75 \%$ & $80 \%$ & $-20 \%$ \\
\hline $\log q-\log M B T$ & 29 & $94 \%$ & $94 \%$ & $81 \%$ & 25 & $81 \%$ & $94 \%$ & $56 \%$ & 12 & $75 \%$ & $73 \%$ & $9 \%$ & 14 & $88 \%$ & $87 \%$ & $-7 \%$ \\
\hline $\log q / \Delta P-\log M B T$ & 23 & $74 \%$ & $88 \%$ & $44 \%$ & 14 & $45 \%$ & $81 \%$ & $-13 \%$ & 11 & $69 \%$ & - & - & 15 & $94 \%$ & - & - \\
\hline $\log q / \Delta \Psi-\log T c a$ & 16 & $52 \%$ & -- & -- & 16 & $52 \%$ & -- & -- & - & - & -- & -- & - & - & -- & -- \\
\hline$\Delta P / q-S R(T C)$ & 26 & $84 \%$ & $88 \%$ & $63 \%$ & 11 & $35 \%$ & $50 \%$ & $-31 \%$ & 15 & $94 \%$ & $100 \%$ & $36 \%$ & 15 & $94 \%$ & $100 \%$ & $0 \%$ \\
\hline$\Delta \Psi / q-S R(T c a)$ & 20 & $65 \%$ & $88 \%$ & $25 \%$ & 18 & $58 \%$ & $75 \%$ & $13 \%$ & - & - & - & - & - & -- & - & - \\
\hline
\end{tabular}

For gas wells, according to the first FRI plot ( $\log q-\log t), 29$ of 31 gas wells exhibited the bilinear signature, which correspond to $94 \%$ of total number of gas wells. Although there is a complete match $(100 \%)$, this method overestimated the number of wells with bilinear flow ( $81 \%$ of difference or percent error), because the right estimation is 16 wells instead of 29 wells. Fig. 45 and Fig. 46 illustrate these results (dotted red and blue lines indicate ideal 
parameters). Note that matching indexes are good, but most of plots overestimated the number of wells with bilinear pattern. The specialized bilinear plot $[\Delta \Psi / \mathrm{q}-\mathrm{FR}(\mathrm{Tca})]$ and the third plot (Log $\mathrm{q} / \triangle \mathrm{P}$ - Log MBT) provided the lower percent error (lower overestimation).

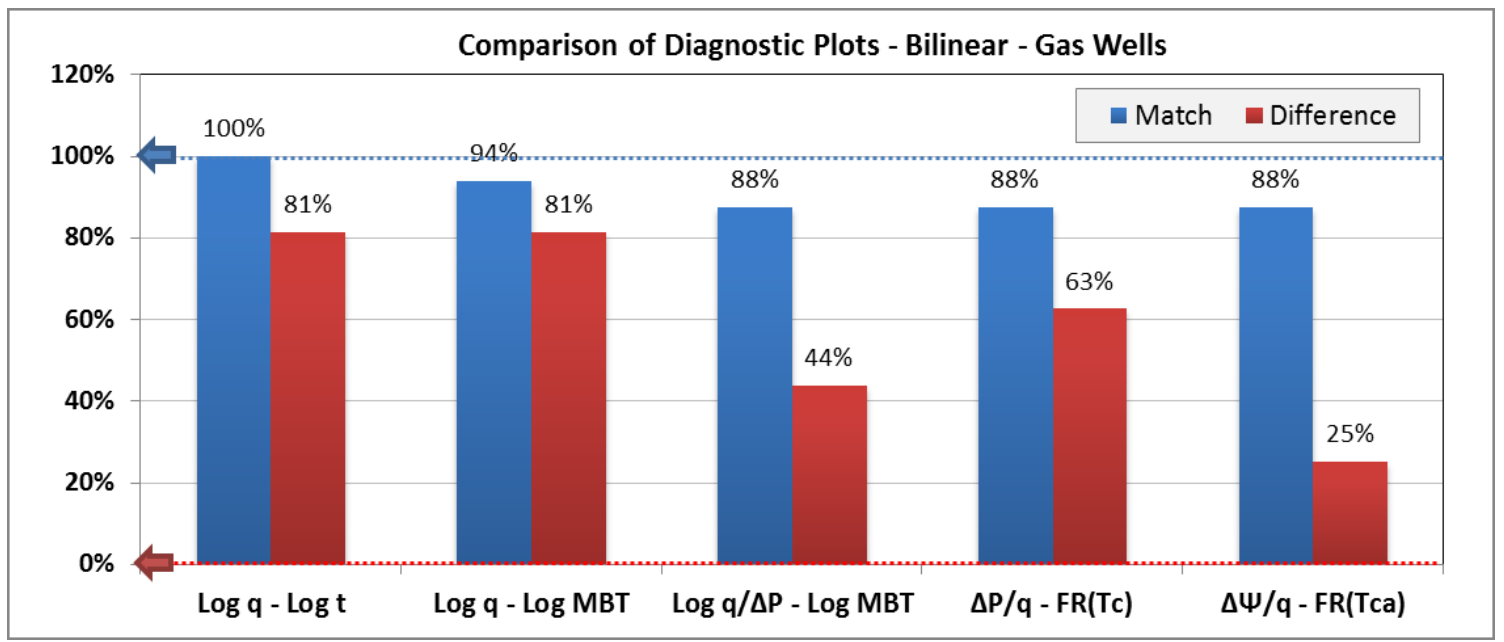

Fig. 45-Comparison of bilinear diagnostic plots for gas wells according to the single method approach.

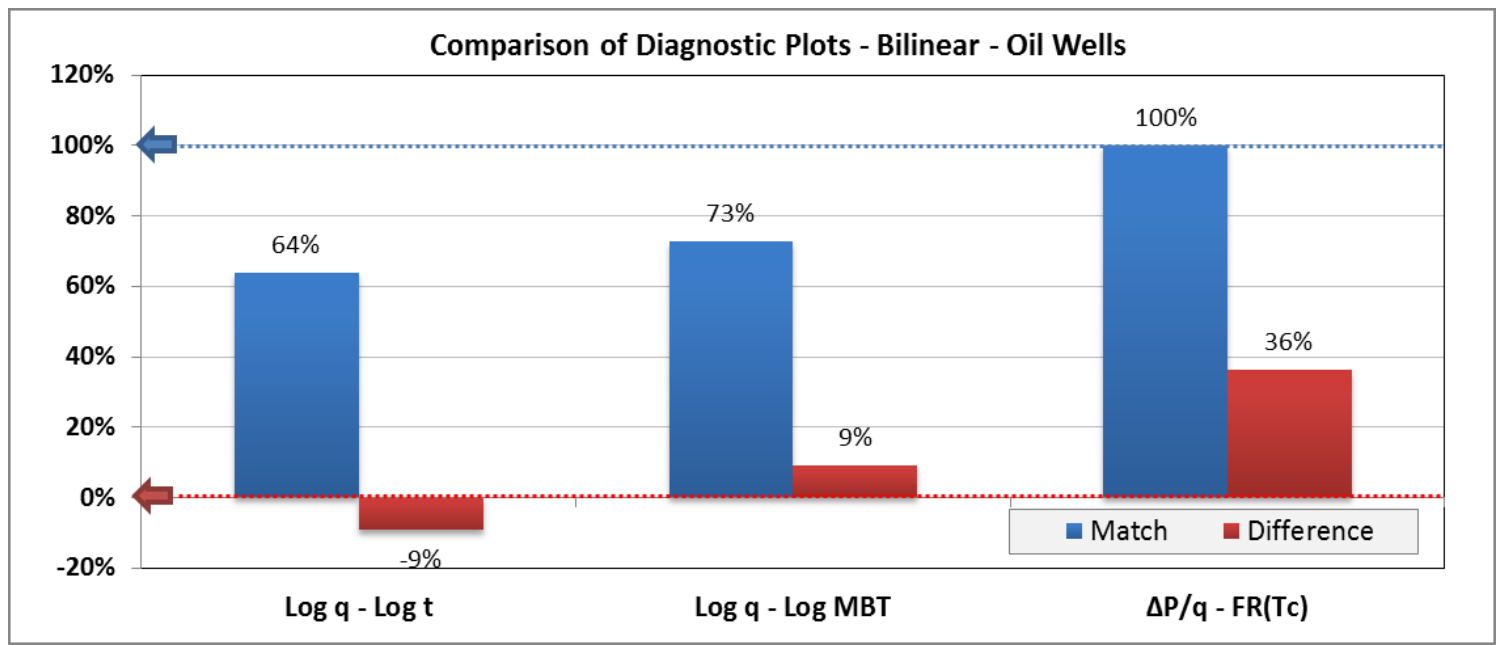

Fig. 46-Comparison of bilinear diagnostic plots for oil wells according to the single method approach. 
For oil wells, the $\Delta \mathrm{P} / \mathrm{q}-\mathrm{SR}(\mathrm{Tc})$ plot showed a perfect match (Fig. 46), although the percent error was the highest (overestimation).

\subsubsection{Bilinear Flow - Multi-Method Approach}

According to the multi-method approach, it is very likely that bilinear flow had occurred in 26 out of 47 wells (55\%). It is probable that 14 more wells had exhibited a bilinear pattern. In addition, three wells didn't show any signal of bilinear flow, while four other wells showed weak indications of bilinear flow (See Table 12). According to the single method approach, 27 wells exhibited bilinear flow signature, which is in accordance with the estimation obtained in the "very likely" category from the multi-method approach (26 wells).

Table 12-Bilinear flow diagnosis according to the multi-method approach.

\begin{tabular}{|c|r|r|r|r|r|r|r|r|r|}
\hline \multirow{2}{*}{ Play } & \multirow{2}{*}{$\begin{array}{c}\text { Total } \\
\text { Wells }\end{array}$} & \multicolumn{2}{|c|}{$\begin{array}{c}\text { No } \\
\text { Evidence }\end{array}$} & \multicolumn{2}{c|}{ Possible } & \multicolumn{2}{c|}{ Probable } & \multicolumn{2}{c|}{ Very Likely } \\
\cline { 3 - 12 } & & wells & $\%$ & wells & $\%$ & wells & $\%$ & wells & $\%$ \\
\hline Marcellus & 14 & 1 & $7 \%$ & - & $0 \%$ & 5 & $36 \%$ & 8 & $57 \%$ \\
\hline Woodford & 12 & 2 & $17 \%$ & 2 & $17 \%$ & 4 & $33 \%$ & 4 & $33 \%$ \\
\hline Eagle Ford & 16 & - & $0 \%$ & 1 & $6 \%$ & 5 & $31 \%$ & 10 & $63 \%$ \\
\hline Bakken & 5 & - & $0 \%$ & 1 & $20 \%$ & - & $0 \%$ & 4 & $80 \%$ \\
\hline Total & $\mathbf{4 7}$ & $\mathbf{3}$ & $\mathbf{6} \%$ & $\mathbf{4}$ & $\mathbf{9 \%}$ & $\mathbf{1 4}$ & $\mathbf{3 0 \%}$ & $\mathbf{2 6}$ & $\mathbf{5 5 \%}$ \\
\hline
\end{tabular}

Fig. 47 shows results for each play by separately. Weight factors used in this study are presented in Table 13. Reliability factor for bilinear flow diagnosis was ignored because most of the wells didn't present significant signals of major discontinuities/dispersions at early times, when bilinear flow is developed. 


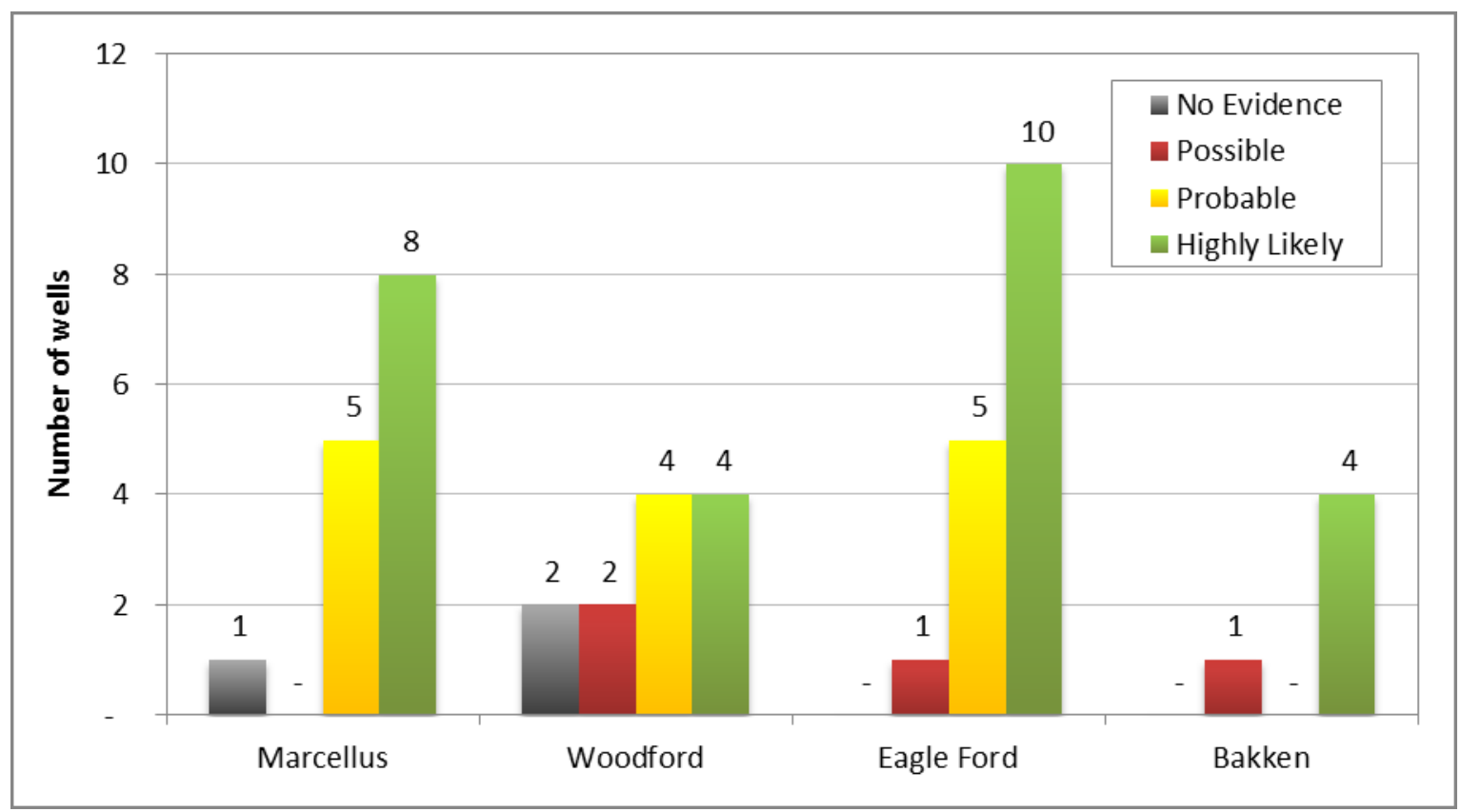

Fig. 47-Bilinear flow diagnosis according to the multi-method approach.

Table 13-Weight factors for the multi-method approach.

\begin{tabular}{|c|c|c|c|c|c|}
\hline$\#$ & Diagnostic Plot & $\begin{array}{c}\text { Bilinear } \\
\text { Gas }\end{array}$ & $\begin{array}{c}\text { Bilinear } \\
\text { Oil }\end{array}$ & $\begin{array}{l}\text { BDF } \\
\text { Gas }\end{array}$ & $\begin{array}{r}\text { BDF } \\
\text { Oil }\end{array}$ \\
\hline 1 & $\log q-\log t$ & & & & \\
\hline 2 & $\log q-\log M B T$ & & $20 \%$ & & $20 \%$ \\
\hline 3 & $\log q / \triangle P$ - Log $M B T$ & $40 \%$ & $60 \%$ & $40 \%$ & $60 \%$ \\
\hline 4 & $\log q / \Delta \Psi-\log T c a$ & $40 \%$ & & $40 \%$ & \\
\hline 5 & $\Delta P / q-S R(T c)$ & & & $5 \%$ & $20 \%$ \\
\hline 6 & $\Delta \Psi / \mathrm{q}-\mathrm{SR}(\mathrm{Tca})$ & & & $15 \%$ & \\
\hline 7 & $\Delta P / q-F R(T c)$ & $5 \%$ & $20 \%$ & & \\
\hline 8 & $\Delta \Psi / q-F R(T c a)$ & $15 \%$ & & & \\
\hline
\end{tabular}




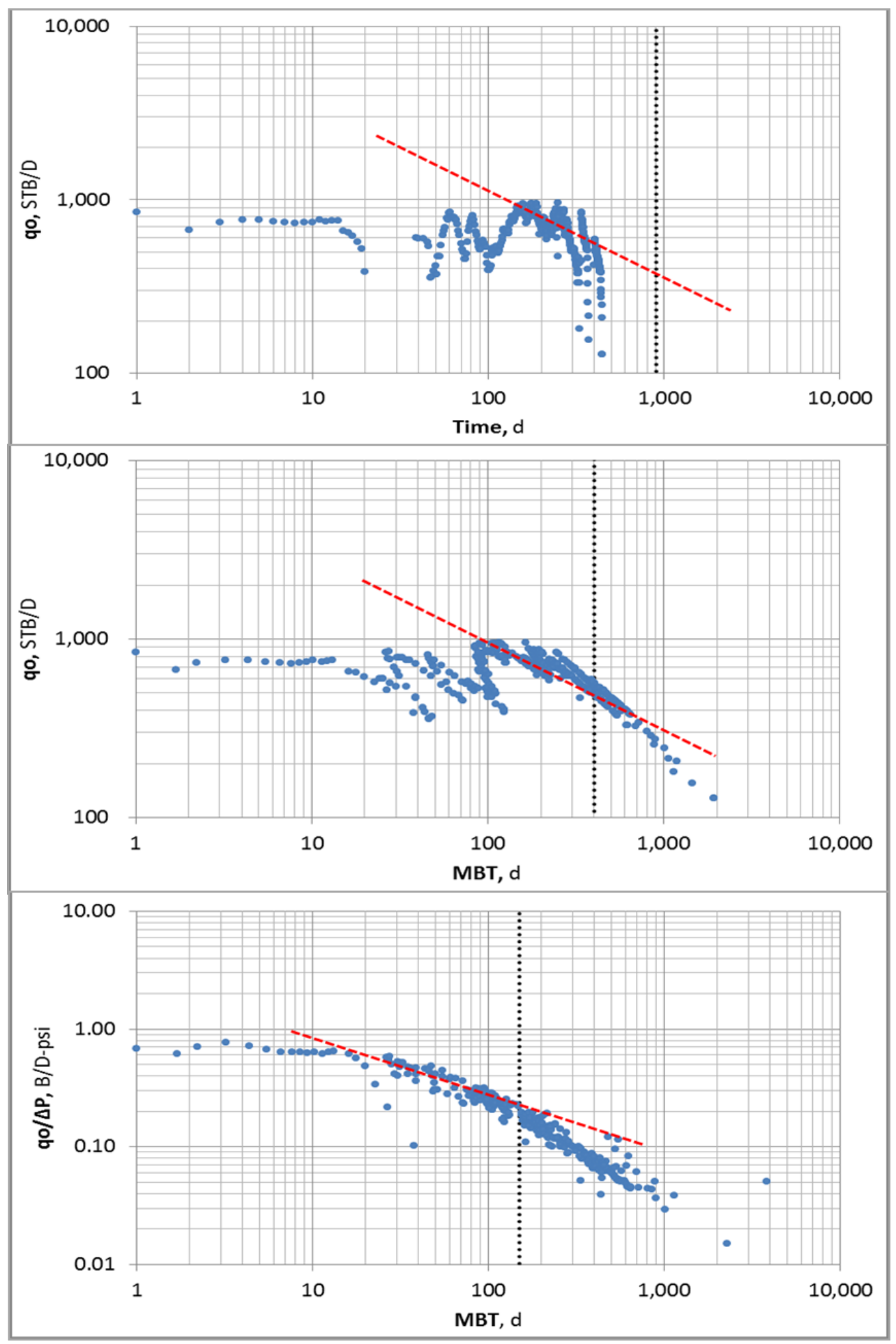

Fig. 48-Identification of linear flow regime for well EF-2. Top: Log q - Log t; middle: Log q - Log MBT; bottom: Log $q / \triangle P$ - Log MBT. Dash line represents linear flow (slope: $-1 / 2$ ). 


\subsubsection{Linear Flow}

Linear flow is the dominant flow regime in general, although a few exceptions were observed. It was readily identified by all method in majority of wells, excepting some of Eagle Ford wells, where the diagnostic plots based on production rates $(\log q-\log t$ and $\log q-\log$ MBT) did not show clearly the linear signature. But the main reason is attributable to poor quality data. In those cases, diagnostic plots based on PNR were used to improve the FRI analysis (See Fig. 48).

On the other hand, two side effects were observed in some Eagle Ford wells when using pressure-normalized rates. Seven out of 16 wells showed a weaker linear flow signature, and transition signatures get stronger in four of those wells (See Fig. 49 and Fig. 50). The effect of amplification of the transition flow was also observed in 4 more wells of Woodford play.

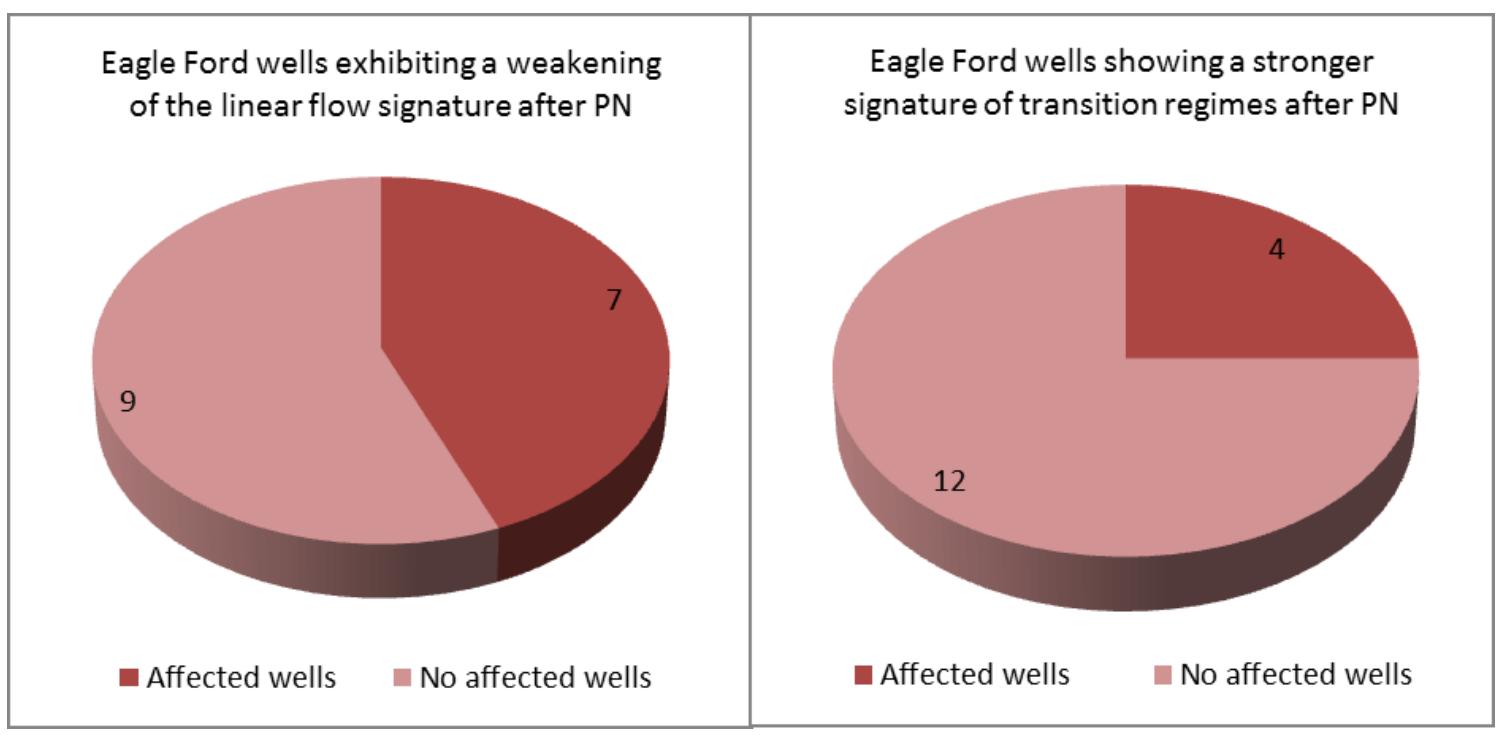

Fig. 49-Diagram showing the occurrence of side effect when using pressure-normalized rates in Eagle Ford cases. 


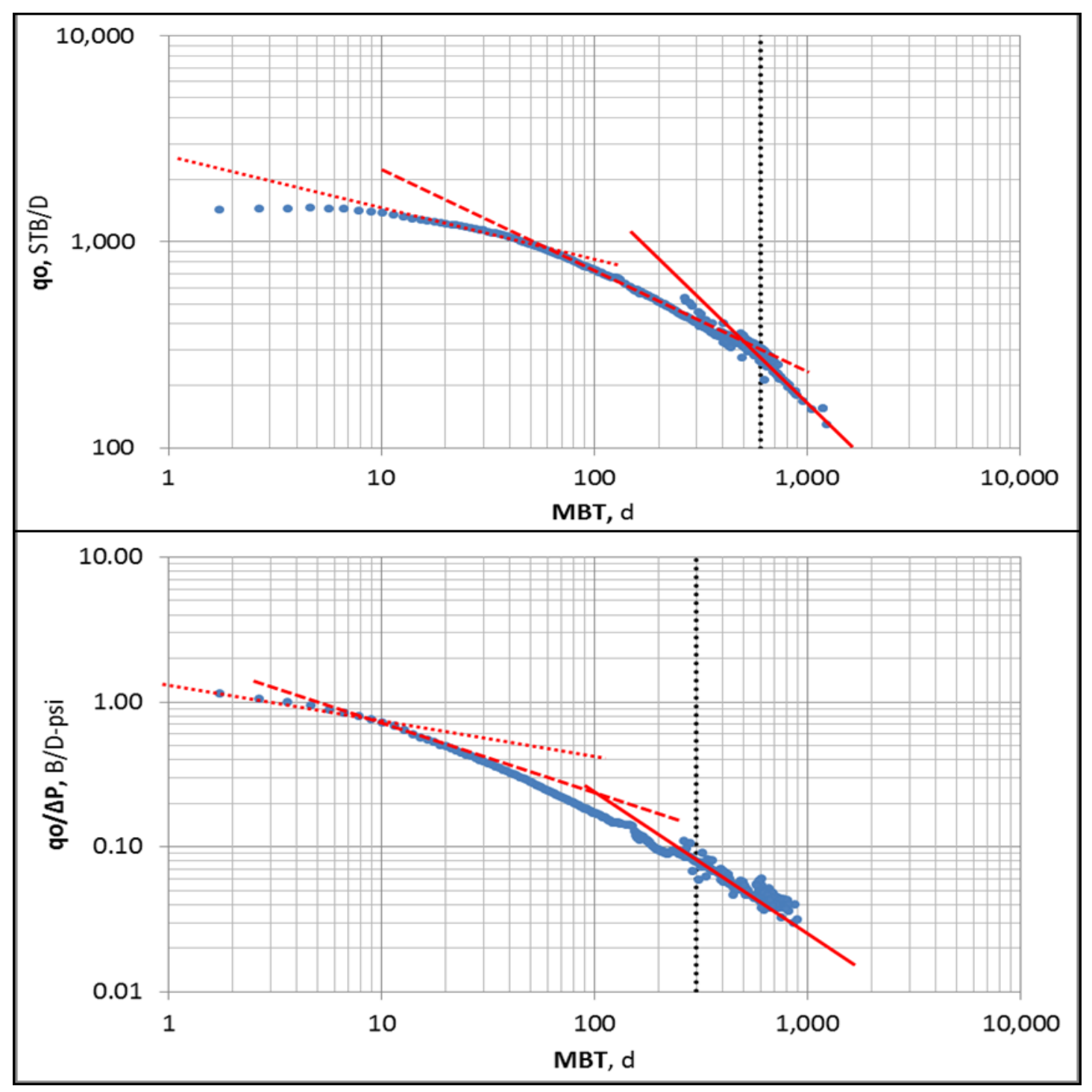

Fig. 50-Transition signature becomes dominant and linear signature shortens when pressure-normalized rates are used. Dash line: linear flow, dotted line: bilinear flow, solid line: BDF.

\subsubsection{Boundary-Dominated Flow - Single Method Approach}

In general, 16 out of 31 gas wells (52\%) and 15 out of 16 oil wells (94\%) exhibited the BDF signature. Table 11 summarizes the results of the flow regimes analysis, which have been 
separated by fluid type. Reference outputs are encircled in red to be easily distinguishable from alternative methods. Main observations are presented below:

- According to the first FRI plot ( $\log q-\log t)$, only 3 out of 31 gas wells exhibited the BDF signature, which correspond to $-81 \%$ of error-a clear indication of underestimation. Matching index is very low also (19\%), which confirms that this method provided a very poor approach.

- In the second plot $(\log q-\log M B T)$, material balance time is included and the matching index increase to $94 \%$, but the percent error is high (overestimation).

- The third method includes PNR (Log $q / \triangle P$ - Log MBT), and the difference drops to minimum level (-13\%), while match continues high (81\%).

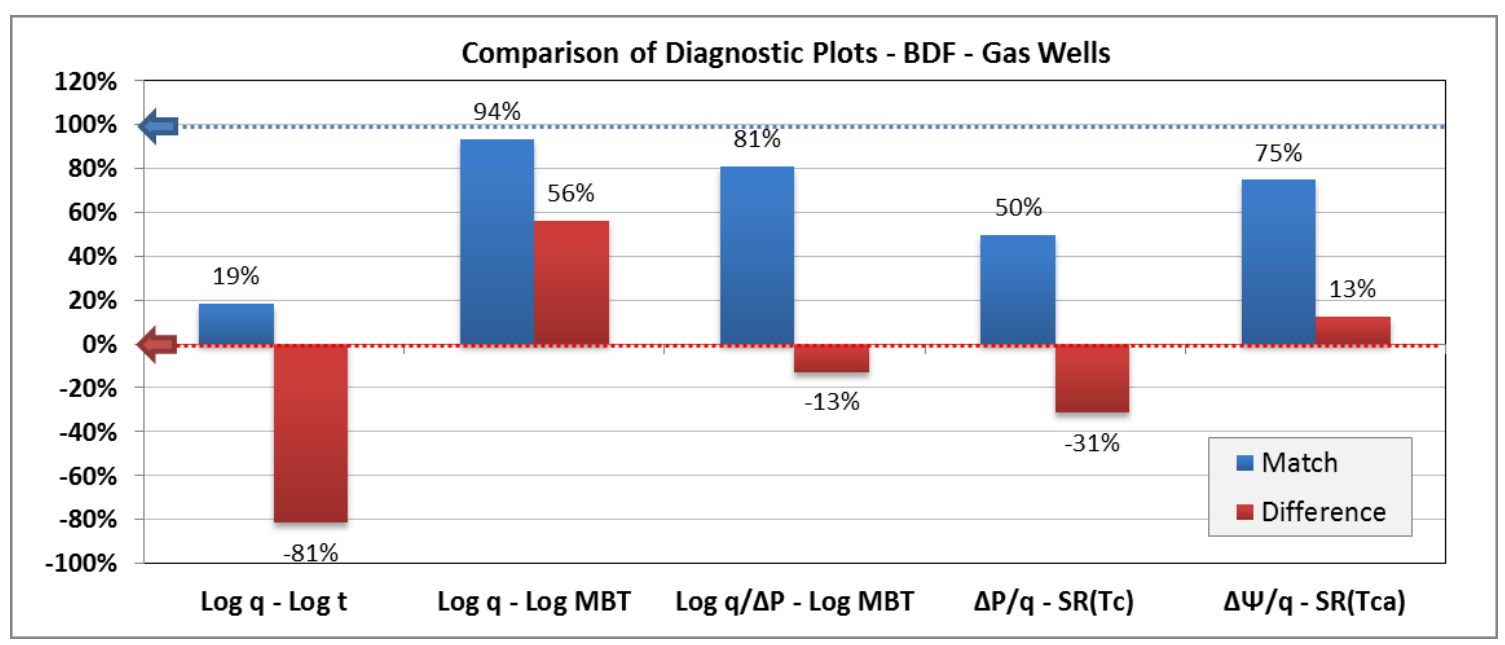

Fig. 51-Comparison of BDF diagnostic plots for gas wells according to the single method approach.

These observations indicate that more representative results are obtained as diagnostic plots are improved by including MBT and pressure-normalized rates. A similar effect happens with the specialized linear plots $[\Delta \mathrm{P} / \mathrm{q}-\mathrm{SR}(\mathrm{Tc})$ and $\Delta \Psi / \mathrm{q}-\mathrm{SR}(\mathrm{Tca})]$. Matches increase and 
differences reduce as pseudovariables are included. The same effect was observed for oil wells. Fig. 51 and Fig. 52 illustrate these results (dotted red and blue lines indicate ideal parameters).

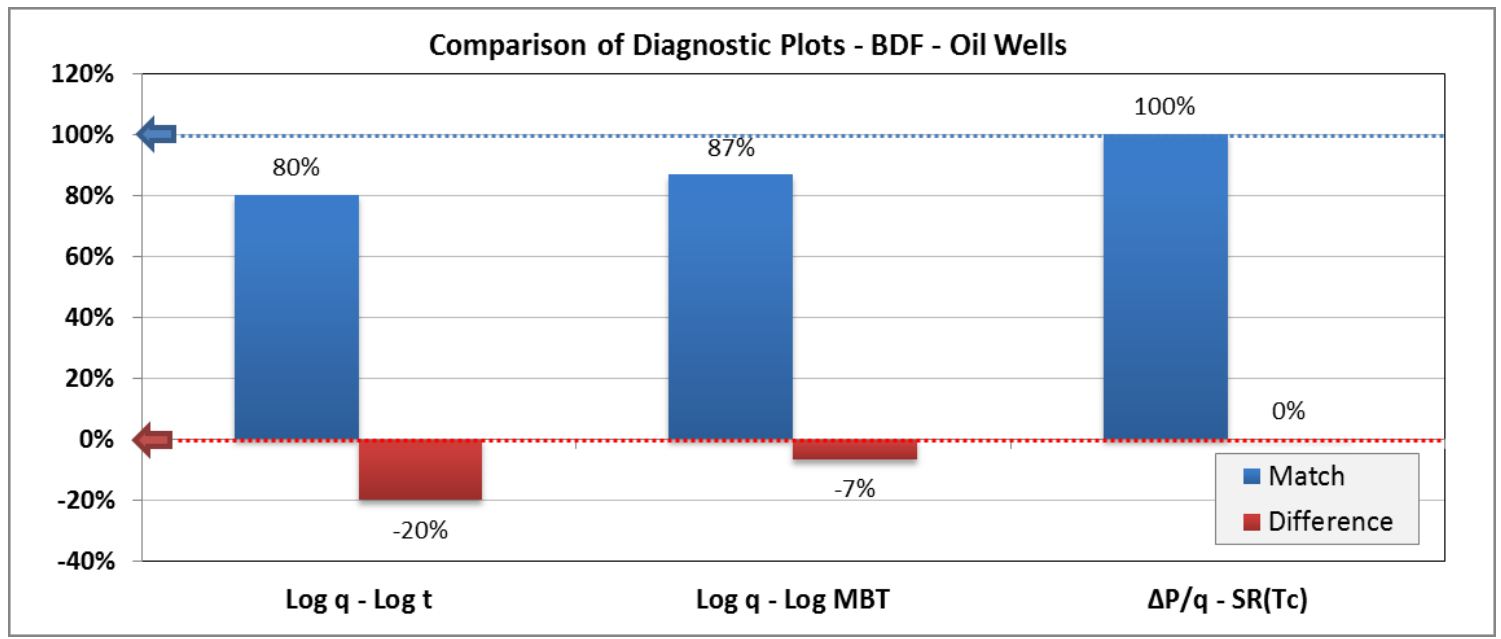

Fig. 52-Comparison of BDF diagnostic plots for oil wells according to the single method approach.

\subsubsection{Boundary-Dominated Flow - Multi-Method Approach}

According to the multi-method approach, it is very likely that BDF is exhibited in 9 out of 47 wells (55\%), and it is probable that 18 more wells are in BDF also. In addition, seven wells didn't show any BDF signal, and other 13 wells showed weak indications of a possible BDF occurrence (See Table 14). According to the single method approach, 31 wells exhibited BDF signature, which correlate with estimations obtained in the "very likely" and "probable" categories from the multi-method approach ( 27 wells). These are the wells that were considered as BDF dominated well in the hindcasting analysis. Fig. $\mathbf{5 3}$ shows results for each play by separate. Weight factors used in this study are presented in Table 13. Fig. 54 shows BDF onsets for each play. 
Table 14-BDF diagnosis by the multi-method approach

\begin{tabular}{|c|c|c|c|c|c|c|c|c|c|c|}
\hline \multirow{2}{*}{ Play } & \multirow{2}{*}{$\begin{array}{c}\text { BDF } \\
\text { Reliability } \\
\text { Factor }\end{array}$} & \multirow{2}{*}{$\begin{array}{l}\text { Total } \\
\text { Wells }\end{array}$} & \multicolumn{2}{|c|}{$\begin{array}{c}\text { No } \\
\text { Evidence }\end{array}$} & \multicolumn{2}{|c|}{ Possible } & \multicolumn{2}{|c|}{ Probable } & \multicolumn{2}{|c|}{ Very Likely } \\
\hline & & & wells & $\%$ & wells & $\%$ & wells & $\%$ & wells & $\%$ \\
\hline Marcellus & $49 \%$ & 14 & - & $0 \%$ & 6 & $43 \%$ & 8 & $57 \%$ & - & $0 \%$ \\
\hline Woodford & $98 \%$ & 12 & 7 & $58 \%$ & 4 & $33 \%$ & 1 & $8 \%$ & - & $0 \%$ \\
\hline Eagle Ford & $63 \%$ & 16 & - & $0 \%$ & 2 & $13 \%$ & 8 & $50 \%$ & 6 & $38 \%$ \\
\hline Bakken & $76 \%$ & 5 & - & $0 \%$ & 1 & $20 \%$ & 1 & $20 \%$ & 3 & $60 \%$ \\
\hline Total & $72 \%$ & 47 & 7 & $15 \%$ & 13 & $28 \%$ & 18 & $38 \%$ & 9 & $19 \%$ \\
\hline
\end{tabular}

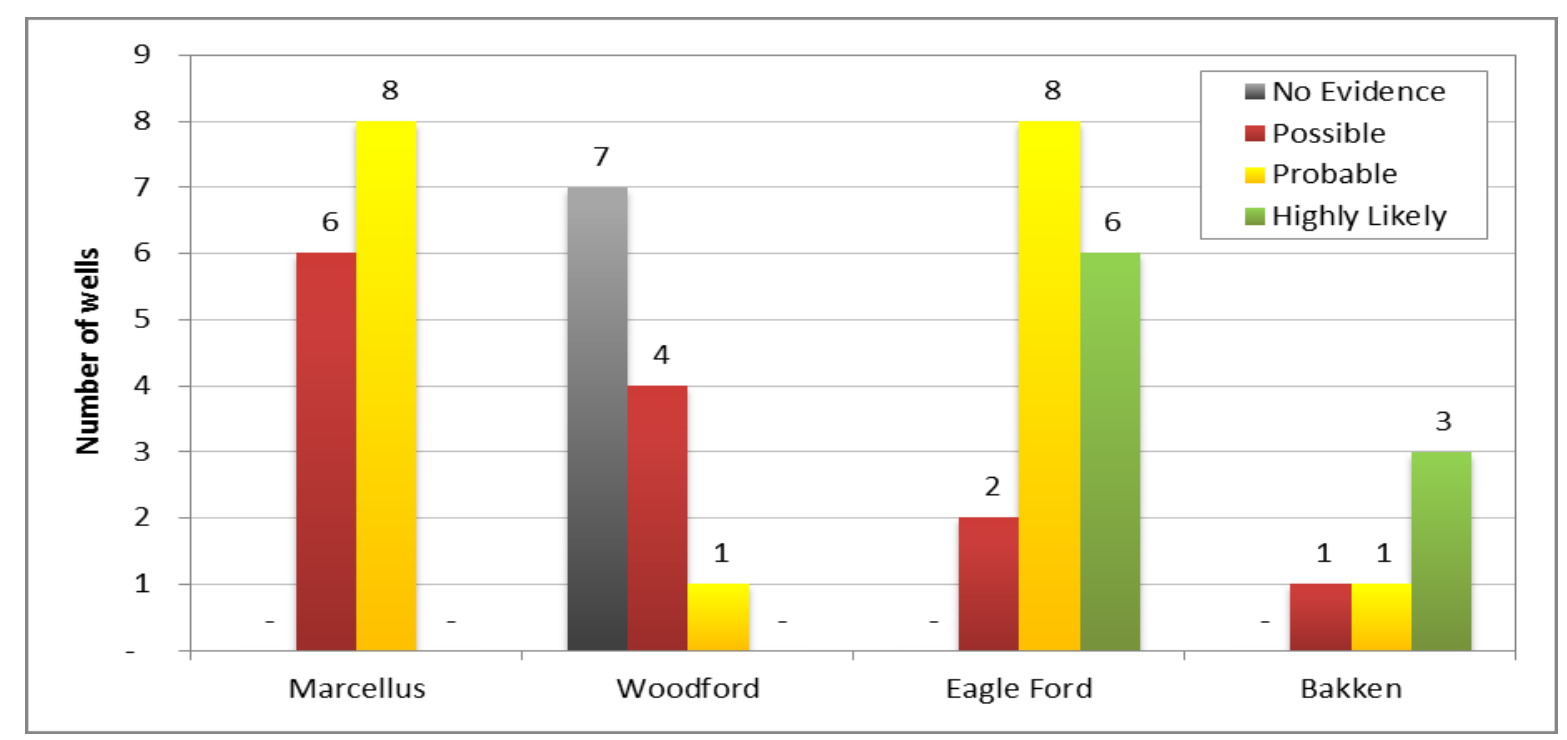

Fig. 53-BDF diagnosis according to the multi-method approach. 


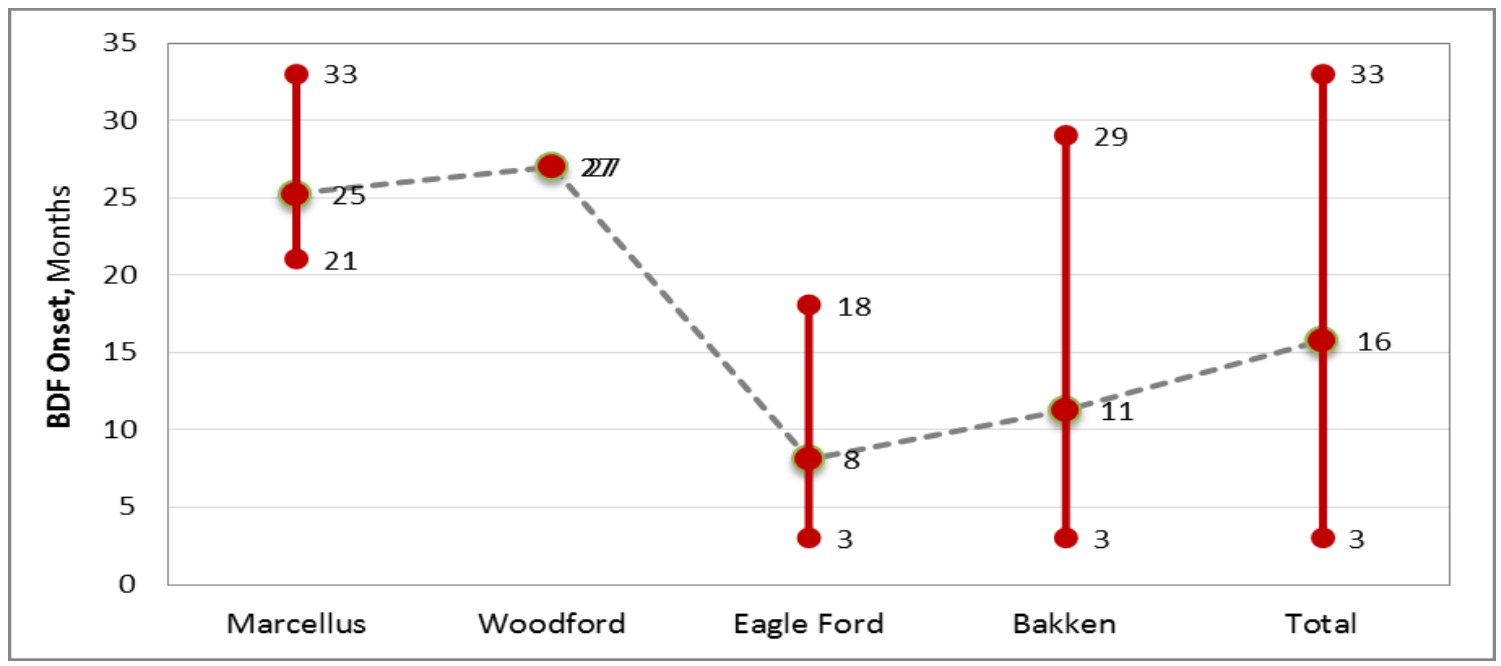

Fig. 54-Statistics of BDF onsets for each play (minimum, mean and maximum).

Using MBT in FRI diagnostic plots may lead to misinterpretation, because time functions transform the data in a way that its flow regime signatures tend to look like the flow regime for which they have been created (Clarkson, 2013b). This effect tends to increase when production data is scattered. Fig. 55 through Fig. 58 present examples of the diagnostic plots suggested in this study to identify flow regimes. Note that red lines are guide lines to ease the flow regime identification, and they don't necessarily indicate the existence of that particular flow regime. The previously mentioned effect can be easily observed in the two first plots (See plots at top of Fig. 55). Scattered data, which in the first plot appears as vertical dispersion lines, adopts an inclined straight trend with a slope equal to -1 , just like BDF. Similar behaviors were observed in most of wells with scattering problem. Unfortunately, BDF is reached at late times, just when most dispersion and discontinuities problems occur (wells are continuously intervened, liquid loading may occur in gas wells, and diverse operational changes are executed to improve production rates). This effect represents a disadvantage when using the MBT function on highly scattered data, because it increases uncertainty on estimations of the boundary-dominated flow regime. For this reason, a reliability factors for BDF flow diagnosis have been included in this methodology (See Section 5.1.2.2). In this case, reliability factors are presented in Table 10 (well by well) and Table 14 (for each play). 


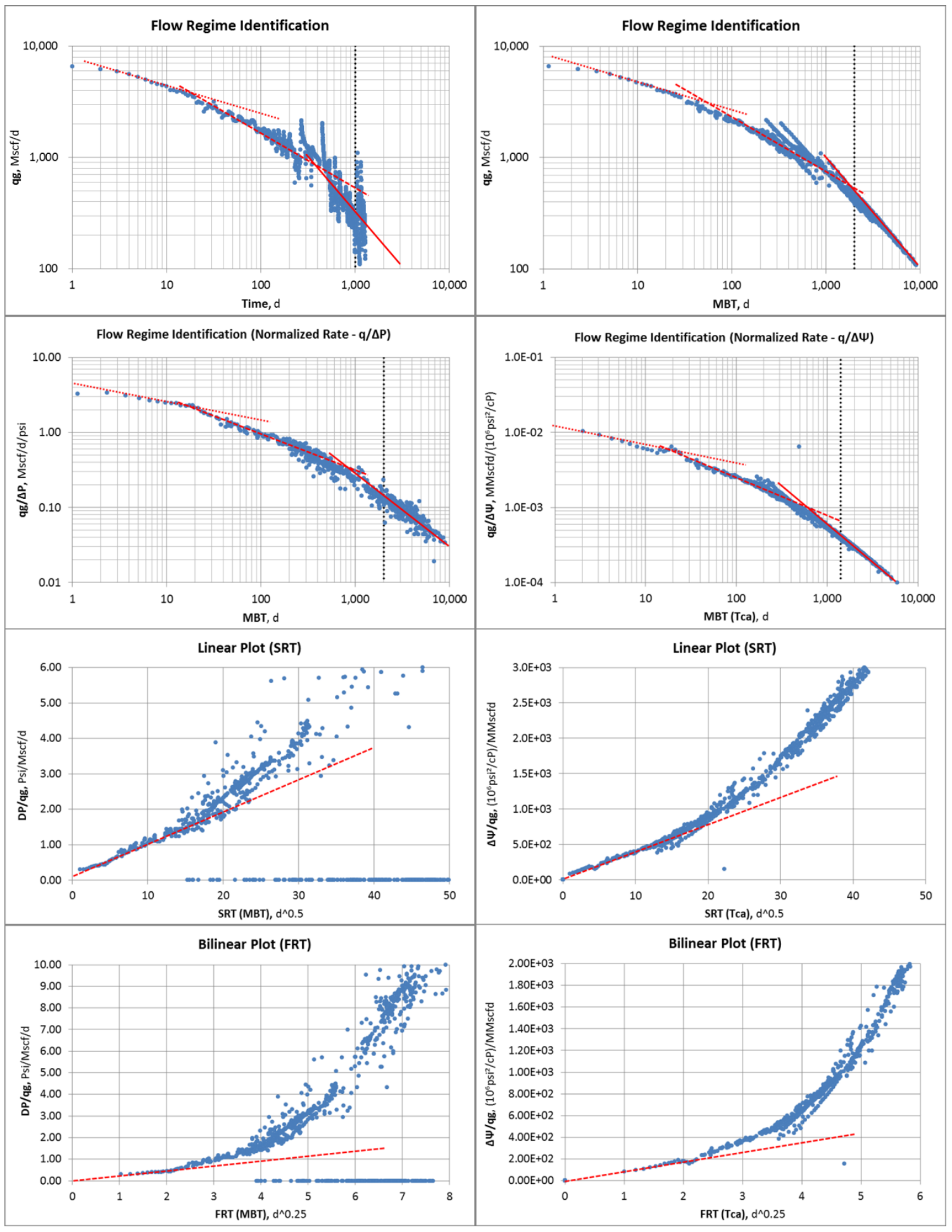

Fig. 55-FRI analysis of well M-1. Dash line: linear flow, dotted line: bilinear flow, solid line: BDF. From left to right and from top to bottom: $\log q-\log t, \log q-\log M B T, \log q / \Delta P-\log M B T, \log q / \Delta \Psi-\log T c a, \Delta P / q-S R(M B T)$, $\Delta \Psi / \mathrm{q}-\mathrm{SR}(\mathrm{Tca}), \Delta \mathrm{P} / \mathrm{q}-\mathrm{FR}(\mathrm{MBT}), \Delta \Psi / \mathrm{q}-\mathrm{FR}(\mathrm{Tca})$. 


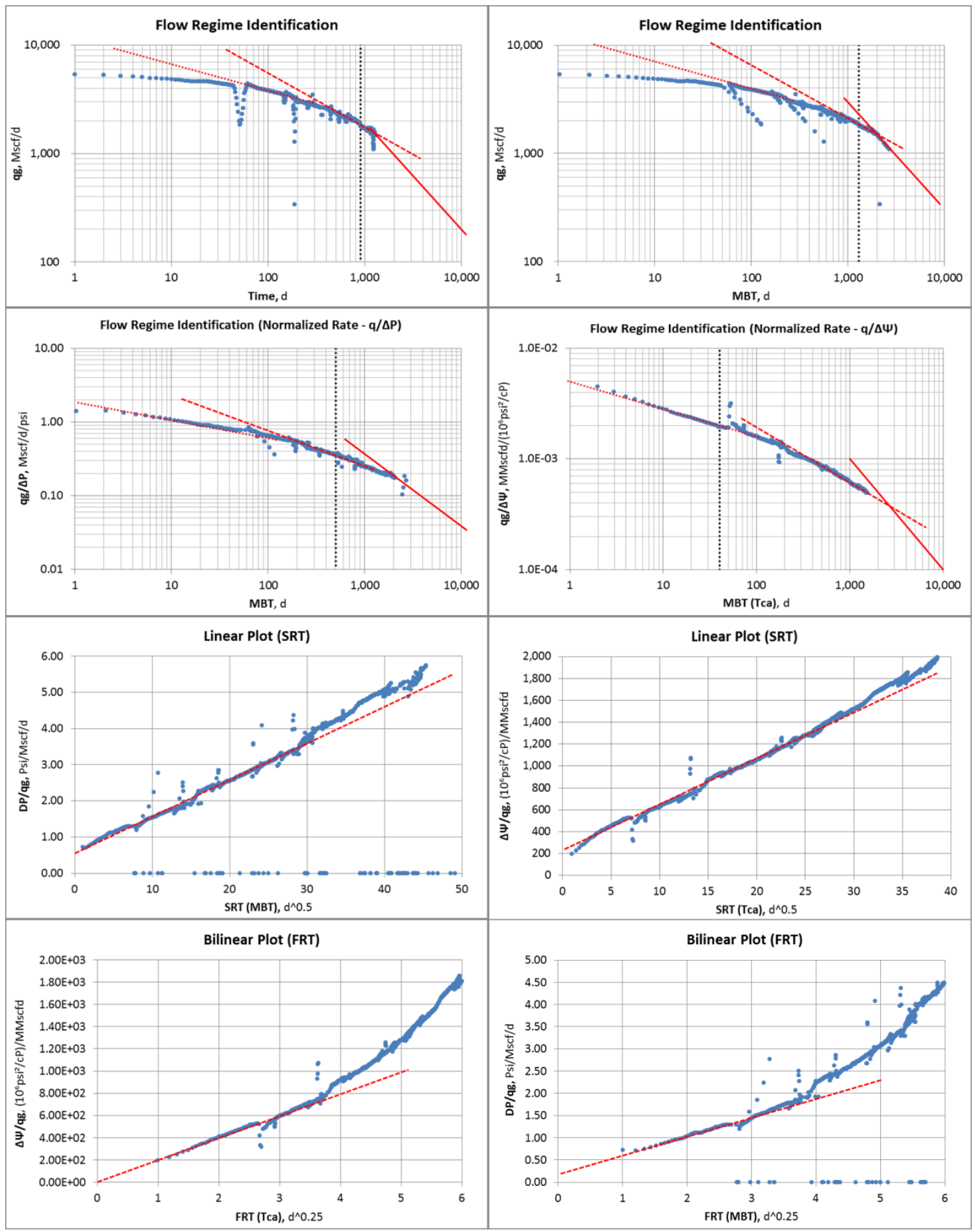

Fig. 56-FRI analysis of well WF-1. Dash line: linear flow, dotted line: bilinear flow, solid line: BDF. From left to right and from top to bottom: $\log q-\log t$, $\log q-\log M B T, \log q / \Delta P-\log M B T, \log q / \Delta \Psi-\log T c a, \Delta P / q-S R(M B T)$, $\Delta \Psi / q-S R(T c a), \Delta P / q-F R(M B T), \Delta \Psi / q-F R(T c a)$. 


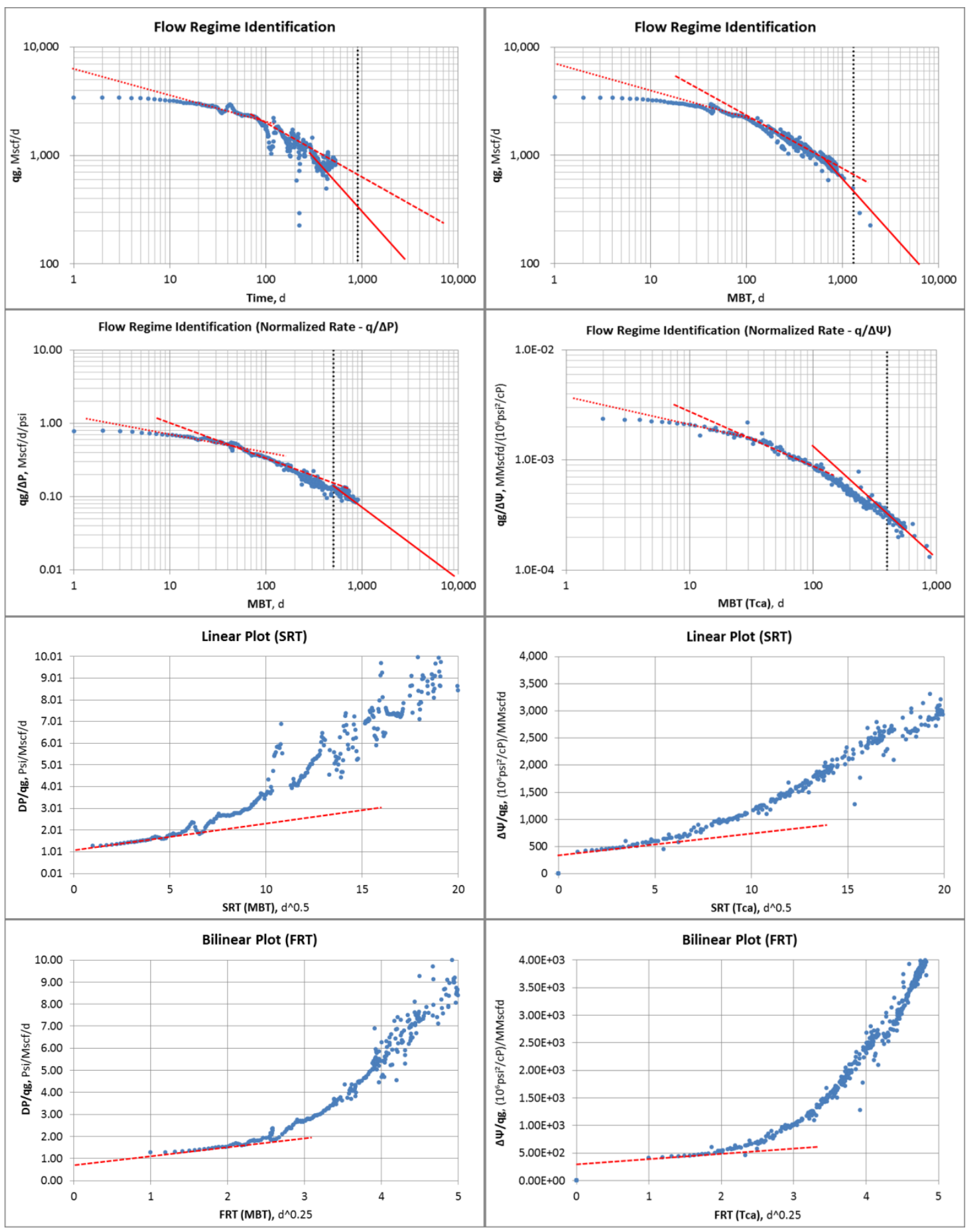

Fig. 57-FRI analysis of well BK-3. Dash line: linear flow, dotted line: bilinear flow, solid line: BDF. From left to right and from top to bottom: $\log q-\log t$, $\log q-\log M B T, \log q / \Delta P-\log M B T, \log q / \Delta \Psi-\log T c a, \Delta P / q-S R(M B T)$, $\Delta \Psi / \mathrm{q}-\mathrm{SR}(\mathrm{Tca}), \Delta \mathrm{P} / \mathrm{q}-\mathrm{FR}(\mathrm{MBT}), \Delta \Psi / \mathrm{q}-\mathrm{FR}(\mathrm{Tca})$. 


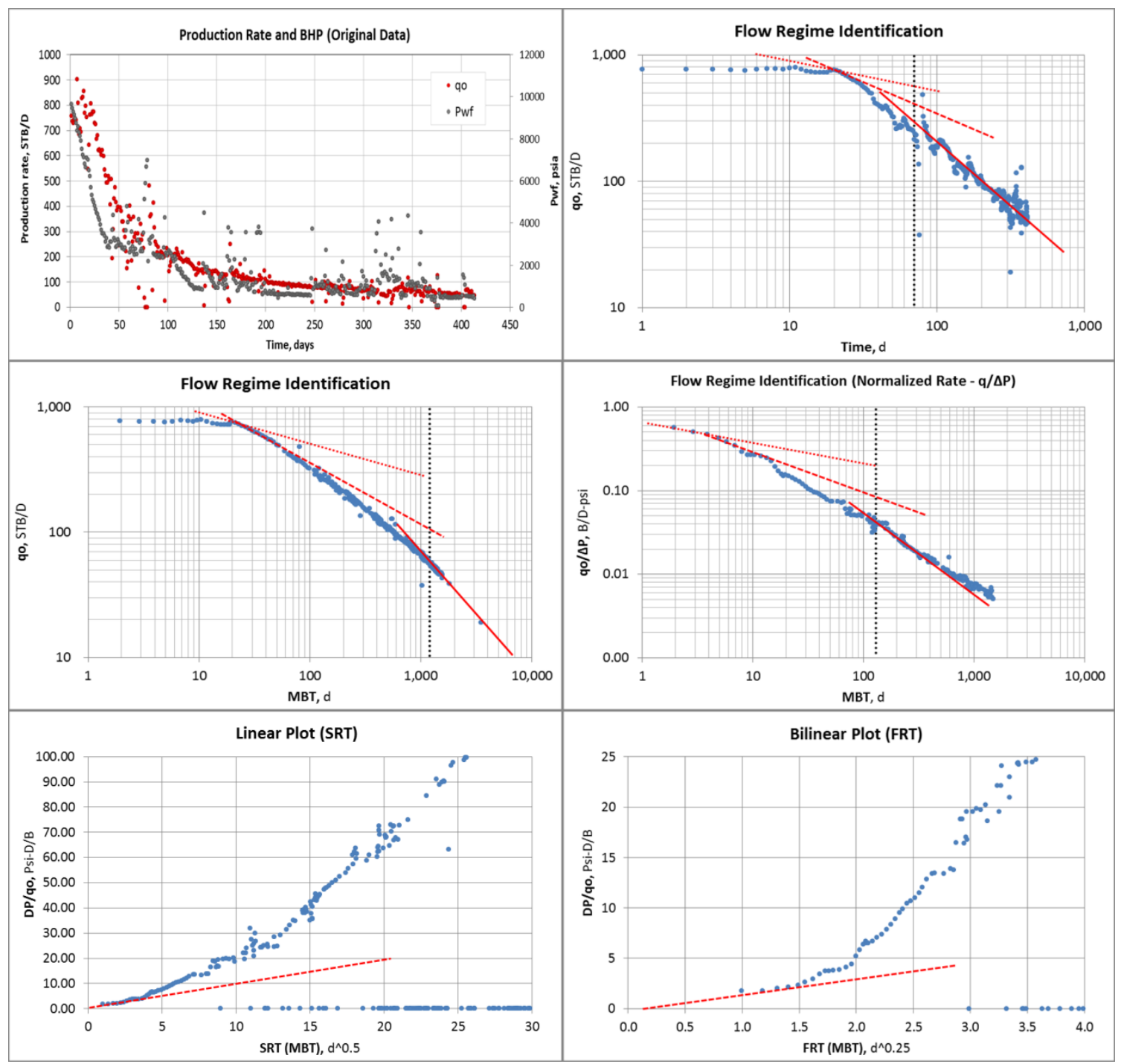

Fig. 58-FRI analysis of well EF-3. Dash line: linear flow, dotted line: bilinear flow, solid line: BDF. From left to right and from top to bottom: Production rates and flowing pressures (THP), Log $q-\log t, \log q-\log M B T, \log q / \triangle P$ - Log $M B T, \Delta P / q-S R(M B T), \Delta P / q-F R(M B T)$.

\subsubsection{Hindcasting Analysis}

The target of this hindcasting analysis is to evaluate the applicability of the pressure normalization decline curve analysis as a production forecasting technique. As discussed in 
Section 5.1.1, DCA models outputs are compared with known historic production data from a large set of real wells to determine the effectiveness and representativeness of these models.

Comparisons are based on remaining recoveries to the end of production histories and differences are expressed as percent error (See Eq. 50). Although estimations were done on a per-well basis, the comparative analysis was performed on a group basis. Thus, wells were grouped according to different features and conditions to analyze the behavior of the DCA models and the pressure normalization techniques under different scenarios. For each group, two statistic measurements of the percent error were calculated, the mean and the standard deviation. The mean represents the accuracy of the predictions and the capacity of the models to generate unbiased results (close to the real values). The standard deviation represents the precision of these estimations and the capacity of the models to consistently reproduce similar outputs. Basically, the lower the standard deviation, the more precise the method. On the other hand, the closer the mean percent error is to zero, the more accurate the method. A negative percent error means that the method tends to be conservative, underestimating the production performance. A positive percent error means that the method tends to be optimistic, overestimating the production performance.

$$
P E=\left(\frac{R R_{E s t}-R R_{\text {Real }}}{R R_{\text {Real }}}\right) * 100 \%
$$

\subsubsection{Hindcasting Scenarios}

Production data from 47 wells from 4 different plays was available. However, 42 wells were finally selected for the hindcasting analysis, and 5 wells were omitted due to poor data quality and/or short production history. 30 wells are gas producers and 12 are oil producers. Different prediction start points (hindcasting onset) have been selected for each well. Depending on the amount of production history, up to three predictions were done for a single well. Table 15 shows a summary of all the wells used in this study, including the starting times for the predictions and for the BDF regimes. 
Table 15-Wells summary table, including prediction start points and BDF onsets.

\begin{tabular}{|c|c|c|c|c|c|c|c|c|c|c|c|c|c|c|c|}
\hline \multirow{3}{*}{ Play } & \multirow{3}{*}{ Well } & \multirow{3}{*}{\begin{tabular}{|l|} 
Fluid \\
G/O \\
\end{tabular}} & \multicolumn{2}{|c|}{ Production History } & \multicolumn{3}{|c|}{ Data Quality } & \multirow{3}{*}{$\begin{array}{c}\begin{array}{c}\text { Liquid } \\
\text { Loading }\end{array} \\
\begin{array}{c}\text { Turner } \\
\text { Plot }\end{array} \\
\text { PLL/FLL }\end{array}$} & \multirow{2}{*}{\multicolumn{3}{|c|}{$\begin{array}{l}\text { Probability of Flow } \\
\text { Regime Ocurrence }\end{array}$}} & \multicolumn{3}{|c|}{ Hindcast Onsets } & \multirow{3}{*}{\begin{tabular}{|c|} 
BDF \\
Onset
\end{tabular}} \\
\hline & & & \multirow{2}{*}{$\begin{array}{c}\text { Days } \\
\text { No. }\end{array}$} & \multirow{2}{*}{$\begin{array}{c}\text { Months } \\
\text { No. }\end{array}$} & \multirow{2}{*}{$\begin{array}{l}\mathrm{B} \\
\mathrm{X}\end{array}$} & \multirow{2}{*}{$\begin{array}{l}\mathrm{R} \\
\mathrm{X}\end{array}$} & \multirow{2}{*}{\begin{tabular}{|l}
$\mathbf{G}$ \\
$\mathbf{X}$
\end{tabular}} & & & & & \multirow{2}{*}{$\begin{array}{c}\# 1 \\
\text { Months }\end{array}$} & \multirow{2}{*}{\begin{tabular}{|c|} 
\#2 \\
Months
\end{tabular}} & & \\
\hline & & & & & & & & & BL & LN & BDF & & & Months & \\
\hline & M-1 & G & 1288 & 43 & & $\mathrm{x}$ & & $60 \%$ & $100 \%$ & $100 \%$ & $60 \%$ & 7 & 17 & 23 & 24 \\
\hline & $\mathrm{M}-2$ & G & 1187 & 40 & & $\mathrm{x}$ & & $20 \%$ & $100 \%$ & $100 \%$ & $60 \%$ & 7 & 14 & 19 & 23 \\
\hline & $\mathrm{M}-3$ & G & 1188 & 40 & & $x$ & & $0 \%$ & $0 \%$ & $100 \%$ & $34 \%$ & 6 & 12 & 14 & 31 \\
\hline & $M-4$ & G & 1285 & 43 & & $\mathrm{x}$ & & $100 \%$ & $85 \%$ & $100 \%$ & $60 \%$ & 9 & 20 & 26 & 21 \\
\hline & M-5 & G & 1234 & 41 & & $\mathrm{x}$ & & $0 \%$ & $40 \%$ & $100 \%$ & $40 \%$ & 7 & 16 & 21 & 24 \\
\hline & M-6 & G & 1254 & 42 & $\mathrm{X}$ & & & $60 \%$ & $100 \%$ & $100 \%$ & $20 \%$ & 6 & 9 & 14 & -- \\
\hline Marcellus & $\mathrm{M}-7$ & $\mathrm{G}$ & 1254 & 42 & $\mathrm{X}$ & & & $81 \%$ & $45 \%$ & $100 \%$ & $10 \%$ & 7 & 10 & 16 & -- \\
\hline Triar & $M-8$ & G & 1226 & 41 & & $x$ & & $0 \%$ & $100 \%$ & $100 \%$ & $57 \%$ & 7 & 12 & 19 & 33 \\
\hline & M-9 & G & 1135 & 38 & & $x$ & & $0 \%$ & $100 \%$ & $100 \%$ & $11 \%$ & 6 & 13 & 19 & -- \\
\hline & $\mathrm{M}-10$ & G & 1156 & 39 & & $x$ & & $29 \%$ & $100 \%$ & $100 \%$ & $57 \%$ & 6 & 12 & 19 & 23 \\
\hline & $\mathrm{M}-11$ & G & 1017 & 34 & & $x$ & & $0 \%$ & $45 \%$ & $100 \%$ & $28 \%$ & 6 & 12 & 19 & -- \\
\hline & $\mathrm{M}-12$ & G & 1226 & 41 & & $x$ & & $29 \%$ & $100 \%$ & $100 \%$ & $16 \%$ & 8 & 17 & 25 & -- \\
\hline & $\mathrm{M}-13$ & G & 1161 & 39 & & $x$ & & $20 \%$ & $45 \%$ & $100 \%$ & $38 \%$ & 6 & 12 & 18 & 23 \\
\hline & $\mathrm{M}-14$ & $\mathrm{G}$ & 1016 & 34 & & $x$ & & $0 \%$ & $45 \%$ & $100 \%$ & $24 \%$ & 8 & 14 & 7 & -- \\
\hline & WF-1 & G & 1304 & 44 & & & $x$ & $100 \%$ & $100 \%$ & $100 \%$ & $0 \%$ & 6 & 16 & 22 & -- \\
\hline & WF-2 & G & 793 & 27 & & & $x$ & $100 \%$ & $100 \%$ & $100 \%$ & $0 \%$ & 6 & 14 & 17 & -- \\
\hline & WF-3 & G & 1138 & 38 & & & $x$ & $100 \%$ & $60 \%$ & $80 \%$ & $15 \%$ & 6 & 15 & 20 & -- \\
\hline & WF-4 & $\mathrm{G}$ & 1314 & 44 & & & $x$ & $100 \%$ & $100 \%$ & $100 \%$ & $15 \%$ & 6 & 15 & 22 & -- \\
\hline & WF-5 & G & 1315 & 44 & & & $X$ & $100 \%$ & $20 \%$ & $100 \%$ & $0 \%$ & 6 & 16 & 22 & -- \\
\hline Woodford & WF-6 & G & 1289 & 43 & & $x$ & & $100 \%$ & $0 \%$ & $100 \%$ & $0 \%$ & 6 & 15 & 22 & -- \\
\hline veouciora & WF-7 & G & 570 & 19 & & & $x$ & $100 \%$ & $45 \%$ & $100 \%$ & $20 \%$ & 6 & 10 & -- & -- \\
\hline & WF-8 & $\mathrm{G}$ & 565 & 19 & & & $x$ & $100 \%$ & $0 \%$ & $100 \%$ & $0 \%$ & 6 & 9 & 12 & -- \\
\hline & WF-9 & G & 826 & 28 & & & $x$ & $100 \%$ & $100 \%$ & $100 \%$ & $0 \%$ & 6 & 12 & 16 & -- \\
\hline & WF-10 & G & 1112 & 37 & & & $x$ & $100 \%$ & $60 \%$ & $100 \%$ & $0 \%$ & 6 & 12 & 18 & -- \\
\hline & WF-11 & G & 1265 & 42 & & & $x$ & $100 \%$ & $60 \%$ & $100 \%$ & $20 \%$ & 6 & 16 & 22 & -- \\
\hline & WF-12 & G & 1048 & 35 & & $x$ & & $100 \%$ & $20 \%$ & $100 \%$ & $39 \%$ & 11 & 21 & 24 & 27 \\
\hline & EF-1 & 0 & 437 & 17 & $x$ & & & -- & $20 \%$ & $80 \%$ & $40 \%$ & 5 & 10 & -- & 8 \\
\hline & $\mathrm{EF}-2$ & 0 & 421 & 16 & $X$ & & & -- & $80 \%$ & $80 \%$ & $60 \%$ & 5 & 9 & -- & 7 \\
\hline & $\mathrm{EF}-3$ & 0 & 414 & 15 & & & $x$ & -- & $80 \%$ & $100 \%$ & $70 \%$ & 5 & 8 & -- & 2 \\
\hline & $E F-4$ & G & 310 & 11 & & $x$ & & $100 \%$ & $100 \%$ & $100 \%$ & $60 \%$ & 5 & -- & -- & 2 \\
\hline & EF-5 & G & 675 & 24 & $X$ & & & $100 \%$ & $55 \%$ & $100 \%$ & $40 \%$ & 6 & 10 & 13 & 18 \\
\hline & EF-6 & 0 & 565 & 21 & & $x$ & & -- & $80 \%$ & $80 \%$ & $70 \%$ & 7 & 12 & -- & 9 \\
\hline & $\mathrm{EF}-7$ & 0 & 420 & 16 & $X$ & & & -- & $100 \%$ & $40 \%$ & $40 \%$ & -- & -- & -- & 4 \\
\hline Fagle Ford & EF-8 & 0 & 415 & 16 & & $x$ & & -- & $40 \%$ & $100 \%$ & $70 \%$ & 5 & -- & -- & 7 \\
\hline cagle rora & EF-9 & 0 & 259 & 10 & $\mathrm{X}$ & & & -- & $100 \%$ & $100 \%$ & $20 \%$ & -- & -- & -- & -- \\
\hline & EF-10 & 0 & 241 & 9 & $x$ & & & -- & $40 \%$ & $100 \%$ & $40 \%$ & -- & -- & -- & 7 \\
\hline & EF-30 & 0 & 247 & 9 & & & $x$ & -- & $100 \%$ & $100 \%$ & $80 \%$ & -- & -- & -- & 4 \\
\hline & EF-31 & $\mathrm{G}$ & 467 & 16 & $X$ & & & $80 \%$ & $60 \%$ & $100 \%$ & $56 \%$ & -- & -- & -- & 6 \\
\hline & EF-40 & G & 609 & 21 & & & $x$ & $0 \%$ & $100 \%$ & $100 \%$ & $20 \%$ & 6 & 10 & 13 & -- \\
\hline & EF-41 & 0 & 371 & 13 & $X$ & & & -- & $40 \%$ & $80 \%$ & $60 \%$ & -- & 6 & -- & 7 \\
\hline & EF-42 & 0 & 556 & 19 & & & $x$ & -- & $100 \%$ & $100 \%$ & $80 \%$ & 6 & 9 & 12 & 9 \\
\hline & EF-43 & 0 & 397 & 14 & & $x$ & & -- & $100 \%$ & $100 \%$ & $80 \%$ & 5 & -- & -- & 5 \\
\hline & BK-1 & 0 & 382 & 14 & & $x$ & & -- & $100 \%$ & $100 \%$ & $80 \%$ & -- & 5 & -- & 5 \\
\hline & BK-2 & 0 & 382 & 14 & & & $x$ & -- & $100 \%$ & $100 \%$ & $80 \%$ & 6 & -- & -- & 3 \\
\hline Bakken & BK-3 & 0 & 291 & 11 & & $x$ & & -- & $100 \%$ & $100 \%$ & $80 \%$ & 5 & -- & -- & 5 \\
\hline & BK-4 & G & 531 & 18 & $\mathrm{X}$ & & & $100 \%$ & $100 \%$ & $100 \%$ & $60 \%$ & 6 & 9 & -- & 8 \\
\hline & BK-20 & 0 & 854 & 29 & & $x$ & & -- & $20 \%$ & $100 \%$ & $16 \%$ & 6 & 15 & 18 & -- \\
\hline
\end{tabular}

Fig. 59 illustrates the hindcasting concept. Fig. 60 and Fig. 61 show the hindcasting analysis of well M-8 using DCA and PN-DCA methods, respectively. In this case the modeling is 
done using 16 months of production only. Thus, the production for the next 24 months is estimated by applying DCA models and then is compared with actual production data. For this comparative analysis, eight DCA models (Arps, modified hyperbolic, SEPD, MSE, Duong, modified Duong, PLE and LGM) and five pressure normalization (PN) techniques have been implemented [pressure-normalized DCA (PN-DCA), pseudopressure-normalized DCA $(\triangle \Psi N-$ DCA), square pressure-normalized DCA (SPN-DCA), and pressure corrected DCA ( $\triangle P F-D C A)]$, in addition to the traditional decline curve analysis (DCA). A comprehensive description of the DCA models is presented in Section 2, while PN techniques are described in Section 4.4. The harmonic decline model (harmonic decline of PNR vs. cumulative production), proposed by Anderson, D.M. et al. (2012), has also been included in the comparison.

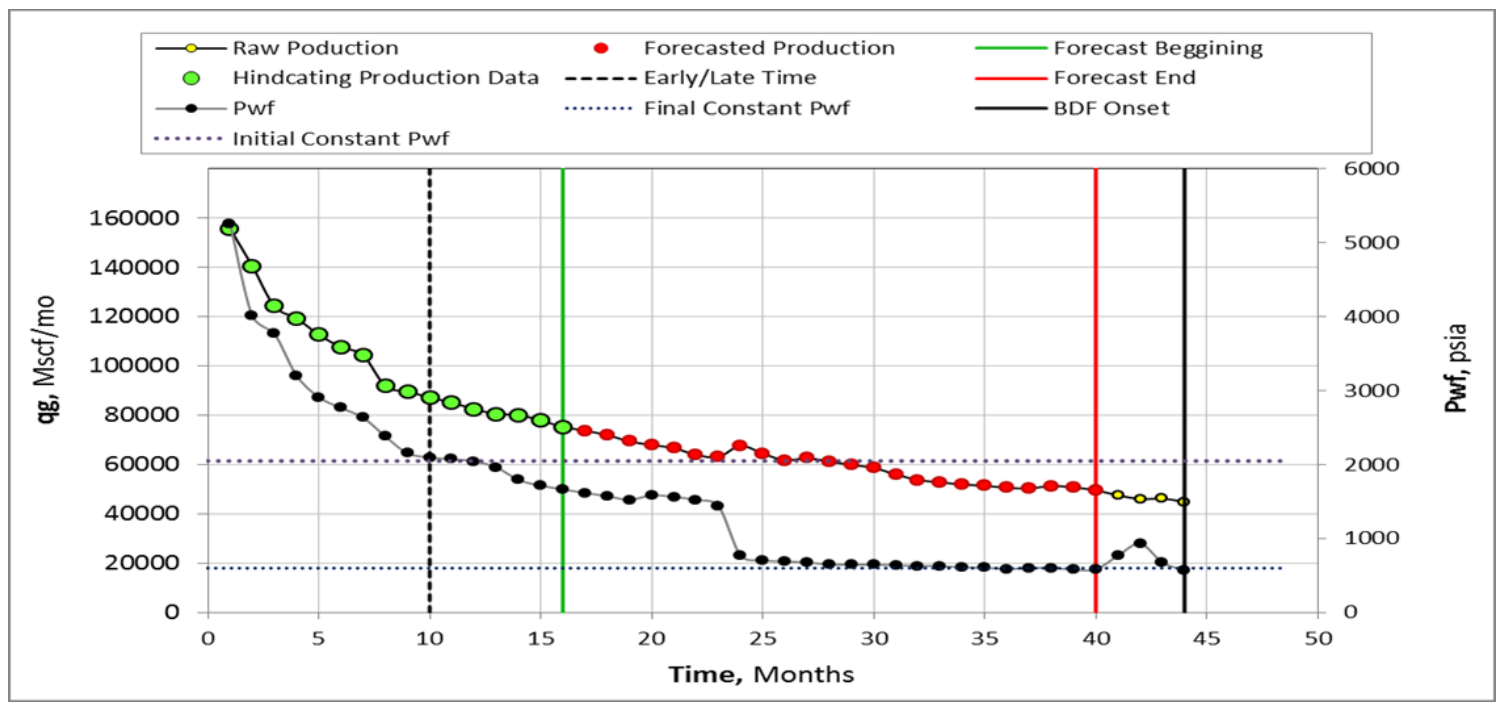

Fig. 59-Example of production data preparation for a hindcasting analysis. Green points indicate the portion of real data that is used to match the DCA models. Remaining red points correspond to the production data that will be compare with the predicted decline curve. 


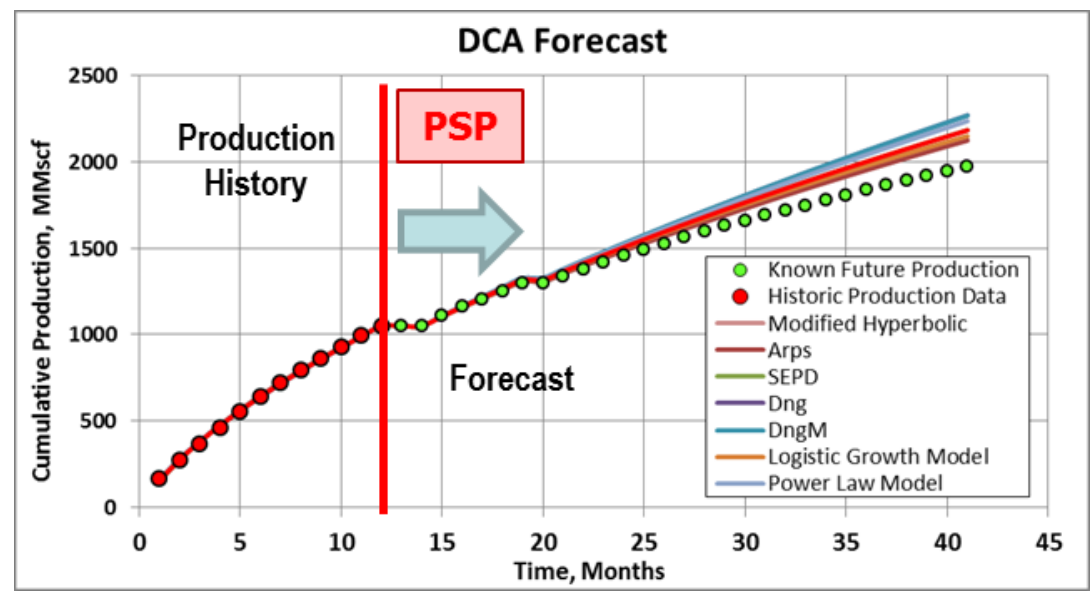

Fig. 60-Hindcasting analysis of DCA models for well M-8. Red points indicate the portion of production history used to match the DCA models. Green points are the portion of the production history used to compare the accuracy of the forecasts provided by the DCA models. The red line represents the prediction start point (PSP).

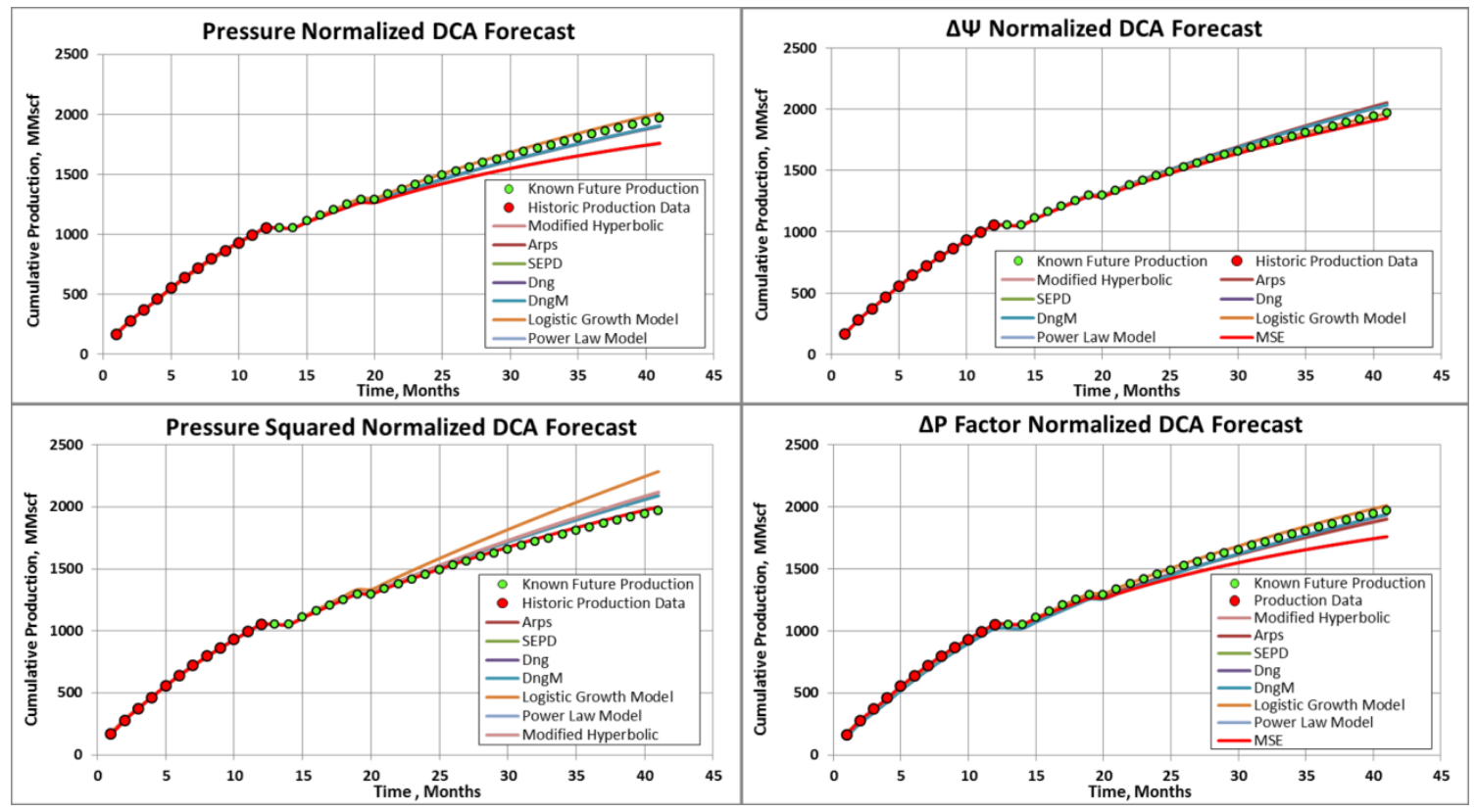

Fig. 61-Application of hindcasting with PN-DCA for well M-8. 


\subsubsection{Analysis of Results - General Scenario (All Wells included)}

The first level of comparison is a general scenario where all the wells are included along with all the predictions, DCA models and techniques. In total, 4272 hindcasts comprise the entire set of outputs included in this scenario. Further comparison will be presented to analyze hindcast results for specific scenarios depending on the type of fluid. Main observations about the general scenario are presented below:

- Overall, the traditional DCA technique-based on production rates onlyprovided the most accurate approach (lower mean percent error) when all DCA models' outputs are averaged (See top line of Table 16).

- Although all methods showed similar values of standard deviation, the lower value was produced by the harmonic decline method $(\mathrm{HE})$, meaning that this may generally provide a more precise output.

- All of the techniques generally provide conservative results (negative mean percent error). However, the traditional DCA gives the least conservative results.

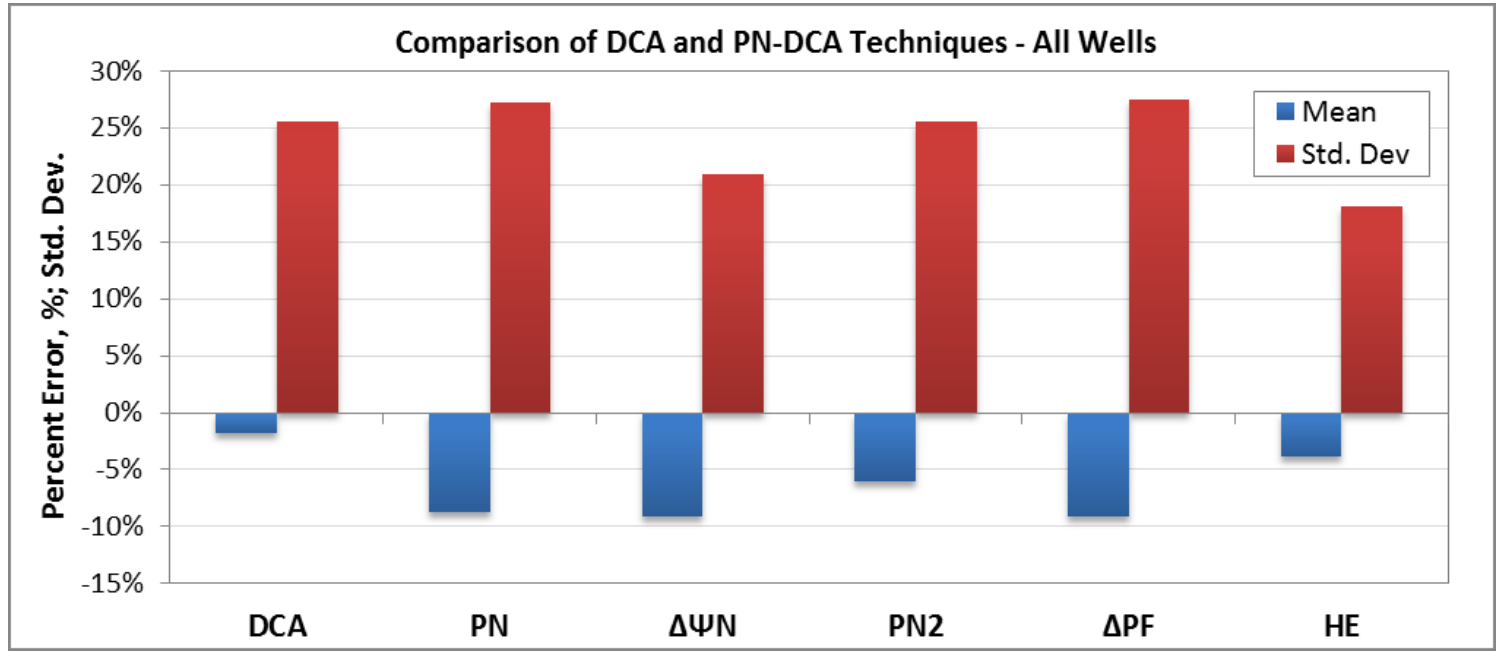

Fig. 62-Results of the comparative analysis, all wells included. 

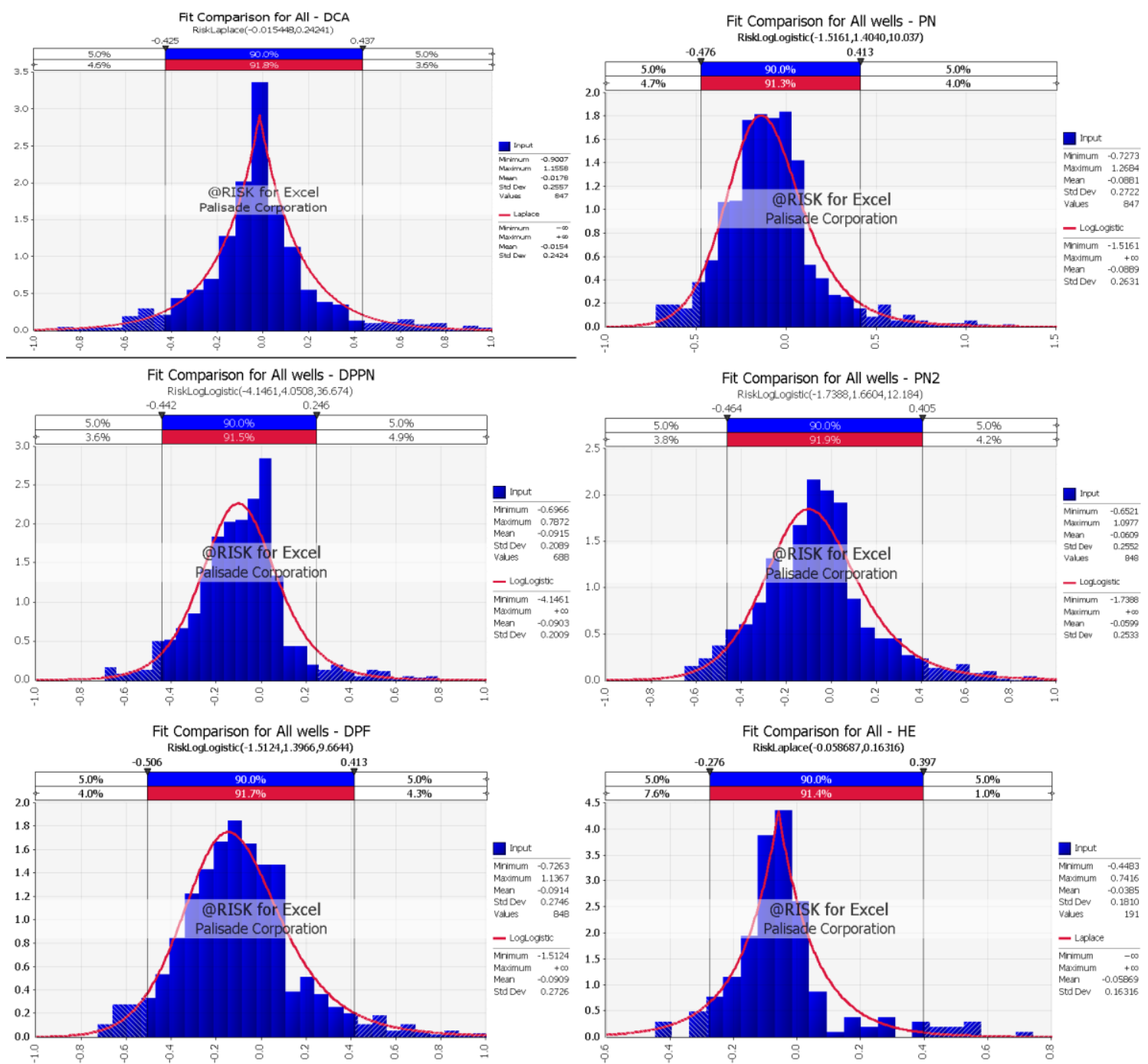

Fig. 63-Histogram of results (percent error) for each DCA technique in the general scenario (all wells included). The red line on each plot corresponds to the probabilistic function that best fits the results. This probabilistic function is presented only for visualization purposes, because comparative analysis is based on calculated data.

Results for each DCA technique (DCA, PN, $\triangle \Psi N, \mathrm{SPN}$, and $\triangle \mathrm{PF}$ ) are presented in Table 16 and Fig. 62 through Fig. 64. 


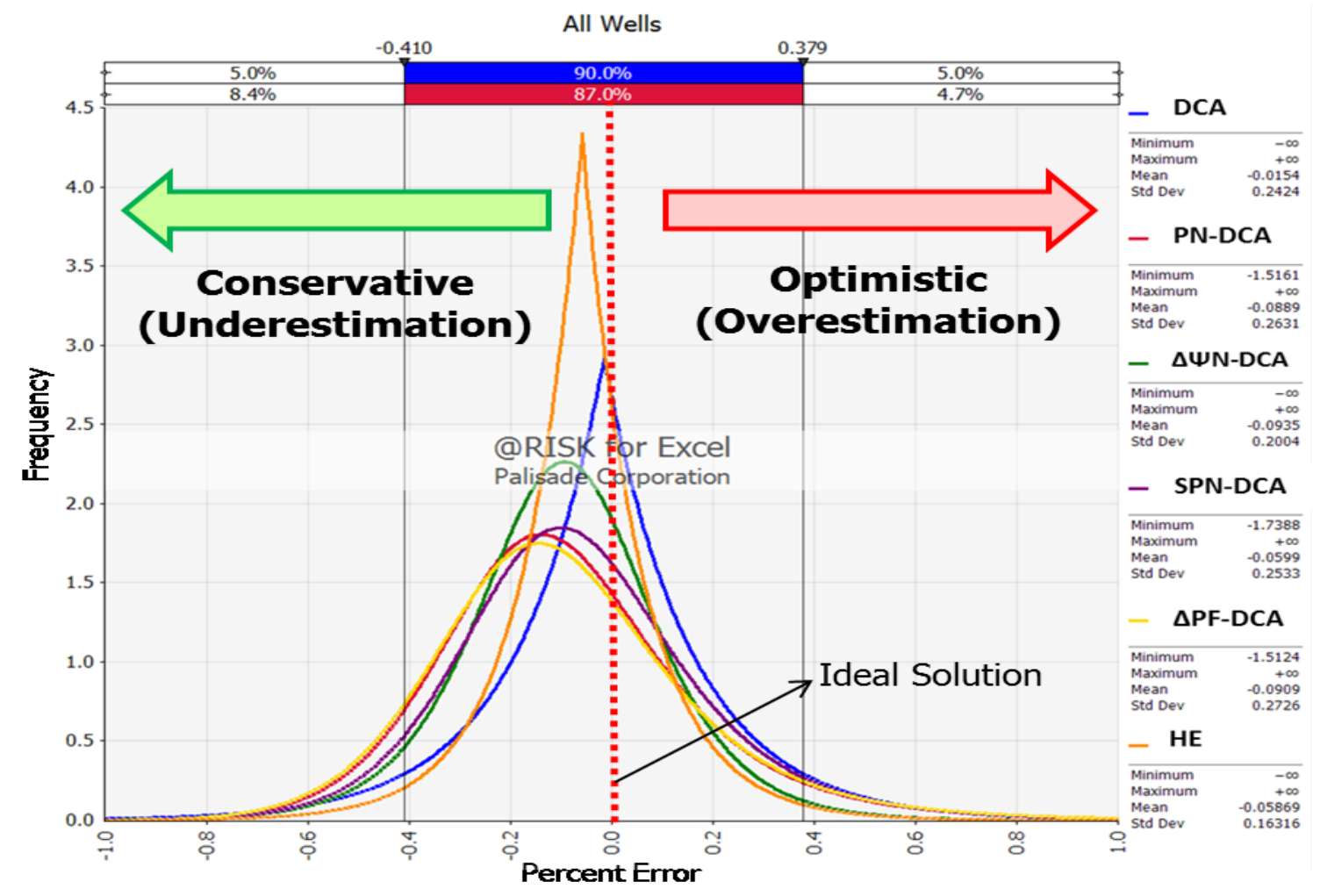

Fig. 64-Comparison of probabilistic functions representing the average tendency of the results for the general scenario (all wells included).

The results of the comparative analysis between the DCA models are presented in Table 17 and Fig. 65. The green cells indicate the best DCA technique (traditional DCA or Pressure Normalize DCA) for each particular DCA model (e.g., Arps, SEPD, Duong). Specific observations about the DCA models and techniques are discussed below:

- Arps' models, as well as Modified Hyperbolic and Modified SEPD, provided better estimations if applied with the traditional DCA method.

- SEPD, Modified Duong, and Power Law models provided better estimations when applied with the SPN method.

- LGM and Duong's model provided better results if applied with $\Delta \Psi N$ and PN methods, respectively. 
- If the outputs of all DCA techniques are averaged (See bottom line of Table 17), the Modified Duong's model may provide the best average solution (Average of mean percent errors: $0.4 \%)$.

- The forecasting capacity of Duong and modified Duong was optimized by applying the PN-DCA techniques.

- SEPD and MSE produced the most conservative estimations (lower percent error).

- In general, the PN-DCA techniques tend to generate more conservative estimations than the traditional DCA technique (See Fig. 65).

Table 16-Results of the comparative analysis of DCA techniques (general, oil, and gas scenarios).

\begin{tabular}{|c|c|c|c|c|c|c|c|}
\hline All & DCA & PN & $\Delta \Psi N$ & PN2 & $\Delta \mathrm{PF}$ & HE & Average \\
\hline Mean & $-1.8 \%$ & $-8.8 \%$ & $-9.2 \%$ & $-6.1 \%$ & $-9.1 \%$ & $-3.9 \%$ & $-6.5 \%$ \\
\hline Std. Dev & 0.26 & 0.27 & 0.21 & 0.26 & 0.27 & 0.18 & 0.24 \\
\hline Minimum & $-90.1 \%$ & $-72.7 \%$ & $-69.7 \%$ & $-65.2 \%$ & $-72.6 \%$ & $-44.8 \%$ & $-69.2 \%$ \\
\hline Maximum & $115.6 \%$ & $126.8 \%$ & $78.7 \%$ & $109.8 \%$ & $113.7 \%$ & $74.2 \%$ & $103.1 \%$ \\
\hline Gas & DCA & PN & $\Delta \Psi N$ & PN2 & $\Delta \mathrm{PF}$ & HE & Average \\
\hline Mean & $0.0 \%$ & $-11.1 \%$ & $-9.1 \%$ & $-6.8 \%$ & $-11.3 \%$ & $-4.2 \%$ & $-7.1 \%$ \\
\hline Std Dev & 0.23 & 0.22 & 0.21 & 0.24 & 0.22 & 0.18 & 0.22 \\
\hline Minimum & $-90.1 \%$ & $-68.5 \%$ & $-69.7 \%$ & $-65.2 \%$ & $-67.7 \%$ & $-44.8 \%$ & $-67.7 \%$ \\
\hline Maximum & $101.4 \%$ & $126.8 \%$ & $78.7 \%$ & $109.8 \%$ & $95.1 \%$ & $55.7 \%$ & $94.6 \%$ \\
\hline Oil & DCA & PN & $\Delta \Psi N$ & PN2 & $\Delta \mathrm{PF}$ & HE & Average \\
\hline Mean & $-9.3 \%$ & $0.9 \%$ & -- & $-3.1 \%$ & $0.1 \%$ & $-0.2 \%$ & $-2.3 \%$ \\
\hline Std Dev & 0.34 & 0.42 & -- & 0.33 & 0.42 & 0.22 & 0.34 \\
\hline Minimum & $-85.2 \%$ & $-72.7 \%$ & -- & $-63.9 \%$ & $-72.6 \%$ & $-20.3 \%$ & $-63.0 \%$ \\
\hline Maximum & $115.6 \%$ & $110.2 \%$ & -- & $94.5 \%$ & $113.7 \%$ & $74.2 \%$ & $101.6 \%$ \\
\hline
\end{tabular}


Table 17-Summary of results (mean percent error) for the general comparative analysis (all wells included).

\begin{tabular}{|c|c|c|c|c|c|c|c|c|c|}
\hline All & Arps & MHyp & SEPD & MSE & DNG & MDng & LGM & PLE & HE \\
\hline DCA & $-2.3 \%$ & $-2.5 \%$ & $-16.1 \%$ & $-13.2 \%$ & $10.4 \%$ & $6.7 \%$ & $9.9 \%$ & $-7.2 \%$ & \\
\hline PN & $-7.7 \%$ & $-9.2 \%$ & $-25.8 \%$ & $-24.6 \%$ & $1.2 \%$ & $-1.9 \%$ & $12.0 \%$ & $-14.3 \%$ & $-3.8 \%$ \\
\hline $\boldsymbol{\Delta} \boldsymbol{\Psi N}$ & $-5.2 \%$ & $-6.3 \%$ & $-22.7 \%$ & $-23.3 \%$ & $-3.2 \%$ & $-4.6 \%$ & $3.4 \%$ & $-11.2 \%$ & $-3.9 \%$ \\
\hline SPN & $-17.2 \%$ & $-16.4 \%$ & $-15.5 \%$ & $-14.6 \%$ & $2.3 \%$ & $-0.3 \%$ & $12.2 \%$ & $0.7 \%$ & \\
\hline $\boldsymbol{\Delta P F}$ & $-8.9 \%$ & $-10.3 \%$ & $-25.8 \%$ & $-24.4 \%$ & $5.0 \%$ & $2.0 \%$ & $9.0 \%$ & $-19.7 \%$ & \\
\hline Average & $-8.3 \%$ & $-8.9 \%$ & $-21.2 \%$ & $-20.0 \%$ & $3.1 \%$ & $0.4 \%$ & $9.3 \%$ & $-10.3 \%$ & $-3.8 \%$ \\
\hline
\end{tabular}

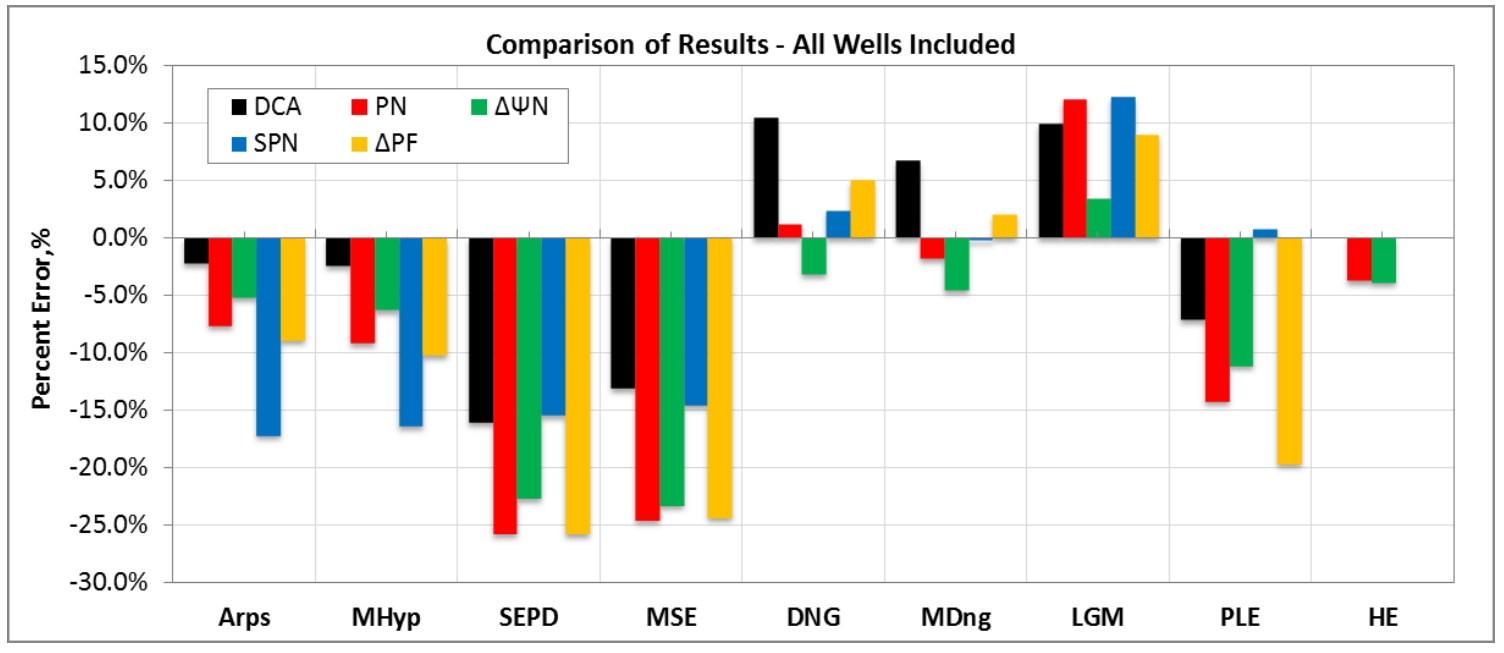

Fig. 65-Comparison of results for all DCA models (All wells included).

\subsubsection{Analysis of Results - Gas Scenario}

All gas wells have been grouped in this scenario. In total, 3612 hindcasts comprise the entire set of outputs included in this scenario. Most important observations are presented below:

- In general, the traditional DCA technique provided the best average result (mean percent error: 0.0\%) for the whole scenario (Fig. 66). 
- The traditional DCA technique provided better results for Arps, Modified Hyperbolic, SEPD and MSE models.

- The forecasting capacity of Duong and Modified Duong significantly improves if applied with the PN-DCA techniques-especially with SPN-instead of the traditional DCA.

- LGM methods worked better with the $\triangle \mathrm{PF}$ technique, while PLE yielded better results with the SPN method. See Table 18 and Fig. 67 for more details.

- If the average of all techniques is considered, Duong's model may provide the best approach (Average: 0.3\%).

- Just like in the general scenario (all wells included), PN-DCA techniques tend to generate more conservative estimations than the traditional DCA technique (See Fig. 67).

- On average, SEPD and MSE produced the most conservative estimations (lower percent error).

- An important observation is that in all cases $\Delta \Psi \mathrm{N}$ provided better outputs than the simple PN technique, which proves that more representative results may be obtained if the pressure-dependent properties of gas are included in production analysis by using pseudovariables (pseudopressures, pseudotime, and material balance pseudotime).

Table 18-Summary of results (mean percent error) of the comparative analysis for gas wells.

\begin{tabular}{|c|r|r|r|r|r|r|r|r|r|}
\hline Gas & Arps & Mhyp & SEPD & MSE & Duong & MDng & LGM & PLE & HE \\
\hline DCA & $0.8 \%$ & $0.2 \%$ & $-11.0 \%$ & $-10.6 \%$ & $9.6 \%$ & $8.8 \%$ & $5.9 \%$ & $-3.8 \%$ & \\
\hline PN & $-7.3 \%$ & $-8.7 \%$ & $-25.4 \%$ & $-26.2 \%$ & $-5.2 \%$ & $-7.1 \%$ & $5.0 \%$ & $-13.5 \%$ & $-4.6 \%$ \\
\hline$\Delta \Psi \mathbf{N}$ & $-5.2 \%$ & $-6.3 \%$ & $-22.7 \%$ & $-23.3 \%$ & $-3.2 \%$ & $-4.6 \%$ & $3.4 \%$ & $-11.2 \%$ & $-3.9 \%$ \\
\hline SPN & $-15.7 \%$ & $-16.5 \%$ & $-15.0 \%$ & $-15.7 \%$ & $1.0 \%$ & $-0.2 \%$ & $9.1 \%$ & $-1.2 \%$ & \\
\hline$\Delta$ PF & $-8.4 \%$ & $-9.8 \%$ & $-25.5 \%$ & $-26.3 \%$ & $-0.5 \%$ & $-2.3 \%$ & $1.7 \%$ & $-19.0 \%$ & \\
\hline Average & $-7.2 \%$ & $-8.2 \%$ & $-19.9 \%$ & $-20.4 \%$ & $0.3 \%$ & $-1.1 \%$ & $5.0 \%$ & $-9.8 \%$ & $-4.2 \%$ \\
\hline
\end{tabular}




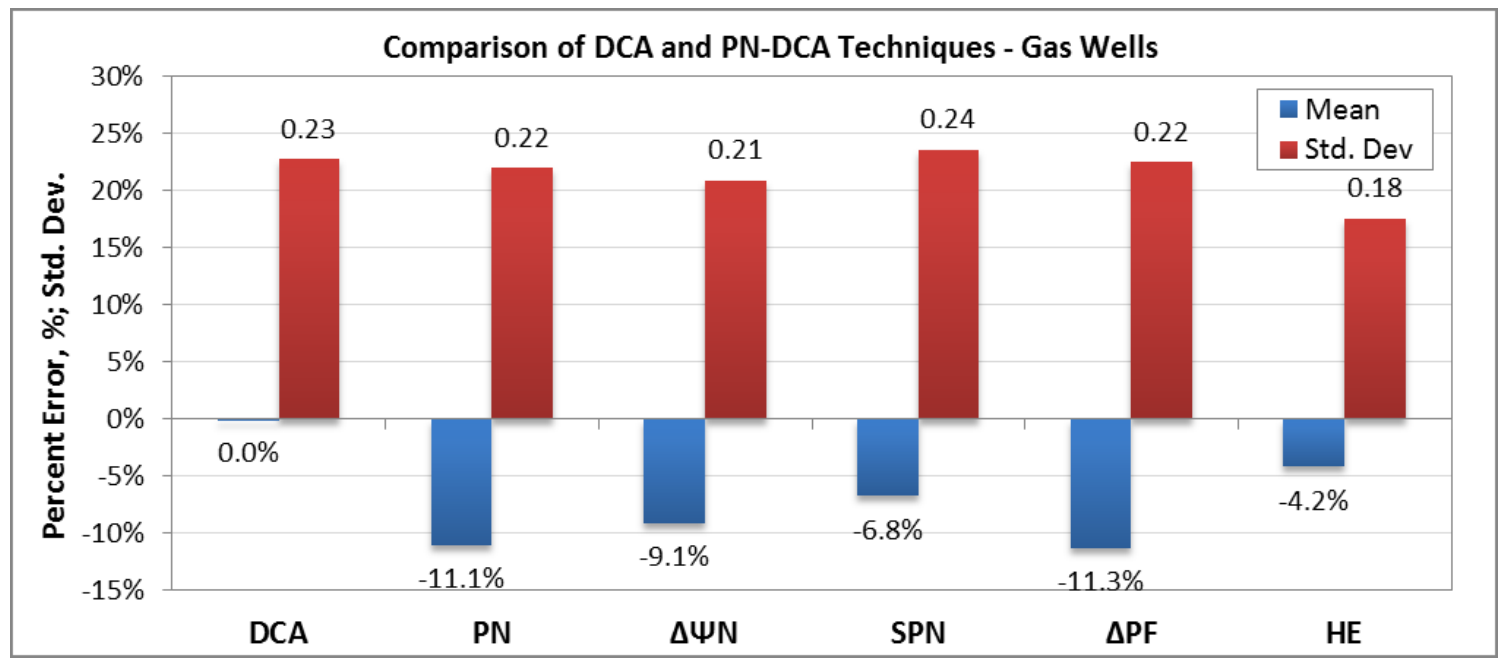

Fig. 66-Results of the comparative analysis, only gas wells included.

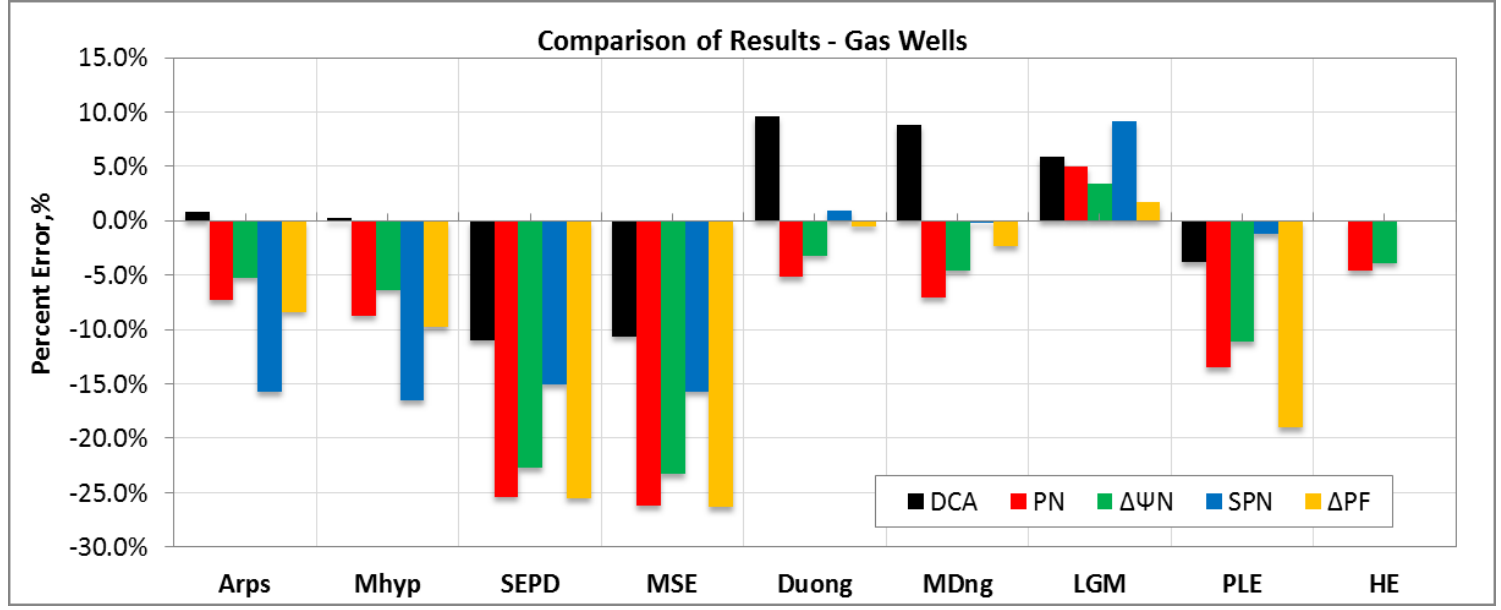

Fig. 67-Comparison of results for gas wells only.

Considering that $71 \%$ of the wells used in this analysis are gas producers, it is not strange to find similar observations between the general and the gas well scenarios. 


\subsubsection{Analysis of Results - Oil Scenario}

All oil wells have been grouped in this scenario. In total, 660 hindcasts comprise the entire set of outputs included in this scenario. Most important observations are presented below.

Table 19-Summary of results (mean percent error) of the comparative analysis for oil wells.

\begin{tabular}{|c|r|r|r|r|r|r|r|r|c|}
\hline Oil & Arps & Mhyp & SEPD & MSE & Duong & MDng & LGM & PLE & HE \\
\hline DCA & $-15.3 \%$ & $-13.9 \%$ & $-38.0 \%$ & $-24.0 \%$ & $14.1 \%$ & $-2.6 \%$ & $26.6 \%$ & $-21.4 \%$ & \\
\hline PN & $-9.3 \%$ & $-11.3 \%$ & $-27.7 \%$ & $-17.6 \%$ & $28.5 \%$ & $20.6 \%$ & $41.6 \%$ & $-17.4 \%$ & $-0.2 \%$ \\
\hline SPN & $-23.5 \%$ & $-15.9 \%$ & $-17.4 \%$ & $-9.9 \%$ & $8.1 \%$ & $-0.5 \%$ & $25.3 \%$ & $9.1 \%$ & \\
\hline DPF & $-11.4 \%$ & $-12.1 \%$ & $-27.1 \%$ & $-16.0 \%$ & $28.4 \%$ & $20.6 \%$ & $40.4 \%$ & $-22.4 \%$ & \\
\hline Average & $-14.9 \%$ & $-13.3 \%$ & $-27.5 \%$ & $-16.9 \%$ & $19.8 \%$ & $9.5 \%$ & $33.5 \%$ & $-13.0 \%$ & $-0.2 \%$ \\
\hline
\end{tabular}

- Overall, the PN-DCA techniques provided much better results than the traditional DCA method.

- If the average of all DCA models is considered, the $\triangle \mathrm{PF}$ technique provided the most accurate output (lower mean percent error: $0.1 \%$ ) for this scenario (See Fig. 68).

- Arps' models yielded better estimations when applied with the PN-DCA technique, while the other models worked better with the SPN technique (See Fig. 69 and Table 19).

- If the average of all techniques is considered, modified Duong's model may provide the best approach (Average: 9.5\%) in comparison with the other DCA models.

- The best approximation was produced by the Harmonic Decline (HE) model (mean percent error: $-0.2 \%$ ), which also has the lowest standard deviation, and by Modified Duong with the SPN-DCA technique (See). 
- In general, Arps, SEPD, MSE and PLE showed a tendency to underestimate forecasts (conservative), while the other models showed a more optimistic behavior.

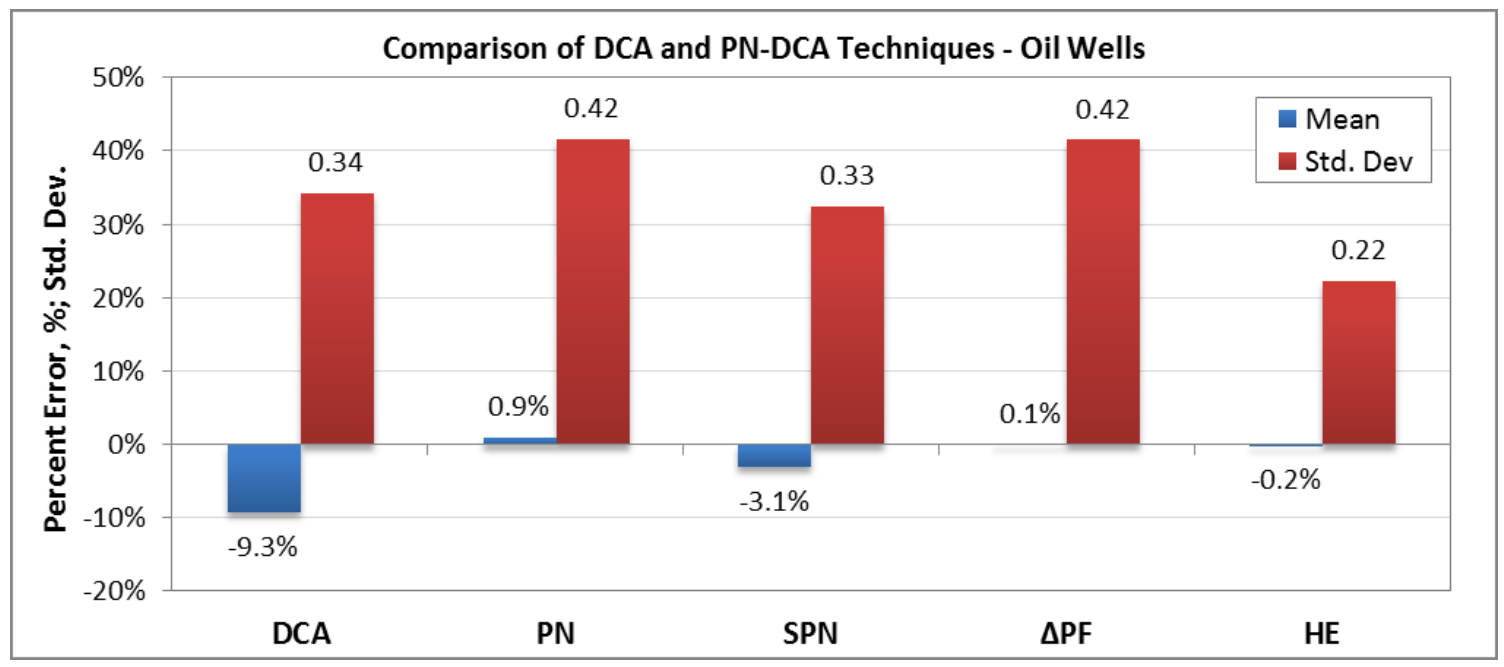

Fig. 68-Results of the comparative analysis, only oil wells included.

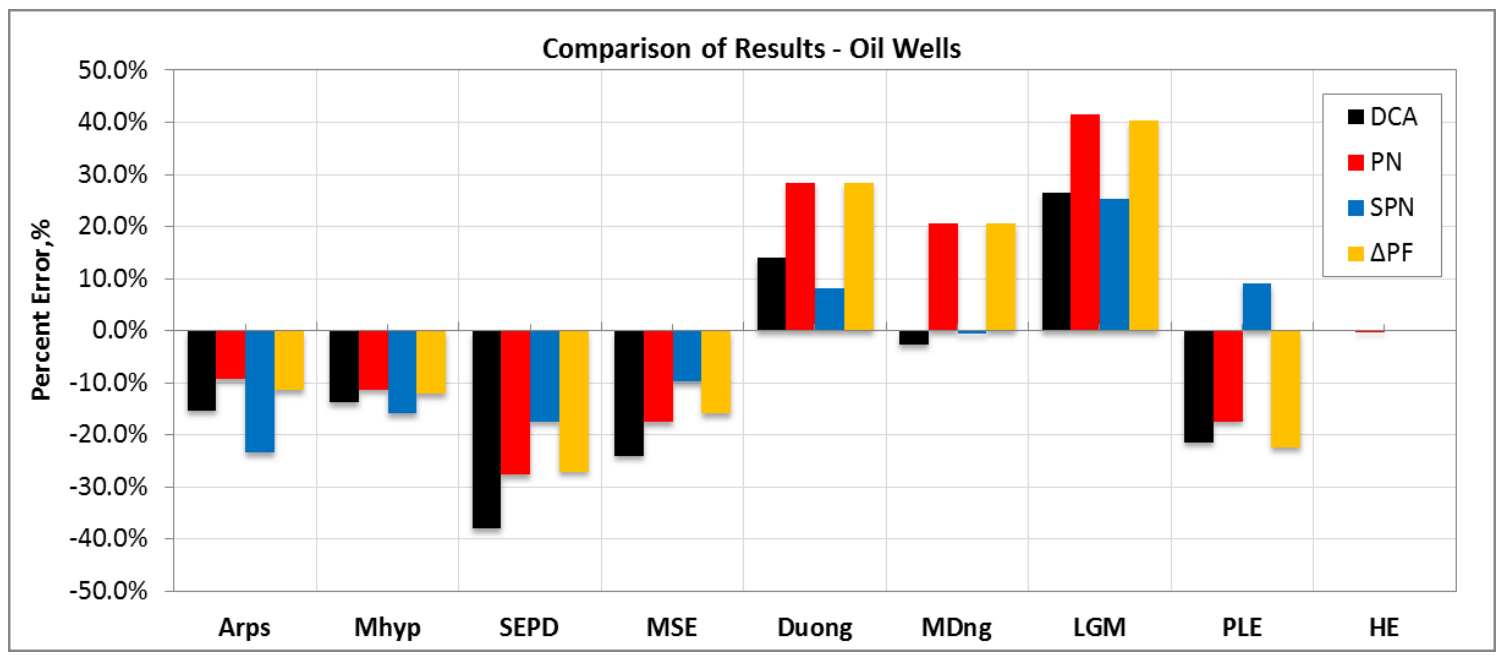

Fig. 69-Comparison of results for oil wells only. 


\subsubsection{Analysis of Results by Play}

In this section, the results are presented for each play in separate.

Table 20-Summary of results (mean percent error) for each play.

\begin{tabular}{|c|c|c|c|c|c|c|c|c|c|c|}
\hline Woodford & Arps & MHyp & SEPD & MSE & DNG & MDng & LGM & PLE & HE & $\begin{array}{l}\text { Average DCA } \\
\text { and PN-DCA }\end{array}$ \\
\hline DCA & $-3.6 \%$ & $-3.6 \%$ & $-12.2 \%$ & $-12.2 \%$ & $2.7 \%$ & $2.7 \%$ & $-4.1 \%$ & $-6.0 \%$ & & $-4.5 \%$ \\
\hline PN & $-3.6 \%$ & $-3.6 \%$ & $-20.5 \%$ & $-20.5 \%$ & $-3.5 \%$ & $-3.5 \%$ & $3.5 \%$ & $-7.7 \%$ & $-8.8 \%$ & $-7.4 \%$ \\
\hline$\Delta \Psi N$ & $-5.2 \%$ & $-5.2 \%$ & $-22.3 \%$ & $-22.3 \%$ & $-4.5 \%$ & $-4.5 \%$ & $0.0 \%$ & $-9.0 \%$ & $-10.2 \%$ & $-9.1 \%$ \\
\hline SPN & $-33.8 \%$ & $-33.8 \%$ & $-12.8 \%$ & $-12.8 \%$ & $-0.1 \%$ & $-0.1 \%$ & $4.9 \%$ & $-4.4 \%$ & & $-11.6 \%$ \\
\hline$\Delta \mathrm{PF}$ & $-3.7 \%$ & $-3.7 \%$ & $-21.2 \%$ & $-21.2 \%$ & $2.9 \%$ & $2.9 \%$ & $-0.9 \%$ & $-13.9 \%$ & & $-7.3 \%$ \\
\hline Average & $-10.0 \%$ & $-10.0 \%$ & $-17.8 \%$ & $-17.8 \%$ & $-0.5 \%$ & $-0.5 \%$ & $0.7 \%$ & $-8.2 \%$ & $-9.5 \%$ & $-8.0 \%$ \\
\hline Eagle Ford & Arps & Mhyp & SEPD & MSE & Duong & MDng & LGM & PLE & HE & $\begin{array}{l}\text { Average DCA } \\
\text { and PN-DCA }\end{array}$ \\
\hline DCA & $-13.7 \%$ & $-14.3 \%$ & $-41.2 \%$ & $-27.9 \%$ & $18.0 \%$ & $-0.1 \%$ & $27.6 \%$ & $-17.6 \%$ & & $-8.7 \%$ \\
\hline PN & $-1.0 \%$ & $-7.9 \%$ & $-26.0 \%$ & $-19.2 \%$ & $34.0 \%$ & $25.8 \%$ & $47.7 \%$ & $-14.2 \%$ & $3.6 \%$ & $4.9 \%$ \\
\hline SPN & $-25.1 \%$ & $-16.5 \%$ & $-12.8 \%$ & $-8.4 \%$ & $18.8 \%$ & $9.8 \%$ & $28.1 \%$ & $13.3 \%$ & & $0.9 \%$ \\
\hline$\Delta \mathrm{PF}$ & $-6.0 \%$ & $-12.5 \%$ & $-24.8 \%$ & $-18.0 \%$ & $34.0 \%$ & $25.8 \%$ & $47.1 \%$ & $-18.8 \%$ & & $3.3 \%$ \\
\hline Average & $-11.4 \%$ & $-12.8 \%$ & $-26.2 \%$ & $-18.4 \%$ & $26.2 \%$ & $15.3 \%$ & $37.6 \%$ & $-9.4 \%$ & $3.6 \%$ & $0.1 \%$ \\
\hline Bakken & Arps & Mhyp & SEPD & MSE & Duong & MDng & LGM & PLE & HE & $\begin{array}{l}\text { Average DCA } \\
\text { and PN-DCA }\end{array}$ \\
\hline DCA & $-19.2 \%$ & $-12.8 \%$ & $-30.5 \%$ & $-14.9 \%$ & $5.0 \%$ & $-8.5 \%$ & $24.1 \%$ & $-30.3 \%$ & & $-10.9 \%$ \\
\hline PN & $-28.8 \%$ & $-19.3 \%$ & $-31.7 \%$ & $-13.8 \%$ & $15.5 \%$ & $8.6 \%$ & $27.2 \%$ & $-24.9 \%$ & $-9.3 \%$ & $-8.4 \%$ \\
\hline SPN & $-19.7 \%$ & $-14.7 \%$ & $-28.3 \%$ & $-13.2 \%$ & $-16.8 \%$ & $-24.5 \%$ & $18.9 \%$ & $-0.5 \%$ & & $-12.3 \%$ \\
\hline$\Delta \mathrm{PF}$ & $-23.9 \%$ & $-11.4 \%$ & $-32.2 \%$ & $-11.2 \%$ & $15.5 \%$ & $8.6 \%$ & $24.7 \%$ & $-30.8 \%$ & & $-7.6 \%$ \\
\hline Average & $-22.9 \%$ & $-14.6 \%$ & $-30.7 \%$ & $-13.3 \%$ & $4.8 \%$ & $-3.9 \%$ & $23.7 \%$ & $-21.6 \%$ & $-9.3 \%$ & $-9.8 \%$ \\
\hline Marcellus & Arps & Mhyp & SEPD & MSE & Duong & MDng & LGM & PLE & HE & $\begin{array}{l}\text { Average DCA } \\
\text { and PN-DCA }\end{array}$ \\
\hline DCA & $2.4 \%$ & $1.3 \%$ & $-7.7 \%$ & $-8.2 \%$ & $12.6 \%$ & $11.6 \%$ & $10.0 \%$ & $-0.8 \%$ & & $2.6 \%$ \\
\hline PN & $-9.5 \%$ & $-11.2 \%$ & $-30.0 \%$ & $-31.0 \%$ & $-10.6 \%$ & $-12.4 \%$ & $-3.0 \%$ & $-16.4 \%$ & $-1.1 \%$ & $-15.5 \%$ \\
\hline$\Delta \Psi N$ & $-4.0 \%$ & $-5.3 \%$ & $-23.4 \%$ & $-24.0 \%$ & $-5.2 \%$ & $-6.4 \%$ & $-1.0 \%$ & $-10.7 \%$ & $1.3 \%$ & $-10.0 \%$ \\
\hline SPN & $-1.9 \%$ & $-2.9 \%$ & $-19.0 \%$ & $-19.6 \%$ & $-0.7 \%$ & $-1.9 \%$ & $5.0 \%$ & $-6.0 \%$ & & $-5.9 \%$ \\
\hline$\Delta \mathrm{PF}$ & $-11.7 \%$ & $-13.3 \%$ & $-29.6 \%$ & $-30.6 \%$ & $-7.4 \%$ & $-9.0 \%$ & $-4.1 \%$ & $-22.5 \%$ & & $-16.0 \%$ \\
\hline Average & $-4.9 \%$ & $-6.3 \%$ & $-21.9 \%$ & $-22.7 \%$ & $-2.3 \%$ & $-3.6 \%$ & $1.4 \%$ & $-11.3 \%$ & $0.1 \%$ & $-9.0 \%$ \\
\hline
\end{tabular}



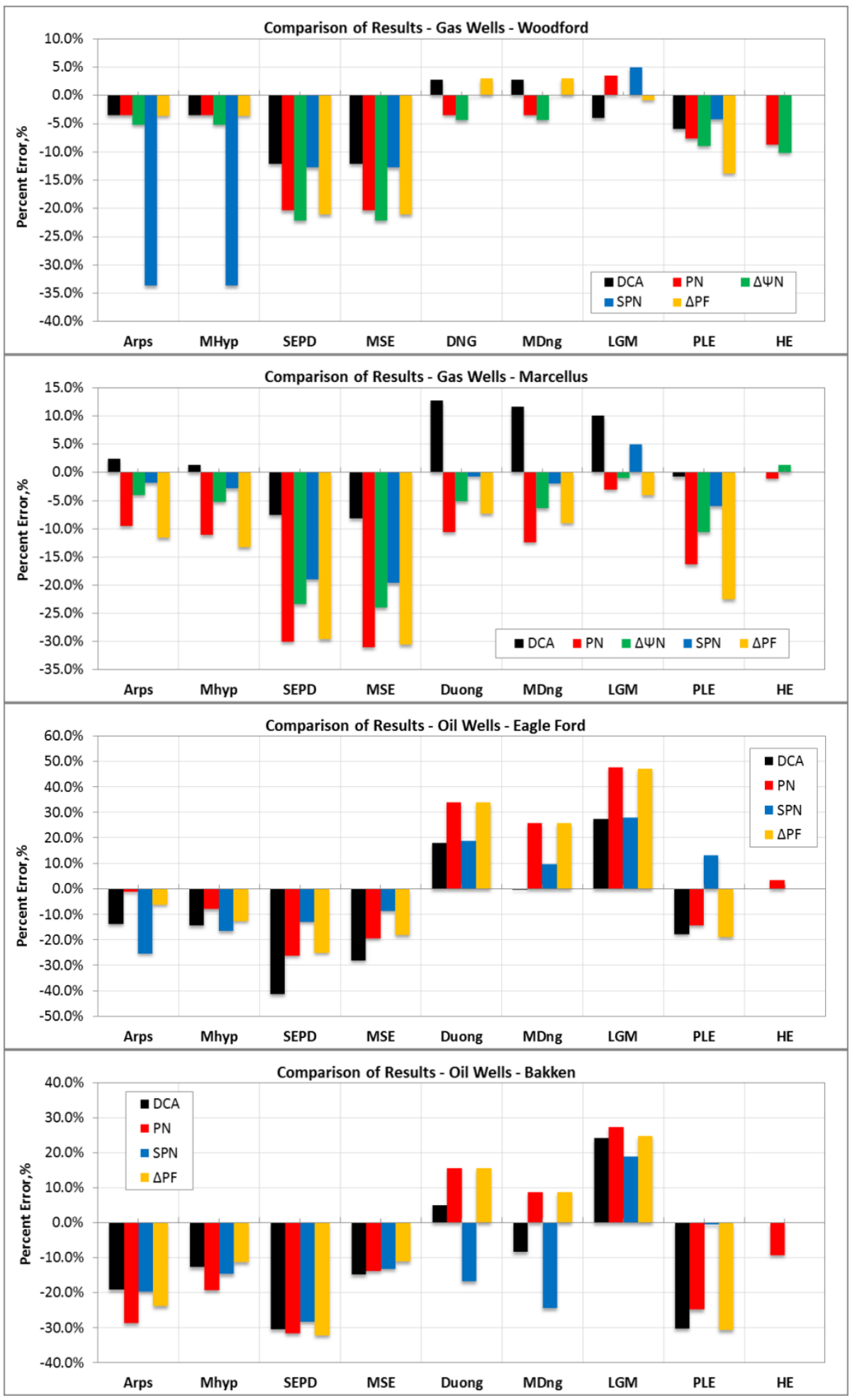

Fig. 70-Comparison of results for each play. 
Overall, similar trends may be observed between the gas plays, as well as between the two oil plays (See Fig. 70). Therefore, general observations are similar to those mentioned in the two previous sections for oil and gas scenarios. It means that the play by play inspection confirmed the observation deducted from the analysis by fluid type (gas and oil scenarios). Table 20 presents a summary of results obtained for each play. Note that the green cells show the best technique for each DCA model, the red cells indicate the best DCA model in general (considering an average of all techniques), and the yellow cells point out the worst DCA model.

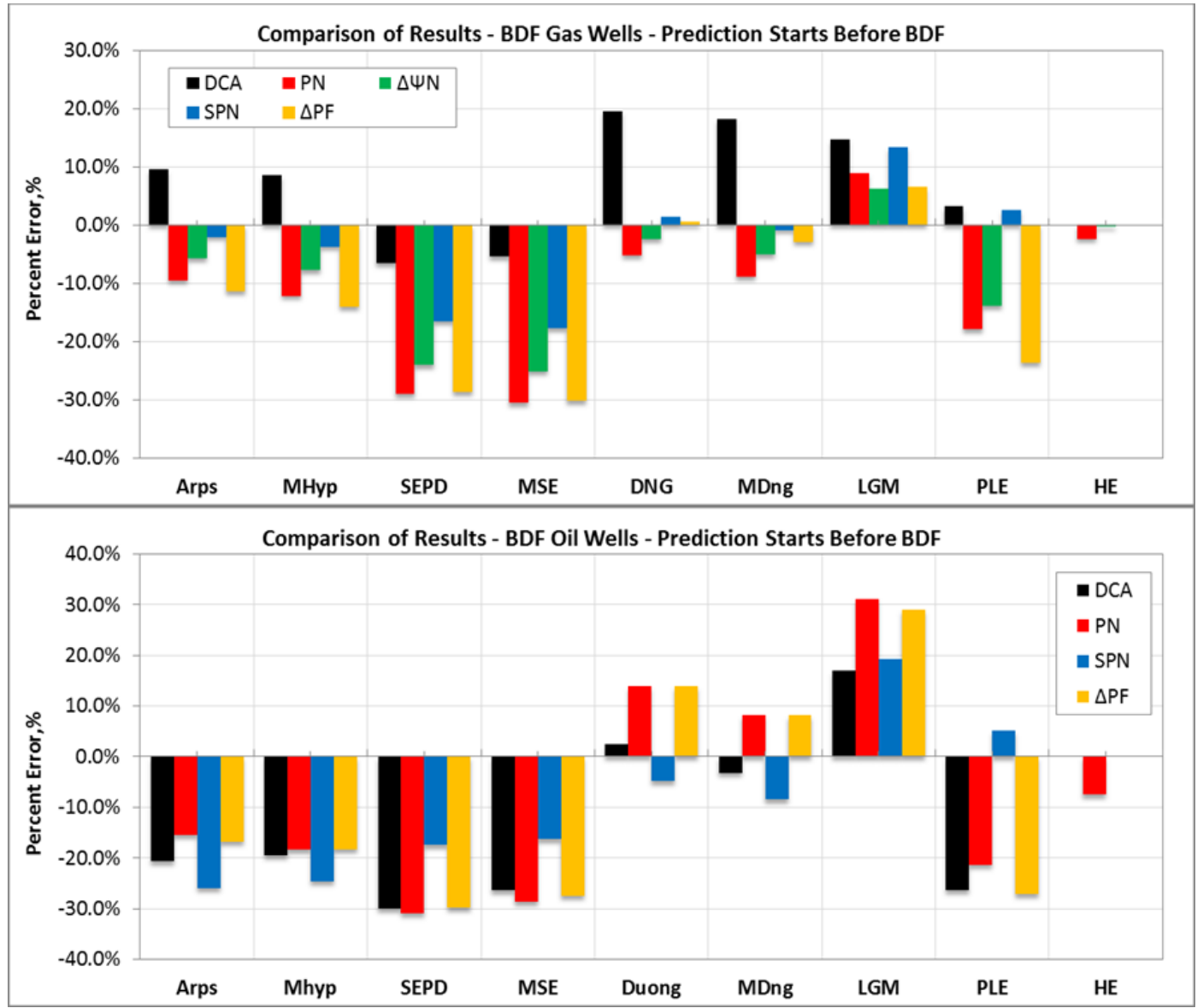

Fig. 71-Comparison of results for wells on BDF. 


\subsubsection{Analysis of Results - Wells on Boundary-Dominated Flow}

All wells exhibiting the BDF signature have been grouped in this scenario. In total, 1941 predictions comprise the entire set of outputs included in this scenario (1512 correspond to gas wells, while 429 correspond to oil wells). The prediction start points are before the BDF onsets. According to this classification, DCA models and techniques display similar trends for oil and gas wells (See Table 21 and Fig. 71). The most important observations are presented below:

- Dual models (Modified Hyperbolic, MSE, and Modified Duong), which are specially designed to better switch from transient to BDF, produced slightly better results in comparison with their parent models (Arps, SEPD and Duong).

- Consistently, SEPD and MSE generated the most conservative results.

- With few exceptions, Arps, SEPD, MSE and PLE are generally conservative models (average of all techniques).

- For gas wells, the traditional DCA technique yielded very optimistic results for Arps, Duong, Modified Duong and LGM models. However, applying Pressure Normalization techniques reduced the overestimation tendency of these models and generally provided more conservative estimations.

- On average, Duong and modified Duong models generated more accurate forecasts for gas wells if applied with Pressure Normalization techniques. However, for oil wells the performance of these two models was better if applied with the traditional DCA techniques.

- LGM tends to be optimistic, even if Pressure Normalization techniques are used.

- The Harmonic Extrapolation (HE) method presented attractive results. 
Table 21-Summary of results (mean percent error) for wells exhibiting BDF.

\begin{tabular}{|c|c|c|c|c|c|c|c|c|c|c|}
\hline Gas - BDF & Arps & MHyp & SEPD & MSE & DNG & MDng & LGM & PLE & HE & $\begin{array}{l}\text { Average DCA } \\
\text { and PN-DCA }\end{array}$ \\
\hline DCA & $9.5 \%$ & $8.5 \%$ & $-6.6 \%$ & $-5.5 \%$ & $19.5 \%$ & $18.2 \%$ & $14.6 \%$ & $3.1 \%$ & & $7.7 \%$ \\
\hline PN & $-9.6 \%$ & $-12.2 \%$ & $-29.1 \%$ & $-30.7 \%$ & $-5.3 \%$ & $-9.0 \%$ & $8.9 \%$ & $-18.0 \%$ & $-2.4 \%$ & $-13.1 \%$ \\
\hline$\Delta \Psi N$ & $-5.8 \%$ & $-7.9 \%$ & $-24.2 \%$ & $-25.2 \%$ & $-2.5 \%$ & $-5.2 \%$ & $6.2 \%$ & $-14.0 \%$ & $-0.4 \%$ & $-9.8 \%$ \\
\hline SPN & $-2.1 \%$ & $-3.8 \%$ & $-16.6 \%$ & $-17.8 \%$ & $1.4 \%$ & $-0.9 \%$ & $13.3 \%$ & $2.6 \%$ & & $-3.0 \%$ \\
\hline$\Delta \mathrm{PF}$ & $-11.5 \%$ & $-14.2 \%$ & $-28.8 \%$ & $-30.3 \%$ & $0.6 \%$ & $-2.9 \%$ & $6.5 \%$ & $-23.8 \%$ & & $-13.1 \%$ \\
\hline Average & $-3.9 \%$ & $-5.9 \%$ & $-21.1 \%$ & $-21.9 \%$ & $2.7 \%$ & $0.0 \%$ & $9.9 \%$ & $-10.0 \%$ & $-1.4 \%$ & $-6.3 \%$ \\
\hline Oil - BDF & Arps & Mhyp & SEPD & MSE & Duong & MDng & LGM & PLE & HE & $\begin{array}{l}\text { Average DCA } \\
\text { and PN-DCA }\end{array}$ \\
\hline DCA & $-20.7 \%$ & $-19.5 \%$ & $-30.0 \%$ & $-26.4 \%$ & $2.4 \%$ & $-3.2 \%$ & $16.9 \%$ & $-26.4 \%$ & & $-13.4 \%$ \\
\hline PN & $-15.5 \%$ & $-18.4 \%$ & $-31.0 \%$ & $-28.7 \%$ & $13.8 \%$ & $8.1 \%$ & $31.1 \%$ & $-21.4 \%$ & $-7.6 \%$ & $-7.7 \%$ \\
\hline SPN & $-26.0 \%$ & $-24.6 \%$ & $-17.5 \%$ & $-16.3 \%$ & $-4.9 \%$ & $-8.5 \%$ & $19.2 \%$ & $5.1 \%$ & & $-9.2 \%$ \\
\hline$\Delta \mathrm{PF}$ & $-16.8 \%$ & $-18.3 \%$ & $-29.9 \%$ & $-27.5 \%$ & $13.8 \%$ & $8.1 \%$ & $28.9 \%$ & $-27.2 \%$ & & $-8.6 \%$ \\
\hline Average & $-19.8 \%$ & $-20.2 \%$ & $-27.1 \%$ & $-24.7 \%$ & $6.3 \%$ & $1.1 \%$ & $24.0 \%$ & $-17.5 \%$ & $-7.6 \%$ & $-9.7 \%$ \\
\hline
\end{tabular}

\subsubsection{Evolution of Forecasting Accuracy in Function of Amount of Production History}

The target of this section is to evaluate how the forecasting accuracy of DCA models and techniques evolve depending on the amount of production history available for the history match. It is expected that estimations accuracy improve as more production history is available.

Multiple prediction start times (hindcasting onset) have been selected for each well. Depending on the amount of production history, up to three predictions were performed on each well. Fig. 72 presents the average prediction start point (PSP) for each play. The first set of predictions (PSP 1) is indicated in red, while the second and third sets of predictions (PSP 2 and 3) correspond to the green and blue bars, respectively. Note that PSP consistently increase. There is not a third prediction scenario in Bakken because production the histories of these wells are short in comparison to the other plays. 


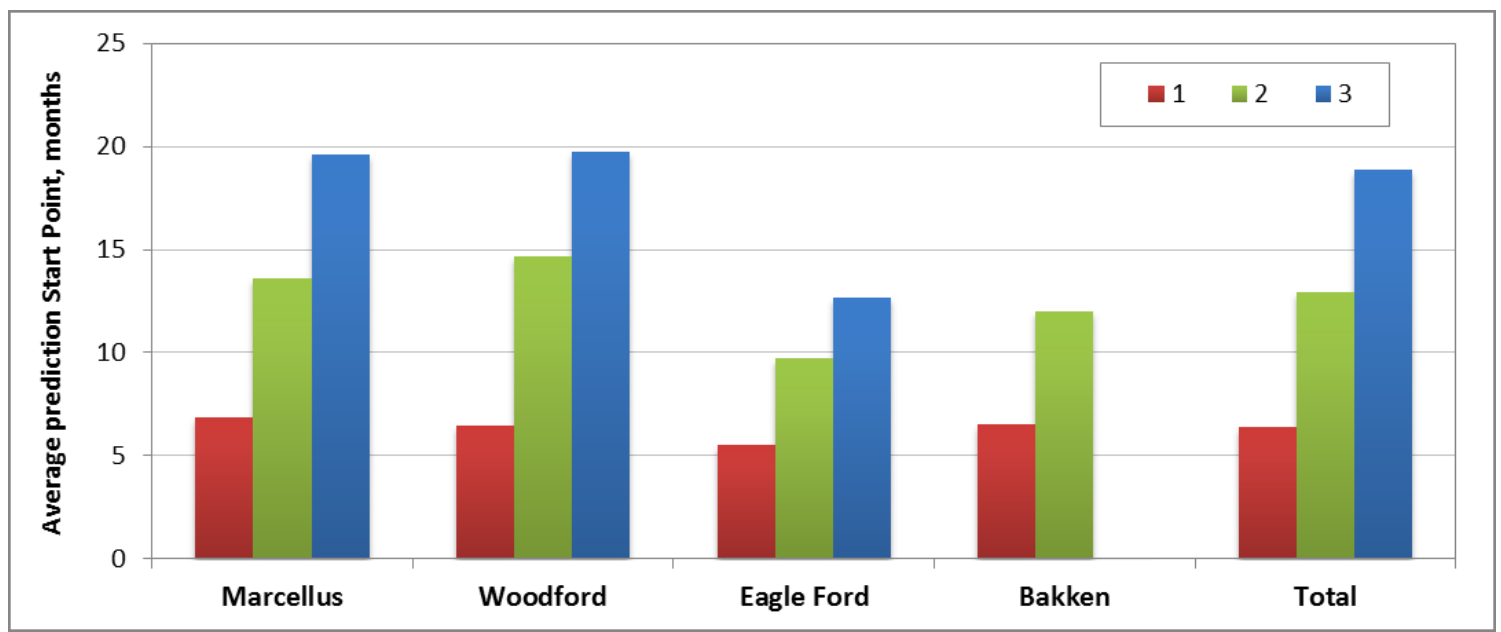

Fig. 72-Average prediction start times for each play.

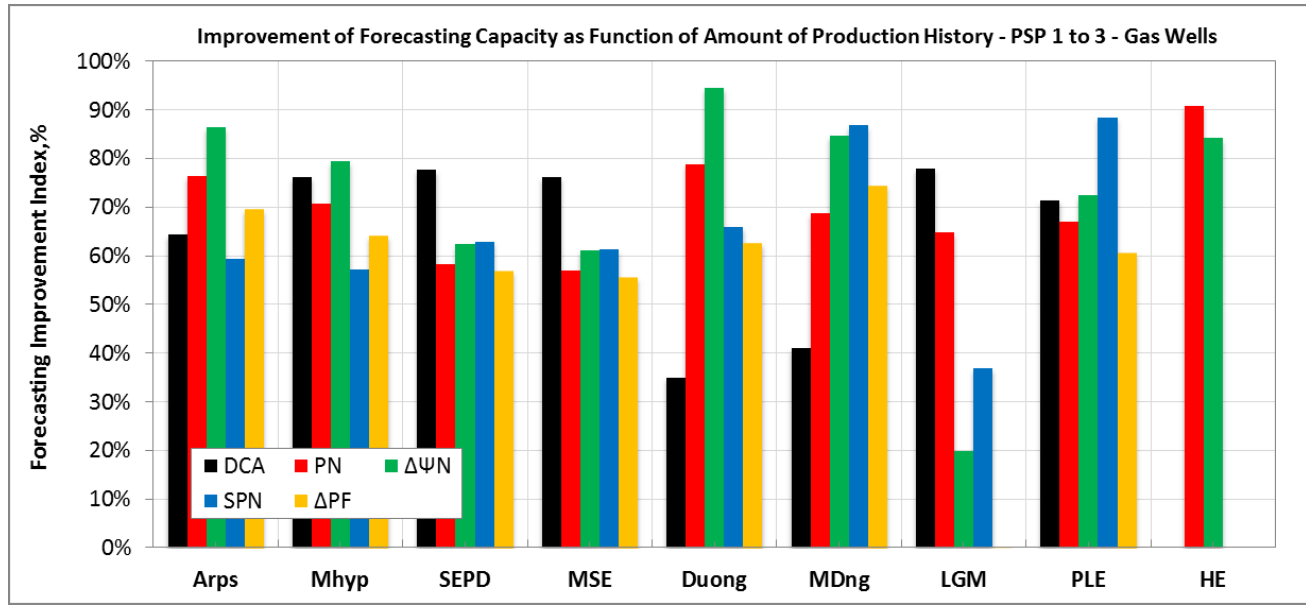

Fig. 73-Forecasting improvement indexes for gas wells (Comparison between PSP 1 and 3).

The evolution of the forecasting accuracy has been measured by a forecasting improvement index (FII), which represents the relative difference between the accuracy of two predictions for the same well (See Eq. 51). As previously stated, most of the wells in this analysis have 2 or 3 predictions (from different start times). Prediction onset times are presented in Table 15. Therefore, this index indicates how the accuracy (A) changes if more production data is available. Fig. $\mathbf{7 3}$ presents the average FII for gas wells. A positive FII means 
that the accuracy of the estimations improves when more production data is available. On the other hand, a negative FII means that the accuracy of the estimations decreases when more production data is available.

$$
F I I=\left(\frac{A_{1}-A_{n}}{A_{1}}\right) * 100 \%
$$

For gas wells, comparisons were performed between the first and the third prediction scenarios. Regarding the oil wells, most of them have a short production history in relation to the gas wells. Thus, it was not possible to perform more than 2 predictions per well. In fact, in some cases, there is only one prediction per well. In addition, given that the average difference between first and second prediction scenarios is relatively short, comparisons cannot be considered very representative. Therefore, meaningful observations cannot be deducted with confidence for the oil wells.

The main observations from gas wells (Fig. 73) are presented below:

- For most of the DCA models, the $\triangle \Psi N$ technique had a higher improvement capacity than the PN technique, as expected.

- The Duong and Modified Duong were highly benefited from the implementation of Pressure Normalization techniques. Arps' models also presented significant improvements when Pressure Normalization techniques were implemented.

- The traditional DCA technique showed higher improvement capacity than Pressure Normalization techniques for SEPD, MSE and LGM models.

- Modified Hyperbolic and PLE presented intermediate behaviors.

- HE showed to have a high capacity to improve its forecasting potential as more historic data is available. 


\subsubsection{High Pressure Rate Controlled Wells}

Pressure Normalizes techniques have an important application in high pressure, rate controlled wells, mentioned in Section 4.3.2. Given that high deliverability wells are constrained and production rates are maintained at constant values for some time, traditional DCA models cannot be used to forecast production performance. In those cases, pressure-normalized DCA techniques may provide a solution. Fig. $\mathbf{7 4}$ and Fig. 75 present two high pressure Eagle Ford wells that have been rate restricted during 6 month approx. As there is not declination, the DCA models cannot be applied. A simple solution is to skip the inconsistent production history. But in some cases, it could be a big portion of the available information. However, if production rates are corrected by pressure normalization (PNR), a smooth decline trend appears and the entire production history can be included in the forecasting analysis.

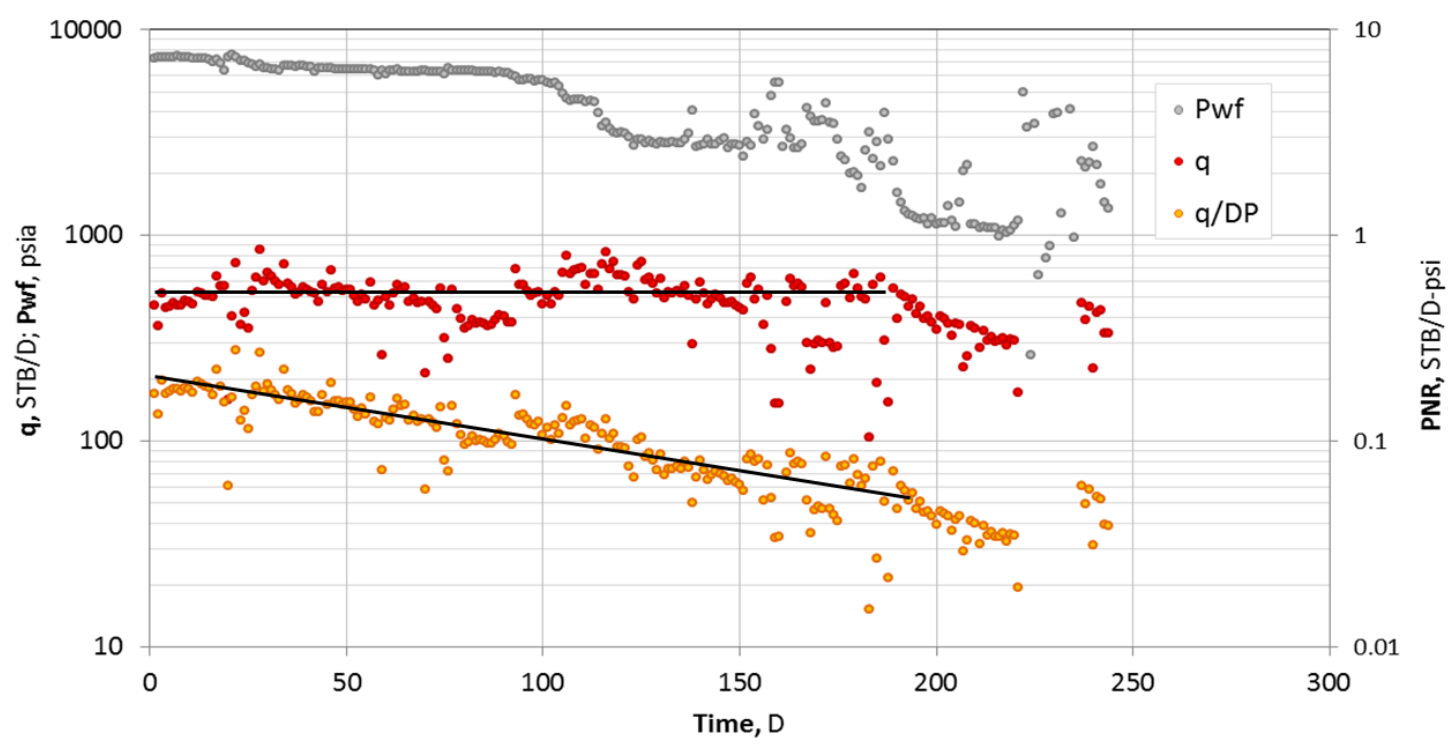

Fig. 74-Application of pressure-normalized rates in well EF-10. Data is presented in a daily basis format.

In this study, 7 out of 16 Eagle Ford wells were classified as high pressure, rate restricted wells, and in 6 out of those 7 wells, PNR enabled the application of decline curve 
analysis on early production data. This is an important advantage, especially for those wells with short production history.

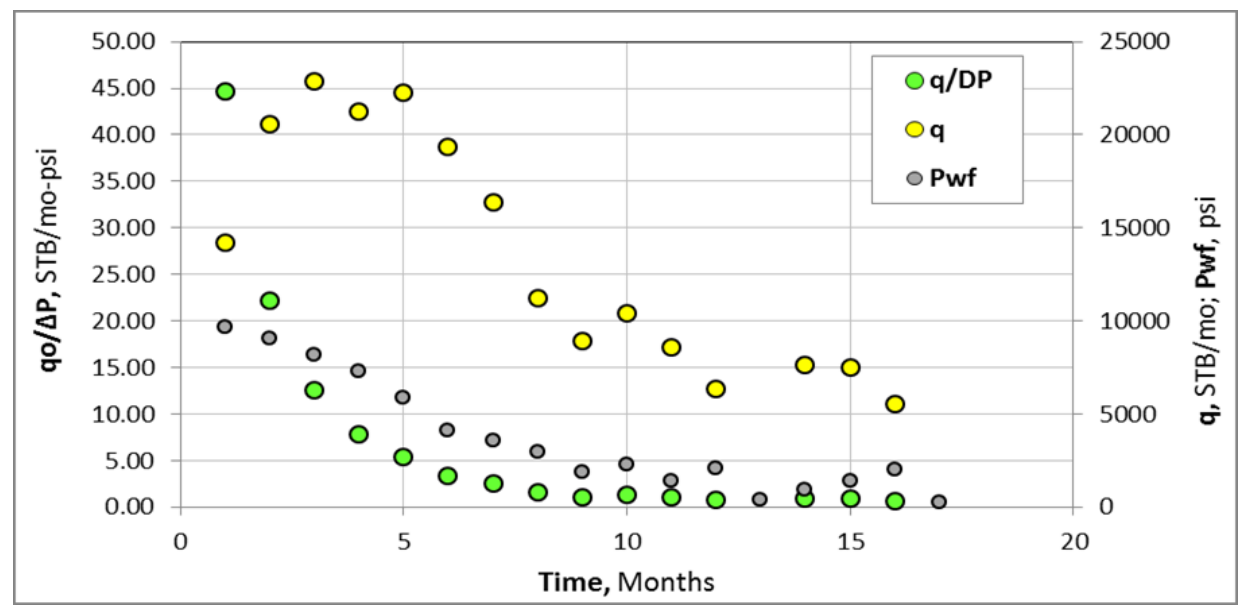

Fig. 75-Application of pressure normalizes rates in well EF-7. Data is presented in a monthly basis format.

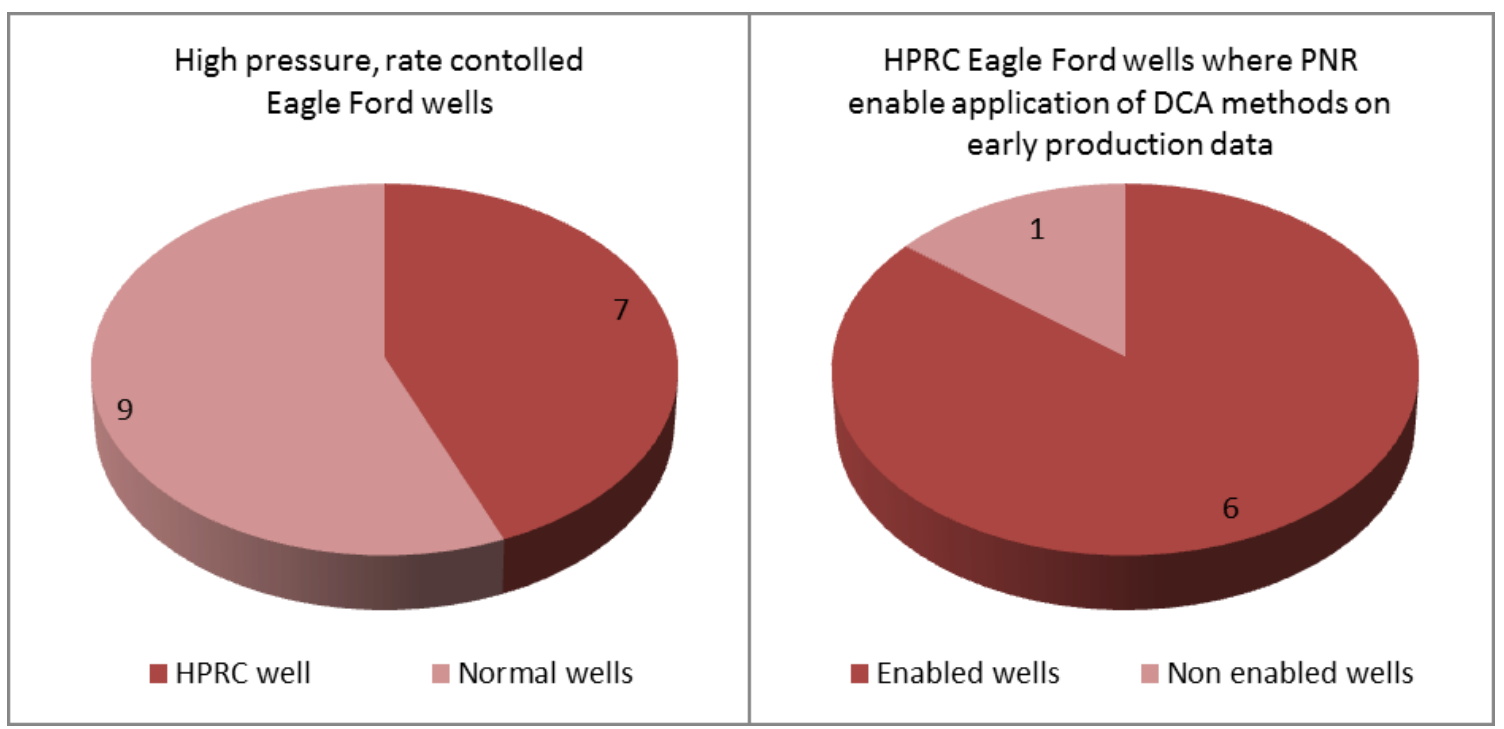

Fig. 76-Number of high pressure, rate controlled Eagle Ford wells identified in this study. 


\section{CONCLUSIONS AND RECOMMENDATIONS}

\subsection{Conclusions}

Based on the flow regime diagnosis and hindcasting analysis of the 47 wells from 4 different unconventional plays (Marcellus, Woodford, Eagle Ford and Bakken) with an average production history of 27.8 months (Minimum: 9; Maximum: 44), the following general conclusions have been deducted from this study:

- It was demonstrated that decline curve analysis based on pressure-normalized rates can be used as a reliable production forecasting technique suited for the interpretation of unconventional wells flowing under unstable operating conditions.

- Pressure normalized DCA (PN-DCA) techniques may offer special advantages over the traditional DCA technique in some specific cases.

- PN-DCA techniques proved to have the special ability to dissociate estimations of future production performance from the existing operating constraints under which production history is developed.

- Pressure normalized rates (PNR) proved to have the ability to offer an alternate solution to high pressure, rate restricted wells by enabling and improving the application of decline curve analysis on early production data.

- Duong and Modified Duong produced outstanding results when applied with the PN-DCA techniques.

- SEPD and MSE were the models that produced the most conservative estimations.

- Overall, the traditional DCA technique provided accurate predictions when the outputs from all DCA models were averaged-a combined models approach. Likewise, Modified Duong's model produced the best solution when the outputs from all techniques were averaged-a combined techniques approach. 
- In general, all the techniques produced conservative estimations. However, the least conservative approaches were provided by the traditional DCA.

- In general, the harmonic decline model generated very accurate and precise estimations-comparable or in some cases even better than the traditional DCA models and techniques.

- In the short term, dual models (Modified Hyperbolic, MSE, and Modified Duong), produced slightly better results in comparison with their parent models (Arps, SEPD and Duong). However, this difference may increase or change in the long term.

Fluid type showed to be a major driver of the behavior of DCA models and techniques. When wells were grouped according to these conditions, they exhibited similar trends of percent error (accuracy). Therefore, additional conclusions are presented below depending on each scenario.

\section{Gas wells scenario:}

- In general, the PN-DCA techniques tend to generate more conservative estimations than the traditional DCA technique.

- The traditional DCA technique provided better results for Arps, Modified Hyperbolic, SEPD and MSE models.

- The forecasting capacity of Duong and Modified Duong significantly improves if applied with the PN-DCA techniques-specifically with SPN and $\triangle P F$.

- The pseudopressure normalization technique $(\Delta \Psi N)$ provided better outputs than the simple pressure normalization technique (PN), which proves that more representative results may be obtained if the pressure-dependent gas properties are included in production analysis by using pseudovariables (pseudopressures, pseudotime, and material balance pseudotime).

- In general, the majority of DCA models improved their forecasting capacity as more historic production was available. Arps, Harmonic Decline, Duong, and 
Modified Duong models presented significant improvements when applied with the pressure normalization techniques, while SEPD and MSE did the same when applied with the traditional DCA technique.

\section{Oil wells scenario:}

- Overall, the PN-DCA techniques provided much better results than the traditional DCA method.

- Modified Hyperbolic and Arps' models yielded better estimations when applied with the pressure normalization (PN) technique, while all the other models worked better with the square pressure normalization (SPN) technique.

- In general, Arps, SEPD, MSE and PLE showed a tendency to underestimate forecasts (generate conservative solutions), while the other models showed a more optimistic behavior.

Regarding the flow regime diagnosis, the following conclusions were deducted:

- More consistent and representative flow regime interpretations may be obtained as diagnostic plots are improved by including the material balance time function, pseudovariables (for gas wells), and pressure-normalized rates. This means that misinterpretations may occur if diagnostic plots are not applied correctly.

- The multi-methods approach proved to be a consistent and reliable solution to identify flow regimes. In addition, uncertainties associated to particular diagnostic plots may be reduced.

- Two side effects were observed when using pressure-normalized rates in flow regime diagnostic plots. The linear flow signature weakened, while transition signatures got stronger.

- Using the material balance time function (MBT) in flow regime diagnostic plots may lead to misinterpretation if production data is scattered, because it tends 
to transform the data in such a way that a fake boundary-dominated flow signature may appear.

\subsection{Recommendations}

The most important restriction of this work is the amount and quality of the input data used in the analysis. Presently, many unconventional plays are being exploited in North America. Each one of these plays has very different fluids and reservoir properties-in addition to local variations. Therefore, a wide range of production performance behaviors may be expected for each well. Moreover, hundreds of wells are usually drilled in each of these plays, which are expected to be on production during long periods of time (tens of years). Considering these observations, the number of wells used in this work is a very small sample compared to the number of existing unconventional wells and all the possible production outcomes. Additionally, the historic production data of these wells is relatively short in comparison to the average expected time of production of an unconventional well. For this reason, some the conclusions of this work-especially those about particular DCA models-may not be valid for long term production forecasts.

Considering these limitations, the following recommendations are proposed:

- A similar study with a larger amount of wells, including cases from different plays with longer historic production data, should be performed to validate the findings of this work.

- A complementary simulation study should be also developed to better understand the long-term behavior of the DCA models and techniques.

- Unconventional liquid-rich plays are becoming more important. Therefore, it is recommended to continue evaluating PN-DCA techniques with more data from these plays. Only $36 \%$ of wells used in this study are oil producers (some of them with short production history and poor quality data). 
Finally, it is unlikely that a single DCA model or technique can provide reliable and accurate solutions for different cases and scenarios. An alternate approach that need to be addressed are the combined solutions-average outputs from different DCA models and/or techniques. Therefore is recommended to develop further studies to assess the applicability and accuracy of this alternate approach. 


\section{REFERENCES}

Abramowitz, M. and Stegun, I. 1964. Handbook of Mathematical Functions, with Formulas, Graphs and Tables. Applied Mathematics. Washington: National Bureau of Standards.

Aderemi, S. and Akpara, K. 2008. Effective Use of Production Surveillance Tool in Forecasting Future Production. Paper presented at the Nigeria Annual International Conference and Exhibition, Abuja, Nigeria. Society of Petroleum Engineers SPE-119732-MS.

Agarwal, R.G., Gardner, D.C., Kleinsteiber, S.W. et al. 1999. Analyzing Well Production Data Using Combined-Type-Curve and Decline-Curve Analysis Concepts. SPE Reservoir Evaluation \& Engineering 2 (5): 478-486. 00057916.

Agnia, A., Alkouh, A.B., and Wattenbarger, R. 2012. Bias in Rate-Transient Analysis Methods: Shale Gas Wells. Paper presented at the SPE Annual Technical Conference and Exhibition, San Antonio, Texas, USA. Society of Petroleum Engineers SPE-159710-MS.

Ahmed, T. 2010. Reservoir Engineering Handbook (4th Edition). In. Burlington, MA. USA: Gulf Professional Publishing (Elsevier). 978-1-85617-803-7

Anderson, D.M., Liang, P., and Mangha, V.O. 2012. Probabilistic Forecasting of Unconventional Resources Using Rate Transient Analysis: Case Studies. Paper presented at the SPE Americas Unconventional Resources Conference, Pittsburgh, Pennsylvania USA. Society of Petroleum Engineers SPE-155737-MS.

Anderson, D.M. and Mattar, L. 2003. Material-Balance-Time During Linear and Radial Flow. Paper presented at the Canadian International Petroleum Conference, Calgary, Alberta. Petroleum Society of Canada PETSOC-2003-201.

Anderson, D.M., Mattar, L., and Rushing, J.A. 2006. Production Data Analysis--Challenges, Pitfalls, Diagnostics. Paper presented at the SPE Annual Technical Conference and Exhibition, San Antonio, Texas, USA. Society of Petroleum Engineers SPE-102048-MS.

Anderson, D.M., Nobakht, M., Moghadam, S. et al. 2010. Analysis of Production Data from Fractured Shale Gas Wells. Paper presented at the SPE Unconventional Gas Conference, Pittsburgh, Pennsylvania, USA. Society of Petroleum Engineers SPE-131787-MS. 
Anderson, S., Anderson, D.M., Epp, K.L. et al. 2012. Pressure Normalized Decline Curve Analysis for Rate-Controlled Wells. Paper presented at the SPE Hydrocarbon Economics and Evaluation Symposium, Calgary, Alberta, Canada. Society of Petroleum Engineers SPE162923-MS.

Arps, J.J. 1945. Analysis of Decline Curves. Trans., AIME, 160: 228-247.

Blasingame, T.A., McCray, T.L., and Lee, W.J. 1991. Decline Curve Analysis for Variable Pressure Drop/Variable Flowrate Systems. Paper presented at the SPE Gas Technology Symposium, Houston, Texas. 1991 Copyright 1991, Society of Petroleum Engineers, Inc. 00021513.

Chen, H.-Y. and Teufel, L.W. 2000. A New Rate-Time Type Curve for Analysis of Tight-Gas Linear and Radial Flows. Paper presented at the SPE Annual Technical Conference and Exhibition, Dallas, Texas. Copyright 2000, Society of Petroleum Engineers Inc. 00063094.

Clark, A.J., Lake, L.W., and Patzek, T.W. 2011. Production Forecasting with Logistic Growth Models. Paper presented at the SPE Annual Technical Conference and Exhibition, Denver, Colorado, USA. Society of Petroleum Engineers SPE-144790-MS.

Clarkson, C.R. 2013a. Production Data Analysis of Unconventional Gas Wells: Review of Theory and Best Practices. International Journal of Coal Geology 109-110 (0): 101-146.

Clarkson, C.R. 2013b. Production Data Analysis of Unconventional Gas Wells: Workflow. International Journal of Coal Geology 109-110 (0): 147-157.

Clarkson, C.R. and Pedersen, P.K. 2010. Tight Oil Production Analysis: Adaptation of Existing Rate-Transient Analysis Techniques. Paper presented at the Canadian Unconventional Resources and International Petroleum Conference, Calgary, Alberta, Canada. Society of Petroleum Engineers SPE-137352-MS.

Duong, A.N. 2010. An Unconventional Rate Decline Approach for Tight and Fracture-Dominated Gas Wells. Paper presented at the Canadian Unconventional Resources and International Petroleum Conference, Calgary, Alberta, Canada. Society of Petroleum Engineers SPE-137748-MS.

El-Banbi, A., H. and Wattenbarger, R.A. 1998. Analysis of Linear Flow in Gas Well Production. Paper presented at the SPE Gas Technology Symposium, Calgary, Alberta, Canada. 1998 Copyright 1998, Society of Petroleum Engineers Inc. 00039972. 
Fekete. Rate Transient Analysis Poster. 2013. http://www.fekete.com/SiteCollectionDocuments/Posters/rta-poster.pdf.

Fekete. Theory and Equations. 2013. http://www.fekete.com/SAN/TheoryAndEquations/HarmonyTheoryEquations/default. htm.

Fetkovich, M.J. 1980. Decline Curve Analysis Using Type Curves. SPE Journal of Petroleum Technology 32 (6): 1065-1077. SPE-4629.

Fetkovich, M.J., Fetkovich, E.J., and Fetkovich, M.D. 1996. Useful Concepts for Decline Curve Forecasting, Reserve Estimation, and Analysis. SPE Reservoir Engineering 11 (1): 13-22. SPE-28628.

Fetkovich, M.J., Vienot, M.E., Bradley, M.D. et al. 1987. Decline Curve Analysis Using Type Curves: Case Histories. SPE Formation Evaluation 2 (4): 637-656. SPE-13169.

Freeborn, R. and Russell, B. 2012. How to Apply Stretched Exponential Equations to Reserve Evaluation. Paper presented at the SPE Hydrocarbon Economics and Evaluation Symposium, Calgary, Alberta, Canada. Society of Petroleum Engineers SPE-162631-MS.

Gale, J.F.W. and Holder, J. 2010. Natural Fractures in Some Us Shales and Their Importance for Gas Production. Geological Society, London, Petroleum Geology Conference Series 7: 1131-1140.

Ilk, D., Jenkins, C.D., and Blasingame, T.A. 2011. Production Analysis in Unconventional Reservoirs - Diagnostics, Challenges, and Methodologies. Paper presented at the North American Unconventional Gas Conference and Exhibition, The Woodlands, Texas, USA. Society of Petroleum Engineers SPE-144376-MS.

Ilk, D., Rushing, J.A., Perego, A.D. et al. 2008. Exponential Vs. Hyperbolic Decline in Tight Gas Sands - Understanding the Origin and Implications for Reserve Estimates Using Arps' Decline Curves. Paper presented at the SPE Annual Technical Conference and Exhibition, Denver, Colorado, USA. Society of Petroleum Engineers SPE-116731-MS.

Joshi, K. and Lee, W. 2013. Comparison of Various Deterministic Forecasting Techniques in Shale Gas Reservoirs. Paper presented at the 2013 SPE Hydraulic Fracturing Technology Conference, The Woodlands, TX, USA. Society of Petroleum Engineers SPE-163870-MS.

Kanfar, M. and Wattenbarger, R. 2012. Comparison of Empirical Decline Curve Methods for Shale Wells. Paper presented at the SPE Canadian Unconventional Resources Conference, Calgary, Alberta, Canada. Society of Petroleum Engineers SPE-162648-MS. 
Liang, P., Thompson, J.M., and Mattar, L. 2012. Importance of the Transition Period to Compound Linear Flow in Unconventional Reservoirs. Paper presented at the SPE Canadian Unconventional Resources Conference, Calgary, Alberta, Canada. Society of Petroleum Engineers SPE-162646-MS.

Luo, S., Neal, L., Arulampalam, P. et al. 2010. Flow Regime Analysis of Multi-Stage HydraulicallyFractured Horizontal Wells with Reciprocal Rate Derivative Function: Bakken Case Study. Paper presented at the Canadian Unconventional Resources and International Petroleum Conference, Calgary, Alberta, Canada. Society of Petroleum Engineers SPE137514-MS.

Mangha, V.O., Ilk, D., Blasingame, T.A. et al. 2012. Practical Considerations for Decline Curve Analysis in Unconventional Reservoirs --- Application of Recently Developed Rate-Time Relations. Paper presented at the SPE Hydrocarbon Economics and Evaluation Symposium, Calgary, Alberta, Canada. Society of Petroleum Engineers SPE-162910-MS.

Mattar, L. and Anderson, D. 2005. Dynamic Material Balance(Oil or Gas-in-Place without ShutIns). Paper presented at the Canadian International Petroleum Conference, Calgary, Alberta. Petroleum Society of Canada PETSOC-2005-113.

Mattar, L. and Anderson, D.M. 2003. A Systematic and Comprehensive Methodology for Advanced Analysis of Production Data. Paper presented at the SPE Annual Technical Conference and Exhibition, Denver, Colorado. Society of Petroleum Engineers SPE84472.

Mattar, L. and McNeil, R. 1995. The "Flowing" Material Balance Procedure. Paper presented at the Annual Technical Meeting, Calgary, Alberta. Petroleum Society of Canada PETSOC95-77.

Mishra, S. 2012. A New Approach to Reserves Estimation in Shale Gas Reservoirs Using Multiple Decline Curve Analysis Models. Paper presented at the SPE Eastern Regional Meeting, Lexington, Kentucky, USA. Society of Petroleum Engineers SPE-161092-MS.

Nobakht, M. and Clarkson, C.R. 2012a. Multiwell Analysis of Multifractured Horizontal Wells in Tight/Shale Gas Reservoirs. Paper presented at the SPE Canadian Unconventional Resources Conference, Calgary, Alberta, Canada. Society of Petroleum Engineers SPE162831-MS.

Nobakht, M. and Clarkson, C.R. 2012b. A New Analytical Method for Analyzing Linear Flow in Tight/Shale Gas Reservoirs: Constant-Rate Boundary Condition. SPE Reservoir Evaluation \& Engineering 15 (1): pp. 51-59. SPE-143990-PA. 
Nobakht, M. and Mattar, L. 2009. Diagnostics of Data Quality for Analysis of Production Data. Paper presented at the Canadian International Petroleum Conference, Calgary, Alberta. Petroleum Society of Canada PETSOC-2009-137.

Nobakht, M., Mattar, L., Moghadam, S. et al. 2010. Simplified yet Rigorous Forecasting of Tight/Shale Gas Production in Linear Flow. Paper presented at the SPE Western Regional Meeting, Anaheim, California, USA. Society of Petroleum Engineers SPE133615-MS.

Rasdi, M.F. and Chu, L. 2012. Diagnosing Fracture Network Pattern and Flow Regime Aids Production Performance Analysis in Unconventional Oil Reservoirs. Paper presented at the SPE/EAGE European Unconventional Resources Conference and Exhibition, Vienna, Austria. Society of Petroleum Engineers SPE-151623-MS.

Thompson, J.M., Mangha, V.O., and Anderson, D.M. 2011. Improved Shale Gas Production Forecasting Using a Simplified Analytical Method-a Marcellus Case Study. Paper presented at the North American Unconventional Gas Conference and Exhibition, The Woodlands, Texas, USA. Society of Petroleum Engineers SPE-144436-MS.

Valko, P.P. and Lee, W.J. 2010. A Better Way to Forecast Production from Unconventional Gas Wells. Paper presented at the SPE Annual Technical Conference and Exhibition, Florence, Italy. Society of Petroleum Engineers SPE-134231-MS.

Wattenbarger, R.A., El-Banbi, A.H., Villegas, M.E. et al. 1998. Production Analysis of Linear Flow into Fractured Tight Gas Wells. Paper presented at the SPE Rocky Mountain Regional/Low-Permeability Reservoirs Symposium, Denver, Colorado. Society of Petroleum, Engineers 00039931. 